\title{
Extraction of Active and Sterile Neutrino Mixing Parameters with the Sudbury Neutrino Observatory
}

by

Gordana Tešić

\author{
A Dissertation submitted to \\ the Faculty of Graduate Studies and Research \\ in partial fulfilment of \\ the requirements for the degree of \\ Doctor of Philosophy \\ Ottawa-Carleton Institute for Physics, \\ Department of Physics, Carleton University, \\ Ottawa, Ontario, Canada \\ December, 2008
}

Copyright (C)Gordana Tešić

2008 


$\begin{array}{ll}\begin{array}{l}\text { Library and } \\ \text { Archives Canada }\end{array} & \begin{array}{l}\text { Bibliothèque et } \\ \text { Archives Canada }\end{array} \\ \begin{array}{l}\text { Published Heritage } \\ \text { Branch }\end{array} & \begin{array}{l}\text { Direction du } \\ \text { Patrimoine de l'édition }\end{array} \\ \begin{array}{l}\text { 395 Wellington Street } \\ \text { Ottawa ON K1A 0N4 } \\ \text { Canada }\end{array} & \begin{array}{l}\text { 395, rue Wellington } \\ \text { Ottawa ON K1A 0N4 } \\ \text { Canada }\end{array}\end{array}$

Your file Votre référence ISBN: 978-0-494-47493-8

Our file Notre référence

ISBN: 978-0-494-47493-8

NOTICE:

The author has granted a nonexclusive license allowing Library and Archives Canada to reproduce, publish, archive, preserve, conserve, communicate to the public by telecommunication or on the Internet, loan, distribute and sell theses worldwide, for commercial or noncommercial purposes, in microform, paper, electronic and/or any other formats.

The author retains copyright ownership and moral rights in this thesis. Neither the thesis nor substantial extracts from it may be printed or otherwise reproduced without the author's permission.
AVIS:

L'auteur a accordé une licence non exclusive permettant à la Bibliothèque et Archives Canada de reproduire, publier, archiver, sauvegarder, conserver, transmettre au public par télécommunication ou par l'Internet, prêter, distribuer et vendre des thèses partout dans le monde, à des fins commerciales ou autres, sur support microforme, papier, électronique et/ou autres formats.

L'auteur conserve la propriété du droit d'auteur et des droits moraux qui protège cette thèse. $\mathrm{Ni}$ la thèse ni des extraits substantiels de celle-ci ne doivent être imprimés ou autrement reproduits sans son autorisation.
In compliance with the Canadian Privacy Act some supporting forms may have been removed from this thesis.

While these forms may be included in the document page count, their removal does not represent any loss of content from the thesis.
Conformément à la loi canadienne sur la protection de la vie privée, quelques formulaires secondaires ont été enlevés de cette thèse.

Bien que ces formulaires aient inclus dans la pagination, il n'y aura aucun contenu manquant.

\section{Canada}




\section{Abstract}

The Sudbury Neutrino Observatory (SNO) is a 1 kilotonne heavy-water Cerenkov detector designed to study fundamental properties of neutrinos produced by thermonuclear fusion reactions in the core of the Sun. The uniqueness of SNO resides in its capability to distinctively measure the total flux of all active neutrino flavours as well as the flux of electron neutrinos, through the Neutral-Current (NC) and Charged-Current (CC) interactions of neutrinos on deuterium, respectively. The measurements of the $\mathrm{NC}$ and $\mathrm{CC}$ fluxes for neutrinos originated from ${ }^{8} \mathrm{~B}$ disintegration inside the Sun unambiguously proved that neutrinos change their flavour while traveling to the Earth. These results are consistent with predictions from a neutrino oscillation hypothesis on neutrino flavour transitions due to the mixing of massive neutrino states. The NC measurement from SNO also solved the long-standing Solar Neutrino Problem (SNP).

In this dissertation, the measurements of the fundamental properties of neutrinos, in particular their mixing parameters, are presented. Data samples from SNO and other experiments are used to extract the mixing parameters of active and sterile neutrino states. Under the assumption on the two-neutrino oscillation hypothesis, the mixing parameters for active neutrinos (the squared-mass difference $\Delta m^{2}$ and the mixing angle $\theta$ ) are obtained from a global analysis of solar 
and reactor neutrino data. The extracted mixing parameters from this analysis are $\Delta m^{2}=7.59_{-0.19}^{+0.21} \times 10^{-5} \mathrm{eV}^{2}$ and $\theta=34.4_{-1.2}^{+1.3}$ degrees. The errors on both parameters are reduced compared to the previous results from SNO, that further constrains the solar neutrino mixing parameter region. The mixing parameters for the sterile neutrino state (the ratio $R_{\Delta}=\Delta m_{01}^{2} / \Delta m_{21}^{2}$ and the mixing angle $\sin ^{2} 2 \alpha$ ) are determined by comparing the predictions from a weakly mixed sterile neutrino model with the solar neutrino data. For the first time a complete parameter region for the weakly mixed sterile state is fully scanned numerically to place the error on $R_{\Delta}$ and to set an upper limit at $90 \%$ CL on $\sin ^{2} 2 \alpha$. A global solar neutrino analysis yields $R_{\Delta}=0.11_{-0.03}^{+0.04}$ and places an upper limit of $\sin ^{2} 2 \alpha<9.9 \times 10^{-3}$ at $90 \% \mathrm{CL}$. This result shows that the rare effects from physics beyond the three active neutrino scenario cannot be excluded, yet.

Future prospects and challenge in solar neutrino physics are also summarized. 


\section{Statement of Originality}

The analyses presented in this dissertation would not be possible without joint

effort of many analysts of the SNO collaboration that assure the best quality of data taking and selection, detector calibration, estimation of the systematic errors and signal extraction; and also contribute to hardware maintenance and software development. The author contributed to shift and data taking in Sudbury and was a member of the run selection committee.

This dissertation presents the analyses and results obtained by the author, except for cases mentioned in the text or listed bellow.

SNO analyses software packages SNOMAN and QSNO have been used by the author for the detector's live-time calculation and physics interpretation. A specific module of QSNO software, called QPHYSICS was the main tool used in the extraction of the solar neutrino mixing parameters. QPHYSICS was originally written by Fraser Duncan and Mark Chen. The valuable improvements to this module were brought by Yasuo Takeuchi, including the computation of the energy related spectral derivatives at each point on the $\tan ^{2} \theta-\Delta m^{2}$ plane, and algorithms for the computation of the model predictions for many solar neutrino experiments. The author maintained and further developed QPHYSICS module for the purposes of this dissertation (e.g. $\chi^{2}$ and EML methods). 
The signal extraction provided us with the data samples and associated statistical correlations for SNO. The systematic errors for these data were obtained from the energy and optics, data cleaning and neutron working groups.

The two-neutrino analyses before the third phase of SNO data taking have been performed by the author under the leading role of Mark Chen, who also upgraded the software to handle data set from the SNO second phase of running. The characteristics of the Borexino detector were coded by José Maneira. The most recent solar model data files were created by Olivier Simard.

For the sterile neutrino analyses, the sampling of the neutrino production region inside the Sun has been chosen to match the one of Pedro de Holanda and Alexei Smirnov. The full numerical implementation of the method and data analyses have been done by the author of this dissertation. 


\section{Acknowledgements}

Firstly, I would like to express my gratitude to my supervisor Alain Bellerive for his support and encouragement during my graduate studies at Carleton University. I would like to thank him for being an exceptionally great advisor, for being available at all times to discuss not only my research, but also many other important matters that went beyond the direct scope of my studies. Regardless of the nature of my questions and concerns, he always provided me with excellent advice and immediate support. I thank him for inspiring me with all his optimism, great energy, and creativity, resulting in many excellent research ideas that, combined with a freedom given to his student, resulted in my graduate studies being a less stressful and more enjoyable experience. Finally, I would like to thank him for constantly reminding me that doing physics should be fun, as well as that life has many aspects that must not be neglected regardless of a graduate student's preoccupation with courses and research.

I would like also to thank the faculty members at the SNO group at Carleton. I am very thankful to Richard Hemingway for his great spirit that has cheered me up for several years, for his invaluable sense of clear and sharp thoughts in any possible situation. I thank him for his serious and always helpful advice, for his concerns on not only my research, but also many other aspects of my graduate 
life. I thank him for the technical support that he kindly provided to me, which made my graduate student's life much easier, and, finally, for his visits to my long and lonely evening shifts in Sudbury, bringing me fresh food and warm coffee that combined with his great sense of humor always raised up my energy. I wish to thank Peter Watson for providing me with numerous excellent ideas during my studies of the three-neutrino systems, and for being so kind to lend me his Mac, without which the writing of this dissertation would be inexplainable more difficult. Finally, thanks to him for his willingness to teach me cosmology, which I planned to study for many years. I thank David Sinclair for all intelligible discussions, his kindness and for the warm and unforgettable Christmas with his family during my first year at Carleton. I wish to thank Cliff Hargrove for his clever comments during our weekly Carleton SNO meeting and for his great empathy that resulted in my first laptop for the purpose of research to be borrowed from him.

Many thanks to my colleagues and office-mates: Pierre-Luc Drouin for his significant help in dealing with a number of obstacles during compilations of my code, great discussions and the best music during long trips to Sudbury; Olivier Simard for making available the most recent solar model data files, help with $\mathrm{C}++$ programming, for his ability to always make a great joke at the right time and bring everybody's spirit up, and for teaching me how to safely drive a car under a terrible winter storm; Etienne Rollin for showing me many ROOT and Unix tips, teaching me how to play baseball, a great sense of humor and all the nice time that we spent working together. I wish to thank former and present research associates: David Waller for his willingness to help anytime, for his clever comments and for his best effort to show me as much as possible of interesting sites in London during 
a single day; Feng Zeng for always been ready to help with the transfer of the large amount of data files that I needed during my live-time calculation, for being a great office-mate and, finally, for educating me on many varieties of green-tea including their preparation; and Khalil Boudjemline for his interest in my research and much advice regarding my time management. Also many thanks to the entire Physics Department at Carleton, faculty, administrators, IT support, research associates and many other students for not only being helpful and kind, but for creating a very enjoyable atmosphere at work as well.

I would also like to express my thanks to the entire SNO Collaboration. It has been an honour and a pleasure to work with my great colleagues from SNO. I own thanks to Mark Chen for being a leader of the Physics Interpretation group in the past, from whom I learned many details on the extraction of the neutrino mixing parameters, for providing us with great suggestions and always intelligible comments; Yasuo Takeuchi for making invaluable improvements to the SNO physics interpretation software and his very helpful "how-to" pages; José Maneira for his work on the model predictions for the Borexino experiment; and Keith Rielage for being a great and always cheerful leader of the live-time working group, and coordinator for the publication of the recent results from SNO.

I must express my gratefulness to Pedro de Holanda and Alexei Smirnov, whose sterile neutrino model was used in this dissertation, for being very kind in providing me with all necessary information regarding their own calculations, which resulted in my code being thoroughly tested and more efficient.

I wish to thank too my close friends and relatives for their love and support, for their understanding, for their creativity that has always inspired me and for challenging my mind in the most stimulating way. Many thanks to my grandfather 
Stevan Savić for long inspiring conversations, unlimited love, support and all transAtlantic airplane tickets! Infinite thanks to Marko Tešić, Ljubiša Tešić, Ivan Grujičič, Vojislav Tešić, Sonia Amiti, Aleksandar Trajković, Veljko Janković and many others.

I would like to thank Deborah Fish for her great and helpful advice.

And finally, I would like to thank my lovable family: my parents Radoslav and Olivera Tešić, sister Danijela Grujičič and brother Milutin Tešić for their infinite love, support, encouragement and for the best advice in the entire (Multi)Universe! Without them, all this work would not be plausible. 


\section{Dedication}

To my family and friends. 


\section{Contents}

1 Introduction 1

2 Physics of Neutrinos $\quad 5$

2.1 Neutrinos in the Standard Model . . . . . . . . . . . . 5

2.2 Neutrino Mass . . . . . . . . . . . . . . . 8

2.3 Neutrino Mixing . . . . . . . . . . . . . . . 10

2.4 Neutrino Oscillation . . . . . . . . . . . . . . 11

2.4.1 Two-Neutrino Approximation ............ 15

2.4.2 Weakly Mixed Sterile Neutrino Model . . . . . . . . . 17

2.5 Matter Effect . . . . . . . . . . . . . . . 19

2.5.1 MSW Effect for the Two-Neutrino Scenario . . . . . . 21

2.5.2 MSW Effect for the Sterile Scenario . . . . . . . . . . 23

2.6 Summary .............................. 25

3 Solar Neutrinos $\quad 26$

3.1 Neutrino Production . . . . . . . . . . . . . 27

3.2 The Standard Solar Model . . . . . . . . . . . . . . . . . . . 31

3.3 Solar Neutrino Problem . . . . . . . . . . . . . . 34

3.4 Finally a Solution . . . . . . . . . . . . . . . 39 
4 Experimental Apparatus $\quad 41$

4.1 The Sudbury Neutrino Observatory . . . . . . . . . . . . . . 42

4.2 Signature of Neutrinos and Interactions . . . . . . . . . . . . . 44

4.2 .1 Čerenkov Radiation . . . . . . . . . . . . . 45

4.2.2 The Charged-Current Interaction . . . . . . . . . . 46

4.2 .3 The Neutral-Current Interaction . . . . . . . . . . . . 47

4.2.4 The Elastic Scattering Interaction . . . . . . . . . . . . 49

4.3 PMT Energy Response . . . . . . . . . . . . . . . . . . 50

$5 \quad$ Data Selection and Livetime $\quad 53$

5.1 Run Selection . . . . . . . . . . . . . . . 54

5.1.1 FPS and SPS checks of runs ............ 54

5.1 .2 Selection of the Final Data Set . . . . . . . . 57

5.2 Livetime . . . . . . . . . . . . . . . . . . . 59

5.2 .1 The Clocks ................... 60

5.2 .2 Raw Livetimes ................. 61

5.2 .3 The Burst Cuts . . . . . . . . . . . . . 64

5.2 .4 The Dead Times . . . . . . . . . . . . 67

5.2 .5 Uncertainties . . . . . . . . . . . . . 68

5.2.6 Livetime as a Function of Zenith Angle . . . . . . . . . . 71

5.3 Results . . . . . . . . . . . . . . . . 72

6 SNO Data Samples $\quad 74$

6.1 Data Samples . . . . . . . . . . . . . . . 74

6.2 Systematic Uncertainties . . . . . . . . . . . . . 83

6.2.1 Neutron Capture Efficiency ............ 83 
6.2 .2 Energy Scale $\ldots \ldots \ldots \ldots \ldots \ldots$

6.2 .3 Energy Resolution . . . . . . . . . . . . . . 85

6.2 .4 Energy Non-Linearity . . . . . . . . . . . . . . . 86

6.2.5 Vertex Resolution and Accuracy . . . . . . . . . . 87

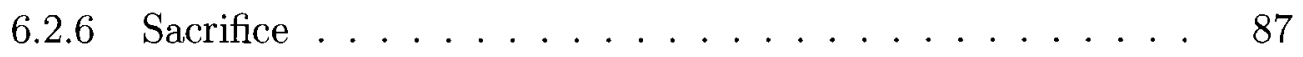

6.2 .7 Event Isotropy $\ldots \ldots \ldots \ldots$

6.2 .8 Angular Resolution . . . . . . . . . . . . . . . . . 88

6.2 .9 Radial Energy Bias . . . . . . . . . . . . . . . 89

6.2.10 Reconstructed Energy Dependance ... . . . . . . 89

6.2 .11 Diurnal Effects . . . . . . . . . . . . . 9 90

6.2 .12 NCD Array . . . . . . . . . . . . . . 90

6.2.13 Correlation between the SNO Systematics . . . . . . . 91

$\begin{array}{lll}7 & \text { Results - Two-Active Neutrino Model } & 100\end{array}$

7.1 Experimental Data . . . . . . . . . . . . . . . . 100

7.2 Two-Neutrino Mixing Model Predictions . . . . . . . . . . . 101

7.2.1 Solar Neutrino Survival Probabilities . . . . . . . . . . 101

7.2.2 The Expected Neutrino Yields . . . . . . . . . . . . . . 103

7.3 Extraction of the Mixing Parameters . . . . . . . . . 108

7.3.1 Covariance $\chi^{2}$ Method . . . . . . . . . . . . . 108

7.3.2 Experimental Systematic Uncertainties . . . . . . . 110

7.3.3 Calculation of the Partial Derivatives $\boldsymbol{\beta}_{\boldsymbol{i} \boldsymbol{k}} \ldots \ldots \ldots \ldots 111$

7.3.4 Theoretical Uncertainties . . . . . . . . . . . . . . . . 123

7.4 The Results . . . . . . . . . . . . . . . . . . . 129

7.4.1 The Results - SNO-only . . . . . . . . . . . . 130 
7.4.2 Global Solar and Solar+KamLAND Results . . . . . . 133

7.5 Summary . . . . . . . . . . . . . . 138

8 Results - the Sterile Neutrino Model $\quad 141$

8.1 Motivation . . . . . . . . . . . . . . . . . 141

8.2 Solar Neutrino Transition Probabilities . . . . . . . . . . . . 145

8.2.1 Neutrino Propagation for the Sun and Vacuum . . . . . 145

8.2 .2 The Earth Effect . . . . . . . . . . . . . . . . 150

8.3 Method ...................... 156

8.4 The Results - SNO Only . . . . . . . . . . . . . . 158

8.5 Solar Global Results . . . . . . . . . . . . . . . . 167

8.5.1 Results - Partial SNO-II . . . . . . . . . . . . . 167

8.5 .2 Results - Full SNO-II . . . . . . . . . . . . . . . 171

8.5.3 Results - After SNO-III . . . . . . . . . . . . 172

8.6 Summary . . . . . . . . . . . . . 180

9 Future Prospects 183

9.1 Low Energy Solar Neutrino Data . . . . . . . . . . . . . . . 183

$9.1 .1 \quad$ LETA Expectation . . . . . . . . . . . . . . . . 183

9.1 .2 Sterile Neutrinos . . . . . . . . . . . . . . . . . 184

9.1.3 Three Active Neutrinos . . . . . . . . . . . . . . . 185

9.2 Maximum Likelihood Method . . . . . . . . . . . . . . . 186

9.3 Discussion . . . . . . . . . . . . . . . . . . . . . . . . . . . . . 193

10 Conclusion $\quad 194$

$\begin{array}{ll}\text { A The Three-Active Neutrinos } & 198\end{array}$ 
B EML Method with Pulls

201

Glossary

204 


\section{List of Tables}

3.1 Neutrino fluxes from BP00 [38], BP04 [39], and BS05(OP) [40] for the principal fusion reactions. . . . . . . . . . . . 34

4.1 Electron energy response parameters for the three phases of SNO.

5.1 Summary of raw livetimes for the NCD data set. . . . . . . . . 64

5.2 Summary of raw livetime, individual cuts, combined deadtime and

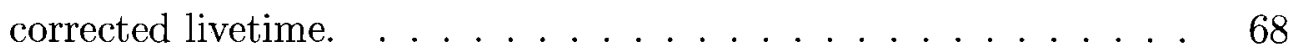

5.3 Comparison of cut times percentages of the raw livetime calculated by LSP and by PGT. . . . . . . . . . . . . . . . . . 69

5.4 Summary of the corrected livetimes from LSP and PGT. . . . . 70

5.5 Summary of day and night corrected livetimes from LSP and PGT. 71

5.6 Summary of final livetimes with their uncertainties for the NCD

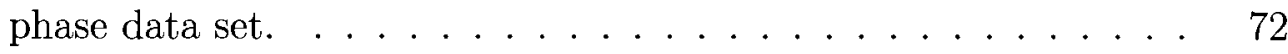

6.1 SNO-I day and night $\mathrm{CC}+\mathrm{NC}+\mathrm{ES}+$ backgrounds summed kinetic

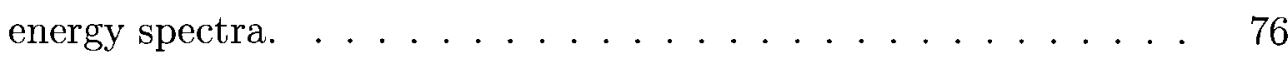

6.2 SNO-I day and night energy spectra for neutron background (NCBG). 77

6.3 SNO-I day and night energy spectra for low energy Čerenkov background $(\mathrm{LEB}) . \ldots \ldots \ldots \ldots \ldots \ldots \ldots \ldots \ldots$ 
6.4 SNO-II CC electron kinetic energy spectra for day and night. . . . 79

6.5 SNO-II ES and NC fluxes from a shape-unconstrained signal extraction for day and night. . . . . . . . . . . . . 80

6.6 SNO-III CC, ES and NC fluxes with their statistical and systematical uncertainties from a shape-unconstrained signal extraction.

6.7 SNO-III statistical correlation coefficients for the CC, ES and NC fluxes. ......................... 80

6.8 SNO-II statistical correlation coefficients for day CC spectrum, ES and $\mathrm{NC}$ fluxes. ..................... 81

6.9 SNO-II statistical correlation coefficients for night CC spectrum, ES and NC fluxes. . . . . . . . . . . . . . . . 82

6.10 Systematic uncertainties for the SNO-I data set. . . . . . . . . . 94

6.11 Systematic uncertainties for the SNO-II data set. . . . . . . . . 95

6.12 Systematic uncertainties for the SNO-III data set. . . . . . . . . 96

6.13 Correlation coefficients for the same sources of systematic uncertainties affecting different types of signals for the SNO-II data set.

6.14 Correlation coefficients for the same sources of systematic uncertainties affecting different types of signals for the SNO-III data set. 97

6.15 Breakdown of the individual components for the systematic uncertainties on energy scale and resolution for the three phases of SNO. 98

6.16 Systematic uncertainties on the neutron efficiency measurement from the calibration data in the pure $\mathrm{D}_{2} \mathrm{O}$ phase. . . . . . . . 98

6.17 Systematic uncertainties on the neutron efficiency measurement from the calibration data in the salt phase. . . . . . . . . 
6.18 Correlation coefficients for the systematic uncertainties partially correlated between the SNO-I, SNO-II and SNO-III phases. . . . . 99

7.1 The number of electron and deuteron targets per unit volume in the SNO detector. . . . . . . . . . . . . . 107

7.2 SNO-I day relative errors $\left(\Delta Y_{i} / Y_{i}=\beta_{i k} \Delta S_{k}\right.$ in percent) computed under assumption of the non-oscillating model. . . . . . . . . . . 118

7.3 SNO-I night relative errors $\left(\Delta Y_{i} / Y_{i}=\beta_{i k} \Delta S_{k}\right.$ in percent $)$ computed under assumption of the non-oscillating model. . . . . . . 119

7.4 Example of day and night SNO-I relative errors $\left(\Delta Y_{i} / Y_{i}=\beta_{i k} \Delta S_{k}\right.$ in percent) for energy scale and energy resolution systematic un-

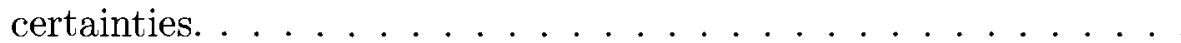

7.5 SNO-II day and night relative errors $\left(\Delta Y_{i} / Y_{i}=\beta_{i k} \Delta S_{k}\right.$ in percent) on CC spectra, which are computed under the non-oscillating

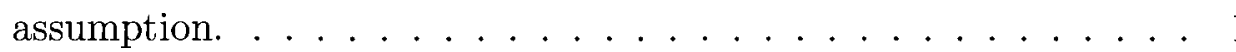

7.6 Relative errors $\left(\Delta Y_{i} / Y_{i}=\beta_{i k} \Delta S_{k}\right.$ in percent) on NC and ES day and night fluxes for SNO-II. . . . . . . . . . . . . . . 122

7.7 Relative errors $\left(\Delta Y_{i} / Y_{i}=\beta_{i k} \Delta S_{k}\right.$ in percent) on CC, ES and NC fluxes for SNO-III phase. . . . . . . . . . . . . . . 123

7.8 The errors on the cross-section $\Delta \ln C_{i k}$ for the chlorine ( $1 \sigma$ values) and gallium ( $3 \sigma$ values) experiments. . . . . . . . . 125

7.9 Fractional $1 \sigma$ uncertainties $\Delta \ln X_{k}$ associated with the astrophysical parameters $X_{k} \ldots \ldots \ldots \ldots \ldots$

7.10 Fractional $1 \sigma$ uncertainties $\Delta \ln X_{l k}$ for the decomposed metallicity parameters $Z_{l} / X \ldots \ldots \ldots \ldots \ldots \ldots$ 
7.11 Logarithmic partial derivatives on neutrino fluxes $\alpha_{i k}=\partial \ln \phi_{i} / \partial \ln X_{k} .128$

7.12 Logarithmic partial derivatives on neutrino fluxes $\alpha_{i k}=\partial \ln \phi_{i} / \partial \ln X_{k}$ with respect to the individual metallicity uncertainties. . . . . . 128

7.13 SNO-only neutrino oscillation best-fit parameters. . . . . . . . . 134

7.14 Global solar only and global solar+KamLAND best-fit parameters. 140

8.1 Upper limits on the sterile neutrino mixing parameters at 90\% CL from the SNO-I+SNO-II (partial) oscillation analyses. . . . . . . 159

8.2 Upper limits on the sterile neutrino mixing parameters at 90\% CL from the SNO-only (SNO-I+SNO-II+SNO-III) oscillation analyses. 163

8.3 Global solar best-fit parameters with the partial and full data samples from the SNO-II phase. . . . . . . . . . . . . . . . . . . 171

8.4 Global solar best-fit parameters after the SNO-III phase. . . . . 176

9.1 SNO-I neutrino oscillation best-fit parameters. . . . . . . . . . . 189 


\section{List of Figures}

3.1 Energy spectra of neutrino fluxes from the $p p$ and CNO chains from the latest SSM. . . . . . . . . . . . . . . 33

3.2 Comparison of the SSM BS05(OP) predictions for the neutrino rates and fluxes to the results reported by solar neutrino experi-

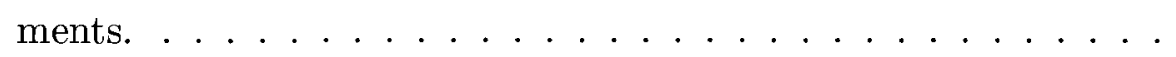

4.1 A schematic of the SNO detector. . . . . . . . . . . 43

5.1 Distribution of the difference between the raw livetimes measured by the $10 \mathrm{MHz}$ and $50 \mathrm{MHz}$ clocks. . . . . . . . . 62

5.2 Distribution of the $10 \mathrm{MHz}$ minus PGT estimated raw livetimes. . 64

5.3 Distribution of the combined deadtime for the data set during the NCD phase. ....................... 67

5.4 Distribution of the difference between the cut corrected livetimes from LSP and PGT. . . . . . . . . . . . . . 70

5.5 Corrected livetime of the NCD data set as a function of solar zenith

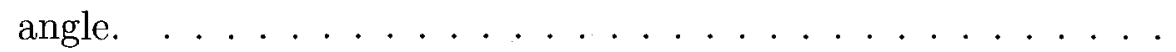

7.1 Radial distribution of the neutrino production inside the Sun. . . 102 
7.2 Before SNO-III: SNO-only neutrino oscillation confidence level contours. . . . . . . . . . . . . . . . . 132

7.3 After SNO-III: SNO-only neutrino oscillation confidence level con-

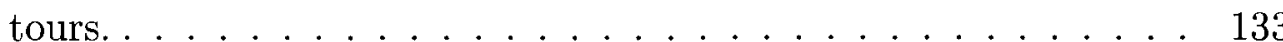

7.4 Before SNO-III: Global solar and global solar+KamLAND neutrino oscillation confidence level contours. . . . . . . . . . . . 135

7.5 One dimensional projections of the $\Delta \chi^{2}$ function before results from the SNO-III phase. . . . . . . . . . . . . 137

7.6 After SNO-III: Global solar and global solar+KamLAND neutrino oscillation confidence level contours. . . . . . . . . . . . . . 138

7.7 One dimensional projections of the $\Delta \chi^{2}$ function after results from the SNO-III phase. . . . . . . . . . . . . . . . 139

8.1 ${ }^{8} \mathrm{~B}$ neutrino survival probability $P_{e e}$ after propagation inside the

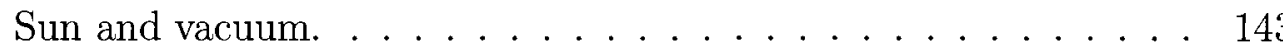

8.2 SNO-II CC kinetic energy spectrum compared with the LMA prediction. . . . . . . . . . . . . . . . 144

8.3 Survival probability $\bar{P}_{e e}^{\odot}$ for ${ }^{8} \mathrm{~B}$ electron neutrino. . . . . . . . . . 148

8.4 Survival probability $\bar{P}_{e e}^{\odot}$ for ${ }^{8} \mathrm{~B}$ electron neutrino projected in the $\sin ^{2} 2 \alpha-R_{\Delta}$ parameter space. . . . . . . . . . . . . . . 149

8.5 Survival probability $P_{e e}^{\ominus}$ for a pure electron neutrino state traversing the Earth at the nadir angle $\eta=90^{\circ} \ldots \ldots \ldots 151$

8.6 Survival probabilities $P_{e e}^{\ominus}$ for a pure electron neutrino state traversing the Earth for day, night and day-nigh average. . . . . . . . . 152 
8.7 Transition probabilities $P_{a e}^{\ominus}$ for a pure active neutrino state traversing the Earth for day, night and day-nigh average. . . . . . . . . 153

8.8 Transition probabilities $P_{s e}^{\ominus}$ for a pure sterile neutrino state traversing the Earth for day, night and day-nigh average. . . . . . . . . 154

8.9 Survival probabilities $P_{e e}^{\oplus}$ for the ${ }^{8} \mathrm{~B}$ and hep neutrinos for the SNOI phase for both day and night. . . . . . . . . . . . . . 157

8.10 Before SNO-III: SNO-only $\chi^{2}$ confidence level contours on the sterile neutrino parameter space for fixed ${ }^{8}$ B flux. . . . . . . . . 160

8.11 Before SNO-III: SNO-only $\chi^{2}$ confidence level contours on the sterile neutrino parameter space for free ${ }^{8} \mathrm{~B}$ flux. . . . . . . . . . . 161

8.12 Before SNO-III: One dimensional projections of the $\Delta \chi^{2}$ function on the $\sin ^{2} 2 \alpha$ and $R_{\Delta}$ axis from the SNO-only fit. . . . . . . . 162

8.13 After SNO-III: SNO-only $\chi^{2}$ confidence level contours on the sterile neutrino parameter space for fixed ${ }^{8}$ B flux. . . . . . . . . . . . . 164

8.14 After SNO-III: SNO-only $\chi^{2}$ confidence level contours on the sterile neutrino parameter space for free ${ }^{8}$ B flux. . . . . . . . . . . 165

8.15 After SNO-III: One dimensional projections of the $\Delta \chi^{2}$ function on the $\sin ^{2} 2 \alpha$ and $R_{\Delta}$ axis from the SNO-only fit. . . . . . . . 166

8.16 Global $\chi^{2}$ confidence level contours in the sterile neutrino parameter space for the partial SNO-II phase and fixed ${ }^{8} \mathrm{~B}$ flux. . . . . . 169

8.17 Global $\chi^{2}$ confidence level contours in the sterile neutrino parameter space for the partial SNO-II phase and free ${ }^{8}$ B flux. . . . . . . 170

8.18 Global $\chi^{2}$ confidence level contours of the sterile neutrino parameter space for the full SNO-II phase and fixed ${ }^{8} \mathrm{~B}$ flux. . . . . . . . 173 
8.19 Global $\chi^{2}$ confidence level contours of the sterile neutrino parameter space for the full SNO-II phase and free ${ }^{8} \mathrm{~B}$ flux. . . . . . . . . . 174

8.20 One dimensional projections of the $\Delta \chi^{2}$ function on $\sin ^{2} 2 \alpha$ and $R_{\Delta}$ axis for the global solar analyses. . . . . . . . . . . 175

8.21 Global $\chi^{2}$ confidence level contours of the sterile neutrino parameter space after the SNO-III phase and for fixed ${ }^{8}$ B flux. . . . . . . . 177

8.22 Global $\chi^{2}$ confidence level contours of the sterile neutrino parameter space after the SNO-III phase and for free ${ }^{8} \mathrm{~B}$ flux. . . . . . . . . 178

8.23 One dimensional projections of the $\Delta \chi^{2}$ function on $\sin ^{2} 2 \alpha$ and $R_{\Delta}$ axis for the global solar analyses after SNO-III. . . . . . . . . . 179

8.24 The kinetic energy CC spectra from the SNO-II phase with the latest LMA and sterile model predictions. . . . . . . . . . . 181

9.1 ${ }^{8} \mathrm{~B}$ electron neutrino survival probabilities for the Sun and vacuum for $\sin ^{2} \theta_{13}=0, \sin ^{2} \theta_{13}=0.01, \sin ^{2} \theta_{13}=0.04$ and $\sin ^{2} \theta_{13}=0.06$. . 187

9.2 SNO-I only neutrino oscillation contours from the EML analysis. . 190

9.3 SNO-I only neutrino oscillation contours from the $\chi^{2}$ analysis. . . 191

9.4 SNO only neutrino oscillation contours from the EML analysis. . . 192

B.1 Pulls of systematic uncertainties from the SNO-only EML analysis. 202 


\section{Chapter 1}

\section{Introduction}

The story of the neutrino starts in 1930 when Wolfgang Pauli postulated its existence in order to explain the problems of missing energy and spin statistics for some nuclei $\beta$ decays. The missing energy problem was first reported by Chandwick in 1914 when he discovered that the continuous electron spectrum from radioactive $\beta$ decays contrasted with the monoenergetic decay products from $\alpha$ and $\gamma$ decays. The problem of spin statistic arise from a picture of nuclei being composed of protons and electrons in early atomic models. As a solution to these two problems, in his public letter submitted at a physics conference in Tübingen [1] on the $4^{\text {th }}$ of December 1930, Pauli proposed a new, neutral, weakly interacting spin $1 / 2$ particle, which he originally named a neutron. The elusive particle is emitted in $\beta$ decay, thus share the total energy with the electron, which explains the continuous energy spectrum of $\beta$ decay. For the spectrum to be continuous, this particle must be very light and Pauli set the first limit on the neutrino mass to be $1 \%$ of the proton mass. It was assumed to be fermion - the spin $1 / 2$ particle would respect the Pauli exclusion principle and resolve the spin statistic problem 
in atomic nuclei.

After the neutron was discovered by Chadwick in 1932, Enrico Fermi renamed Paulis's particle the neutrino, as we know it today. In 1934, Fermi formulated a theory of $\beta$ decay, which is presently known as Fermi's theory $[2,3]$. He proposed that in $\beta$ decay, nuclei of atoms are transformed into different nuclei when a neutron disintegrates into a proton, an electron and a neutrino:

$$
n \rightarrow p+e^{-}+\bar{\nu}_{e},
$$

where nowadays Fermi's neutrino is known to be electron antineutrino.

It follows from Fermi's theory that neutrinos can be observed by detecting in coincidence positron and neutron events produced in the inverse $\beta$ decay:

$$
\bar{\nu}_{e}+p \rightarrow n+e^{+} .
$$

Application of this reaction led to the next milestone in neutrino physics: the discovery of the neutrino. Neutrino was first observed in 1956 by F. Reines and C. L. Cowan in their experiments using antineutrinos from a nuclear reactor [4]. For this breakthrough Reines was awarded the Nobel prize of physics in 1995. Unfortunately, Cowan did not live long enough to be awarded the honour.

With the formulation of the Standard Model (SM) of particle physics in the late 1960's by S. Weineberg [5] and A. Salam [6]; followed by its great success in the following decades while its predictions ${ }^{1}$ were confirmed by many experiments, neutrinos have been left massless. However, in 1962, Z. Maki, N. Nakagawa and S.

\footnotetext{
${ }^{1}$ The Higgs boson, which is necessary for the mass generating mechanism within the electroweak sector, is the only missing piece in the SM. It might be discovered at the Large Hadron Collider at CERN.
} 
Sakata [7] proposed a model with neutrinos being mixed states of massive eigenstates. The implication of this model was the prediction of a neutrino oscillation quantum phenomenon that could be confirmed with the detection of a neutrino in a flavour eigenstate that differs from its originally weakly produced state, due to the mixing of its mass eigenstates.

Neutrino oscillations were first observed in atmospheric neutrinos by the SuperKamiokande experiment in 1998 [8]. This was the first direct evidence of physics that goes beyond the scope of the minimal Standard Model. Indirect evidences of neutrino flavour conversion also came from various experiments [9-13] that observed a deficit of electron neutrinos produced by thermonuclear fusion reactions taking place in the core of the Sun. The Sudbury Neutrino Observatory was the first experiment to measure distinctively the total solar fluxes of electron neutrino and all known active neutrino flavours [14]. This milestone unambiguously proved that solar electron neutrinos do change flavour during their transit to the Earth.

But the story of neutrinos does not end here. There are many remaining questions regarding physics of this elusive particle. The fundamental question concerned with the origin of the neutrino mass could solve a mystery whether this particle is a Dirac or Majorana fermion. What is its mass? How many neutrino species are there in our Universe? Why does nature seems to be made of more matter than antimatter; and is there a link with possible $\mathrm{CP}$ violation in the leptonic sector? What constitutes the dark matter? All these questions inspire nowadays many particle physicists worldwide. Neutrinos which constantly pass through our planet, come from the Sun, distant stars, supernovae, cosmic rays and even those that were created right after the Big Bang. The relic neutrinos from the Big Bang almost lost all of their energy since their time of creation and 
surround us quietly waiting for a new insight in human thinking that could lead to their detection. Neutrino energy spans from $10^{-4} \mathrm{eV}$ for those produced by cosmic neutrino background and up to $10^{12} \mathrm{GeV}$ for those originated from active galactic nuclei and gamma ray bursts. Even higher energies are proposed in some cosmological models with topological defects, which were formed right after the phase of inflation of the Universe.

In this thesis, a quest to measure the fundamental properties of this intriguing particle is presented. Data samples collected by the Sudbury Neutrino Observatory, and other solar neutrino experiments, are used to investigate the mixing parameters of active and sterile neutrinos.

Glossary of all the acronyms defined within this thesis can be found in Appendix B. 


\section{Chapter 2}

\section{Physics of Neutrinos}

In this chapter, the focus is on the main features of neutrino physics that go beyond the minimal Standard Model of particle physics, with the emphasis on neutrino mixing model and special scenarios that are used in our analyses.

\subsection{Neutrinos in the Standard Model}

The Standard Model (SM) of particle physics is a gauge theory built within the framework of quantum field theory to describe elementary particles and their interactions $^{1}$. The local symmetry group of the $\mathrm{SM}$ is $\mathrm{SU}(3)_{\mathrm{C}} \times \mathrm{SU}(2)_{\mathrm{L}} \times \mathrm{U}(1)_{\mathrm{Y}}$, where $\mathrm{C}$ stands for colour, $\mathrm{L}$ for left-handed chirality and $\mathrm{Y}$ for weak hypercharge. Each symmetry group determines the interactions between the particles carrying the charge of that group and the number of vector gauge bosons mediating that interaction. The local $\mathrm{SU}(3)_{\mathrm{C}}$ group determines the strong interaction, which is mediated by eight massless gluons, between the particles carrying colour (quarks and gluons). All fermions (both quarks and leptons) participate in electroweak

\footnotetext{
${ }^{1}$ Gravitational interaction is not included in the Standard Model.
} 
interactions, which are related to $\mathrm{SU}(2)_{\mathrm{L}} \times \mathrm{U}(1)_{\mathrm{Y}}$ gauge group and mediated by the three massive bosons $\left(\mathrm{W}^{ \pm}\right.$and $\mathrm{Z}^{0}$ ) and one massless boson $(\gamma)$. Neutrinos are neutral leptons meaning that they only carry weak hypercharge and therefore do not participate in either strong or electromagnetic interactions. In other words, they are singlets with respect to the $\mathrm{SU}(3)_{\mathrm{C}}$ and $\mathrm{U}(1)_{\mathrm{EM}}$ groups.

In the SM, free fermion fields $\psi$ with mass $m$ are represented by four-component spinors satisfying the Dirac equation:

$$
i\left(\gamma^{\mu} \partial-m\right) \psi=0
$$

where $\gamma^{\mu}$ are Dirac $\gamma$ matrices. The fields $\psi=\psi_{R}+\psi_{L}$ can be split into chiral right-handed $\psi_{R}$ and left-handed $\psi_{L}$ components. For massless fermions, Equation (2.1) can be expressed in a decoupled form:

$$
\begin{aligned}
i \gamma^{\mu} \partial \psi_{R} & =0 \\
i \gamma^{\mu} \partial \psi_{L} & =0
\end{aligned}
$$

Thus massless fermions can be represented by a two component single chiral-field: $\psi_{R}$ or $\psi_{L}$.

In the SM, left-handed chirality fermion fields within the electroweak $\mathrm{SU}(2)_{\mathrm{L}} \times$ $\mathrm{U}(1)_{\mathrm{Y}}$ are grouped into weak isospin doublets. For three generations of quarks and leptons, these doublets are given as:

$$
Q_{\ell}=\left(\begin{array}{c}
p_{L} \\
q_{L}
\end{array}\right), \quad L_{\ell}=\left(\begin{array}{c}
\nu_{\ell L} \\
\ell_{L}
\end{array}\right),
$$

where $p$ denotes up-type $(u, c$ and $t)$ and $q$ denotes down-type $(d, s$ and $b)$ quarks; 
while $\ell=e, \mu$ and $\tau$ stands for the three generations of leptons. The right-handed components of the fermion fields do not have weak hypercharge, thus they are singlets under the $\mathrm{U}(1)_{Y}$ group. This is important in respect to neutrinos and a possible mechanism of mass generation, because the SM assumes that neutrinos have only left-handed fields ${ }^{2}$. However, it was discovered by E. Majorana in 1937 [15], that neutral fermions can be described by a single chirality field and its charged conjugate field (e.g $\psi_{L}$ and $\left.\psi_{L}^{C}\right)$ as:

$$
\psi=\psi_{L}+\psi_{L}^{C}
$$

Hence neutral fermion of this type is its own antiparticle:

$$
\psi=\psi^{C}
$$

The neutrino is the only neutral fermion known in the SM, whether it is a Dirac or a Majorana particle is still an unanswered question.

In the SM, fermion masses arise from the Higgs boson-fermion Yukawa interactions, which are given by the mass terms of the electroweak Lagrangian:

$$
\mathcal{L}_{\mathrm{M}}=-Y^{d} \bar{Q}_{L} \phi D_{R}-Y^{u} \bar{Q}_{L} \tilde{\phi} U_{R}-Y^{\ell} \bar{L}_{L} \phi L_{R}+\text { h.c. }
$$

Here $Y$ is the Yukawa coupling, $\phi$ is the Higgs field, $D_{R}$ and $U_{R}$ are right-handed singlets for the up- and down-type quark fields, while $L_{R}$ denotes right-handed fields for the charged leptons. Since neutrino fields do not have right-handed components, neutrinos cannot acquire a mass from the Yukawa interactions due

\footnotetext{
${ }^{2}$ Correspondingly, their antiparticles antineutrinos have only right-handed chiral fields.
} 
to the absence of the terms in the form $Y^{\ell} \bar{L}_{L} \phi \nu_{R}$, thus it remains massless. A massive neutrino would represent some new physics beyond the scope of the SM.

\subsection{Neutrino Mass}

Massive neutrinos are plausible and would require an extension of the SM model. It has been already mentioned that the non-existence of a right-handed neutrino field was an assumption within the SM. If one makes a minimal modification of the SM by introducing right-handed neutrino fields $\nu_{R}$, it follows from the Yukawa interactions that a neutrino can acquire its mass. These right-handed neutrinos are singlets within the SM gauge group; they do not carry any weak hypercharge, therefore they are called sterile neutrinos. With the new field $\nu_{R}$, the Lagrangian mass term for neutrinos is expressed as:

$$
\mathcal{L}_{\mathrm{M}_{\nu}}=-Y^{\nu} \bar{\nu}_{L} \tilde{\phi} \nu_{R}-\frac{1}{2} M \bar{\nu}_{R}^{c} \nu_{R}+\text { h.c. }
$$

The bare mass term in the Lagrangian is known as a Majorana mass term and allows a field without SM charges. However, this mechanism for the neutrino mass generation, generally, does not explain why neutrinos have tiny masses ${ }^{3}$. The bare mass term is not suppressed of having any possible mass. If a small neutrino mass arise from the small Yukawa coupling in the first (Dirac) mass term, that raises a question why this coupling is extremely smaller for neutrinos than for other fermions.

The other possibility for the neutrino mass originate in the assumption that the SM is an effective low energy theory of Nature, which is valid for energy scales

\footnotetext{
${ }^{3}$ The best limit from cosmology on the sum of neutrino masses is $0.3 \mathrm{eV}[16,17]$.
} 
$E<<\Lambda_{\mathrm{NP}}$, where $\Lambda_{\mathrm{NP}}$ describes the energy scale for new physics (NP). The rare effects from new physics (neutrino mass, proton decay, etc...) can be parametrized by adding higher order dimensional operators to the SM Lagrangian [18]:

$$
\mathcal{L}=\mathcal{L}^{\mathrm{SM}}+\frac{1}{\Lambda_{\mathrm{NP}}} \mathcal{L}_{5}+\frac{1}{\Lambda_{\mathrm{NP}}^{2}} \mathcal{L}_{6}+\ldots
$$

There is only one term in the dimension-5 operator $\mathcal{L}_{5}=L_{L} \phi L_{L} \phi$, which breaks the lepton number by two units leading to the neutrino mass of the form:

$$
m_{\nu}=\frac{\langle\phi\rangle\langle\phi\rangle}{\Lambda_{\mathrm{NP}}},
$$

where $\langle\phi\rangle$ is the vacuum expectation value for the Higgs field and represents the mass of the electroweak breaking scale. In this case, a tiny neutrino mass arises from the large mass scale $\Lambda_{\mathrm{NP}}$. Thus neutrino masses are the lowest order effects for possible new physics at high-energy scale. In addition, lepton mixing and CP violation arise naturally since both lepton number and lepton flavour symmetry are broken.

In the scenario of the famous see-saw mechanism [19-21], new heavy sterile neutrino fields with the mass $M>>\langle\phi\rangle$ are added to the SM, leading to the Lagrangian of the form shown in Equation (2.7). The see-saw mechanism generate the mass matrix for the neutrino fields:

$$
M_{\nu}=\left(\begin{array}{cc}
0 & m_{D} \\
m_{D} & M
\end{array}\right),
$$

where $m_{D}$ are the Dirac mass terms arising from the first term of Equation (2.7). Diagonalization of the mass matrix $M_{\nu}$ leads to three light neutrino mass eigen- 
statates with mass matrix $m_{\nu}=m_{D}^{T} M^{-1} m_{D}$, and heavy sterile states with mass matrix $M$. The large scale of $M$ is attributed to the NP scale $\Lambda_{\mathrm{NP}}$, therefore the SM remains as a good effective theory in the low energy regime with the three light neutrinos.

\subsection{Neutrino Mixing}

The number of neutrino species in the SM is defined by the width of the $Z^{0}$ boson, the mediator of the neutral-current weak interaction. It was shown by the experimental results of LEP (the latest report from the LEP experiment can be found in Reference [22]) that there are three active neutrino flavours: $\nu_{e}, \nu_{\mu}$ and $\nu_{\tau}$. Each neutrino flavour is determined by a corresponding charged lepton $(e, \mu$ and $\tau)$ to which neutrinos couple via the charged-current weak interaction mediated by the $W^{ \pm}$bosons, thus the three neutrino flavours represent interaction states. Whereas, from the same processes, the different charged leptons are identified only by their mass. Hence, charged lepton mass eigenstates must be identical to their interaction eigenstates and the mixing between the charged leptons is not permitted. If neutrinos are massive, nothing prevents them from mixing, therefore their mass eigenstates can differ from their interaction eigenstates.

More than three neutrino species are possible under the assumption that these new species are singlets within the SM gauge group. Thus, in general, if neutrinos do mix, each of $N$ left-handed components of neutrino fields $\nu_{\alpha L}$, where $\alpha=$ $e, \mu, \tau, s_{1}, s_{2} \ldots s_{N-3}$ ( $s$ stands for sterile), can be expressed for the three active flavours and $N-3$ sterile states as a linear combination of $N$ left-handed mass 
eigenstates $\nu_{k L}$ with masses $m_{k}$ [23]:

$$
\nu_{\alpha L}=\sum_{k=1}^{N} U_{\alpha k} \nu_{k L},
$$

where $k=1, . . N$ and $U$ is a unitary mixing matrix. For the case of three active neutrino flavours this matrix is called the Pontecorvo-Maki-Nakawa-Sakata (PMNS) matrix $U^{P M N S}$ :

$$
U^{P M N S}=\left(\begin{array}{ccc}
c_{12} c_{13} & s_{12} c_{13} & s_{13} e^{-i \delta} \\
-s_{12} c_{23}-c_{12} s_{23} s_{13} e^{i \delta} & c_{12} c_{23}-s_{12} s_{23} s_{13} e^{i \delta} & s_{23} c_{13} \\
s_{12} s_{23}-c_{12} c_{23} s_{13} e^{i \delta} & -c_{12} s_{23}-s_{12} c_{23} s_{13} e^{i \delta} & c_{23} c_{13}
\end{array}\right)
$$

with the three mixing angles $c_{i j}=\cos \theta_{i j}$ and $s_{i j}=\sin \theta_{i j}$ and one CP violating phase $\delta$. In addition, if neutrinos are their own antiparticles (i.e. Majorana particles) $\nu_{\alpha}=\bar{\nu}_{\alpha}$, there are two additional Majorana phases $\lambda_{1}$ and $\lambda_{2}$ entering the mixing matrix as:

$$
U=U^{P M N S} \operatorname{diag}\left(1, e^{i \lambda_{1}}, e^{i \lambda_{1}}\right)
$$

\subsection{Neutrino Oscillation}

We are interested in the observable effects of the neutrino mixing shown in Equation (2.11), knowing that neutrino flavour $\nu_{\alpha}$ is exclusively identified by a charged lepton from the charged-current interaction during neutrino production and detection process. Sterile states cannot be produced from weak interactions, therefore we are looking in the active neutrino flavour state $\nu_{\alpha}$, which is produced from a 
charged lepton $\ell_{\alpha}^{-}$or together with a charged antilepton $\ell_{\alpha}^{+}$as a coherent superposition of massive neutrino states:

$$
\left|\nu_{\alpha L}\right\rangle=\sum_{k} U_{\alpha k}^{*}\left|\nu_{k L}\right\rangle, \alpha=e, \nu, \tau
$$

This is a standard approach in classical theory of neutrino mixing and oscillations [24-26] where massive neutrino states are assumed to be plane waves. Further information on the wave packet treatment and derivation on neutrino states within quantum filed theory can be found, for example, in Reference [27].

The massive neutrino states $\left|\nu_{k L}\right\rangle$, as eigenstates of the Hamiltonian, are produced with the definite energies (natural system of units is assumed $c=\hbar=1$ ):

$$
E_{k}=\sqrt{\vec{p}^{2}+m_{k}^{2}}
$$

It follows from the Schrödinger equation describing plane wave state neutrino propagation in vacuum, that after a time interval $t$, the flavour state $\nu_{\alpha L}(t)$ evolved as:

$$
\left|\nu_{\alpha L}(t)\right\rangle=\sum_{k} U_{\alpha k}^{*} e^{-i E_{k} t}\left|\nu_{k L}\right\rangle
$$

From the unitarity of the mixing matrix $U$, the mass eigenstates can be expressed as a linear combination of the flavour states:

$$
\left|\nu_{k L}\right\rangle=\sum_{\alpha} U_{\alpha k}\left|\nu_{\alpha L}\right\rangle
$$

Combining Equation (2.17) with Equation (2.16), the final neutrino flavour state 
at the time $t$ is given by:

$$
\left|\nu_{\alpha L}(t)\right\rangle=\sum_{\beta}\left(\sum_{k} U_{\alpha k}^{*} e^{-i E_{k} t} U_{\beta k}\right)\left|\nu_{\beta L}\right\rangle .
$$

Equation (2.18) shows that a neutrino produced in the flavour state $\alpha$ after propagating a certain distance in vacuum, becomes a superposition of all three flavour eigenstates. Hence, it can be detected via charged-current interaction processes in a different flavour $\beta$, which is known as the phenomenon of neutrino oscillations. Neutrino oscillations were first proposed by Bruno Pontecorvo in $1956[28]^{4}$. A transition probability $P_{\nu_{\alpha} \rightarrow \nu_{\beta}}$ for this to occur is determined from the transition amplitudes:

$$
A_{\nu_{\alpha} \rightarrow \nu_{\beta}}=\left\langle\nu_{\beta} \mid \nu_{\alpha}\right\rangle=\sum_{k} U_{\alpha k}^{*} e^{-i E_{k} t} U_{\beta k}
$$

as

$$
P_{\nu_{\alpha} \rightarrow \nu_{\beta}}=\left|A_{\nu_{\alpha} \rightarrow \nu_{\beta}}\right|^{2}=\sum_{k j} U_{\alpha k}^{*} U_{\beta k} U_{\alpha j} U_{\beta j}^{*} e^{-i\left(E_{k}-E_{j}\right) t} .
$$

The transition probability from this expression can be further simplified knowing that neutrinos are ultrarelativistic particles. Hence, energy eigenvalues from Equation (2.15) can be approximated by:

$$
E_{k} \simeq E+\frac{m_{k}^{2}}{2 E}, E=\left|\vec{p}^{2}\right| .
$$

Therefore, a difference between the two energy eigenvalues is given as:

$$
E_{k}-E_{j}=\frac{\Delta m_{k j}^{2}}{2 E},
$$

\footnotetext{
${ }^{4}$ The first neutrino oscillation phenomenon proposed by Bruno Pontecorvo described neutrino-antineutrino transitions.
} 
where the squared-mass difference $\Delta m_{k j}^{2}$ is defined as a difference between the squared masses of the two neutrino mass eigenstates:

$$
\Delta m_{k j}^{2}=m_{k}^{2}-m_{j}^{2}
$$

Since from the experiments, only the distance $L$ between the neutrino production and detection region is measured, and not the elapsed time $t$ of neutrino propagation, the following approximation, which is valid for ultrarelativistic particles, is used: $t=L$. Under this approximations, the final transition probability in vacuum is derived then as:

$$
P_{\nu_{\alpha} \rightarrow \nu_{\beta}}=\sum_{k j} U_{\alpha k}^{*} U_{\beta k} U_{\alpha j} U_{\beta j}^{*} \exp \left(-i \frac{\Delta m_{k j}^{2} L}{2 E}\right)
$$

This equation shows that transition probability is determined by the properties of neutrino: its mixing matrix $U$ and the squared-mass differences $\Delta m_{k j}^{2}$; and also by the ratio of production-detection point distance to neutrino energy $L / E$, that differs between various experiments. For a case when neutrino is detected in the same flavour in which it was produced $(\alpha=\beta)$, probability in the form

$$
P_{\nu_{\alpha} \rightarrow \nu_{\alpha}}=1-P_{\nu_{\alpha} \rightarrow \nu_{\beta}}
$$

is called the survival probability.

Equation (2.25) can be expressed by separating the constant term originating from the elements of the mixing matrix, from the oscillatory term containing the 
phase of oscillations $-\Delta m_{k j}^{2} L / 2 E$ :

$$
P_{\nu_{\alpha} \rightarrow \nu_{\beta}}=\sum_{k}\left|U_{\alpha k}\right|^{2}\left|U_{\beta k}\right|^{2}+2 R e \sum_{k j, k>j} U_{\alpha k}^{*} U_{\beta k} U_{\alpha j} U_{\beta j}^{*} \exp \left(-i \frac{\Delta m_{k j}^{2} L}{2 E}\right),
$$

where a wavelength for oscillation, known as the oscillation length is defined as:

$$
\Lambda_{k j}=\frac{4 \pi E}{\Delta m_{k j}^{2}}
$$

When coherence of different neutrino propagation states is lost, the oscillatory terms in Equation (2.26) are averaged out, leaving the constant term in the transition probability only:

$$
P_{\nu_{\alpha} \rightarrow \nu_{\beta}}=\sum_{k}\left|U_{\alpha k}\right|^{2}\left|U_{\beta k}\right|^{2} .
$$

The same average probability is used when detector resolution is not sufficient enough to discriminate between various phases $-\Delta m_{k j}^{2} L / 2 E$.

It should be noted that experiments measuring transition (neutrino appearance experiments) or survival probability (neutrino disappearance experiments) cannot differentiate whether neutrinos are Dirac fermions or Majorana particles, since Majorana phases from Equation (2.13) cancel in the derivation of these probabilities.

\subsubsection{Two-Neutrino Approximation}

The simplest assumption towards the derivation of the transition probabilities is the two-neutrino approximation, which is often used by many experiments that do not have sensitivity to the effect of three-neutrino mixing to interpret their results. The two-flavour neutrino states considered in this approximation could be pure 
neutrino flavours $\left(\nu_{e}, \nu_{\mu}\right.$ or $\left.\nu_{\tau}\right)$ produced from charged-current reaction processes or linear combinations of this pure states. For the case of solar neutrinos, which are used in our analyses, we consider $\nu_{e}$ produced in the solar core and the linear combination $\nu_{a}$ for the final state given by:

$$
\nu_{a}=c_{\nu} \nu_{\mu}+c_{\tau} \nu_{\tau}
$$

where $\left|c_{\nu}\right|^{2}+\left|c_{\tau}\right|^{2}=1$. This is justified through the incapability of a detector to differentiate between $\nu_{\mu}$ and $\nu_{\tau}$ neutrino flavours, and by the experimental fact that the probability of the third mass eigenstate to mix into $\nu_{e}$ flavour is small [29]. In this case, $\nu_{e}$ and $\nu_{a}$ neutrino flavour states are linear combinations of the two neutrino mass eigenstates $\nu_{1}$ and $\nu_{2}$, with the squared-mass difference:

$$
\Delta m^{2} \equiv \Delta m_{21}^{2}=m_{2}^{2}-m_{1}^{2} .
$$

The two mass eigenstates mix into the two flavour states with the coefficients from the unitary mixing matrix given as:

$$
U=\left(\begin{array}{cc}
\cos \theta & \sin \theta \\
-\sin \theta & \cos \theta
\end{array}\right)
$$

where $\theta \equiv \theta_{12} \in[0, \pi / 2]$ is the mixing angle. From Equation (2.26), the two neutrino transition probability $P_{\nu_{\alpha} \rightarrow \nu_{\beta}}$ is derived as:

$$
P_{\nu_{\alpha} \rightarrow \nu_{\beta}}=\frac{1}{2} \sin ^{2} 2 \theta\left(1-\cos \left(\frac{\Delta m^{2} L}{2 E}\right)\right),
$$


and the survival probability $P_{\nu_{\alpha} \rightarrow \nu_{\alpha}}$ is:

$$
P_{\nu_{\alpha} \rightarrow \nu_{\alpha}}=1-\sin ^{2} 2 \theta \sin ^{2}\left(\frac{\Delta m^{2} L}{4 E}\right) .
$$

The phase averaged transition probability is given as:

$$
\bar{P}_{\nu_{\alpha} \rightarrow \nu_{\beta}}=\frac{1}{2} \sin ^{2} 2 \theta,
$$

where it is obvious that this probability cannot be greater than $1 / 2$, and consequently the opposite is true for the corresponding survival probability due to unitarity condition:

$$
P_{\nu_{\alpha} \rightarrow \nu_{\alpha}}+P_{\nu_{\alpha} \rightarrow \nu_{\beta}}=1 \text {. }
$$

\subsubsection{Weakly Mixed Sterile Neutrino Model}

Another neutrino mixing model has been used in this dissertation as a model describing a system with two active and a single weakly mixed sterile neutrino. This model was proposed by A. Yu. Smirnov and P. C. de Holanda in Reference [30] as an attempt to explain a non-observation of some predicted effects from the two-neutrino mixing hypothesis. More details on a motivation to use this model and its impact on solar neutrino survival probabilities will be presented later in Chapter 8.

In this model, a system of three neutrinos given in flavour eigenstate basis is composed of an electron $\nu_{e}$ and an active $\nu_{a}$ neutrino flavours, plus one weakly mixed sterile neutrino $\nu_{s}$. The active neutrino flavour $\nu_{a}$ is a linear combination of muon $\nu_{\mu}$ and tau $\nu_{\tau}$ neutrinos (as given in Equation (2.29)). There are three 
mass eigenstates $\nu_{1}, \nu_{2}$ and $\nu_{0}$ that mix into these flavour states as:

$$
\left(\begin{array}{c}
\nu_{s} \\
\nu_{e} \\
\nu_{a}
\end{array}\right)=U\left(\begin{array}{l}
\nu_{0} \\
\nu_{1} \\
\nu_{2}
\end{array}\right)
$$

where $U$ is the unitary mixing matrix of this form:

$$
U=\left(\begin{array}{ccc}
\cos \alpha & -\sin \alpha & 0 \\
\cos \theta \sin \alpha & \cos \theta \cos \alpha & \sin \theta \\
-\sin \theta \sin \alpha & -\sin \theta \cos \alpha & \cos \theta
\end{array}\right) .
$$

Masses of these mass eigenstates have the following hierarchy:

$$
m_{1}<m_{0}<m_{2}
$$

The assumptions from the model provide that the two active neutrino states $\left(\nu_{e}\right.$ and $\nu_{a}$ ) are described by the mixing parameters $\Delta m_{21}^{2} \equiv \Delta m^{2}$ and $\theta_{12} \equiv \theta$ from the two-neutrino mixing parameter space. The sterile neutrino $\nu_{s}$ mainly populate the mass eigenstate $\nu_{0}$ that is characterized by the $\cos \alpha$ element of the matrix element $U_{11}$. It mixes weakly (with the coefficient $\sin \alpha$ ) with the two active neutrinos in the mass eigenstate $\nu_{1}$, and does not populate the mass eigenstate $\nu_{2}$. In our analyses, the sterile neutrino mixing parameters are assumed to be within this range:

$$
\begin{aligned}
\Delta m_{01}^{2}= & m_{0}^{2}-m_{1}^{2}=[0,1.8] \times 10^{-5} \mathrm{eV}^{2}, \\
& \sin ^{2} 2 \alpha=\left[10^{-5}, 10^{-2}\right] .
\end{aligned}
$$

Parameters outside this range would either produce non-observable effects in solar 
neutrino data (for smaller mixing angle $\alpha$ ), or would have a significant impact on measurements reported by various solar neutrino experiments (for large $\alpha$ and/or large $\left.\Delta m_{01}^{2}\right)$ and have already been ruled out. It is also useful to define a parameter $R_{\Delta}$ that is the ratio of the neutrino squared-mass differences $\Delta m_{01}^{2}$ and $\Delta m_{21}^{2}$, following a definition for sterile neutrino parameter space from Reference [30]:

$$
R_{\Delta}=\frac{\Delta m_{21}^{2}}{\Delta m_{01}^{2}}
$$

\subsection{Matter Effect}

While passing through matter, active neutrino flavours scatter with electrons and nucleons. The mean free path for the incoherent scattering of neutrinos via charged-current and neutral-current interactions, through which their momentum and energy are being changed, is extremely large due to the weakness of these interactions. The cross-sections for an incoherent scattering process $\sigma_{\text {ich }}$ is proportional to the Fermi constant $G_{\mathrm{F}}$, leading to the mean free path for neutrinos inside the medium of $\lambda_{\text {m.f.p }} \sim 1 / N \sigma_{\text {ich }}$, where $\mathrm{N}$ is the number density of the target particles inside the matter of interest (e.g. $\lambda_{\text {m.f.p }} \sim 10^{17} \mathrm{~cm}$ for matter with a density of $1 \mathrm{~g} / \mathrm{cm}^{3}$ [31]). The other type of interaction experienced by neutrinos is the coherent forward elastic scattering on the particles inside the medium of propagations. It was discovered by Wolfenstein in 1978 [32] that effective potentials describing these coherent processes can alter neutrino mixing ${ }^{5}$. All active neutrino flavours interact with the electrons and nucleons in matter through neutral-current (NC) interaction exchanging $Z^{0}$ bosons. Due to electrical

\footnotetext{
${ }^{5}$ At that time, Wolfenstein used a wrong sign for the matter potential.
} 
neutrality of matter, there is an equal number of electron and proton scatterers, therefore their contribution to to the NC matter potential $V_{\mathrm{NC}}$ is cancelled. The remaining neutron contribution yields to the effective $\mathrm{NC}$ potential given by:

$$
V_{\mathrm{NC}}=-0.5 \sqrt{2} G_{\mathrm{F}} N_{n},
$$

where $N_{n}$ is the number density of neutrons for the medium of interest. In addition to the NC coherent scattering, only electron neutrinos experience the chargedcurrent elastic scattering where the effective potential is given by:

$$
V_{\mathrm{CC}}=\sqrt{2} G_{\mathrm{F}} N_{e},
$$

with the electron number density $N_{e}$.

The neutrino propagation equation inside matter, for a general case of three active and $n$ sterile neutrinos, using flavour eigenstate basis is given as:

$$
i \frac{d}{d t}\left(\begin{array}{c}
\nu_{e} \\
\nu_{\mu} \\
\nu_{\tau} \\
\nu_{s 1} \\
\cdots \\
\nu_{s n}
\end{array}\right)=\left(H_{v a c}+H_{m}\right)\left(\begin{array}{c}
\nu_{e} \\
\nu_{\mu} \\
\nu_{\tau} \\
\nu_{s 1} \\
\cdots \\
\nu_{s n}
\end{array}\right),
$$

with the vacuum part of the Hamiltonian expressed as follow:

$$
H_{v a c}=\frac{1}{2 E} U \operatorname{diag}\left(m_{1}^{2}, m_{2}^{2}, m_{3}^{2}, m_{s 1}^{2}, \ldots, m_{s n}^{2}\right) U^{\dagger}
$$


and the Hamiltonian in matter defined as:

$$
H_{m}=\operatorname{diag}\left(V_{\mathrm{CC}}+V_{\mathrm{NC}}, V_{\mathrm{NC}}, V_{\mathrm{NC}}, 0, \ldots 0\right)
$$

Since effective potentials change inside a medium with a varying density, there is no general analytic solution for the neutrino propagation equation given in Equation (2.43). Therefore, it has to be solved numerically in order to derive neutrino transition and survival probabilities for neutrinos traversing matter.

\subsubsection{MSW Effect for the Two-Neutrino Scenario}

For a two-neutrino case, the neutrino propagation equation derived from Equation (2.43) can be written as:

$$
i \frac{d}{d t}\left(\begin{array}{c}
\nu_{e} \\
\nu_{a}
\end{array}\right)=\left(A+\left(\begin{array}{cc}
-\frac{\Delta m^{2}}{4 E} C_{2 \theta}+V_{\mathrm{CC}} & \frac{\Delta m^{2}}{4 E} S_{2 \theta} \\
\frac{\Delta m^{2}}{4 E} S_{2 \theta} & \frac{\Delta m^{2}}{4 E} C_{2 \theta}
\end{array}\right)\left(\begin{array}{c}
\nu_{e} \\
\nu_{\mu}
\end{array}\right)\right)
$$

where $C_{2 \theta}=\cos 2 \theta$ and $S_{2 \theta}=\sin 2 \theta$. The diagonal matrix $A$ is

$$
A=\frac{m_{1}^{2}+m_{2}^{2}+V_{\mathrm{NC}}}{4 E} \operatorname{diag}(1,1)
$$

and hence does not affect oscillations, since it alters all neutrino phases equally, therefore it can be omitted in the calculations of the transition probabilities. The effective Hamiltonian in matter $H_{\text {eff }}$ is then given by:

$$
H_{e f f}=\left(\begin{array}{cc}
-\frac{\Delta m^{2}}{4 E} C_{2 \theta}+V_{\mathrm{CC}} & \frac{\Delta m^{2}}{4 E} S_{2 \theta} \\
\frac{\Delta m^{2}}{4 E} S_{2 \theta} & \frac{\Delta m^{2}}{4 E} C_{2 \theta}
\end{array}\right)
$$


In 1985, it was noted by Mikheyev and Smirnov [33,34] that neutrinos traveling through matter with varying density could enter into the region where resonant flavour transition can occur. This alteration of the initial neutrino flux is known as the Mikheyev-Smirnov-Wolfenstein (MSW) effect. It order to see when the MSW effect appears, it is instructive to diagonalize the effective Hamiltonian $H_{\text {eff }}$ in the effective mass eigenstate basis for the medium of propagation [31]:

$$
U_{m}^{\dagger} H_{e f f} U_{m}=\frac{\Delta m_{m}^{2}}{4 E} \operatorname{diag}(-1,1)
$$

The unitary mixing matrix in matter $U_{m}$ (c.f. Equation (2.31)) is expressed in terms of the effective mixing angles in matter $\theta_{m}$ given by:

$$
\tan 2 \theta_{m}=\frac{\Delta m^{2} \sin 2 \theta}{\Delta m^{2} \cos 2 \theta-2 E V_{\mathrm{CC}}}
$$

The effective squared-mass difference $\Delta m_{m}^{2}$ is expressed as:

$$
\Delta m_{m}^{2}=\sqrt{\left(\Delta m^{2} \cos 2 \theta-2 E V_{\mathrm{CC}}\right)^{2}+\left(\Delta m^{2} \sin 2 \theta\right)^{2}} .
$$

The MSW effect occurs at the resonant electron density $N_{e}^{\mathrm{R}}$, for which the denominator in Equation (2.50) equals zero so that:

$$
2 E V_{\mathrm{CC}}=2 \sqrt{2} E G_{\mathrm{F}} N_{e}^{\mathrm{R}}=\Delta m^{2} \cos 2 \theta
$$

At the resonance, the mixing is maximal $\left(\theta_{m}=\pi / 4\right)$ and it can lead to the complete transition $\nu_{e} \rightarrow \nu_{\alpha}$ between the two neutrino flavour states, thus to the maximal enhancement of neutrino oscillations. 
The MSW effect could alter the flavour eigenstates of neutrinos produced in the core of the Sun during their propagation towards the solar surface, and possibly through the Earth in their way to the detector. In general, maximal transition between flavour eigenstates can occur in matter even if neutrino mixing in vacuum is expected to be small.

\subsubsection{MSW Effect for the Sterile Scenario}

For the three neutrino system that is given in Equation (2.36), a survival $P_{\nu_{e} \rightarrow \nu_{e}}$ and transition $P_{\nu_{e} \rightarrow \nu_{a}}$ and $P_{\nu_{e} \rightarrow \nu_{s}}$ probabilities in matter are derived by solving a neutrino propagation equation expressed in flavour eigenstate basis as:

$$
i \frac{d}{d t}\left(\begin{array}{c}
\nu_{s} \\
\nu_{e} \\
\nu_{a}
\end{array}\right)=\left(H_{v a c}+H_{m}\right)\left(\begin{array}{c}
\nu_{s} \\
\nu_{e} \\
\nu_{a}
\end{array}\right)
$$

where a vacuum part of the Hamiltonian is defined by:

$$
H_{v a c}=\frac{1}{2 E} U\left(\begin{array}{ccc}
m_{0}^{2} & 0 & 0 \\
0 & m_{1}^{2} & 0 \\
0 & 0 & m_{2}^{2}
\end{array}\right) U^{\dagger}
$$

with $E$ being the neutrino energy. A matter part of the three neutrino Hamiltonian is given by:

$$
H_{m}=\left(\begin{array}{ccc}
0 & 0 & 0 \\
0 & V_{\mathrm{CC}}+V_{\mathrm{NC}} & 0 \\
0 & 0 & V_{\mathrm{NC}}
\end{array}\right),
$$


where $V_{\mathrm{CC}}$ and $V_{\mathrm{NC}}$ are the effective potentials for the matter with varying density, that arise from the electron and nucleon scatterers, respectively, as given in Equations (2.41) and (2.42).

It has been shown that for a particular case of neutrinos moving inside the Sun, the weakly mixed sterile energy eigenvalue crosses the energy level of the first mass eigenstate in matter $\left(\nu_{m 1}\right)$ only [30]. Thus, the second mass eigenstate $\left(\nu_{m 2}\right)$ decouples from the three neutrino system and its evolution is adiabatic. In other words, this state does not cross the energy levels of the other states and evolves into its vacuum state as $\nu_{m 2} \rightarrow \nu_{2}$. The propagation of the mass eigenstates $\nu_{m 0}$ and $\nu_{m 1}$ is described by the two-neutrino Hamiltonian:

$$
H_{d}=\left(\begin{array}{ll}
\lambda_{1} & \frac{\Delta m_{01}^{2}}{4 E} \sin 2 \alpha \cos \left(\theta-\theta_{m}\right) \\
\frac{\Delta m_{01}^{2}}{4 E} \sin 2 \alpha \cos \left(\theta-\theta_{m}\right) & \frac{m_{1}^{2}+m_{0}^{2}}{4 E}+\frac{\Delta m_{01}^{2}}{4 E} \cos 2 \alpha
\end{array}\right)
$$

where the $\lambda_{1}$ is the energy eigenvalue for the $\nu_{1 m}$ state, given by:

$$
\lambda_{1}=\frac{m_{1}^{2}+m_{2}^{2}}{4 E}+\frac{V_{\mathrm{CC}}-2 V_{\mathrm{NC}}}{2}-\frac{1}{4 E} \Delta m_{m}^{2}
$$

with the $C_{2 \theta}=\sin 2 \theta_{m}$ and $S_{2 \theta}=\cos 2 \theta_{m}$. The $\theta_{m}$ describes a mixing of the $\nu_{m 1}$ and $\nu_{m 2}$ system in matter as expressed in Equation (2.50), while the effective squared-mass difference $\Delta m_{m}^{2}$ is defined in Equation (2.51). A resonance condition for the MSW effect to occur is achieved when the diagonal matrix elements of the Hamiltonian $H_{d}$ are equal, leading to a following relation:

$$
2 E\left(V_{\mathrm{CC}}-2 V_{\mathrm{NC}}\right)=2 \sqrt{2} E G_{\mathrm{F}}\left(N_{e}^{\mathbf{R}}-N_{n}^{\mathbf{R}}\right)=\Delta m_{m}^{2}+\Delta m_{01}^{2}\left(1-R_{\Delta}+\cos 2 \alpha\right) .
$$




\subsection{Summary}

The possibility of massive neutrinos and neutrino mixing hypothesis have been presented in this chapter. In Chapters 7 and 8 , tests of neutrino mixing hypothesis performed by the Sudbury Neutrino Observatory under the assumptions of solar neutrinos being composed of either two active, or two active and one sterile neutrino flavour, are presented. For completeness of the analyses presented in this dissertation, the measurements of other solar neutrino experiments are also used. Solar neutrinos, which are described in the next chapter, are produced in the core of the Sun. They experience matter effect while propagating from the solar core to its surface, vacuum oscillations in their path towards the Earth and, again, are subjected to matter effect while traversing the Earth. Therefore, by using solar neutrino data, neutrino mixing parameters can be extracted. 


\section{Chapter 3}

\section{Solar Neutrinos}

Solar neutrinos are produced as $\nu_{e}$ flavour by thermonuclear fusion reactions in the core of the Sun. The theory of stellar nucleosynthesis via thermonuclear fusion reaction chains occurring in stellar cores was proposed by Hans Bethe [35]. Being extremely weakly interacting particles, neutrinos travel from the solar core to the Earth almost undisturbed. If detected, neutrinos can give us a glance at the Sun's interior in almost real time, compared to the light from the Sun that due to the extreme conditions in the solar interior reaches us tens of thousands years after being produced. The first historical interest in detection of solar neutrinos came from Raymond Davis and John Bachall in the early 1960's. Their goal was to prove whether the theory of stellar energy production via thermonuclear fusion reaction was correct or not.

The production of solar neutrinos, predictions of solar neutrino fluxes at the Earth, solar neutrino experiments, origin of the solar neutrino problem and its solution are summarized in this chapter. 


\subsection{Neutrino Production}

The Sun is powered by thermonuclear fusion reactions occurring in its core at temperatures of about $1.5 \times 10^{7} \mathrm{~K}$. It is a main sequence star according to the Hertzsprung-Russell diagram of stelar luminosity versus surface temperature, meaning that it is in hydrogen burning phase. It is estimated to subsist approximately 10 billion years. The resulting fusion products of burning hydrogen are a helium nucleus and two electron neutrinos. Energy released can either be in the form of photons or taken by neutrinos:

$$
4 p+2 e^{-} \rightarrow{ }^{4} \mathrm{He}+2 \nu_{e}+Q .
$$

The net released energy in this process equals $4 m_{p}+2 m_{e}-m_{4} \mathrm{He}=26.73 \mathrm{MeV}$, where $m_{p}, m_{e}$ and $m_{4} \mathrm{He}$ are the masses of the proton, electron and helium nucleus, respectively.

There are two main chains of reactions through which the final production of helium is achieved. The first is the $p p$ chain and the other is the so-called CNO cycle. Approximately, $98 \%$ of the energy release in solar thermonuclear reactions is produced by the $p p$ chain. In $99.6 \%$ of the cases, the $p p$ chain starts with the fusion of two protons producing a deuteron, a positron and an electron neutrino:

$$
p+p \rightarrow{ }^{2} \mathrm{H}+e^{+}+\nu_{e}+0.420 \mathrm{MeV} .
$$

This reaction is also known as the $p p$ reaction. The supplementing starting reaction of the $p p$ chain, occurring $0.4 \%$ of the time, is the pep reaction where a 
monoenergetic neutrino is produced:

$$
p+e^{-}+p \rightarrow{ }^{2} \mathrm{H}+\nu_{e}+1.442 \mathrm{MeV} .
$$

The $p p$ and $p e p$ reactions lead to:

$$
{ }^{2} \mathrm{H}+\mathrm{p} \rightarrow{ }^{3} \mathrm{He}+\gamma+5.494 \mathrm{MeV}
$$

which is then followed by three possible reactions to complete the $p p$ cycle. With a $85 \%$ branching ratio, the first reaction of this $p p$ sub-chain occurs, which is denoted as the $p p$ I chain:

$$
{ }^{3} \mathrm{He}+{ }^{3} \mathrm{He} \rightarrow{ }^{4} \mathrm{He}+2 \mathrm{p}+12.860 \mathrm{MeV} .
$$

The second reaction, which is extremely rare occurring at only the $2 \times 10^{-5 \%}$ level, produces the so-called hep neutrinos:

$$
{ }^{3} \mathrm{He}+\mathrm{p} \rightarrow{ }^{4} \mathrm{He}+\mathrm{e}^{+}+\nu_{\mathrm{e}}+18.770 \mathrm{MeV}
$$

The third reaction with a branching ratio of $15 \%$ proceeds with the fusion of ${ }^{3} \mathrm{He}$ and ${ }^{4} \mathrm{He}$ :

$$
{ }^{3} \mathrm{He}+{ }^{4} \mathrm{He} \rightarrow{ }^{7} \mathrm{Be}+\gamma+1.586 \mathrm{MeV} .
$$

It is followed with the branching chains known as the ppII (at 99.87\%) and ppIII (at $0.13 \%$ ). The reactions of the $p p I I$ chain are:

$$
{ }^{7} \mathrm{Be}+\mathrm{e}^{-} \rightarrow{ }^{7} \mathrm{Li}+\nu_{\mathrm{e}}+0.862 \mathrm{MeV},
$$




$$
{ }^{7} \mathrm{Li}+\mathrm{p} \rightarrow 2^{4} \mathrm{He}+17.347 \mathrm{MeV} .
$$

The $p p I I I$ chain starts with the production of a ${ }^{8} \mathrm{~B}$ nucleus, and is the source of neutrinos that have a maximum energy of $15.04 \mathrm{MeV}$ :

$$
\begin{gathered}
{ }^{7} \mathrm{Be}+\mathrm{p} \rightarrow{ }^{8} \mathrm{~B}+\gamma+0.137 \mathrm{MeV} \\
{ }^{8} \mathrm{~B} \rightarrow{ }^{8} \mathrm{Be}^{*}+\mathrm{e}^{+}+\nu_{\mathrm{e}}+15.04 \mathrm{MeV} \\
{ }^{8} \mathrm{Be}^{*} \rightarrow 2^{4} \mathrm{He}+3.030 \mathrm{MeV}
\end{gathered}
$$

Even if it occurs with a small branching fraction, the $p p$ III chain is important for experimental solar neutrino physics because of the production of neutrinos being more energetic than those produced by other $p p$ reactions. The ${ }^{8} \mathrm{~B}$ neutrinos, for example, are an excellent probe of the Sun as they are the main neutrino signal detected at the Sudbury Neutrino Observatory. Hep neutrinos have even higher energies, but they are very rare and thus challenging to study. However, their detection would serve as an important confirmation of our complete understanding of the energy production processes inside the Sun.

The CNO cycle as a source of solar energy was proposed by Bethe [35] in the late 1930 's. Less then $2 \%$ of the solar energy is produced by the CNO cycle as it is plausible only in the presence of heavier elements. This chain is interesting for stelar astrophysics and evolution, since it is expected that in certain conditions it dominates the $p p$ chain. For the solar composition, it exceeds the $p p$ chain at temperatures higher than $1.8 \times 10^{7} \mathrm{~K}[36]$. The main CNO cycle (at the $99.9 \%$ ) proceeds via the following interactions: 


$$
\begin{gathered}
{ }^{12} \mathrm{C}+\mathrm{p} \rightarrow{ }^{13} \mathrm{~N}+\gamma, \\
{ }^{13} \mathrm{~N} \rightarrow{ }^{13} \mathrm{C}+\mathrm{e}^{+}+\nu_{\mathrm{e}}+1.119 \mathrm{MeV}, \\
{ }^{13} \mathrm{C}+\mathrm{p} \rightarrow{ }^{14} \mathrm{~N}+\gamma, \\
{ }^{14} \mathrm{~N}+\mathrm{p} \rightarrow{ }^{15} \mathrm{O}+\gamma, \\
{ }^{15} \mathrm{O} \rightarrow{ }^{15} \mathrm{~N}+\mathrm{e}^{+}+\nu_{\mathrm{e}}+1.732 \mathrm{MeV}, \\
{ }^{15} \mathrm{~N}+\mathrm{p} \rightarrow{ }^{12} \mathrm{C}+{ }^{4} \mathrm{He} .
\end{gathered}
$$

A carbon nucleus from the process of Equation (3.18) re-initiate the reaction given in Equation (3.13), thus closing the chain. The other CNO subchain of reactions (at the $0.1 \%$ level) starts by following the reaction of Equation (3.17):

$$
\begin{gathered}
{ }^{15} \mathrm{~N}+\mathrm{p} \rightarrow{ }^{16} \mathrm{O}+\gamma, \\
{ }^{16} \mathrm{O}+\mathrm{p} \rightarrow{ }^{17} \mathrm{~F}+\gamma, \\
{ }^{17} \mathrm{~F} \rightarrow{ }^{17} \mathrm{O}+\mathrm{e}^{+}+\nu_{\mathrm{e}}+1.740 \mathrm{MeV}, \\
{ }^{17} \mathrm{O}+\mathrm{p} \rightarrow{ }^{14} \mathrm{~N}+{ }^{4} \mathrm{He} .
\end{gathered}
$$

A nitrogen nucleus from the last reaction initiate the process shown in Equation (3.16), thus closing the chain with the subsequent reaction of Equation (3.17).

With the knowledge of the solar neutrino production mechanism, we are interested in the predictions of the total solar neutrino output and its flux at the Earth, since it can be measured experimentally. The solar neutrino fluxes can be 
predicted only by solving the evolution equations of the Sun, which is achieved by the construction of the Standard Solar Model (SSM).

\subsection{The Standard Solar Model}

The most fundamental understanding of a stationary star like our Sun comes from the hydrostatic equilibrium equation that describes a balance of the stelar gravitational collapse by its radiative and mechanical pressure gradients $d P / d r$ :

$$
\frac{d P}{d r}=-\frac{G m(r) \rho}{r^{2}}
$$

where $G$ is the gravitational constant, $m(r)$ is the mass within the radius $r$ related with the density $\rho$ as:

$$
\frac{d m(r)}{d r}=4 \pi r^{2} \rho .
$$

Another important evolution equation for a star is the energy conservation relation that links the luminosity output $L$ from a spherical shell at the radius $r$ with the total energy produced by nuclear reactions and energy losses. For the star in hydrostatic equilibrium, the energy from nuclear fusion is given by the power per unit mass $\epsilon_{n}$, while the energy loss due to the emission of neutrinos is given by the luminosity per unit mass $\epsilon_{\nu}$ :

$$
\frac{d L(r)}{d r}=4 \pi r^{2} \rho\left(\epsilon_{n}-\epsilon_{\nu}\right)
$$

The Standard Solar Model solves Equations (3.23) and (3.24) for the Sun, by specifying its equation of state, $P=P(T, \rho)$, under standard assumptions 
for stars, and by including additional equations for the energy transport trough the solar interior via convective and radiative processes. The calculation starts with the initial conditions for the homogenous solar composition at time $t=0$ and solves the evolution equations for the present time $t=4.6 \times 10^{9}$ years. The astrophysical input data needed to infer the properties of the Sun at the present time are: the surface abundances of heavier elements in the solar atmosphere, the diffusion factors that account for the difference in the element abundances between the interior and the solar atmosphere, the nuclear cross-section factors, the Sun's luminosity $L_{\odot}$ which is used as a constraint for the predicted luminosity by the model, the opacity and its dependancy on the chemical composition, and the solar age [37-40].

The predictions of the SSM are thoroughly tested with the observational data from helioseismology. The helioseismological experiments measure pressure and $p$-mode oscillations of the Sun, from which both the speed of sound and matter density in the Sun can be inferred. These measurements ruled out many solar models with non-standard properties of the Sun. Solar sound speed predictions from the most recent SSM constructed by John Bachall and his collaborators [38-40] show excellent agreement with helioseismology data. The predictions on distributions of the solar sound speeds and densities from the SSM in Reference [40] agree with the helioseismological data on average at the $0.1 \%$ and the $1.5 \%$ level, respectively. This is quite an achievement considering the complexity of the Sun.

In order to study physics of solar neutrinos, we are interested in the distributions of electron and nucleon scatterers in the solar interior and the neutrino production regions, which are used in the calculation of neutrino survival probability $P_{\nu_{\alpha} \rightarrow \nu_{\alpha}}$ of Equation (2.25). The other, not less important, requirement for 
our analyses is the precise determination of the solar neutrino fluxes. The spectra of solar neutrinos fluxes from the $p p$ and $\mathrm{CNO}$ chains, which are obtained from the latest work of Bachall et al. [40], are depicted in Figure 3.1. For the purpose of

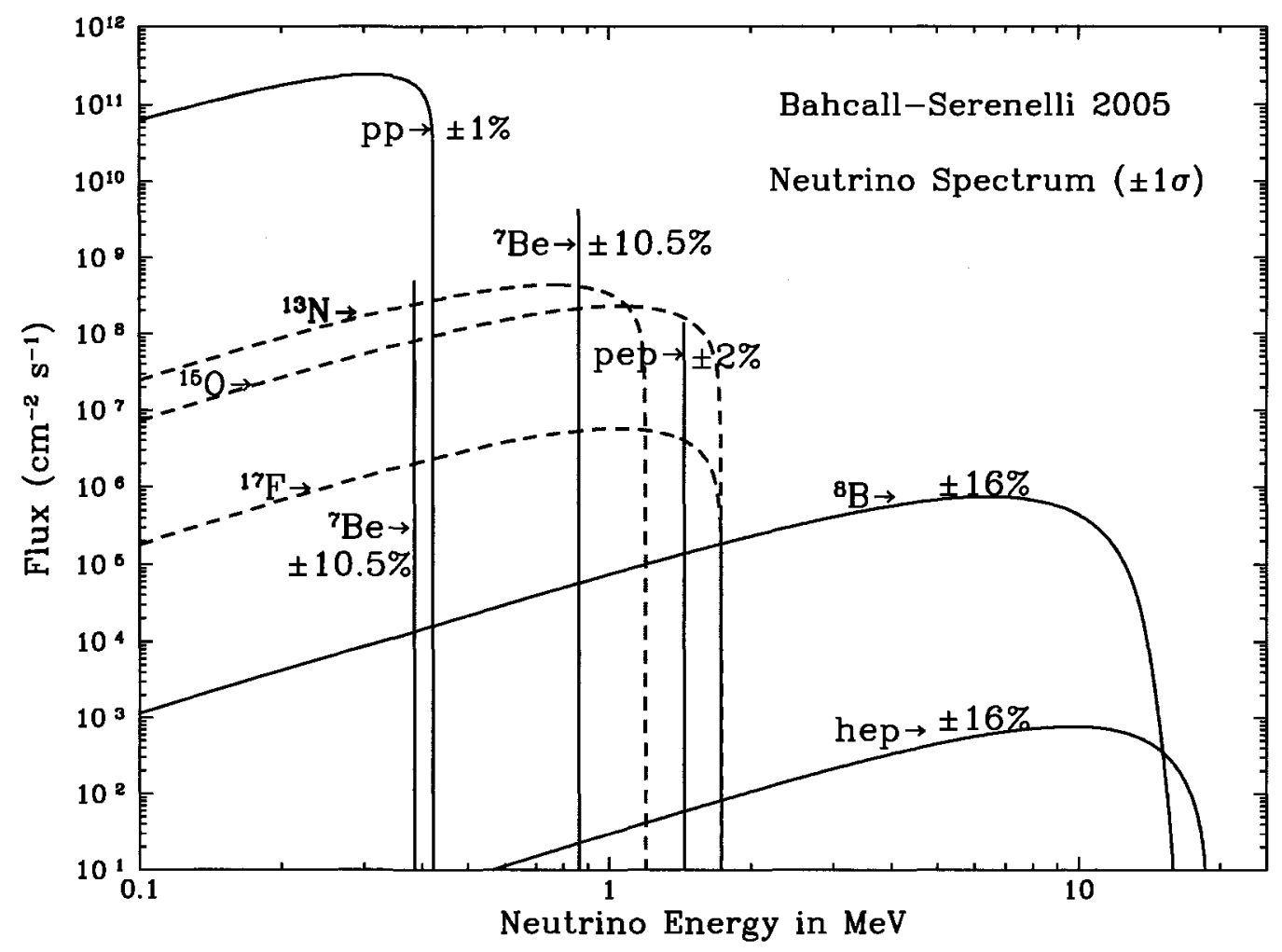

Figure 3.1: Energy spectra of neutrino fluxes from the $p p$ and CNO chains from the latest SSM. This figure is taken from Reference [43].

the analyses presented in this dissertation, the Standard Solar Models BP00 from Reference [38], BP04 from Reference [39] and BP05(OP) from Reference [40] are used. The comparison of the neutrino fluxes from eight different solar neutrino sources are tabulated in Table 3.1 . 
Table 3.1: Neutrino fluxes from BP00 [38], BP04 [39], and BS05(OP) [40] for the principal fusion reactions.

\begin{tabular}{lccc}
\hline \hline $\begin{array}{l}\text { Neutrino } \\
\text { source }\end{array}$ & BP00 & Neutrino flux in cm $\mathrm{cm}^{-2} \mathrm{~s}^{-1}$ \\
\hline$p p$ & $(5.95 \pm 0.01) \times 10^{10}$ & $(5.94 \pm 0.01) \times 10^{10}$ & $(5.99 \pm 0.009) \times 10^{10}$ \\
pep & $(1.40 \pm 0.015) \times 10^{8}$ & $(1.40 \pm 0.02) \times 10^{8}$ & $(1.42 \pm 0.015) \times 10^{8}$ \\
$h e p$ & $9.3 \times 10^{3}$ & $(7.88 \pm 0.16) \times 10^{3}$ & $(7.93 \pm 0.115) \times 10^{3}$ \\
${ }^{7} \mathrm{Be}$ & $(4.77 \pm 0.10) \times 10^{9}$ & $(4.86 \pm 0.12) \times 10^{9}$ & $(4.84 \pm 0.105) \times 10^{9}$ \\
${ }^{8} \mathrm{~B}$ & $\left(5.05_{-0.16}^{+0.20}\right) \times 10^{6}$ & $(5.79 \pm 0.23) \times 10^{6}$ & $\left(5.69_{-0.147}^{+0.173}\right) \times 10^{6}$ \\
${ }^{13} \mathrm{~N}$ & $\left(5.48_{-0.17}^{+0.21}\right) \times 10^{8}$ & $\left(5.71_{-0.35}^{+0.37}\right) \times 10^{8}$ & $\left(3.07_{-0.278}^{+0.309}\right) \times 10^{8}$ \\
${ }^{15} \mathrm{O}$ & $\left(4.80_{-0.19}^{+0.25}\right) \times 10^{8}$ & $\left(5.03_{-0.39}^{+0.43}\right) \times 10^{8}$ & $\left(2.31_{-0.284}^{+0.328}\right) \times 10^{8}$ \\
${ }^{17} \mathrm{~F}$ & $(5.63 \pm 0.25) \times 10^{6}$ & $(5.91 \pm 0.44) \times 10^{6}$ & $(5.83 \pm 0.50) \times 10^{6}$ \\
\hline \hline
\end{tabular}

\subsection{Solar Neutrino Problem}

The pioneering experiment for the detection of solar neutrinos was the radiochemical Homestake detector constructed by Raymond Davis and his collaborators in 1967. Homestake is located at the depth of $1478 \mathrm{~m}$ (or $4200 \mathrm{~m}$ of water equivalent) in the Homestake Gold Mine at Lead, South Dakota, USA. The detection medium at Homestake was ${ }^{37} \mathrm{Cl}$ filled into the detector tank in the form 615 tonnes of tetrachloroethylene $\mathrm{C}_{2} \mathrm{Cl}_{4}$. Solar neutrinos were detected through the inverse $\beta$-decay reaction of chlorine into argon:

$$
\nu_{e}+{ }^{37} \mathrm{Cl} \rightarrow{ }^{37} \mathrm{Ar}+e^{-},
$$

with the neutrino energy threshold of $E_{\nu}^{\text {th }}=0.814 \mathrm{MeV}$, meaning that this experiment was sensitive to all neutrinos except the $p p$ and low energy line of the ${ }^{7} \mathrm{Be}$ neutrinos at $0.387 \mathrm{MeV}$, as depicted in Figure 3.1.

In 1968, the first results from Homestake [41] with the solar neutrino flux 
measurement of less than 3 solar neutrino unit $(\mathrm{SNU})^{1}$ indicated that there was a discrepancy between the data and the expectation of about 7.5 SNU from the SSM [42]. This discrepancy was the origin of the so-called Solar Neutrino Problem (SNP). After more that 30 years of data collection, the final solar neutrino rate measured by Homestake is [9]:

$$
R_{\mathrm{Cl}}=[2.45 \pm 0.16(\text { stat }) \pm 0.16(\text { syst })] \mathrm{SNU},
$$

which is approximately $3.3 \sigma$ away from the SSM BP04 expectation of $(8.50 \pm 1.25)$ SNU [43]. The SNP, which was reported later by other experiments as well, raised a question whether the SSM calculations are wrong or our understanding of neutrinos is incomplete. Considering the phenomenal agreement between the SSM, helioseismology and the Sun's luminosity, nowadays, the most probable explanation for the SNP is given by the neutrino mixing hypothesis and MSW effect presented in Chapter 2. Matter induced neutrino mixing leads to the effects of flavour transition of electron neutrinos while they travel through the Sun, oscillations in the vacuum and finally, plausible regeneration of electron neutrinos traversing the core of the Earth.

The Solar Neutrino Problem was identified by the international scientific community as a fundamental open question in particle astrophysics. It then initiated the commissioning of several experimental programs to measure the solar neutrino fluxes. Each experiment used different and complementary detection techniques to sample different neutrino interactions in order to constrain the Standard Solar Model.

\footnotetext{
${ }^{1}$ One SNU is equivalent to $10^{-36}$ neutrino captures per target atom per second.
} 
There were three radiochemical experiments that used gallium as a solar neutrino target through this reaction:

$$
\nu_{e}+{ }^{71} \mathrm{Ga} \rightarrow{ }^{71} \mathrm{Ge}+e^{-},
$$

with a neutrino energy threshold of $E_{\nu}^{\text {th }}=0.223 \mathrm{MeV}$. Therefore they were able to detect solar neutrinos from all eight sources described in the previous section. The first of these experiments was the Gallium Experiment (Gallex), which was located in the Laboratori Nazionali del Gran Gasso, Italy, at the depth of $3300 \mathrm{~m}$ of water equivalent. It was filled with approximately 100 tonnes of liquid gallium chloride, and collected data from 1991 to 1997. The same detector was upgraded and operated as the Gallium Neutrino Observatory (GNO) between 1998 and 2003. The total combined rate measured by these two experiments is [10]:

$$
R_{\mathrm{Ga}}^{\mathrm{Gallex} / \mathrm{GNO}}=[69.3 \pm 4.1(\text { stat }) \pm 3.6(\text { syst })] \mathrm{SNU},
$$

which disagrees with the SSM expectation of $130.54_{-7.53}^{+9.54} \mathrm{SNU}$ at more than $5 \sigma$. The third gallium experiment is the Soviet-American Gallium Experiment (SAGE) situated in the Baksan Neutrino Observatory (BNO) in the Russian Caucasus mountains at the depth of approximately $4700 \mathrm{~m}$ of water equivalent. The latest results from SAGE [44] reported a total neutrino rate of:

$$
R_{\mathrm{Ga}}^{\mathrm{SAGE}}=[66.9 \pm 3.9(\text { stat }) \pm 3.6(\text { syst })] \mathrm{SNU} .
$$

It disagrees with the SSM prediction at the $5 \sigma$ level.

The comparison of the SSM BS05(OP) predictions for the solar neutrino rates 
and fluxes to the measurements from the radiochemical experiments $(\mathrm{Ga}$ and $\mathrm{Cl})$ are summarized in Figure 3.2.

\section{Total Rates: Standard Model vs. Experiment Bahcall-Serenelli 2005 [BS05(OP)]}

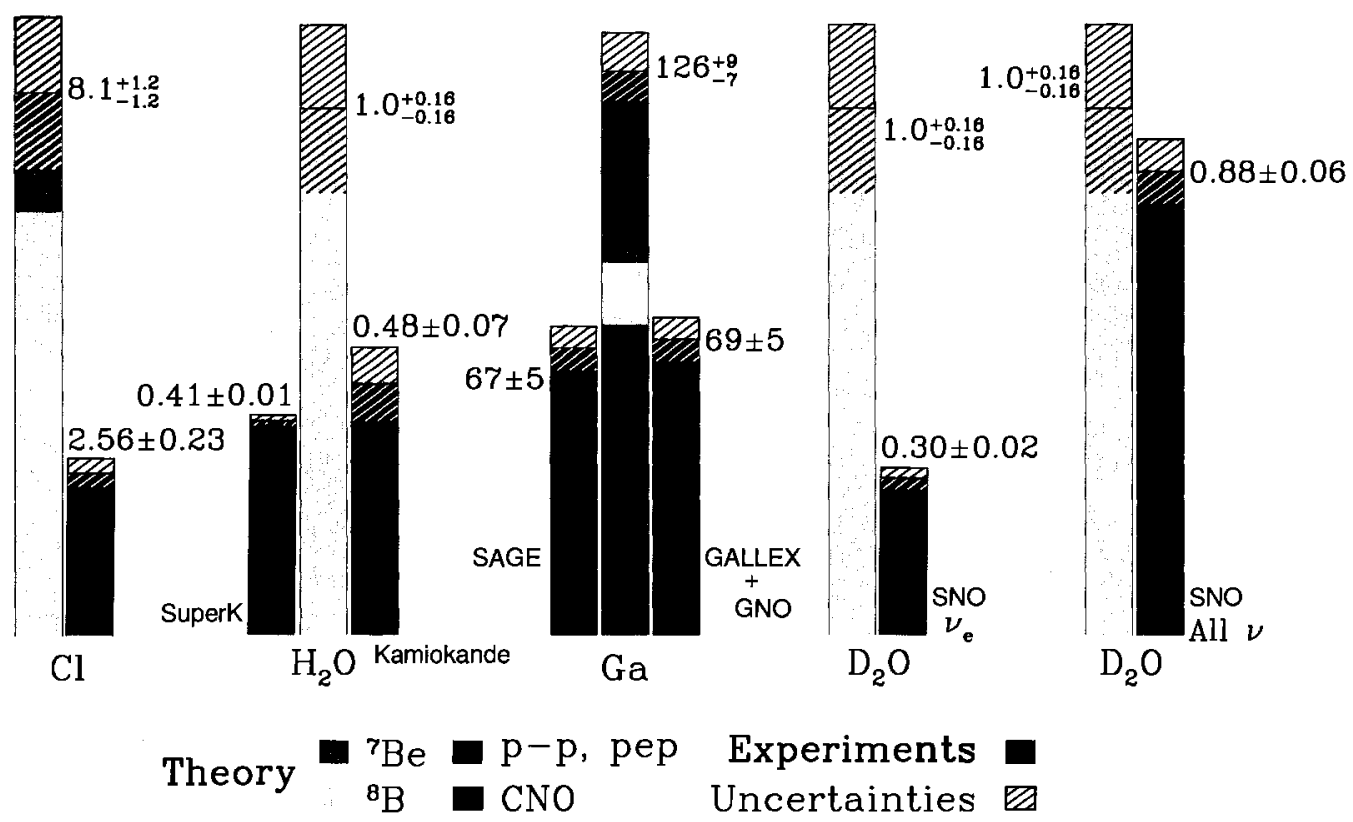

Figure 3.2: Comparison of the SSM BS05(OP) predictions for the neutrino rates and fluxes to the results reported by solar neutrino experiments. This figure is taken from Reference [43].

In addition to the radiochemical rate experiments, there are several real-time solar neutrino experiments that collected information on neutrino interactions with the Cerenkov light emitted by a signature of interaction, which is a charged particle moving with the speed that is greater than the speed of light in the medium of interest. 
The Kamiokande experiment was the first real-time water based Čerenkov detector. The experiment operated between 1987 and 1995. It measured a flux of the ${ }^{8} \mathrm{~B}$ neutrinos of [12]:

$$
\phi_{8 \mathrm{~B}}^{\mathrm{K}}=[2.80 \pm 0.19(\text { stat }) \pm 0.33(\text { syst })] \times 10^{6} \mathrm{~cm}^{-2} \mathrm{~s}^{-1} .
$$

The Super-Kamiokande (SK) experiment is a Čerenkov detector that uses a $50 \mathrm{kton}$ of light water as a detection medium for solar neutrinos. The detector is located approximately $1000 \mathrm{~m}$ underground, or $2600 \mathrm{~m}$ of water equivalent, in the Kamioka mine, Japan. This experiment is the successor of the 3000 ton Kamiokande experiment and its results superseded Kamiokande's early results. SK detects solar neutrinos from the ${ }^{8} \mathrm{~B}$ and hep sources via elastic scattering process on electrons:

$$
\nu_{\alpha}+e^{-} \rightarrow \nu_{\alpha}+e^{-}
$$

After the first phase of operation, which is known as SK-I phase and lasted between April 1996 and July 2001, the measured flux of the ${ }^{8} \mathrm{~B}$ neutrinos was recently updated and yields [13]:

$$
\phi_{8_{\mathrm{B}}}^{\mathrm{SK}-\mathrm{I}}=[2.35 \pm 0.02 \text { (stat) } \pm 0.08(\text { syst })] \times 10^{6} \mathrm{~cm}^{-2} \mathrm{~s}^{-1} .
$$

This measurement disagrees with the SSM prediction of $(5.79 \pm 0.23) \times 10^{6} \mathrm{~cm}^{-2} \mathrm{~S}^{-1}$ at more than $2.5 \sigma$. The SK placed an upper limit on the hep neutrinos reporting $\phi_{h e p}^{\mathrm{SK}-\mathrm{I}}<73 \times 10^{3} \mathrm{~cm}^{-2} \mathrm{~s}^{-1}$ at $90 \% \mathrm{CL}$. However, a much better limit on the hep flux is achieved by the SNO collaboration [45]: $\phi_{h e p}^{\text {SNO }}<23 \times 10^{3} \mathrm{~cm}^{-2} \mathrm{~s}^{-1}$ at $90 \%$ CL. The second phase of Super-Kamiokande (SK-II) was operated from January 
2003 to February 2004 [46], but these data were not ready to be analyzed in this thesis. The analysis of the SK-II data is in the area of interest for our future work. The SK-I result is also depicted in Figure 3.2.

The last solar experiment whose measurement contribute to our analyses is the Borexino experiment. Borexino is a low background scintillator detector situated at the Laboratori Nazionali del Gran Sasso. It is the first real-time detector capable of measuring low energy solar neutrinos, in particular the $0.862 \mathrm{MeV}$ line of the ${ }^{7}$ Be neutrinos. Borexino detects solar neutrinos via their elastic scattering interaction with the electrons in a scintillator medium composed of pseudocumene. The latest measurement from Borexino [47] reports the rate of the $0.862 \mathrm{MeV}^{7} \mathrm{Be}$ neutrino line as:

$$
R_{7_{\mathrm{Be}}}^{\text {Borex. }}=[49 \pm 3(\text { stat }) \pm 4(\text { syst })] \text { counts per day per } 100 \text { ton }
$$

which should be compared to the SSM expectation of $R_{7 \mathrm{Be}}^{\mathrm{SSM}}=(75 \pm 4)$ counts per day per 100 ton.

\subsection{Finally a Solution}

We already implied that the most probable solution to the Solar Neutrino Problem is the effect of neutrino flavour conversion from the neutrino mixing hypothesis. The first experimental confirmation of both the SSM predictions and the solar neutrino flavour transformation came from the Sudbury Neutrino Observatory (SNO) in April 2002 [14]. SNO measured the electron component of the solar 
neutrino flux from the ${ }^{8} \mathrm{~B}$ source as:

$$
\phi_{\nu_{e}}=1.76_{-0.05}^{+0.05}(\text { stat })_{-0.09}^{+0.09}(\text { syst }) \times 10^{6} \mathrm{~cm}^{-2} \mathrm{~s}^{-1},
$$

and non-electron component of the solar neutrino flux of:

$$
\phi_{\nu_{\mu, \tau}}=3.41_{-0.45}^{+0.45}(\text { stat })_{-0.45}^{+0.48}(\text { syst }) \times 10^{6} \mathrm{~cm}^{-2} \mathrm{~s}^{-1},
$$

which disagrees with zero non-electron neutrino component of the flux at $5.3 \sigma$. The total measured solar neutrino flux, composed of all active neutrino flavours is:

$$
\phi_{8_{\mathrm{B}}}=5.09_{-0.43}^{+0.44}(\text { stat })_{-0.43}^{+0.46}(\text { syst }) \times 10^{6} \mathrm{~cm}^{-2} \mathrm{~s}^{-1}
$$

which confirmed the SSM predictions and solved the Solar Neutrino Problem. This result was acclaimed as one of the main scientific achievements in recent years.

From the April 2002 milestone of SNO, the field of neutrino physics shifted from solving the SNP to the precise determination of the matter-induced oscillation parameters of solar neutrinos. This coincidence with the effort of the author of this thesis to join the SNO collaboration in 2002.

Detailed descriptions of the Sudbury Neutrino Observatory experiment and its data samples are presented in the next three chapters. In Chapters 7 and 8 , more detailed analyses of the SNO data and the associated physics interpretation will be presented. 


\section{Chapter 4}

\section{Experimental Apparatus}

The Sudbury Neutrino Observatory (SNO) is a real-time heavy water Cerenkov detector situated two kilometers underground in the Inco Ltd., Creighton mine near Sudbury, Ontario, Canada. The experiment is built to study fundamental properties of solar neutrinos, primarily their mixing parameters: $\tan ^{2} \theta$ and $\Delta m^{2}$. The uniqueness of SNO resides in its capability to measure the total active solar neutrino flux via neutral-current $(\mathrm{NC})$ interaction, in addition to its measurements of the total flux of $\nu_{e}$ via charged-current (CC) interaction. By using NC flux measurements, SNO is able to directly test both the SSM predictions on the total ${ }^{8} \mathrm{~B}$ neutrino flux and the neutrino oscillation hypothesis. The properties of the SNO detector, as well as a description of neutrino interactions occurring in its target medium, are presented in this chapter. 


\subsection{The Sudbury Neutrino Observatory}

The center of the SNO detector is located $2092 \mathrm{~m}$ underground or approximately $6000 \mathrm{~m}$ of water equivalent, at latitude and longitude of $46^{\circ} 28^{\prime} 30^{\prime \prime} \mathrm{N}$ and $81^{\circ} 12^{\prime} 04^{\prime \prime}$ W in the Inco Ltd., Creighton mine near Sudbury. At this depths, the detector is well shielded from the cosmic rays; in particular only about 70 muons per day traverse its interior. The detector is filled with 1000 tonnes of $99.92 \%$ isotopically pure heavy water $\left(D_{2} \mathrm{O}\right)$, which serves as neutrino detection medium, contained inside an acrylic sphere of $12 \mathrm{~m}$ in diameter. The acrylic vessel (AV) is suspended by ten loops of rope. Access to the $\mathrm{D}_{2} \mathrm{O}$ volume for calibration and installation of NCD strings is provided by a $1.5 \mathrm{~m}$ diameter by $6.8 \mathrm{~m}$ tall neck. The AV sphere is surrounded by a geodesic structure of $18 \mathrm{~m}$ in diameter, which carries an array of 9456 inward-looking $20 \mathrm{~cm}$ photomultiplier tubes (PMTs) that detect Cerenkov radiation originated from the interactions inside the $\mathrm{D}_{2} \mathrm{O}$ volume. The primary design goal for the PMTs is to provide high photon detection efficiency and excellent timing resolution (RMS $=1.7 \mathrm{nsec}$ ), and to contain a minimal amount of radioactivity. A concentrator of light is mounted on each PMT to increase the photocathode coverage of the entire array to $54 \%$ of solid angle from the central axis of the detector. The geodesic structure and acrylic vessel are immersed in a barrel-shaped cavity, $22 \mathrm{~m}$ in diameter and $24 \mathrm{~m}$ in height, filled with 7400 tonnes of ultra pure light water $\left(\mathrm{H}_{2} \mathrm{O}\right)$, which provides shielding from the radioactivity coming from the rocks surrounding the detector. Additional PMTs are installed to serve as a background vetoes. A total of 91 outward-looking PMTs are mounted on the geodesic sphere and 23 inward-looking PMTs are arranged at the top of the $\mathrm{H}_{2} \mathrm{O}$ volume to trigger on cosmic rays. An extra four PMTs 
in the neck region are installed to count possible instrumentation backgrounds. Fourteen magnetic compensation coils are mounted on the detector in order to cancel the vertical component of the Earth's magnetic field and thus to increase the detection efficiency of the PMT array by approximately $10 \%$. A schematic view of the detector is shown in Figure 4.1.

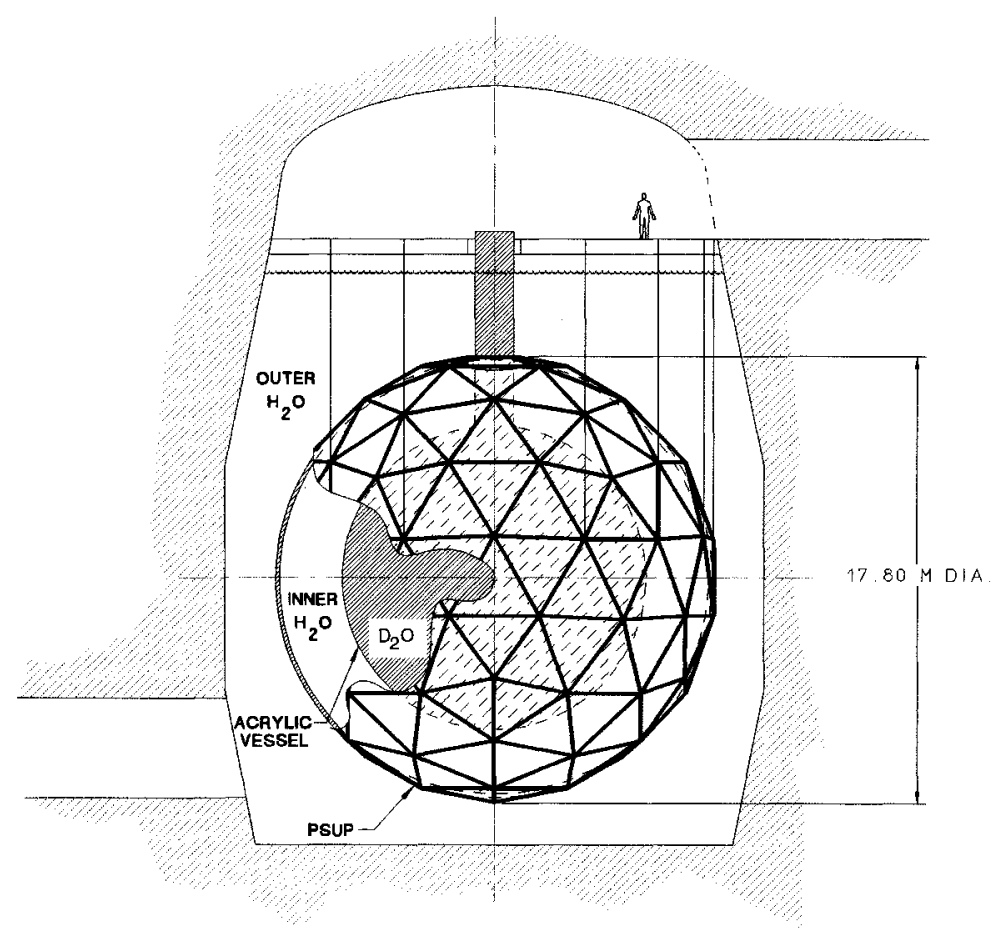

Figure 4.1: A schematic of the SNO detector. This figure is taken from Reference [49].

During the last phase of the experiment's operation an array of ${ }^{3} \mathrm{He}$ filled proportional counters, called Neutral Current Detection or NCD array, was deployed in order to re-measure the total flux of solar neutrinos from the NC interaction in 
the $\mathrm{D}_{2} \mathrm{O}$ by a different method independent from its previous measurements with the PMT array. The NCD array consists of $36{ }^{3} \mathrm{He}$ strings, which are immersed in the $\mathrm{D}_{2} \mathrm{O}$ in order to detect neutrons from the $\mathrm{NC}$ interaction. An additional four counters filled with ${ }^{4} \mathrm{He}$, thus being insensitive to neutrons, are deployed to study background events, primarily $\alpha$ particles.

A low level of radioactivity is preserved inside the heavy water; this is crucial for a precise measurement of the neutrino signal at SNO. The radioactive ${ }^{208} \mathrm{Tl}$ and ${ }^{214} \mathrm{Bi}$ produced from the decay chains of ${ }^{232} \mathrm{Th}$ and ${ }^{238} \mathrm{U}$ emit $\gamma$ rays above the energy threshold for the photo-disintegration of the deuteron $(2.22 \mathrm{MeV})$. The neutrons produced from these processes mimic the signal from the $\mathrm{NC}$ neutrino interactions, in particular one background neutron is expected from a concentration of $3.8 \times 10^{-15} \mathrm{~g} \mathrm{Th} / \mathrm{g}_{2} \mathrm{O}$ or $30 \times 10^{-15} \mathrm{~g} \mathrm{U} / \mathrm{g} \mathrm{D}_{2} \mathrm{O}$ [48]. The radioactive contamination inside the heavy water target is preserved far bellow these values (by more than $70 \%$ ), assuring the rate of the photo-disintegration neutrons to be less than $3-4 \%$ of the expected rate from the $\mathrm{NC}$ signal.

Further information concerning the detector properties can be found in Reference [49].

\subsection{Signature of Neutrinos and Interactions}

The heavy water in the SNO detector serves primary as a target medium for the detection of neutrinos coming from the Sun. However, the heavy water target could also serve as a detection medium for neutrinos from supernova explosion, diffuse supernova neutrino background, and atmospheric events as well as antineutrinos from the Sun and other sources. 
Events in SNO are triggered by Čerenkov radiation produced by ultra-relativistic charged particles. There are three types of electroweak interaction that occur in the $\mathrm{D}_{2} \mathrm{O}$ when the volume is traversed by a beam of neutrinos: the neutral-current $(\mathrm{NC})$, the charged-current $(\mathrm{CC})$ and the elastic scattering (ES) interactions.

\subsection{1 Čerenkov Radiation}

The heavy water of SNO allows for real time detection of neutrinos by observing the ultra-relativistic charged leptons, that are produced by neutrino interactions.

When a charged particle propagates through a medium, which has index of refraction $n$, with a velocity $v$ greater than the phase velocity of light $v_{\mathrm{ph}}=c / n$ in that medium, it emits Cerenkov radiation. The number of photons $N$ emitted from Čerenkov radiation is given by:

$$
\frac{d^{2} N}{d x d \lambda}=\frac{2 \pi \alpha Z^{2}}{\lambda^{2}}\left(1-\frac{c^{2}}{n^{2} v^{2}}\right)
$$

where $x$ is the distance travelled by the charged particle, $\lambda$ is the wavelength of the emitted photon, $\alpha \sim 1 / 137$ is the fine structure constant and $Z$ is the charge number. The Čerenkov radiation is emitted in a cone with a characteristic angle $\cos \theta_{\check{\mathrm{C}}}=c / n v$.

In $\mathrm{D}_{2} \mathrm{O}$, an electron is usually produced as a result of neutrino interactions. The threshold for an electron to produce Čerenkov radiation in $\mathrm{D}_{2} \mathrm{O}(\mathrm{n}=1.34)$ is approximately $0.767 \mathrm{MeV}$ of total energy. This is far bellow the kinematic energy threshold used for the analyses at SNO. The wavelengths of the Čerenkov photons that are emitted by the electron in the energy window used for data analyses are in the 200 to $600 \mathrm{~nm}$ range. This range of wavelengths is appropriate for detection 
by PMTs.

\subsubsection{The Charged-Current Interaction}

The charged-current interaction involves the exchange of a charged $\mathrm{W}^{ \pm}$boson associated with the neutral and charged leptons of the same flavour. At SNO, this reaction is exclusive to the electron neutrinos only because solar neutrinos of other types do not have enough energy to produce much heavier muon or tau leptons. The electron neutrino weakly interacts with the down quark of a neutron bound in the deuteron and exchanges a $\mathrm{W}^{+}$boson. As a result of this interaction, the down quark is transformed into an up quark and deuteron is broken down into the two protons, while the electron neutrino is transformed into an electron:

$$
\nu_{e}+d \rightarrow p+p+e^{-}
$$

The threshold for CC reaction at SNO is $E_{\nu}^{\mathrm{th}}=2 m_{p}+m_{e}-m_{d}=1.442 \mathrm{MeV}$. However SNO has a higher analyses thresholds of $5.5-6.5 \mathrm{MeV}$ due to the high rate of backgrounds at lower energies. The thresholds in this range correspond to neutrino energy thresholds of $6.9-7.9 \mathrm{MeV}$, hence SNO is sensitive only to the ${ }^{8} \mathrm{~B}$ and rare hep solar neutrinos.

The resulting electron from the CC interaction has an energy highly correlated with the energy of the incident electron neutrino, thus providing an important information on the shape of the ${ }^{8} \mathrm{~B}$ neutrino energy spectrum. This electron moves faster then light in $\mathrm{D}_{2} \mathrm{O}$, therefore it emits a Čerenkov light, that is detected by the PMT array of the detector. An electron produced by the CC reaction does not carry a significant directional information on the incident neutrino because it 
is emitted with an angular distribution of the form [50]:

$$
f\left(\theta_{\odot}\right)=1-0.340 \cos \theta_{\odot}
$$

where $\theta_{\odot}$ is the angle between the direction of the Sun and direction of the emitted electron.

\subsubsection{The Neutral-Current Interaction}

The neutral-current interaction is unique to the SNO detector. Through the NC reaction all active neutrino flavours are detected with the same sensitivity: $\nu_{e}, \nu_{\mu}$ and $\nu_{\tau}$. Hence it allows for determination of the total ${ }^{8} \mathrm{~B}$ neutrino flux. With its neutral-current measurements SNO directly tested and confirmed the prediction of the SSM $[14,48,51]$. By comparing the CC and NC flux measurements, SNO proves that the solar neutrino problem is due to neutrino mixing and flavour transitions.

The neutral-current interaction is mediated by a neutral $Z^{0}$ boson that equally couples to all three active neutrino flavours $\nu_{\alpha}$, where $\alpha=e, \mu$ or $\tau$. The NC interaction of a neutrino $\nu_{\alpha}$ on deuterium disintegrates the deuteron into a neutron and a proton:

$$
\nu_{\alpha}+d \rightarrow n+p+\nu_{\alpha},
$$

with the neutrino energy threshold of $E_{\nu}^{\text {th }}=m_{n}+m_{p}-m_{d}=2.224 \mathrm{MeV}$.

A detection of the final neutron from this reaction is used by SNO as the signature for the NC interaction. The operation of SNO experiment is divided in three phases with respect to various neutron detection techniques. 
SNO-I phase or $\mathrm{D}_{2} \mathrm{O}$ phase In this phase, SNO took data between November $2^{\text {nd }}, 1999$ to May $28^{\text {th }}, 2001$ with 306.4 live days in total, pure $\mathrm{D}_{2} \mathrm{O}$ was used to observe neutron capture on deuterium:

$$
n+d \rightarrow{ }^{3} \mathrm{H}+\gamma
$$

The signature of this neutron capture reaction is a $6.25 \mathrm{MeV} \gamma$-ray that has sufficient energy to Compton scatter electrons, that can subsequently be reconstructed with the detection of their Cerenkov radiation. The crosssection for this neutron capture reaction is approximately $0.5 \mathrm{mb}$.

SNO-II phase or salt phase For the second phase of operation, which took place between July $26^{\text {th }}, 2001$ to August $28^{\text {th }}, 2003$ with 391.4 live days in total, about 2 tons of $\mathrm{NaCl}$ brine were added to the $\mathrm{D}_{2} \mathrm{O}$ in order to increase the neutron detection efficiency with the reaction:

$$
n+{ }^{35} \mathrm{Cl} \rightarrow{ }^{36} \mathrm{Cl}+\gamma^{\prime} s
$$

From the neutron capture on ${ }^{35} \mathrm{Cl}$, multiple $\gamma$-rays (average multiplicity of about three) with a total energy of $8.57 \mathrm{MeV}$ are produced. The crosssection for neutron capture on chlorine is $44 \mathrm{~b}$. This is much higher than neutron capture on deuteron and therefore increased the SNO neutron detection efficiency. Almost $80 \%$ of NC neutrons were captured on chlorine. The Compton scattered electrons produced by the multiply $\gamma$-rays are distributed more isotropically than single electrons from the CC interactions. The isotropy of Čerenkov radiation emitted during a neutrino event provided 
a powerful tool for separation of $\mathrm{NC}$ from $\mathrm{CC}$ signal at SNO. In addition, the higher total energy released during the neutron capture on chlorine allowed SNO to make a precise measurement of $\mathrm{NC}$ signal well above the region contaminated by low energy background events.

SNO-III phase or NCD phase For its final phase, SNO operated between November $27^{\text {th }}, 2004$ to November $28^{\text {th }}, 2006$ with 385.2 live days in total. During this phase, the ${ }^{3} \mathrm{He}$ proportional counters served as the main detection tool for the neutrons produced by $\mathrm{NC}$ interaction, through the following reaction:

$$
n+{ }^{3} \mathrm{He} \rightarrow p+{ }^{3} \mathrm{H}+0.764 \mathrm{MeV} .
$$

The cross-section for this reaction is enormous: $5330 \mathrm{~b}$, meaning that most of the neutrons are detected by the NCD counters via triton-proton pairs producing the ionization tracks and causing an electrical pulse on the anode wire of the proportional counters. This method of neutron detection is well uncorrelated with the previous neutral-current measurements from SNO, therefore it provides an independent test of both the SSM prediction and neutrino oscillation hypothesis by the same experiment.

\subsubsection{The Elastic Scattering Interaction}

The elastic scattering of neutrino on atomic electron is mediated by a $W^{-}$boson if the incident neutrino is of electron flavour and by a $Z^{0}$ boson for all three active neutrino flavours:

$$
\nu_{\alpha}+e^{-} \rightarrow \nu_{\alpha}+e^{-} .
$$


This reaction is mainly sensitive to electron neutrinos because their scattering cross-section is approximately 6.5 times higher than that of other neutrino flavours. The scattered electron moves into a direction that is highly correlated with the position of the Sun, which allows for separation of the ES signal in the detector by using the information on the angle $\left(\cos \theta_{\odot}\right)$ between the reconstructed event direction and the direction of the Sun.

\subsection{PMT Energy Response}

For the detector modeling, a knowledge of the PMT energy response is needed. The characterization of the NCD energy response is not required for the global solar neutrino oscillation analyses, due to the fact that physics interpretation is done in the active neutrino flux space, rather then using a predicted number of NC events from the NCD array.

The PMT energy response function for electrons is described by a Gaussian function:

$$
R_{e}\left(T_{e}, T\right)=\frac{1}{\sqrt{2 \pi} \sigma_{T}} \exp \left[-\frac{\left(T_{e}-T\right)^{2}-\Delta_{T}}{2 \sigma_{T}^{2}}\right]
$$

where $T_{e}$ is the true electron kinetic energy, $T$ is the observed electron kinetic energy, $\Delta_{T}$ is the energy offset. The latter is zero when the detector is correctly calibrated. Here the standard deviation, $\sigma_{T}$, depends on the true electron energy and is given by:

$$
\sigma_{T}=a+b \sqrt{T_{e}}+c T_{e}
$$

The parameters $a, b$ and $c$ for the three phases of SNO can be found in $[48,52,53]$, and are given in Table 4.1. 
Table 4.1: Electron energy response parameters for the three phases of SNO.

\begin{tabular}{cccc}
\hline \hline Parameter & SNO-I & SNO-II & SNO-III \\
\hline $\mathrm{a}$ & -0.0684 & -0.131 & -0.2955 \\
$\mathrm{~b}$ & 0.331 & 0.383 & 0.5031 \\
$\mathrm{c}$ & 0.0425 & 0.03731 & 0.0228 \\
\hline \hline
\end{tabular}

The PMT response function for neutrons during the pure $\mathrm{D}_{2} \mathrm{O}$ (SNO-I) phase represents a response of the detector to a monoenergetic $\gamma$ ray, and is described by a single Gaussian function:

$$
R_{n}(T)=\frac{1}{\sqrt{2 \pi} \sigma_{\gamma}} \exp \left[-\frac{\left(T_{\gamma}-T\right)^{2}}{2 \sigma_{\gamma}^{2}}\right]
$$

with a fixed mean kinetic energy $T_{\gamma}=5.646 \mathrm{MeV}$ and a standard deviation $\sigma_{\gamma}=1.11 \mathrm{MeV}$.

During the salt phase (SNO-II), the PMT energy response for neutrons is described by a more complicated function to represent a response of the detector to multiple $\gamma$ rays produced after neutron capture on chlorine and, to less extent, to a monoenergetic $\gamma$ ray from neutron capture on deuteron:

$$
R_{n}(T)=\frac{q}{\sqrt{2 \pi} \sigma_{\gamma_{1}}} \exp \left[-\frac{\left(T_{\gamma_{1}}-T\right)^{2}}{2 \sigma_{\gamma_{1}}^{2}}\right]+\frac{1-q}{\sqrt{2 \pi} \sigma_{\gamma_{2}}} \exp \left[-\frac{\left(T_{\gamma_{2}}-T\right)^{2}}{2 \sigma_{\gamma_{2}}^{2}}\right]
$$

with a weight $q=0.5647$, mean kinetic energies $T_{\gamma_{1}}=5.9094 \mathrm{MeV}$ and $T_{\gamma_{2}}=$ $6.8338 \mathrm{MeV}$ and standard deviations $\sigma_{\gamma_{1}}=1.2037 \mathrm{MeV}$ and $\sigma_{\gamma_{2}}=1.4100 \mathrm{MeV}$.

The PMT response functions described in this sections are used in Chapters 7 and 8 to predict the expected number of neutrino yields to be observed inside the SNO detector under the assumptions of two-neutrino mixing and weakly mixed 
sterile neutrino hypotheses, respectively. 


\section{Chapter 5}

\section{Data Selection and Livetime}

The data at SNO are collected as a series of runs that must meet certain selection criteria in order to be considered for physics analyses. The raw data during neutrino running consists mostly of instrumental and radioactive background events. The average trigger rates during the salt and the NCD phases of the experiment were about $20 \mathrm{~Hz}$ and $30 \mathrm{~Hz}$, respectively; whereas the number of expected neutrino interaction in the detector is only 10 per day. Thus, special care has to be taken in order to ensure the quality of the data. Run testing is performed both by SNO main analysis software called SNOMAN, and via human inspection. All runs that passed the run selection criteria are further processed, where instrumental background events and bursts are removed by applying a series of preselected cuts. While instrumental background cuts reject only noise events from the data set, the burst cuts remove actual periods of time when the detector was unstable and they directly affect the detector livetime. The precise knowledge of the detector livetime is crucial for the solar neutrino analysis, since the recorded number of neutrino events at SNO are interpreted in terms of neutrino 
fluxes. The following section describes the selection criteria for the data during the NCD (SNO-III) phase. The final section summarizes cuts applied to the data that affect the detector livetime and the calculation of the total livetime for the NCD data set.

\subsection{Run Selection}

In order to ensure the best quality of data taken, a series of run selection criteria are applied at SNO. The run selection process is carried by doing a human inspection of the shift reports, that are written by detector operators and contain important information on the detector condition, hardware, software, data acquisition system and laboratory environment during data taking. Each shift report is read by two members of the run selection committee and checked against the applicable selection criteria. Furthermore, important parameters for each neutrino run are extracted by two SNOMAN processors as described in the following section.

\subsubsection{FPS and SPS checks of runs}

Two tools are embedded in SNOMAN to extract important information about runs:

1. The First Pass Run Selection (FPS) processor

The FPS processor runs on the raw data files and extracts the following parameters:

- Run type 
This information is obtained from the run header, with the list of specific bits associated with each run. In addition to the bits that are required for normal neutrino running, sometimes an Unusual Circumstances (UC) bit could be preset by the detector operator, in case that some irregular circumstances occurred during the data taking (for example, an increase of temperature in the detector, a tour of visitors in the area above the detector, etc...).

- Trigger mask

Each run is checked for appropriate triggers and their threshold values required for normal neutrino running.

- DQXX tests

FPS checks if the minimum allowed number of crates and PMTs were online for each run.

- NQXX test

FPS checks if the minimum allowed number of NCDs was running with nominal settings.

- Run Time Test

The raw livetime is reported by FPS as a difference in time between the last and the first events in the run.

2. The Second Pass Run Selection (SPS) processor

The SPS processor assesses the data quality by performing a number of checks on raw data:

- The Run Header Check 
The processor compares the run header time with the first event time from the data stream. The run header time is obtained from the SNO Hardware and Real Control program that is used to monitor the data stream, hardware and to operate the detector. The event times from the data stream are measured with the oscillator $10 \mathrm{MHz}$ clock.

- Event Separation Check

The minimum time interval allowed between two events at SNO is constrained by the width of the trigger window. Whenever the event separation time is smaller than the minimum allowed value, both the timing of the events and the overall livetime could be inaccurate.

- The $10 \mathrm{MHz}$ Clock Check

Each event in the data has its associated identification number called General Time Identification (GTID). This test verifies whether the 10 $\mathrm{MHz}$ clock time increases with the increasing GTID of the event or not.

- The Rate Checks

A run fails if the averaged PMT and NCD rates of run are greater than the thresholds for unreasonably high rates, or if more than $20 \%$ of the run is above those thresholds.

- The Pulsed Global Trigger (PGT) Rate Check A run fails if the PGT average rate deviates by more than $2 \%$ from its nominal rate of $5 \mathrm{~Hz}$.

- The Sync Clear Check

Runs that have more than $1 \%$ of channels out of sync are rejected from the run list. 
- The Occupancy Check

This test records the number of zero occupancy tubes of each type (Normal, OWL, BUTT and NECK) and compares them to the maximum allowed values.

- The Trigger Rate Check

This test checks the mean crate occupancy and event rates for the ESUM $^{1}$ high trigger. If it finds a significantly low mean crate occupancy or average event rate below $1 \mathrm{~Hz}$ for more than $30 \%$ of the events, it fails a run.

- The Analog Measurement Board (AMB) Check

This test removes runs with a high rate for AMB integral pedestals.

- The QLX bit Check

Runs that do not have correctly set $\mathrm{QLX}^{2}$ bits are rejected.

A run that fails any of the FPS or SPS checks is discarded from the standard solar neutrino analysis.

\subsubsection{Selection of the Final Data Set}

The following criteria must also be met in order to use a run in the solar neutrino analysis:

1. Run Type

Run must be flagged as a neutrino run. Source and laser calibration runs, maintenance runs during detector repairs or electronic calibrations, runs

\footnotetext{
${ }^{1}$ ESUM is a trigger based on the charge that is deposited in PMTs.
}

${ }^{2} \mathrm{QLX}$ is a low gain charge measurement from PMTs. 
under unusual circumstances that could affect quality of data (for example, power outgages, calibration sources inside the detector, opened gate valve, etc...) are all rejected.

\section{Run Duration}

Run length must be greater that $30 \mathrm{~min}$, and its total deadtime fraction must be smaller than $20 \%$.

3. $\mathrm{D}_{2} \mathrm{O}$ circulation

Runs where $\mathrm{D}_{2} \mathrm{O}$ is circulating are permitted only if there is no production of light due to the circulation. Usually, a lot of light inside the detector is observed during runs when the circulation starts or ends, therefore these runs are rejected from the analysis.

4. Bubblers

Sometimes the heavy water is filled with air in order to determine the level of the water. The bubbles of air cause a great amount of light inside the detector, compromising the data quality. Runs with the bubblers flag are discarded from the run list.

5. Assays

A type of sampling of heavy water called Sample Line (SL) Assay produces light inside the detector. Runs flagged with the SL bit are rejected from the analysis.

6. Deck Temperature

The temperature in the area of the deck must be lower that $20.5^{\circ}$ for a good neutrino run. 
7. Deck Activity

No disruptive activity on deck is allowed for good neutrino runs.

\section{Compensation Coils}

The compensation coils installed at SNO to compensate the vertical component of the Earth magnetic field must be on and with the correct settings for each good neutrino run.

9. Online Electronics

All crates must be online, with the right voltages, and with the triggers enabled. The NCD system must be on and running under correct settings.

10. Event Rate

The average PMT event rate must not be greater than $60 \mathrm{~Hz}$ for more than $20 \%$ of the run duration. The average rate of NCD shaper ${ }^{3}$ events must not be greater than $1 \mathrm{kHz}$. The ratio of the NCD MUX ${ }^{4}$ and shaper events must be less than $90 \%$.

\subsection{Livetime}

SNO experiment is a real-time detector which measures solar neutrino interaction rates. The measurements of the solar neutrino interaction rates are translated into solar neutrino fluxes, with the knowledge of the precise time exposure of the detector: its livetime. In addition to the precise determination of the total time exposure of the detector, the accurate determination both of the separate day

\footnotetext{
${ }^{3}$ Shaper/ADC card is a part of the NCD data acquisition system that measures the total energy deposited by the signal.

${ }^{4} \mathrm{MUX}$ stands for four multiplexers in the data acquisition system that control the signal from the NCD to the digital oscilloscopes.
} 
and night livetimes, and the differential livetime as a function of the solar zenith angle are critical for the day-night asymmetry measurements at SNO, and for the physics interpretation of the SNO results, and in particular, for the extraction of the neutrino oscillation parameters.

\subsubsection{The Clocks}

There are two independent clock systems that are used at SNO to precisely determine the time of every event observed in the detector. The main clock at SNO is the $10 \mathrm{MHz}$ clock. This is the oscillator clock which is tied to the Global Position System (GPS), and thus synchronized with the Universal Coordinated Time (UTC). Another not less important feature of this clock is its long time accuracy due to the synchronization with the GPS. The clock time is stored on a 53-bit counter that rolls over after a period of 28 years, which is much longer that the total livetime of the experiment. Its accuracy is about $100 \mathrm{~ns}$. The 10 $\mathrm{MHz}$ clock signal is transmitted to the surface of the detector via approximately four kilometers of fibre-optic cable. The propagation time of the clock signal to the underground detector is monitored on an hourly basis in order to determine the time delay occurring during the process of transmission. The times measured by the $10 \mathrm{MHz}$ clock are the primary event times used in the data analysis.

The second independent clock used at SNO is the $50 \mathrm{MHz}$ clock. The $50 \mathrm{MHz}$ oscillator is situated underground and it is not synchronized with the GPS. The times determined by this clock are stored on 43-bit counter that has a roll-over period of only two days. However, this clock is more precise than the $10 \mathrm{MHz}$ clock and it is used for the measurements of the times between the detected events. Its second purpose is a verification of the livetimes measured by the $10 \mathrm{MHz}$ clock. 
The time from this clock is also used to rebuild the primary livetimes of runs when the $10 \mathrm{MHz}$ clock was not functioning properly. It has been shown that this clock runs actually at $49.9995 \mathrm{MHz}$ [54] and introduces a very small difference between the times measured by these two clocks.

\subsubsection{Raw Livetimes}

The primary livetime of SNO is measured with the $10 \mathrm{MHz}$ clock. The raw livetime of the run is a period of time elapsed between the last and the first events recorded. The events at SNO are sometimes written out of order, therefore special care has to be taken in determining this time interval. There is a small bias introduced in the livetime calculation due to the fact that there could be a small period at the beginning and towards the end of a run, where no events are recorded. However, this bias is never greater that $0.4 \mathrm{~s}$ per run, since random pulser at SNO runs at the frequency of $5 \mathrm{~Hz}$. For the complete data set of the NCD phase containing a total of 1995 runs, the possible bias introduced is not greater than $798 \mathrm{~s}$ or $0.0025 \%$ of the raw livetime. The raw livetime distribution for the NCD phase of the experiment is tabulated in Table 5.1.

The independent verification of the raw livetime is performed by the $50 \mathrm{MHz}$ clock, which is not as accurate as the $10 \mathrm{MHz}$ clock, since its time is not synchronized with the GPS system. In cases where the $10 \mathrm{MHz}$ clock was broken (a total of 24 runs in the NCD phase), the $50 \mathrm{MHz}$ clock was used to rebuild the primary run times and not for verification. For the complete NCD data set, the differences in the raw livetimes measured by these two clocks are presented in Figure 5.1. The plot shows a very good agreement in the livetimes measured by the two independent clocks. For the majority of the runs the difference is not 


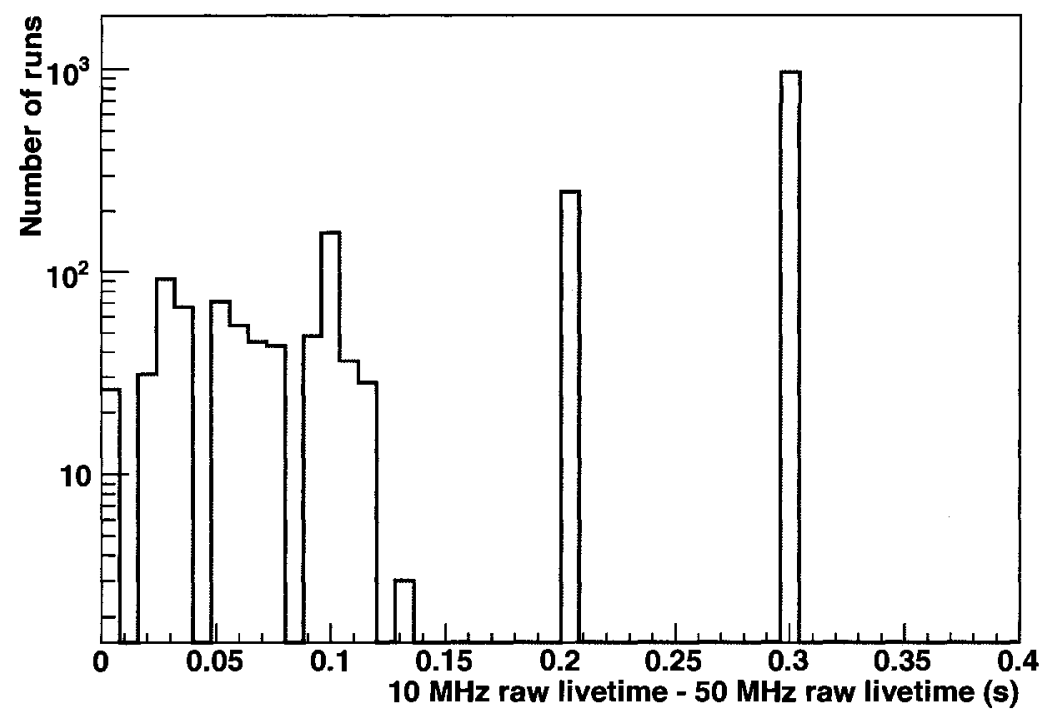

Figure 5.1: Distribution of the difference between the raw livetimes measured by the $10 \mathrm{MHz}$ and $50 \mathrm{MHz}$ clocks for 1995 runs of the NCD data set.

greater than $0.3 \mathrm{~s}$. Since $50 \mathrm{MHz}$ clock runs at slightly lower frequency, the expected difference between the times measured by the $10 \mathrm{MHz}$ and $50 \mathrm{MHz}$ clocks should be positive, which can be seen in Figure 5.1. There was one run where the observed discrepancy was over $200 \mathrm{~s}$. The source of this large difference was not fully understood and the run was removed from the neutrino runlist. A possible explanation for the large difference between the times measured by the two clocks could be due to the error in reading the timing information for the events [55].

\section{PGT Verification}

In addition to the $50 \mathrm{MHz}$ clock, the verification of the calculated livetime is performed by doing the Pulsed Global Trigger (PGT) analysis. The PGT is a random pulser that runs at a frequency of $5 \mathrm{~Hz}$, reads the state of the detector 
and it is driven by the $50 \mathrm{MHz}$ clock. The livetime estimate is accomplished by simply counting the number of PGT events in the data set and dividing it by 5 . Although the PGT livetime estimate is not completely independent from the time determined by the $50 \mathrm{MHz}$ clock, it is still a very useful tool for the verification of the livetimes, because the period of time obtained from the PGT events does not depend on whether events are written onto tape with the correct times or not. The smallest time interval measured by this method is $0.2 \mathrm{~s}$.

There are few complications associated with the livetime estimate using the PGT. In case a PGT event coincides with the trigger for a real event in the detector, it will be lost. During the first phase of SNO, this occurred approximately once in 40000 events during normal running [56]. If the rate is increased (e.g. during the period of bursts), the number of lost events is larger. During the NCD phase of the experiment, one out of every 20000 PGT events was lost due to coincidence with other events [57]. The further complication originates from the process called the NHIT Monitor, which surveys the NHIT triggers by causing channels on a crate to trigger and thus steals the PGT events. The events produced by this process are called pedestals. During the NCD phase of SNO, the NHIT Monitor was running much more frequently than in previous phases. It was run either on crate 18 or for the majority of the time on crate 10, every 30 minutes. The PGT events lost due to the NHIT Monitor running are recovered in the analysis by counting the pedestal events on the crate where the NHIT Monitor was active. The PGT livetime verification for the NCD phase is shown in Figure 5.2. Some of the discrepancies seen in the Figure 5.2 are due to the periods of burst where PGT events were lost or to faulty livetime calculation from the $10 \mathrm{MHz}$ clock.

The raw livetimes obtained by the two clocks and PGT events are presented 
Table 5.1: Summary of raw livetimes for the NCD data set.

\begin{tabular}{lc}
\hline \hline Measurement & Duration (days) \\
\hline Raw 10 MHz Livetime & 392.88 \\
Raw 50 MHz Livetime & 392.87 \\
Raw PGT Livetime & 392.86 \\
\hline \hline
\end{tabular}

in Table 5.1.

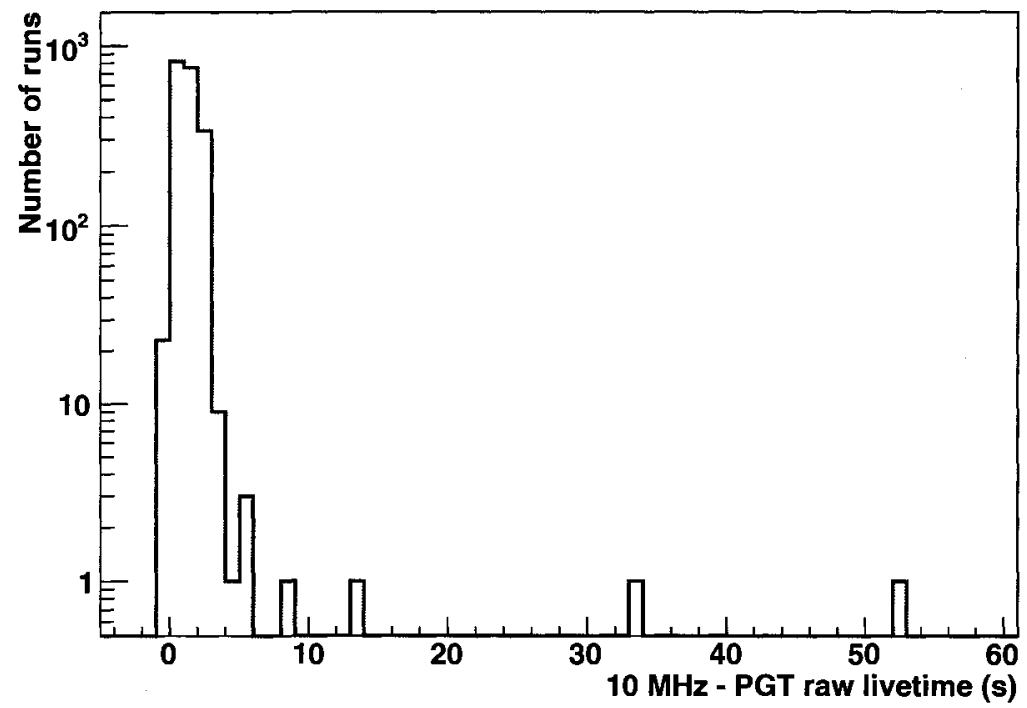

Figure 5.2: Distribution of the $10 \mathrm{MHz}$ minus PGT estimated raw livetimes for 1995 runs of the NCD data set.

\subsubsection{The Burst Cuts}

In addition to the low level instrumental cuts that remove non-physical events from the data set and do not affect the livetime, SNO implements a series of burst cuts. The burst cuts remove periods of time from the data set when burst of events in coincidence, and within some small time window, are observed; thus 
they directly affect the livetime calculation. The following burst cuts are applied during the NCD phase:

\section{- Retrigger Cut}

The retrigger cut removes $5 \mu$ s of livetime after each event followed by at least one event within the same time window. This cut prevents retriggering events from the detector due to the pickup that follows any event having high charge or high number of PMT hits.

\section{- NHIT Burst Cut}

The NHIT burst cut removes bursts of 6 or more events occurring within a time window of $4 \mathrm{~s}$ that produce at least $40 \mathrm{PMT}$ hits. This cut removes most of the instrumental background events excluding retriggers, but it also cuts some physical background events (for example neutrons following muons inside the detector).

\section{- Muon Follower Short Cut}

At the depth of the SNO detector, the number of expected muons inside the PMT array is about 70 per day [49]. Spallation products from muon interactions in heavy water create background to neutrino signal. Therefore, all events in time window of $20 \mathrm{~s}$ following a muon are tagged by this cut. The $20 \mathrm{~s}$ time window is sufficient to account for almost three lifetimes of ${ }^{16} \mathrm{~N}$, which is created by muon capture on ${ }^{16} \mathrm{O}[56]$ in $\mathrm{D}_{2} \mathrm{O}$.

\section{- Missed Muon Follower Short Cut}

Neutron events originated from interactions of muons that are unidentified by the muon tag, atmospheric neutrinos and other processes are tagged by this cut. All events at SNO occurring in $250 \mathrm{~ms}$ time window after an event 
with more than 150 PMT hits are removed from the data that are used in the neutrino analysis.

- The Shaper Burst Cut

NCD shaper events that occur in burst of 4 or more events and within 0.1 $\mathrm{s}$ are removed by this cut.

- The Mux Burst Cut

NCD mux events that occur in burst of 4 or more events and within $0.1 \mathrm{~s}$ are removed by this cut.

- Shaper Overflow Cut

NCD shaper events with ADC value 2047 are shaper overflow events. The shaper overflow cut tags all events in the time window starting $15 \mu$ s before, and $5 \mathrm{~ms}$ after the shaper overflow event [57].

- Run Boundary Cut

If it is not clear that both PMT and NCD systems are running, then the events at the start and end of a run are tagged by this cut. In this case, the start of a run is not defined by the first event recorded, but by a point in time $1.1 \mathrm{~s}$ after the first PMT or NCD event. Similarly, the end of the run is defined by a point in time $1.1 \mathrm{~s}$ before the last PMT or NCD event [58].

- NCD General Record (NGR) Cut

The NCD General Record events are NCD events that have triggers from both the shaper and mux. These events are not physical events, but pulses caused by the NCD system when NCD data records are inserted into the data stream. The NGR cut removes periods of time $30 \mathrm{~ms}$ before and $5 \mathrm{~ms}$ 
after each NGR event.

\subsubsection{The Dead Times}

The periods of individual cut times obtained by the burst cuts described in the previous section are measured by the $10 \mathrm{MHz}$ clock and stored in the Run Logger Dead Time (RLDT) data bank. The Livetime Summary Processor (LSP) of SNOMAN accesses the individual cut times stored in the RLDT bank, and calculates the total combined deadtime. The combined deadtime is, in general, smaller than the sum of the individual time cuts because various cuts can overlap between each other. The distribution of the combined deadtime for 1995 neutrino runs during the NCD phase is shown in Figure 5.3. The summary of the raw livetimes, in-

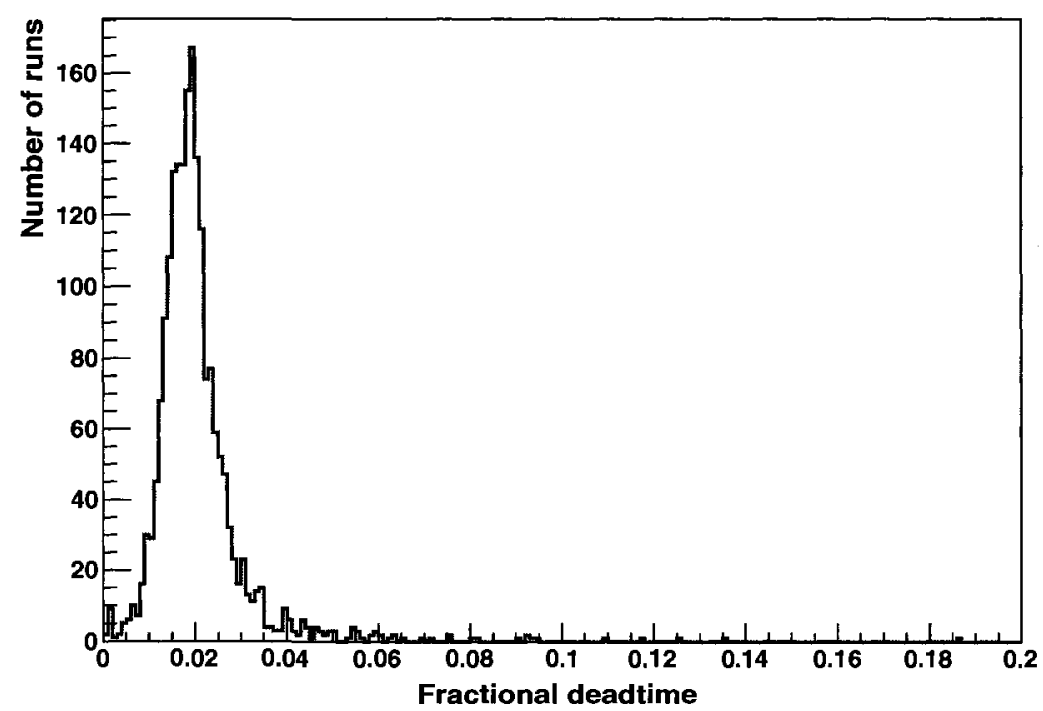

Figure 5.3: Distribution of the combined deadtime for the data set during the NCD phase consisting of 1995 runs.

dividual cut times, total non-overlapped deadtime and final deadtime corrected 
livetime, that are obtained from the LSP processor, is presented in Table 5.2. The combined deadtime is less than $2 \%$ of the raw livetime for the NCD data set. The largest contribution to this deadtime is due to the short muon follower cut, which cuts about $1.6 \%$ of the raw livetime, while the contributions from the retrigger and shaper overflow cuts are almost negligible.

Table 5.2: Summary of raw livetime, individual cuts, combined deadtime and corrected livetime for the NCD phase data set.

\begin{tabular}{lcc}
\hline \hline Measurement & Duration & Fraction of Raw Livetime \\
\hline Raw 10 MHz Livetime & 392.88 days & - \\
Raw 50 MHz Livetime & 392.87 days & - \\
\hline Retrigger Cut & $55.92 \mathrm{~s}$ & $1.65 \times 10^{-4 \%}$ \\
NHIT Burst Cut & $20025.9 \mathrm{~s}$ & $0.059 \%$ \\
Shaper Burst Cut & $46707.75 \mathrm{~s}$ & $0.138 \%$ \\
Mux Burst Cut & $59081.55 \mathrm{~s}$ & $0.174 \%$ \\
Muon Follower Short Cut & 6.38 days & $1.62 \%$ \\
Missed Muon Follower & $21230.03 \mathrm{~s}$ & $0.063 \%$ \\
Run Boundary Cut & $23032.33 \mathrm{~s}$ & $0.068 \%$ \\
Shaper Overflow Cut & $24.61 \mathrm{~s}$ & $7.25 \times 10^{-5} \%$ \\
NCD General Record Cut & $8670.69 \mathrm{~s}$ & $0.026 \%$ \\
\hline Combined Deadtime & 7.71 days & $1.96 \%$ \\
Corrected Livetime & 385.17 days & $98.04 \%$ \\
\hline \hline
\end{tabular}

\subsubsection{Uncertainties}

The method of livetime estimation that counts the number of PGT events has been used to estimate the uncertainty in the livetime calculation, and also to verify the individual and combined dead times calculated by the LSP processor. Since the burst cuts tag all events in the data stream, each cut time is estimated by counting the number of PGT and pedestal events tagged by the appropriate 
cut, and divided by 5 . The combined deadtime verification is obtained by counting all the PGT and pedestal events that are tagged by at least one of the burst cuts.

Table 5.3: Comparison of cut times percentages of the raw livetime calculated by LSP and by PGT.

\begin{tabular}{lccc}
\hline \hline Measurement & RLDT Cut Time & PGT Cut Time & Difference \\
\hline Retrigger & $1.65 \times 10^{-4 \%}$ & $0.001 \%$ & $0.0008 \%$ \\
NHIT Burst & $0.059 \%$ & $0.059 \%$ & $0 \%$ \\
Shaper Burst & $0.138 \%$ & $0.135 \%$ & $0.003 \%$ \\
Mux Burst & $0.174 \%$ & $0.174 \%$ & $0 \%$ \\
Muon Follower Short & $1.62 \%$ & $1.62 \%$ & $0 \%$ \\
Missed Muon Follower & $0.063 \%$ & $0.063 \%$ & $0 \%$ \\
Run Boundary & $0.068 \%$ & $0.098 \%$ & $0.03 \%$ \\
Shaper Overflow & $7.25 \times 10^{-5} \%$ & $6.36 \times 10^{-5} \%$ & $-0.89 \times 10^{-5} \%$ \\
NCD General Record & $0.026 \%$ & $0.026 \%$ & $0 \%$ \\
\hline Combined Cut Time & $1.96 \%$ & $1.99 \%$ & $0.03 \%$ \\
\hline
\end{tabular}

The comparison between the cut times obtained from the LSP and PGT verifications is summarized in Table 5.3. An excellent agreement is achieved between the two methods of deadtime estimation, except for the run boundary cut where a difference of $0.03 \%$ is observed. The source of this discrepancy is very small, but not understood, therefore it is treated as a systematic uncertainty on the livetime calculation. The distribution of the differences between the LSP and PGT cut corrected livetimes is shown in Figure 5.4. The corrected livetimes from the LSP and PGT for the total NCD data set are summarized in Table 5.4. The final livetime systematic uncertainty, which is determined by the difference between the LSP and PGT corrected livetimes, is $0.036 \%$. 


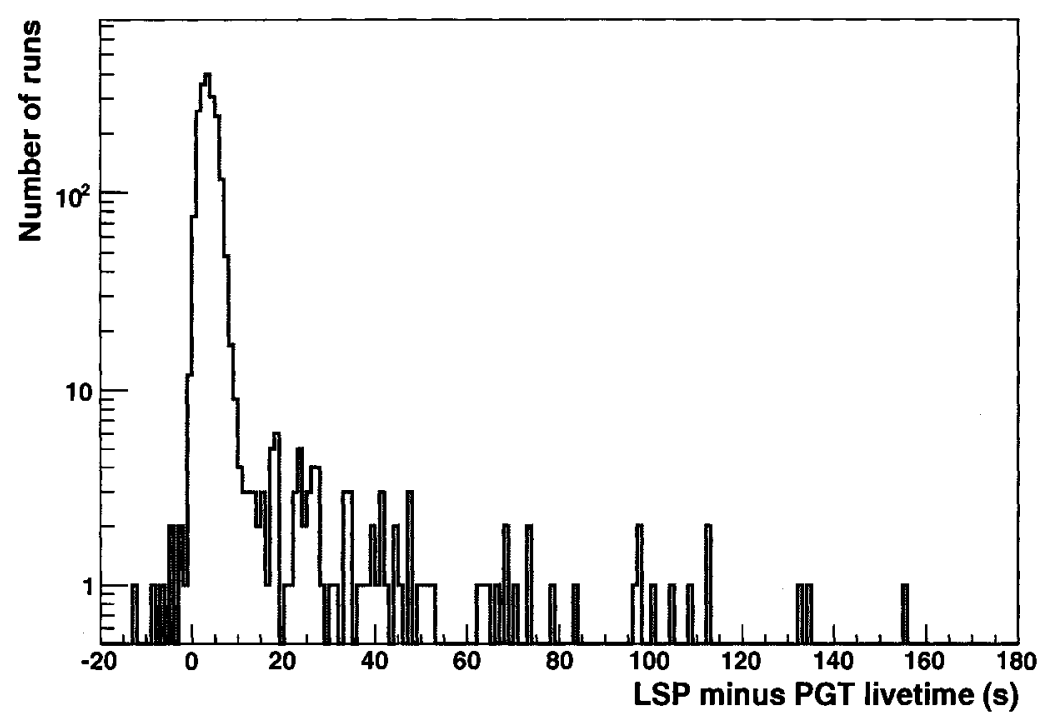

Figure 5.4: Distribution of the difference between the cut corrected livetimes from LSP and PGT, for 1995 runs of the NCD data set.

Table 5.4: Summary of the corrected livetimes from LSP and PGT for the NCD data set.

\begin{tabular}{ccc}
\hline \hline LSP Corrected Livetime & PGT Corrected Livetime & Difference. \\
\hline 385.17 days & 385.03 days & $0.036 \%$ \\
\hline \hline
\end{tabular}




\subsubsection{Livetime as a Function of Zenith Angle}

Day-night analysis at SNO requires knowledge of the separated livetimes for day and night. The day and night livetime bins are defined by the values of the solar zenith angle $\theta_{\text {sun }}$, which is the angle between the detector zenith and the position of the Sun. The LSP stores the livetime in 24 bins in terms of the $\cos \theta_{\text {sun }}$, where day is defined by a period of time when $\cos \theta_{\text {sun }}>0$. Summary of the day and night livetimes calculated from LSP and verified by PGT counting is tabulated in Table 5.5. It can been seen in Table 5.5 that the detector was more live during the night (about $54.6 \%$ of the total livetime). This is due to the fact that both the calibrations and repairs of the detector are performed during the day at SNO.

Table 5.5: Summary of day and night corrected livetimes from LSP and PGT for the NCD data set.

\begin{tabular}{lccc}
\hline \hline Zenith Bin & LSP Livetime & PGT Livetime & Difference \\
\hline Day & 176.59 days & 176.52 days & $0.040 \%$ \\
Night & 208.58 days & 208.51 days & $0.036 \%$ \\
\hline Total & 385.17 days & 385.03 days & $0.036 \%$ \\
\hline \hline
\end{tabular}

In addition to these differential livetimes for day and night, a finer livetime binning is required for the extraction of the solar neutrino oscillation parameters. The solar neutrino survival probabilities for SNO have to be properly weighted by the real detector livetime distribution. This is achieved by producing the livetime in 480 solar zenith angle bins, which is shown in Figure 5.5. 
Zenith bin livetime distribution, NCD total data set

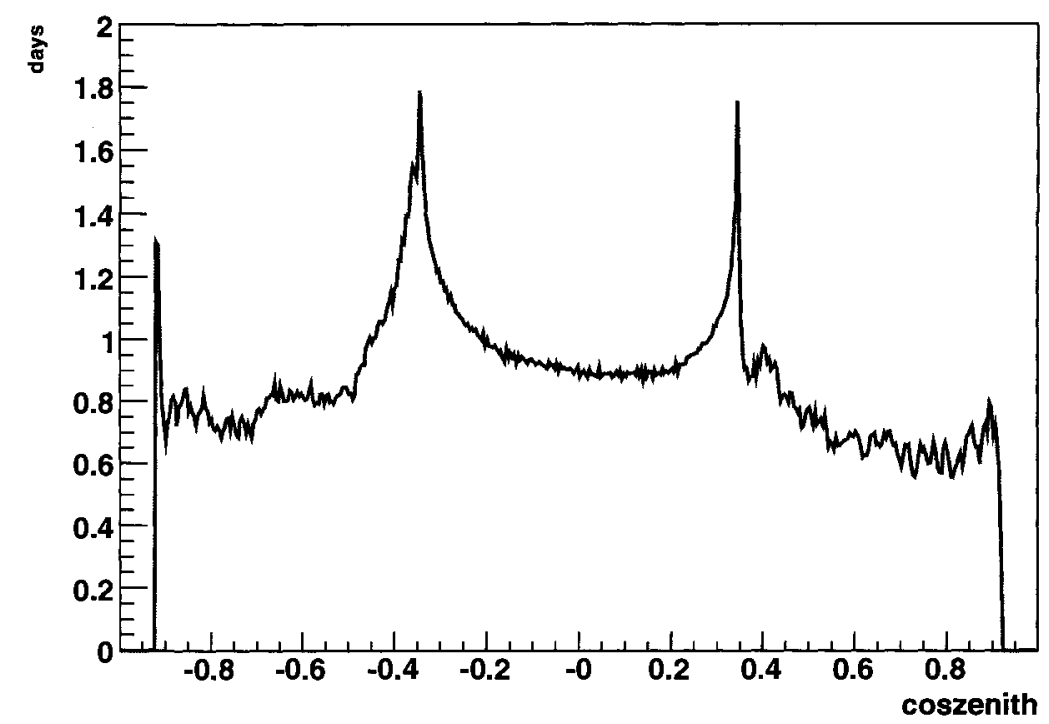

Figure 5.5: Corrected livetime of the NCD data set as a function of solar zenith angle.

\section{$5.3 \quad$ Results}

The final livetimes with their uncertainties for the NCD data set are summarized in Table 5.6. These numbers are going to be used by all analysts at SNO in

Table 5.6: Summary of final livetimes with their uncertainties for the NCD phase data set.

\begin{tabular}{ccc}
\hline Total & Day & Night \\
\hline$(385.17 \pm 0.14)$ days & $(176.59 \pm 0.07)$ days & $(208.58 \pm 0.07)$ days \\
\hline \hline
\end{tabular}

order to interpret forthcoming measurements from the NCD phase in terms of the solar neutrino fluxes, and also in this dissertation for the purpose of physics interpretation of these fluxes. Hence they are crucial for solar neutrino analyses that include the latest measurements from SNO. 
Now that a very technical aspect of this dissertation has been reviewed in detail, a physical interpretation of solar neutrino data including measurements from SNO, under the assumption of the two-neutrino and weakly mixed sterile neutrino hypotheses, are presented in the remaining chapters. 


\section{Chapter 6}

\section{SNO Data Samples}

In this chapter, detailed descriptions of the data samples from different phases of the SNO experiment, together with the associated systematic uncertainties that are relevant for our analyses, are presented.

\subsection{Data Samples}

SNO-I spectral data from 306 days of pure $\mathrm{D}_{2} \mathrm{O}$ running are used in a form of summed electron kinetic energy spectra for CC, ES, NC and background events, separately for day and night. The background consists of neutron background (NCBG) and low-energy background (LEB) events. There are 17 spectral bins of observed kinetic energy for each data set (day and night) starting at $5.0 \mathrm{MeV}$ up to $13.0 \mathrm{MeV}$ in steps of $0.5 \mathrm{MeV}$, and one integrated energy bin in the range of 13.0-20.0 MeV. These spectra are tabulated in Table 6.1. The neutron background spectrum, which has the same shape as the $\mathrm{NC}$ signal, originates from the photodisintegration neutrons produced outside and inside the acrylic vessel. The 
low energy background component of the spectrum has contributions from the three types of events: $\mathrm{D}_{2} \mathrm{O} \beta-\gamma, \mathrm{PMT} \beta-\gamma$ and $\mathrm{AV}-\mathrm{H}_{2} \mathrm{O} \beta-\gamma$ events. The NCBG and LEB background spectra are given in Tables 6.2 and 6.3, respectively. These two background spectra are needed in order to correctly calculate the expected number of signal events from the neutrino oscillation models. Statistical correlations between bins for the three signals from this phase are not needed for the analyses since the spectral data are used only as a sum CC+ES+NC. With the separation of the data into day and night samples, the matter induced neutrino transition (MSW effect), that is experienced by the neutrinos traversing matter inside the Earth, is included in the global solar oscillation analyses.

SNO-II data from 391 days of the salt phase running has been extracted as CC spectra, NC and ES integrated fluxes as shown in Tables 6.4 and 6.5 (separately for day and night). The CC spectra are divided into 16 spectral bins of observed kinetic energy from $5.5 \mathrm{MeV}$ up to $13.5 \mathrm{MeV}$ in steps of $0.5 \mathrm{MeV}$ and one integrated bin in the range of $13.5-20.0 \mathrm{MeV}$. The separation of $\mathrm{CC}, \mathrm{NC}$ and ES events performed by the SNO unconstrained signal extraction allows us to include important $\mathrm{CC}$ spectral shape information in the extraction of the neutrino mixing parameters. The unconstrained signal extraction separates the $\mathrm{CC}, \mathrm{NC}$ and ES signal events by utilizing event isotropy and angular distribution information, without any assumption about the shape of the ${ }^{8} \mathrm{~B}$ spectrum. The shape of the expected CC spectrum is directly affected by the survival probability of $\nu_{e}$. Therefore the inclusion of the spectral shape provides valuable information for constraining the neutrino oscillation model predictions. Statistical correlations $\rho_{i j}$ $(i, j=1,19)$ between the three signals are included in the analyses and two $19 \times 19$ statistical covariance matrices obtained from the signal extraction are tabulated 
Table 6.1: SNO-I day and night $\mathrm{CC}+\mathrm{NC}+\mathrm{ES}+$ backgrounds summed kinetic energy spectra given as number of events. The statistical errors for these data are calculated as square roots of the number of events in each spectral bin. The spectra are taken from Reference [59].

\begin{tabular}{ccc}
\hline \hline Spectral bin (MeV) & Day & Night \\
\hline $5.0-5.5$ & 191 & 301 \\
$5.5-6.0$ & 180 & 236 \\
$6.0-6.5$ & 163 & 205 \\
$6.5-7.0$ & 121 & 188 \\
$7.0-7.5$ & 104 & 177 \\
$7.5-8.0$ & 81 & 133 \\
$8.0-8.5$ & 70 & 92 \\
$8.5-9.0$ & 76 & 101 \\
$9.0-9.5$ & 49 & 72 \\
$9.5-10.0$ & 45 & 65 \\
$10.0-10.5$ & 36 & 47 \\
$10.5-11.0$ & 27 & 45 \\
$11.0-11.5$ & 17 & 31 \\
$11.5-12.0$ & 10 & 16 \\
$12.0-12.5$ & 5 & 14 \\
$12.5-13.0$ & 6 & 12 \\
$13.0-20.0$ & 5 & 7 \\
\hline \hline
\end{tabular}


Table 6.2: SNO-I day and night energy spectra for neutron background (NCBG). Columns labeled with $\sigma$ indicate the uncertainty on the associated number of background events. The spectra are obtained from Reference [59].

\begin{tabular}{ccccc}
\hline \hline $\begin{array}{c}\text { Energy bin } \\
(\mathrm{MeV})\end{array}$ & $\begin{array}{c}\text { NCBG } \\
\text { Day }\end{array}$ & $\begin{array}{c}\sigma_{\text {NCBG }} \\
\text { Day }\end{array}$ & $\begin{array}{c}\text { NCBG } \\
\text { Night }\end{array}$ & $\begin{array}{c}\sigma_{\text {NCBG }} \\
\text { Night }\end{array}$ \\
\hline $5.0-5.5$ & 10.3928 & 1.6092 & 15.9916 & 2.4809 \\
$5.5-6.0$ & 8.7606 & 1.3565 & 13.4801 & 2.0913 \\
$6.0-6.5$ & 6.0286 & 0.9335 & 9.2763 & 1.4391 \\
$6.5-7.0$ & 3.3867 & 0.5244 & 5.2112 & 0.8084 \\
$7.0-7.5$ & 1.5532 & 0.2405 & 2.3899 & 0.3708 \\
$7.5-8.0$ & 0.5815 & 0.0900 & 0.8947 & 0.1388 \\
$8.0-8.5$ & 0.1777 & 0.0275 & 0.2735 & 0.0424 \\
$8.5-9.0$ & 0.0443 & 0.0069 & 0.0682 & 0.0106 \\
$9.0-9.5$ & 0.0090 & 0.0014 & 0.0139 & 0.0022 \\
$9.5-10.0$ & 0.0015 & 0.0002 & 0.0023 & 0.0004 \\
$10.0-10.5$ & 0.0002 & 0.0000 & 0.0003 & 0.0000 \\
$10.5-11.0$ & 0.0000 & 0.0000 & 0.0000 & 0.0000 \\
$11.0-11.5$ & 0.0000 & 0.0000 & 0.0000 & 0.0000 \\
$11.5-12.0$ & 0.0000 & 0.0000 & 0.0000 & 0.0000 \\
$12.0-12.5$ & 0.0000 & 0.0000 & 0.0000 & 0.0000 \\
$12.5-13.0$ & 0.0000 & 0.0000 & 0.0000 & 0.0000 \\
$13.0-20.0$ & 0.0000 & 0.0000 & 0.0000 & 0.0000 \\
\hline \hline
\end{tabular}


Table 6.3: SNO-I day and night energy spectra for low energy Čerenkov background (LEB). Columns labeled with $\sigma$ indicate the uncertainty on the associated number of background events. The spectra are obtained from Reference [59].

\begin{tabular}{ccccc}
\hline \hline $\begin{array}{c}\text { Energy bin } \\
(\mathrm{MeV})\end{array}$ & $\begin{array}{c}\text { LEB } \\
\text { Day }\end{array}$ & $\begin{array}{c}\sigma_{\text {LEB }} \\
\text { Day }\end{array}$ & $\begin{array}{c}\text { LEB } \\
\text { Night }\end{array}$ & $\begin{array}{c}\sigma_{\text {LEB }} \\
\text { Night }\end{array}$ \\
\hline $5.0-5.5$ & 16.7125 & 5.5747 & 26.2490 & 8.5824 \\
$5.5-6.0$ & 0.9377 & 0.3584 & 1.4727 & 0.5413 \\
$6.0-6.5$ & 0.0479 & 0.0207 & 0.0752 & 0.0300 \\
$6.5-7.0$ & 0.0019 & 0.0009 & 0.0030 & 0.0013 \\
$7.0-7.5$ & 0.0001 & 0.0000 & 0.0001 & 0.0001 \\
$7.5-8.0$ & 0.0000 & 0.0000 & 0.0000 & 0.0000 \\
$8.0-8.5$ & 0.0000 & 0.0000 & 0.0000 & 0.0000 \\
$8.5-9.0$ & 0.0000 & 0.0000 & 0.0000 & 0.0000 \\
$9.0-9.5$ & 0.0000 & 0.0000 & 0.0000 & 0.0000 \\
$9.5-10.0$ & 0.0000 & 0.0000 & 0.0000 & 0.0000 \\
$10.0-10.5$ & 0.0000 & 0.0000 & 0.0000 & 0.0000 \\
$10.5-11.0$ & 0.0000 & 0.0000 & 0.0000 & 0.0000 \\
$11.0-11.5$ & 0.0000 & 0.0000 & 0.0000 & 0.0000 \\
$11.5-12.0$ & 0.0000 & 0.0000 & 0.0000 & 0.0000 \\
$12.0-12.5$ & 0.0000 & 0.0000 & 0.0000 & 0.0000 \\
$12.5-13.0$ & 0.0000 & 0.0000 & 0.0000 & 0.0000 \\
$13.0-20.0$ & 0.0000 & 0.0000 & 0.0000 & 0.0000 \\
\hline \hline
\end{tabular}


Table 6.4: SNO-II CC electron kinetic energy spectra for day and night with their statistical errors, expressed in units of equivalent $\mathrm{SSM}^{8} \mathrm{~B}$ fluxes. The spectra can be found in Reference [48].

\begin{tabular}{ccc}
\hline $\begin{array}{c}\text { CC electron kinetic } \\
\text { energy bin }(\mathrm{MeV})\end{array}$ & $\begin{array}{c}\text { Day } \\
\left(10^{6} \mathrm{~cm}^{-2} \mathrm{~s}^{-1}\right)\end{array}$ & $\begin{array}{c}\text { Night } \\
\left(10^{6} \mathrm{~cm}^{-2} \mathrm{~s}^{-1}\right)\end{array}$ \\
\hline $5.5-6.0$ & $0.205 \pm 0.032$ & $0.145 \pm 0.027$ \\
$6.0-6.5$ & $0.182 \pm 0.030$ & $0.164 \pm 0.027$ \\
$6.5-7.0$ & $0.153 \pm 0.028$ & $0.190 \pm 0.026$ \\
$7.0-7.5$ & $0.226 \pm 0.028$ & $0.180 \pm 0.024$ \\
$7.5-8.0$ & $0.198 \pm 0.025$ & $0.178 \pm 0.022$ \\
$8.0-8.5$ & $0.184 \pm 0.023$ & $0.164 \pm 0.019$ \\
$8.5-9.0$ & $0.124 \pm 0.018$ & $0.114 \pm 0.015$ \\
$9.0-9.5$ & $0.099 \pm 0.015$ & $0.126 \pm 0.015$ \\
$9.5-10.0$ & $0.110 \pm 0.015$ & $0.124 \pm 0.014$ \\
$10.0-10.5$ & $0.058 \pm 0.011$ & $0.067 \pm 0.010$ \\
$10.5-11.0$ & $0.070 \pm 0.012$ & $0.073 \pm 0.011$ \\
$11.0-11.5$ & $0.048 \pm 0.010$ & $0.039 \pm 0.007$ \\
$11.5-12.0$ & $0.042 \pm 0.008$ & $0.029 \pm 0.007$ \\
$12.0-12.5$ & $0.0088 \pm 0.0038$ & $0.018 \pm 0.005$ \\
$12.5-13.0$ & $0.0082 \pm 0.0040$ & $0.015 \pm 0.005$ \\
$13.0-13.5$ & $0.0025 \pm 0.0028$ & $0.0042 \pm 0.0025$ \\
$13.5-20.0$ & $0.014 \pm 0.005$ & $0.012 \pm 0.004$ \\
\hline \hline
\end{tabular}

in Tables 6.8 and 6.9 (for day and night).

SNO-III data from 385 days of NCD running are obtained from the signal extraction as integrated CC, ES and NC fluxes (averaged over day and night). The CC and ES energy spectra from the PMT array and NC events from both the NCD and PMT arrays were not constrained to the ${ }^{8} \mathrm{~B}$ spectrum shape. The signal extraction used the Markov-Chain Monte Carlo (MCMC) method with Metropolis-Hastings algorithm [60,61]. The MCMC allows for the simultaneous extraction of all three signals and the fit for the systematic uncertainties that are allowed to float within the constraints set by their nominal input values estimated 
Table 6.5: SNO-II ES and NC fluxes from a shape-unconstrained signal extraction for day and night. Fluxes are expressed in units of $10^{6}$ neutrinos $\mathrm{cm}^{-2} \mathrm{sec}^{-1}$ and are obtained from Reference [48].

\begin{tabular}{lcc}
\hline \hline Signal & Day flux & Night flux \\
\hline ES & $2.17 \pm 0.34$ (stat) \pm 0.14 (syst) & $2.52 \pm 0.32$ (stat) \pm 0.16 (syst) \\
NC & $4.81 \pm 0.31$ (stat) \pm 0.39 (syst) & $5.02 \pm 0.29$ (stat) \pm 0.41 (syst) \\
\hline \hline
\end{tabular}

Table 6.6: SNO-III CC, ES and NC fluxes with their statistical and systematical uncertainties from a shape-unconstrained signal extraction. Fluxes are expressed in units of $10^{6}$ neutrinos $\mathrm{cm}^{-2} \mathrm{sec}^{-1}$ and are obtained from Reference [51].

\begin{tabular}{lc}
\hline \hline Signal & Average flux \\
\hline CC & $1.67_{-0.04}^{+0.05}$ (stat $)_{-0.08}^{+0.07}$ (syst) \\
ES & $1.77_{-0.21}^{+0.24}$ (stat $)_{-0.10}^{+0.09}$ (syst) \\
NC & $5.54_{-0.31}^{+0.33}$ (stat $)_{-0.34}^{+0.36}$ (syst) \\
\hline \hline
\end{tabular}

Table 6.7: SNO-III statistical correlation coefficients for the CC, ES and NC fluxes. These coefficients can be found in Reference [62].

\begin{tabular}{c|ccc}
\hline \hline Signal & $\mathrm{CC}$ & $\mathrm{ES}$ & $\mathrm{NC}$ \\
\hline $\mathrm{CC}$ & 1.000 & 0.2376 & -0.1923 \\
$\mathrm{ES}$ & 0.2376 & 1.000 & 0.0171 \\
$\mathrm{NC}$ & -0.1923 & 0.0171 & 1.000 \\
\hline \hline
\end{tabular}


Table 6.8: SNO-II statistical correlation coefficients for day CC spectrum, ES and NC fluxes. These coefficients can be found in Reference [48].

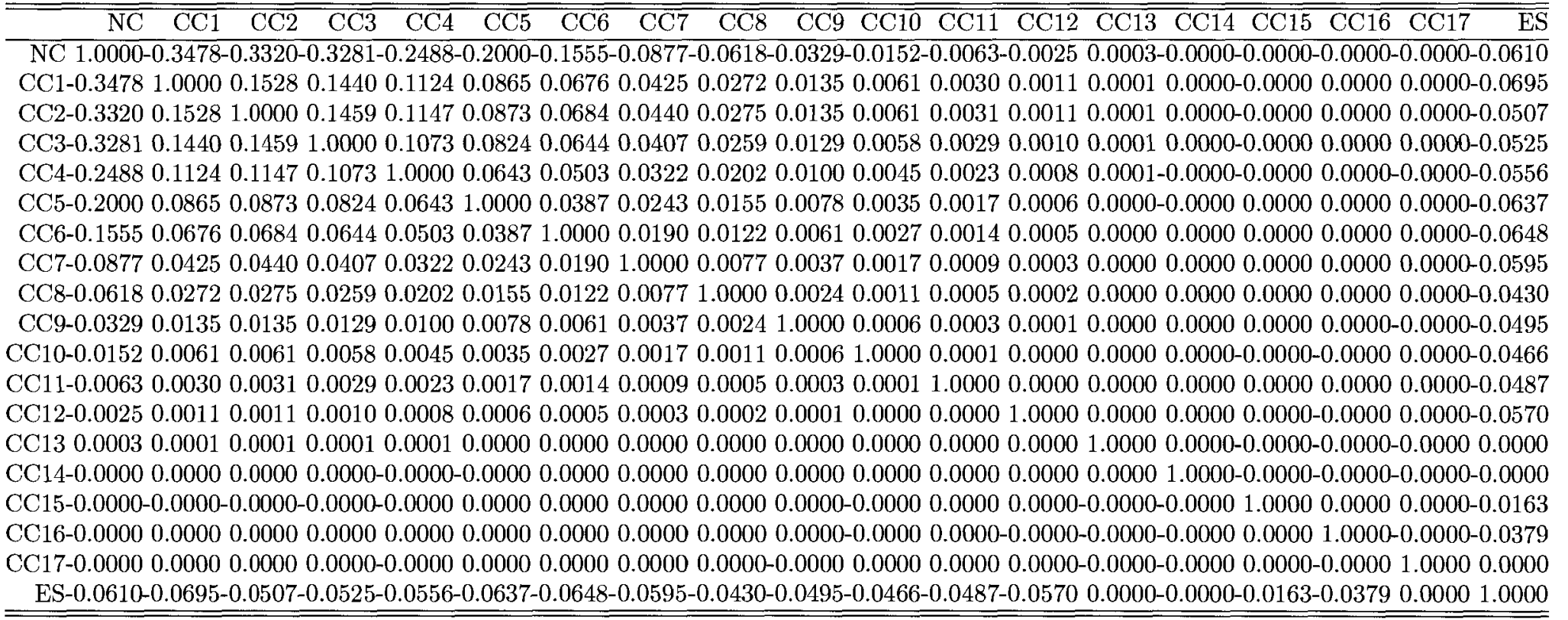


Table 6.9: SNO-II statistical correlation coefficients for night CC spectrum, ES and NC fluxes. These coefficients can be found in Reference [48].

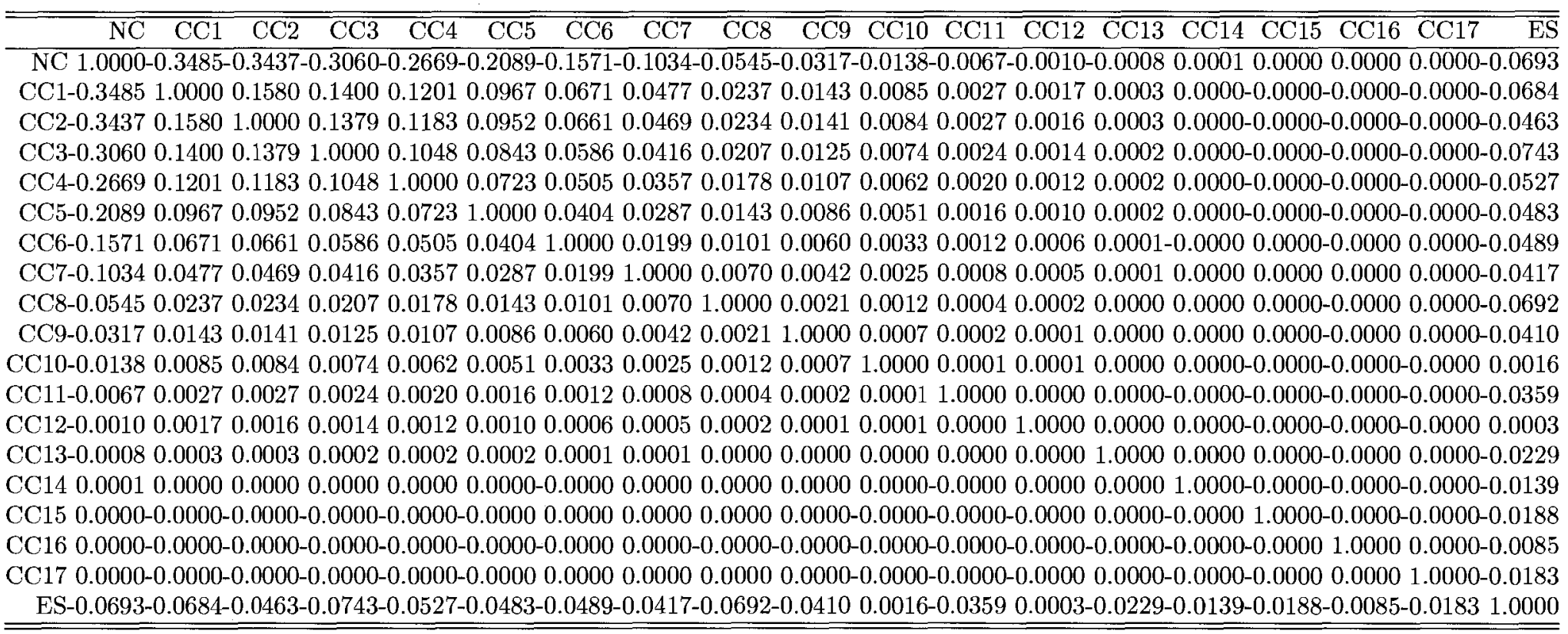


from calibration data. The CC, ES and NC integrated fluxes, as tabulated in Table 6.6, are determined under the assumption of the ${ }^{8} \mathrm{~B}$ neutrino spectrum shape from Winter et al. [63]. The statistical correlation coefficients $\rho_{i j}$ (where $i, j=1,3)$ between the three fluxes are tabulated in Table 6.7 .

\subsection{Systematic Uncertainties}

The dominant systematic uncertainties affecting the data for the three phases of SNO experiments are presented in this section. Some uncertainties affect the response function of the SNO detector (e.g. energy scale and energy resolution uncertainties), while others affect the prediction of the total number of events produced inside the detector (e.g. neutron capture efficiency and errors on neutrino interactions cross-sections). Description of the miscellaneous uncertainties that are not relevant to the analyses presented in this dissertation can be found elsewhere $[14,48,51,64]$. The systematic uncertainties described here are used to predict the expected number of signal events at the SNO location under the assumption of neutrino oscillation models.

\subsubsection{Neutron Capture Efficiency}

The probability for a neutron created inside the acrylic vessel $(r<600 \mathrm{~cm})$ to be captured on deuterium in pure $\mathrm{D}_{2} \mathrm{O}$ is $(29.9 \pm 1.1) \%$. Due to the radial $(r<550$ $\mathrm{cm})$ and energy threshold $(T>5 \mathrm{MeV})$ cuts, the actual neutron capture efficiency for the SNO-I data set is $(14.4 \pm 0.53) \%$. The neutron capture efficiency can also be calculated separately for events that meet only the fiducial volume cut. Then one can compute the portion of events that passes the energy event selection. The 
radial acceptance is defined as the number of neutrons, regardless of their energy, captured within $550 \mathrm{~cm}$ of the fiducial volume. This corresponds to a neutron capture efficiency of $(27.01 \pm 0.99) \%$. The neutron capture efficiency for $r<$ $550 \mathrm{~cm}$ is used to compute the predicted number of signal events at SNO under a given neutrino oscillation hypothesis. The requirement $T>5 \mathrm{MeV}$ further reduces the number of detected neutrons by $53.2 \%$. For SNO-II, where the salt was added to the heavy water to increase neutral current detection sensitivity, the neutron capture efficiency for the NC events above energy threshold of $T>5.5$ $\mathrm{MeV}$ and within the fiducial volume $(r<550 \mathrm{~cm})$ cut is $\left(40.7 \pm 0.5_{-0.8}^{+0.9}\right) \%$. The corresponding neutron capture efficiency for $r<550 \mathrm{~cm}$ is $(70.55 \pm 2.26) \%$ with a $57.69 \%$ of events above the energy threshold. For SNO-III, with the energy threshold of $T>6.0 \mathrm{MeV}$ and within the fiducial volume $(r<550 \mathrm{~cm})$, the neutron detection efficiency of the PMT is $(4.8+0.1) \%$. For the NCD array, the neutron capture efficiency is $(21.1 \pm 0.7) \%$. These two detection efficiencies were used by the signal extraction to extract the CC, ES and NC number of events, but they are not used in this thesis because we are testing the fluxes, not the number of events from the NCD phase. However, a relative uncertainty of $3.1 \%$ on the PMT + NCD weighted average detection efficiency is used in our analyses, because it affects the values of the expected fluxes for a given model hypothesis.

\subsubsection{Energy Scale}

One of the most important systematic uncertainty is the error on the energy scale in the response of the PMT. Due to the energy response of the detector described in Section 4.3, each event at SNO with a true total energy $E_{e}$ will be reconstructed with some observed energy $E$, which equals, on average, the true electron recoil 
energy. In case that the absolute energy scale of the detector has been miscalculated, the average observed energy $E$ for all the events will be equal to some energy shifted from its true value $E^{\prime}=E_{e}(1+\alpha)$. The parameter $\alpha$ represents the uncertainty in the total energy scale of the detector. This uncertainty affects the shape of the spectra at SNO, and more importantly, it alters the event acceptance. The latter effect results in either more or fewer events above the energy threshold; thus it directly effects the values of the neutrino fluxes measured by SNO. The values for the energy scale uncertainties obtained from calibration studies for the three phases of SNO are given in Tables 6.10, 6.11 and 6.12 .

\subsubsection{Energy Resolution}

While the PMT response functions are given by Equations (4.9), (4.11) and (4.12), the energy resolution is parameterized by the function:

$$
\frac{\Delta \sigma_{E}}{\sigma_{E}}=p+q\left(E_{e}-E_{16 \mathrm{~N}}\right)
$$

where $E_{16_{\mathrm{N}}}=6.13 \mathrm{MeV}$ is the energy of the photon produced by the ${ }^{16} \mathrm{~N}$ source. The ${ }^{16} \mathrm{~N}$ source is deployed in the detector to calibrate its response in energy. The ${ }^{16} \mathrm{~N}$ source is a good tool to produce electron through Compton scattering. The parameter $p$ is the relative energy resolution uncertainty at the energy of the ${ }^{16} \mathrm{~N}$ source, while $q=0.00401$ is the parameter used to interpolate the relative

energy resolution uncertainty between the energy of the ${ }^{16} \mathrm{~N}$ source and the 19.8 $\mathrm{MeV} \gamma$-ray produced by the pT source [64]. The pT source is used to calibrate the detector at high energy. The ${ }^{8} \mathrm{Li}$ source is another device used to calibrate the detector since ${ }^{8} \mathrm{Li}$ produces a continuous $\beta$ spectrum with an end point at 
about $14 \mathrm{MeV}$. The values of the energy resolution uncertainties for the three phases of SNO shown in Tables 6.10, 6.11 and 6.12 are evaluated at the energy $E_{e}=E_{16_{\mathrm{N}}}=6.13 \mathrm{MeV}$.

\subsubsection{Energy Non-Linearity}

The primary device for the energy calibration of the detector near the energy threshold is the ${ }^{16} \mathrm{~N}$ source. However, some small differential energy non-linearities can arise at higher energies where there is a lack of calibration data. The nonlinearity affects the overall shapes of the energy spectra extracted at SNO, and mostly arises from the varying detector electronic response via a change of channelto-channel cross-talk as a function of the number of incident Čerenkov photons. In order to estimate the shift in the energy scale as a function of the energy, the 19.8 $\mathrm{MeV} \gamma$-ray pT source is used. By linear interpolation between the results at ${ }^{16} \mathrm{~N}$ and $\mathrm{pT}$ energies for the SNO-I data set, the estimated non-linear shift in kinetic energy $\delta T$ as a function of the observed kinetic energy $T$ is given by $[59,65]$ :

$$
\delta T=\alpha \times 19.1 \times \frac{T-4.98}{13.61}(\text { in } \mathrm{MeV})
$$

where $\alpha$ is the $1 \sigma$ uncertainty in energy non-linearity (given in Table 6.10). It can be seen from Equation (6.2) that the error on the observed energy due to energy non-linearity equals to zero at $T=4.98 \mathrm{MeV}$ and then increases linearly with energy. For SNO-II data set, energy non-linearity uncertainty $\alpha$ is energy dependent (see Table 6.11) and parameterized by [66]:

$$
\alpha=\frac{(T-5.05)}{11.11}(\text { in } \%) \text {. }
$$




\subsubsection{Vertex Resolution and Accuracy}

The vertex resolution describes the distribution of the reconstructed event positions with respect to their true coordinate positions in the detector. It is modeled by a Gaussian function with small exponential tails [52]:

$$
R(x)=\frac{1-\alpha_{e}}{\sqrt{2 \pi} \sigma} \mathrm{e}^{-\frac{1}{2}(x-\mu)^{2} / \sigma^{2}}+\frac{\alpha_{e}}{2 \tau} \mathrm{e}^{-|x-\mu| / \tau}
$$

The uncertainty in the vertex resolution, which is defined as an uncertainty on the width of the Gaussian function given in Equation (6.4), has an overall small effect on the fitted number of events inside the fiducial volume.

The vertex accuracy represents the difference between the true and reconstructed event position. It can also be referred as a bias on the position. Radial scaling could then occur through position reconstruction or timing calibration uncertainties. The radial uncertainty is estimated to be around $1 \%$ of the radius for all three phases. The uncertainty on the vertex accuracy translates directly on an uncertainty on the fiducial volume (e.g. a $1 \%$ uncertainty in vertex accuracy at $r \sim 550 \mathrm{~cm}$ causes a $3 \%$ uncertainty on the fiducial volume). Thus the vertex accuracy strongly affects the total number of events reconstructed inside the fiducial volume. It is the second largest systematic uncertainty after the error on the energy scale.

\subsubsection{Sacrifice}

There is a fraction of good data at SNO that is lost due to instrumental and high-level cuts, as well as to failures in event reconstruction. The signal loss in the event selection and reconstruction is referred to as the sacrifice uncertainty. 
This effect is small, but it is nevertheless considered (both for day and night).

\subsubsection{Event Isotropy}

The uniformity of the distribution of the triggered PMT due to the solar neutrino events is described by the isotropy parameter $\beta_{14}=\beta_{1}+4 \beta_{4}$, where:

$$
\beta_{l}=\frac{2}{N(N-1)} \sum_{i=1}^{N-1} \sum_{j=i+1}^{N} P_{l}\left(\cos \theta_{i j}\right) .
$$

In this equation $N$ is the total number of triggered PMT associated with an event, $P_{l}$ is the Legendre polynomial of order $l$ and $\theta_{i j}$ is the angle between PMT $i$ and PMT $j$. The isotropy parameter $\beta_{14}$ is a powerful tool for separating electron (CC and ES) from neutron (NC) events during the salt phase of the experiment. It is evaluated by comparing calibration data from the ${ }^{16} \mathrm{~N}$ and ${ }^{252} \mathrm{Cf}$ neutron sources with Monte Carlo (MC) predictions and it is parameterized by a Gaussian function. Uncertainties on $\beta_{14}$ mean and resolution are summarized in Table 6.11.

\subsubsection{Angular Resolution}

The uncertainty on the event direction is evaluated with $\mathrm{MC}$ events by comparing the reconstructed direction to the true direction. The distribution of the cosine of the angle between the reconstructed and true event direction predicted by the $\mathrm{MC}$ is then fitted with the function [67]:

$$
R(\theta)=N\left(\mathrm{e}^{\beta_{S}(\cos \theta-1)}+\alpha_{M} \mathrm{e}^{\beta_{M}(\cos \theta-1)}\right),
$$


where $N$ is a normalization factor; $\beta_{S}$ and $\beta_{M}$ are parameters for the distribution of electrons scattered by a small and large amount, respectively; and $\alpha_{M}$ is the relative weight for the large scattering component. The uncertainty on the angular resolution is provided by the uncertainty on the $\beta_{S}$ parameter. It is an uncertainty mainly relevant for ES events.

\subsubsection{Radial Energy Bias}

The difference in observed kinetic energies $T$ between the ${ }^{16} \mathrm{~N}$ data and Monte Carlo (MC) simulation are found to change with the radial position of the ${ }^{16} \mathrm{~N}$ source inside the detector. The study of this bias during the salt phase [66] shows that the observed kinetic energy, $T$, of the electron reconstructed inside the fiducial volume depends on the radius of the event position as:

$$
T(r)=T \times\left(1.0-0.01 \frac{r}{r_{0}}\right)
$$

where $r_{0}=550 \mathrm{~cm}$. The scaling factor $T(r)$ for the radial energy bias leads to a systematic uncertainty for the SNO-II data set that is given in Table 6.11.

\subsubsection{Reconstructed Energy Dependance}

The uncertainty on the vertex accuracy affects the total number of events reconstructed inside the fiducial volume $(r<550 \mathrm{~cm})$. Any energy dependent bias, that is introduced by the pattern recognition, can potentially affect the overall shape of the CC electron energy spectrum used in the oscillation analyses. As a consequence of this, the observed kinetic energy, $T$, of the electron reconstructed 
inside the fiducial volume should by corrected by the factor [66]:

$$
\delta T=(0.31 \pm \alpha) \times(T-5.05)(\text { in } \%),
$$

where $\alpha$ is a $1 \sigma$ uncertainty in the reconstructed energy dependance systematic.

\subsubsection{Diurnal Effects}

The matter-induced neutrino oscillation (MSW effect) predicts more electron neutrino events at SNO during the night due to their regeneration inside the Earth. Any variation of the detector response between day versus night or with respect to the direction of an event; or any time variations can cause false day-night asymmetry at SNO. Diurnal energy scale and vertex accuracy uncertainties are used in the analyses of SNO-I and SNO-II data sets. In addition, diurnal uncertainty on isotropy is used with the SNO-II data set. These systematic errors are not used with the SNO-III data set because the measurement consists of total integrated fluxes, without separate samples for day and night.

\subsubsection{NCD Array}

The systematic uncertainties associated with the NCD array for the SNO-III data set are not used directly in this thesis to propagate their effects on the model predictions for the neutrino yields detected by SNO. The reason for this, as mentioned earlier, is due to the fact that we are testing the fluxes from the third phase of SNO, not the number of events. However, we use the relative errors (in \%) on the neutrino fluxes due to the systematic uncertainties as obtained by the MCMC signal extraction. The main uncertainties for the NCD array are: NCD energy scale, 
NCD energy resolution, overall neutron capture efficiency, neutron background rate and several uncertainties associated with the NCD MC simulation.

The differences between neutron calibration data of the NCD system and MC simulated data has been used to identify some of the main systematic uncertainties affecting the NCD array [68]. The first class of uncertainties originates from the physics simulation of the ionization tracks of $\alpha$ particles as well as the energy deposited by protons, alphas and tritons in the NCD counters. That class of uncertainties are referred to as $\alpha$ systematic errors. The second class of systematic errors, denoted here as instrumentals are related to the electronic and data acquisition systems.

The list of major systematics, which are related to the SNO-III data set, for both the PMT and NCD systems, including backgrounds, is shown in Table 6.12.

\subsubsection{Correlation between the SNO Systematics}

There are two types of correlation between the CC, ES and NC fluxes due to systematic errors that are considered in our analyses. First, correlation between the fluxes in a given phase that are caused by how the systematic uncertainties are evaluated. Each systematic uncertainty that affects different types of signals within a phase of SNO is assumed to be $\pm 100 \%$ correlated or null. The intraphase correlations caused by systematic uncertainties that affect different types of signals are shown in Tables 6.13 and 6.14 for the SNO-II and SNO-III data sets, respectively. Since the summed energy spectra are fitted from the SNO-I data set, these type of correlations are not considered in the analyses.

The second type of correlations are inter-phase correlations between the different phases of the experiment. The systematic errors that are considered to 
cause correlation between the three phases of SNO are: PMT energy scale, PMT energy resolution, neutron capture efficiency and neutron backgrounds. In order to estimate the correlation, each of these systematic errors has to be broken down to its individual components. The next step is to identify which of the individual contributions can be considered as fully correlated between the various phases of the experiment. Each systematic error $s_{k}$ for a particular phase $i$ is then expressed as a quadratic sum of the correlated $c_{k}$ and uncorrelated $u_{k}$ contributions:

$$
\left(s_{k}^{i}\right)^{2}=\left(c_{k}^{i}\right)^{2}+\left(u_{k}^{i}\right)^{2} .
$$

For a source of systematic error $k$, the correlation coefficient $\rho_{i-j}^{k}$ between the phases $i$ and $j$ is derived as:

$$
\rho_{i-j}^{k}=\prod_{l=i, j} \frac{c_{k}^{l}}{s_{k}^{l}}
$$

The energy scale and resolution systematic uncertainties for the PMT system are measured by SNO using the calibration data from ${ }^{16} \mathrm{~N}$ source deployed in the detector. Among all sources of energy scale and resolution uncertainties (see Table 6.15), the detector asymmetry and ${ }^{16} \mathrm{~N}$ source modeling are considered to be fully correlated between the three phases. Under this consideration, the correlation coefficients for these two uncertainties, which are shown in Table 6.18, are estimated to be $+0.54(+0.55),+0.57(0)$ and $+0.61(0)$ between the SNO-I, SNO-II and SNO-III phases for the energy scale (resolution).

The neutron capture efficiencies have been derived by using the same fission ${ }^{252} \mathrm{Cf}$ point source during all three phases of SNO. An isotropic neutron source created by mixing ${ }^{24} \mathrm{Na}$ into the heavy water has also been used during the NCD phase. The correlation coefficient $\rho_{I-I I}^{\mathrm{NC} \mathrm{C}_{\text {ef }}}$ for the neutron detection efficiency be- 
tween SNO-I and SNO-II phases are taken from Reference [69] and listed in Table 6.18. Due to the fact that during the SNO-III phase the ${ }^{252} \mathrm{Cf}$ source is used to study temporal stability and electronic gain of the NCD array, the values for the correlation coefficients between the SNO-III phase and the previous two phases are constrained to be equal to $\rho_{I-I I}^{\mathrm{NC} \text { eff }}[70]$.

The correlation for neutron backgrounds between the SNO-I and SNO-II phases are taken from Reference [69]. In the estimation of the correlation coefficients with the addition of the SNO-III data sample, only the PMT fraction of the NC background is considered to be correlated with previous phases. The NC flux from the SNO-III phase is extracted by constraining the NC fluxes measured by the PMT and NCD arrays to be the same. In terms of number of extracted NC signal events, the PMT data contribute to the total number of NC events (PMT + NCD) with a weight of about $20 \%$ [51], therefore we have taken this weight to derive the correlation coefficients for the NC backgrounds between the SNO-III and other two phases of SNO. 
Table 6.10: Systematic uncertainties for the SNO-I data set used in the solar neutrino oscillation analyses. The uncertainties are relative and quoted in percent, except for vertex resolution which is quoted in $\mathrm{cm}$.

\begin{tabular}{lc}
\hline \hline Systematics & $1 \sigma$ value \\
\hline Energy scale & $\pm 1.21 \%$ \\
Energy resolution & $\pm 4.5 \%$ \\
Energy non-linearity & $\pm 0.23 \%$ \\
Vertex resolution & $\pm 2 \mathrm{~cm}$ \\
Vertex accuracy & $\pm 1.0 \%$ \\
Diurnal Energy scale & $\pm 0.14 \%$ \\
Diurnal Vertex Accuracy & $\pm 0.2 \%$ \\
Data sacrifice & $\pm 0.3 \%$ \\
Neutron Capture & $\pm 0.99 \%$ \\
NC backgrounds day & $\pm 15.48 \%$ \\
NC backgrounds night & $\pm 15.51 \%$ \\
LEB day & $\pm 33.64 \%$ \\
LEB night & $\pm 32.93 \%$ \\
\hline \hline
\end{tabular}


Table 6.11: Systematic uncertainties for the SNO-II data set used in the solar neutrino oscillation analyses. Backgrounds are given in number of events with their uncertainties and vertex resolution in $\mathrm{cm}$, while other uncertainties are relative and quoted in percent.

\begin{tabular}{lc}
\hline \hline Systematics & $1 \sigma$ value \\
\hline Energy scale & $\pm 1.15 \%$ \\
Energy resolution & $\pm 3.4 \%$ \\
Energy non-linearity & $\pm 0.09 \%$ \\
Radial Energy Bias & $\pm 0.45 \%$ \\
Reconstructed Energy Dependance & $\pm 0.31 \%$ \\
Isotropy Mean & $\pm 0.85 \%$ \\
Isotropy Width & $\pm 0.95 \%$ \\
Vertex resolution & $\pm 2 \mathrm{~cm}$ \\
Vertex accuracy & $\pm 1.0 \%$ \\
Angular resolution & $\pm 16 \%$ \\
Diurnal Energy scale & $\pm 0.4 \%$ \\
Diurnal Vertex Accuracy & $\pm 0.3 \%$ \\
Diurnal Isotropy & $\pm 0.2 \%$ \\
Diurnal Sacrifice CC & $\pm\left(0.57_{-0.11}^{+0.16}\right) \%$ \\
Diurnal Sacrifice ES & $\pm\left(0.68_{-0.11}^{+0.16}\right) \%$ \\
Diurnal Sacrifice NC & $\pm\left(0.86_{-0.17}^{+0.21}\right) \%$ \\
Neutron Capture & $\pm\left(0.5_{-0.8}^{+0.9}\right) \%$ \\
NC Backgrounds internal & $125.1_{-32.0}^{+37.3}$ \\
NC Backgrounds external & $128.5 \pm 42.4$ \\
Low energy backgrounds combined & $6.8_{-4.5}^{+4.7}$ \\
\hline \hline
\end{tabular}


Table 6.12: Systematic uncertainties for the SNO-III data set used in the solar neutrino oscillation analyses. Backgrounds are given in number of events with their uncertainties and vertex resolution in $\mathrm{cm}$, while other uncertainties are relative and quoted in percent.

\begin{tabular}{lcc}
\hline \hline Systematics & PMT system & NCD system \\
\hline Energy scale & $\pm 1.09 \%$ & $\pm 1 \%$ \\
Energy resolution & $\pm 1.04 \%$ & $\left({ }_{-0.1}^{+0.0}\right) \%$ \\
Radial energy bias & $\pm 0.45 \%$ & - \\
Angular resolution (ES) & $\pm 6 \%$ & - \\
Vertex resolution & $\pm 3.5 \mathrm{~cm}$ & - \\
Vertex accuracy & $\pm 0.9 \%$ & - \\
Neutron capture & $1.24 \%$ & $3.32 \%$ \\
NC background internal & $56.4_{-5.4}^{+5.6}$ & $144.6_{-14.8}^{+13.8}$ \\
NC background external & $20.6 \pm 10.4$ & $40.9 \pm 20.6$ \\
Low energy backgrounds & $5.8_{-2.9}^{+9.7}$ & - \\
NCD instrumentals & - & $\pm 3 \%$ \\
NCD $\alpha$ systematics & - & $\pm 3.5 \%$ \\
PMT data sacrifice & $<1 \%$ & - \\
\hline \hline
\end{tabular}


Table 6.13: Correlation coefficients for the same sources of systematic uncertainties affecting different types of signals for the SNO-II data set.

\begin{tabular}{lccc}
\hline \hline Source of systematic & NC-CC & NC-ES & CC-ES \\
\hline Energy scale & +1 & +1 & +1 \\
Energy resolution & +1 & +1 & +1 \\
Energy non-linearity & +1 & +1 & +1 \\
Radial Energy Bias & +1 & +1 & +1 \\
Isotropy Mean & -1 & -1 & +1 \\
Isotropy Width & +1 & +1 & +1 \\
Vertex resolution & +1 & +1 & +1 \\
Vertex accuracy & +1 & +1 & +1 \\
Angular resolution & +1 & -1 & -1 \\
Neutron Capture & 0 & 0 & 0 \\
NC Backgrounds & 0 & 0 & 0 \\
Low energy backgrounds & +1 & +1 & +1 \\
\hline \hline
\end{tabular}

Table 6.14: Correlation coefficients for the same sources of systematic uncertainties affecting different types of signals for the SNO-III data set.

\begin{tabular}{lccc}
\hline \hline Source of systematic & NC-CC & NC-ES & CC-ES \\
\hline PMT energy scale & +1 & +1 & +1 \\
PMT energy resolution & +1 & +1 & +1 \\
Radial Energy Bias & +1 & +1 & +1 \\
Vertex resolution & +1 & +1 & +1 \\
Vertex accuracy & +1 & +1 & +1 \\
Angular resolution & +1 & -1 & -1 \\
Neutron Capture & +1 & +1 & +1 \\
NC Backgrounds & +1 & +1 & +1 \\
Low energy backgrounds & +1 & +1 & +1 \\
NC backgrounds & +1 & +1 & +1 \\
NCD energy scale & +1 & +1 & +1 \\
NCD energy resolution & +1 & +1 & +1 \\
NCD instrumentals & +1 & +1 & +1 \\
NCD $\alpha$ systematics & +1 & +1 & +1 \\
PMT data sacrifice & 0 & 0 & +1 \\
\hline \hline
\end{tabular}


Table 6.15: Breakdown of the individual components for the systematic uncertainties on energy scale and resolution for the three phases of SNO. The uncertainties are obtained from References $[48,65,71]$. The energy resolution components are given for neutrons; in addition they are listed for electrons in parentheses for the SNO-III data set.

\begin{tabular}{lccc}
\hline \hline \multicolumn{4}{c}{ Energy scale uncertainty } \\
\hline $\begin{array}{l}\text { Source } \\
\text { of uncertainty }\end{array}$ & SNO-I & $\begin{array}{c}\text { SNO-II } \\
\text { value }\end{array}$ & SNO-III \\
\hline Detector PMT status & - & $0.20 \%$ & $0.03 \%$ \\
Electronics threshold & - & $0.20 \%$ & $0.37 \%$ \\
Electronics gain & $0.28 \%$ & $0.40 \%$ & $0.13 \%$ \\
Electronics rate effects & $0.39 \%$ & $0.10 \%$ & $0.20 \%$ \\
Time calibration & $0.5 \%$ & $0.10 \%$ & $0.10 \%$ \\
Time drift/stability: data-MC & $0.25 \%$ & $0.15 \%$ & $0.40 \%$ \\
Radial distribution: data-MC & - & $0.45 \%$ & $0.14 \%$ \\
Detector asymmetry & $0.72 \%$ & $0.59 \%$ & $0.62 \%$ \\
16N source modeling & $0.46 \%$ & $0.65 \%$ & $0.65 \%$ \\
Cross-talk/pickup non-linearity & $0.45 \%$ & $0.25 \%$ & - \\
\hline Total & $1.21 \%$ & $1.15 \%$ & $1.09 \%$ \\
\hline \multicolumn{1}{c}{ Resolution uncertainty for neutron (electron) } \\
\hline Source SNO-I & SNO-II & SNO-III \\
of uncertainty & \multicolumn{4}{c}{ value } \\
\hline $16 \mathrm{~N}$ data-MC & $2.5 \%$ & $1.8 \%$ & $1.19(1.62) \%$ \\
Detector asymmetry & $2 \%$ & $1.4 \%$ & - \\
Radial dependence & - & $0.8 \%$ & - \\
Spacial variation & - & - & $1.04(1.41) \%$ \\
\hline Total & $4.5 \%$ & $3.4 \%$ & $2.23(3.03) \%$ \\
\hline \hline
\end{tabular}

Table 6.16: Systematic uncertainties on the neutron efficiency measurement from the calibration data in the pure $\mathrm{D}_{2} \mathrm{O}$ phase taken from [65].

\begin{tabular}{lc}
\hline \hline Source & Value in $\%$ \\
\hline Energy distribution & \pm 1.74 \\
Source position & \pm 0.95 \\
Source standard & \pm 2.20 \\
Source exclusion & \pm 0.86 \\
\hline Total uncertainty & \pm 3.09 \\
\hline \hline
\end{tabular}


Table 6.17: Systematic uncertainties on the neutron efficiency measurement from the calibration data in the salt phase taken from [48].

\begin{tabular}{lc}
\hline \hline Source & Value in $\%$ \\
\hline Source strength & \pm 0.5 \\
Source position & $+1.7,-1.0$ \\
Gamma fraction & $+0.56,-1.05$ \\
$\mathrm{AV}$ position & \pm 0.3 \\
${ }^{2} \mathrm{H}(\mathrm{n}, 2 \mathrm{n})^{1} \mathrm{H}$ & \pm 0.10 \\
${ }^{16} \mathrm{O}(\mathrm{n}, \alpha)^{13} \mathrm{C}$ & \pm 0.13 \\
Empirical fit - polynomial fit & +0.4 \\
Source sampling & \pm 1.0 \\
Source geometry & \pm 0.53 \\
\hline Total uncertainty & $+2.3,-2.0$ \\
\hline \hline
\end{tabular}

Table 6.18: Correlation coefficients for the systematic uncertainties partially correlated between the SNO-I, SNO-II and SNO-III phases.

\begin{tabular}{lccc}
\hline \hline Source of uncertainty & $\rho_{I-I I}$ & $\rho_{I-I I I}$ & $\rho_{I I-I I I}$ \\
\hline PMT energy scale & +0.54 & +0.57 & +0.61 \\
PMT energy resolution & +0.55 & 0 & 0 \\
NC detection efficiency & +0.34 & +0.40 & +0.34 \\
NC backgrounds & +0.41 & +0.08 & +0.07 \\
\hline \hline
\end{tabular}




\section{Chapter 7}

\section{Results - Two-Active Neutrino}

\section{Model}

The SNO and global analyses of solar neutrino data, under the assumption of twoneutrino oscillation hypothesis, are presented in this chapter. The details of the extraction of the neutrino mixing parameters and the methodology used to place the constrains on the allowed regions in the $\tan ^{2} \theta-\Delta m^{2}$ plane will be reviewed.

\subsection{Experimental Data}

The solar neutrino data used in the analyses are: SNO-I (pure $\mathrm{D}_{2} \mathrm{O}$ phase) summed kinetic energy spectra (CC+ES+NC+backgrounds) for day and night [64], SNOII (salt phase) CC kinetic energy spectra, ES and NC fluxes for day and night [48], SNO-III (NCD phase) CC, ES and NC fluxes [51], Super-Kamiokande zenith binned energy spectra [13,72]; and the rate measurements from the Homestake [9], Gallex/GNO [10], SAGE [44] and Borexino experiments [47]. 


\subsection{Two-Neutrino Mixing Model Predictions}

The two-neutrino mixing model, which is described in Chapter 2, was used to compute the survival probabilities $P_{e e}$ for the electron neutrinos produced in the solar core, at the positions of various detectors at the Earth. These survival probabilities are needed to infer the expected neutrino yields for the experiments used in our analyses.

\subsubsection{Solar Neutrino Survival Probabilities}

Since the analysis of the partial salt phase data set [73], SNO uses $P_{e e}$ computed numerically [74], whereas pure analytical approximations were employed in the past. The two-neutrino system evolution equation is solved for eight different neutrino sources inside the Sun: $p p$, pep, hep, ${ }^{8} \mathrm{~B},{ }^{7} \mathrm{Be},{ }^{13} \mathrm{~N},{ }^{15} \mathrm{O}$ and ${ }^{17} \mathrm{~F}$, where the radial distributions for both these neutrino sources (depicted in Figure 7.1) and solar electron density were taken from the SSM. After reaching the surface of the Sun, neutrinos are propagated through the vacuum, where due to the loss of coherence between different neutrino mass eigenstates, two types of numerical averaging of $P_{e e}$ are performed. For the squared mass differences $\left(10^{-13} \leq \Delta m^{2} / E_{\nu}<10^{-7}\right) \mathrm{eV}^{2} / \mathrm{MeV}$, the annual averaging due to the varying Earth-Sun distance is performed. For $\left(10^{-7} \leq \Delta m^{2} / E_{\nu} \leq 10^{-2}\right) \mathrm{eV}^{2} / \mathrm{MeV}$ region, $P_{e e}$ is averaged over the distance of the two oscillation lengths, which represents one of the possible ways to perform phase averaging ${ }^{1}$.

After passing into vacuum, neutrinos reach the Earth and traverse its interior along various trajectories, and finally reach a particular detector, whose precise

\footnotetext{
${ }^{1} \mathrm{~A}$ different choice for phase averaging is presented in Chapter 8 .
} 


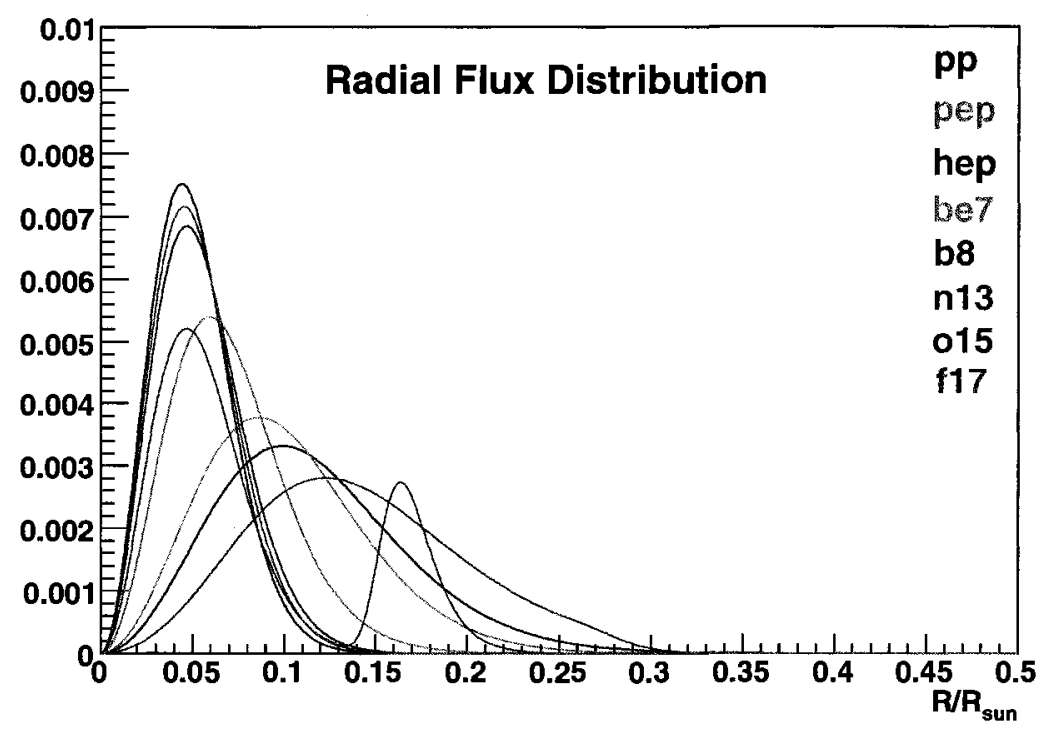

Figure 7.1: Radial distribution of the neutrino production for each of the eight different neutrino sources inside the Sun for SSM BS05(OP). View this figure in colour.

position and binned zenith livetime information must be known (c.f. Chapter 5 for SNO-III). Following the work from Baltz and Weisner [75,76], the neutrino survival probability from the Sun and vacuum, $\bar{P}_{e e}^{\odot}$, was combined with the survival probabilities $P_{e 1}^{\ominus}$ and $P_{e 2}^{\ominus}$. Here $P_{e 1}^{\ominus}$ is the probability for a pure electron neutrino state at the surface of the Earth to remain in the same state after passing through some amount of matter inside the Earth, while $P_{e 2}^{\ominus}$ is the probability for an equal mixture of electron and non-electron active neutrino states to be detected in the electron neutrino state after propagating through the Earth. The survival probabilities $P_{e 1}^{\ominus}$ and $P_{e 2}^{\ominus}$ must be determined for each detector separately and properly weighted with the experiment's zenith livetime. The final survival probability at 
the position of a detector $P_{e e}^{\oplus}$ is then given by $[75,76]$ :

$$
P_{e e}^{\oplus}=1+2 \bar{P}_{e e}^{\odot} P_{e 1}^{\ominus}-\bar{P}_{e e}^{\odot}-P_{e 1}^{\ominus}-\frac{1}{2}\left(2 \bar{P}_{e e}^{\odot}-1\right)\left(2 P_{e 2}^{\ominus}-1\right) \tan 2 \theta .
$$

\subsubsection{The Expected Neutrino Yields}

In order to calculate the model prediction for the neutrino yield at a given detector, each of the neutrino fluxes, that a particular experiment is sensitive to, is weighted with the neutrino survival probabilities $P_{e e}^{\oplus}\left(E_{\nu}\right)$, convolved with the cross-sections for the neutrino-target interactions as well as with the detector response function; and then integrated above the experiment's energy threshold. For simplicity, $P_{e e}^{\oplus}\left(E_{\nu}\right)$ will be denoted as $P_{e e}$ in the remainder of this section.

In general, the total neutrino yield for a given experiment with a neutrino energy threshold $E_{\text {th }}(\nu)$ is given by:

$$
Y=\sum_{i} \int_{E_{\mathrm{th}}}^{\infty} \phi_{i}\left(E_{\nu}\right)\left[P_{e e} \sigma_{\nu_{e} e}\left(E_{\nu}\right)+\left(1-P_{e e}\right) \sigma_{\nu_{a} e}\left(E_{\nu}\right)\right] N^{\mathrm{TF}} d E_{\nu}
$$

where $\phi_{i}\left(E_{\nu}\right)$ is a flux of neutrinos, with energy $E_{\nu}$, that originate from one of the eight production sources $\left(i=p p\right.$, pep, hep $,{ }^{7} \mathrm{Be},{ }^{8} \mathrm{~B},{ }^{13} \mathrm{~N},{ }^{15} \mathrm{O}$ and $\left.{ }^{17} \mathrm{~F}\right)$ inside the Sun. Neutrino interaction cross-sections are denoted as $\sigma_{\nu_{e}}\left(E_{\nu}\right)$ and $\sigma_{\nu_{a}}\left(E_{\nu}\right)$ for an electron and an active flavour, respectively; and $N^{\mathrm{TF}}$ is the target factor. The target factor is related to the number of targets in the interaction medium, $n^{\text {tg }}$, and to the duration (referred to as the livetime $t_{\text {live }}$ ) of exposure of the detector to the neutrino fluxes:

$$
N^{\mathrm{TF}}=n^{\mathrm{tg}} t_{\text {live }}
$$


The calculation of the number of reconstructed events then requires a good understanding of the experimental apparatus. For each solar neutrino experiment, the detector response has to be considered for the calculation of the predicted signal events. As it goes beyond the scope of this thesis, the details can be found in publications by Homestake [9], Gallex/GNO [10], SAGE [11], SK [13,72] and Borexino $[47,77]$. Here, the focus will be on the SNO experiment.

A number of the predicted $\mathrm{CC}$ events at SNO in the observed electron kinetic energy bin defined by the range between $T_{a}$ and $T_{b}$ is integrated in the following way:

$$
Y_{j}^{\mathrm{CC}}(T)=\int_{T_{a}}^{T_{b}} d T \int_{0}^{\infty} d E_{\nu} \int_{0}^{\infty} \phi\left(E_{\nu}\right) P_{e e} \frac{d \sigma_{\nu d}^{\mathrm{CC}}}{d T_{e}}\left(E_{\nu}, T_{e}\right) R_{e}\left(T, T_{e}\right) N^{\mathrm{TF}, \mathrm{d}} d T_{e}
$$

where the electron true recoil and observed kinetic energies are denoted as $T_{e}$ and $T$, respectively; while $E_{\nu}$ is the neutrino energy, $d \sigma_{\nu d}^{\mathrm{CC}} / d T_{e}$ is the differential cross-section for the CC interaction, $R_{e}\left(T, T_{e}\right)$ is the PMT response function for electrons and $N^{\mathrm{TF}, \mathrm{d}}$ is the deuteron target factor for SNO.

The number of deuteron and electrons for 1000 tonnes of heavy water at SNO, before and after the NCD array has been installed in the detector, are tabulated in Table 7.1. When the NCD array was inserted in the AV it displaced 852 liters of heavy water from the AV volume. The neutrino-deuteron differential cross-section $d \sigma_{\nu d}^{\mathrm{CC}} / d T_{e}$ matches the NSA+ (Nakamura-Sato-Ando and others) calculations from Nakamura et al. [78]. The radiative corrections on the crosssection used by SNO are described by an analytical function and depend on the 
total observed electron energy $E=m_{e}+T_{e}$ :

$$
\omega_{\mathrm{CC}}=1.0318-7.45 \times 10^{-4} E+4.72 \times 10^{-6} E^{2} .
$$

The model predictions for the number of events originated from the ES interaction at SNO and SK experiments are calculated by summing the integral yields from the electron and non-electron active neutrino fluxes contributions: $Y_{j}^{\mathrm{ES}}(T)=Y_{j}^{\mathrm{ES}, \nu_{\mathrm{e}}}(T)+Y_{j}^{\mathrm{ES}, \nu_{\mathrm{a}}}(T)$. The part of the yield originating from the elastic scattering of electron neutrinos is given by:

$$
Y_{j}^{\mathrm{ES}, \nu_{\mathrm{e}}}(T)=\int_{T_{a}}^{T_{b}} d T \int_{0}^{\infty} d E_{\nu} \int_{0}^{\infty} \phi\left(E_{\nu}\right) P_{e e} \frac{d \sigma_{\nu_{e} e}^{\mathrm{ES}}}{d T_{e}}\left(E_{\nu}, T_{e}\right) R_{e}\left(T, T_{e}\right) N^{\mathrm{TF}, \mathrm{e}} d T_{e},
$$

while the non-electron neutrino interactions yield the following number of events:

$$
Y_{j}^{\mathrm{ES}, \nu_{\mathrm{a}}}(T)=\int_{T_{a}}^{T_{b}} d T \int_{0}^{\infty} d E_{\nu} \int_{0}^{\infty} \phi\left(E_{\nu}\right)\left(1-P_{e e}\right) \frac{d \sigma_{\nu_{e}}^{\mathrm{ES}}}{d T_{e}}\left(E_{\nu}, T_{e}\right) R_{e}\left(T, T_{e}\right) N^{\mathrm{TF}, \mathrm{e}} d T_{e}
$$

The expected number of neutrino events $Y^{\mathrm{ES}, \nu_{\mathrm{e}}}$ due to the elastic scattering of electron neutrinos, that exchange both $W^{-}$and $Z^{0}$ bosons with electrons, is approximately six times higher than the number of events $Y^{\mathrm{ES}, \nu_{\mathrm{a}}}$ due to the ES interaction of other active neutrino states mediated by $Z^{0}$ bosons only. The differential cross-sections $d \sigma_{\nu, a, e}^{\mathrm{ES}} / d T_{e}$ is computed by [79]:

$$
\frac{d \sigma_{\nu_{e, a} e}^{\mathrm{ES}}}{d T_{e}}=\frac{2 G_{\mathrm{F}}^{2} m_{e}}{\pi}\left[g_{\mathrm{L}}^{2}+g_{\mathrm{R}}^{2}\left(1-\frac{T_{e}}{E_{\nu}}\right)^{2}-g_{\mathrm{L}} g_{\mathrm{R}} \frac{m_{e} T_{e}}{E_{\nu}^{2}}\right]
$$


The factors $g_{\mathrm{L}, \mathbf{R}}$ are given by:

$$
\begin{aligned}
& g_{\mathrm{L}}=\sin ^{2} \theta_{\mathrm{W}} \pm \frac{1}{2}, \\
& g_{\mathrm{R}}=\sin ^{2} \theta_{\mathrm{W}},
\end{aligned}
$$

where plus and minus signs for $g_{\mathrm{L}}$ correspond to the electron and non-electron neutrino flavours, respectively. The values of the physical constants used by SNO are: $G_{\mathrm{F}}=1.16639 \times 10^{-5} \mathrm{GeV}^{-2}, m_{e}=0.510999 \mathrm{MeV}, \sin ^{2} \theta_{\mathrm{W}}=0.23117$ and $\hbar c=197.32696 \mathrm{MeV} \mathrm{fm}$. The radiative corrections to the cross-section for the elastic scattering are implemented following the method of Reference [80]:

$$
\omega_{E S}=0.9764-7.81 \times 10^{-4} T_{e}-1.31 \times 10^{-4} T_{e}^{2}+3.64 \times 10^{-6} T_{e}^{3} .
$$

The calculation of the integral yields from the $\mathrm{NC}$ reaction at $\mathrm{SNO}$ is less complex because there is no integration over the differential cross-section:

$$
Y_{j}^{\mathrm{NC}}(T)=\int_{T_{a}}^{T_{b}} d T \int_{0}^{\infty} \phi\left(E_{\nu}\right) \sigma_{\nu d}^{\mathrm{NC}}\left(E_{\nu}\right) R_{n}(T) N^{\mathrm{TF}, \mathrm{d}} \epsilon_{e f f}^{\mathrm{NC}} d E_{\nu}
$$

where $\epsilon_{\text {eff }}^{\mathrm{NC}}$ is the neutron capture and detection efficiency. The cross-section for $\mathrm{NC}$ interaction is consistent with the NSA+ calculation with the corresponding radiative correction factor $\omega_{N C}=1.0154$ [81].

For the SNO-I summed kinetic energy spectra model predictions, the neutralcurrent (NCBG) and low energy (LEB) background spectra, as tabulated in Tables 6.2 and 6.3 , must be added to the sum of expected yields $Y^{\mathrm{CC}}+Y^{\mathrm{NC}}+Y^{\mathrm{ES}}$ for a given set of the mixing parameters $\Delta m^{2}$ and $\tan ^{2} \theta$. Background events do not need to be considered in the calculation of the SNO-II and SNO-III model 
Table 7.1: The number of electron and deuteron targets per unit volume in the SNO detector before and after the installation of the NCD array. These values are taken from Reference [81].

\begin{tabular}{lcc}
\hline \hline Number of targets in $10^{31}$ & Before NCDs & With NCDs \\
\hline deuteron & 6.0210 & 6.0153 \\
electron & 30.4046 & 30.1044 \\
\hline \hline
\end{tabular}

predictions. This is due to the fact that measurements from these two phases are tested against the model in the neutrino flux space, whereas the SNO-I data sample is tested using the total number of events.

The expectations for the gallium and chlorine rate experiments, calculated in solar neutrino units (SNU), are given by a simple integral:

$$
Y^{\mathrm{Ga}, \mathrm{Cl}}=\int_{0}^{\infty} \phi\left(E_{\nu}\right) P_{e e} \sigma^{\mathrm{Ga}, \mathrm{Cl}}\left(E_{\nu}\right) d E_{\nu}
$$

where the tables for the cross-sections $\sigma^{\mathrm{Ga}, \mathrm{Cl}}$ are created by using values from Bachall in Reference [43]. The rates $Y^{\mathrm{Ga}, \mathrm{Cl}}$ are multiplied by a factor $10^{36}$ in order to be expressed in units of SNU.

The model prediction for the Borexino experiment, that detects monoenergetic ${ }^{7} \mathrm{Be}$ neutrinos via ES interaction, is calculated in number of events per day per 100 tonnes of target volume:

$$
Y^{\mathrm{Bx}}=\phi\left(E_{\nu}\right)\left[P_{e e} \sigma_{\nu_{e} e}\left(E_{\nu}\right)+\left(1-P_{e e}\right) \sigma_{\nu_{a} e}\left(E_{\nu}\right)\right] N^{\mathrm{TF}}
$$




\subsection{Extraction of the Mixing Parameters}

A detailed description of the general method used in the extraction of neutrino mixing parameters from the solar neutrino data is presented in this section.

\subsubsection{Covariance $\chi^{2}$ Method}

The default approach for the physics interpretation of the solar neutrino data used in our analyses is the covariance $\chi^{2}$ method. Let us start with a series of $N$ observables $Y_{i}$ with their corresponding measurements $Y_{i}^{\exp }$ from a number of solar neutrino experiments and theoretical expectations $Y_{i}^{\text {th }}$. The general $\chi^{2}$ function quantifies the difference between the experimental data and theoretical model expectation for the observable $Y_{i}$ :

$$
\chi^{2}=\sum_{i, j=1}^{N}\left(Y_{i}^{\exp }-Y_{i}^{\mathrm{th}}\right)^{T}\left[\sigma_{i j}^{2}(\mathrm{tot})\right]^{-1}\left(Y_{j}^{\exp }-Y_{j}^{\mathrm{th}}\right) .
$$

The experimental values or data $Y_{i}^{\exp }$ for the corresponding observable $Y_{i}$ could be the total rates measured by radiochemical experiments; or the $\mathrm{CC}$, ES or NC fluxes from SNO; or one of the spectral bins from SNO day and night; or SK zenith binned spectral data; or the rate measured by Borexino. A detailed description of the data samples from SNO is presented in Chapter 6, whereas more information on the data from other experiments can be found in the corresponding publications $[9,10,13,44,47,72]$. The model prediction $Y_{i}^{\text {th }}$ is calculated under the assumption of the two-neutrino oscillation hypothesis, thus it depends on the number of free parameters $n$ in the fit. In the physics interpretation presented in this thesis, the free parameters are the neutrino mixing parameters $\left(\Delta m^{2}\right.$ and 
$\left.\tan ^{2} \theta\right)$ and the total flux of the ${ }^{8} \mathrm{~B}$ neutrinos $\phi_{8_{\mathrm{B}}}$. The shape of the ${ }^{8} \mathrm{~B}$ spectrum is fully constrained by the mixing parameters. The number of degrees of freedom (dof) is defined as a difference between the total number of observables $N$ used in the calculation and the number of free parameters $n: d o f=N-n$.

The covariance error matrix $\sigma_{i j}^{2}$ (tot) is built as a sum of the squares of the total experimental $\sigma_{i j}^{2}(\exp )$ and theoretical $\sigma_{i j}^{2}($ th $)$ uncertainties:

$$
\sigma_{i j}^{2}(\mathrm{tot})=\sigma_{i j}^{2}(\exp )+\sigma_{i j}^{2}(\mathrm{th})
$$

where the experimental errors are composed of statistical and systematic contributions from each experiment:

$$
\sigma_{i j}^{2}(\exp )=\sigma_{i j}^{2}(\text { stat })+\sigma_{i j}^{2}(\text { syst })
$$

Observables from a particular experiment could be correlated, which is the case for the SNO-II and SNO-III data sets, thus the general definition of the statistical covariance matrix is given by:

$$
\sigma_{i j}^{2}(\text { stat })=\rho_{i j} u_{i} u_{j}
$$

where $u_{i}$ and $\rho_{i j}$ are the statistical error for the measurement $Y_{i}^{\exp }$ and statistical correlation coefficient between the observables $Y_{i}^{\exp }$ and $Y_{j}^{\exp }$, respectively. The statistical correlation coefficients are tabulated in Tables 6.8 and 6.9 for the SNOII day and night data samples, and in Table 6.7 for the SNO-III averaged fluxes.

The total systematic uncertainty on each radiochemical, and also the Borexino rate measurement, is uncorrelated with experimental systematic uncertainties 
from other experiments, thus it can be added in quadrature to the statistical error, giving rise to diagonal entries in the error matrix $\sigma_{i i}^{2}(\exp )$. The systematic error matrix $\sigma_{i j}^{2}$ (syst) for other experiments (such as SNO and SK) is discussed in Section 7.3.2.

\subsubsection{Experimental Systematic Uncertainties}

Correlated experimental systematic uncertainties can affect at the same time the different signals within one experiment (e.g. SNO CC and ES fluxes), all the spectral bins for a particular spectral measurements (e.g. SK zenith energy spectra, SNO-I summed kinetic energy spectra and SNO-II CC kinetic energy spectra) and measurements from the different phases of one experiment (e.g. the three phases of SNO).

The effect of each systematic uncertainty $S_{k}$ on the model expectation for the neutrino yield $Y_{i}^{\text {th }}$ is estimated by computing the partial derivatives of the model $Y_{i}^{\text {th }}$ with respect to the source of the uncertainty $S_{k}$ :

$$
\beta_{i k}=\frac{1}{Y_{i}^{\mathrm{th}}} \frac{\partial Y_{i}^{\mathrm{th}}}{\partial S_{k}} .
$$

The partial derivatives $\beta_{i k}$ are used to construct the systematic error matrix $\sigma_{i j}^{2}$ (syst), which is defined as:

$$
\sigma_{i j}^{2}(\mathrm{syst})=Y_{i}^{\mathrm{th}} Y_{j}^{\mathrm{th}} \sum_{k=1}^{K} r_{i j}^{k} \rho_{i j}^{k} \beta_{i k} \beta_{j k}\left(\Delta S_{k}\right)^{2}=\sum_{k=1}^{K} r_{i j}^{k} \rho_{i j}^{k} \frac{\partial Y_{i}^{\mathrm{th}}}{\partial S_{k}} \frac{\partial Y_{j}^{\mathrm{th}}}{\partial S_{k}}\left(\Delta S_{k}\right)^{2}
$$

where $\mathrm{K}$ is the number of systematic errors affecting the observables $i$ and $j$, and $\Delta S_{k}$ is the $1 \sigma$ value for the systematic uncertainty $S_{k}$. The latter are tabulated 
in Tables $6.10,6.11$ and 6.12 for SNO. A coefficient $r_{i j}^{k}$ describes a correlation between the observables $i$ and $j$ induced by the systematic error $k$, within a single phase of a given experiment. For a source of systematic error $k$, a partial correlation between the observables from different phases of a single experiment is given by the coefficient $\rho_{i j}^{k}$. Note that by construction, $r_{i j}^{k}$ are set to zero for SNO-I data sample since the analysis consists of the sum of the CC, ES and NC signals. Values of the correlation coefficients $r_{i j}^{k}$ are tabulated in Tables 6.13 and 6.14 for the SNO-II and SNO-III data samples, respectively. The correlation coefficients $\rho_{i j}^{k}$ between the three phases of SNO are given in Table 6.18.

A more sophisticated and consistent way to combine the three phases of SNO is being developed. In the context of this dissertation, the method used is adequate since correlations via the experimental systematic uncertainties for a given experiment have little impact on the resulting confidence region contours in the neutrino mixing parameter space [82].

\subsubsection{Calculation of the Partial Derivatives $\boldsymbol{\beta}_{i k}$}

The derivatives $\beta_{i k}$ describe the change in the expected neutrino yield $Y_{i}^{\text {th }}$ due to the effect of the source $S_{k}$ of systematic uncertainty. A method for computing the parameters $\beta_{i k}$ used in this dissertation and in the SNO publications is described here.

The model expectations for the neutrino yields depend on the neutrino oscillation parameters, thus the partial derivatives $\beta_{i k}$, ideally, should be computed at each value of $\tan ^{2} \theta$ and $\Delta m^{2}$. However, this procedure demands an extensive amount of the processing time (CPU), due to the large number of systematic errors involved in the global solar analysis. The derivatives for the dominant and most 
important systematic uncertainties, such as energy scale and energy resolution for the SNO and SK experiments are calculated at each point in the $\tan ^{2} \theta-\Delta m^{2}$ plane. The rest of the partial derivatives are either computed assuming the nonoscillated SSM neutrino yield $Y_{i}^{\mathrm{SSM}}$ or directly obtained from the signal extraction as relative fractional errors on the measurements $Y_{i}^{\exp }$. For SNO, the signal extraction procedure relies on a maximum likelihood function to discriminate the signals and various backgrounds [83] or the MCMC method [60,61].

A method used to numerically derive $\beta_{i k}$ is performed by calculating perturbed model expectations for each neutrino yield $Y_{i, k}^{\mathrm{th}, \pm}$ for $\pm n \sigma$ variation due to the source of systematic uncertainty $S_{k}$. The partial derivative is then estimated as:

$$
\begin{gathered}
\beta_{i k}^{ \pm}=\frac{1}{Y_{i}^{\mathrm{th}, \pm}}\left(Y_{i, k}^{\mathrm{th}, \pm}-Y_{i, k}^{\mathrm{th}}\right), \\
\beta_{i k}=\frac{\beta_{i k}^{+}-\beta_{i k}^{-}}{n} .
\end{gathered}
$$

In the early analysis of the pure $\mathrm{D}_{2} \mathrm{O}$ data [64], SNO computed $\beta_{i k}$ derivatives non-analytically, but by smearing the SSM expectation from Monte Carlo (MC) simulation for each systematic uncertainty. The MC SSM data sample was not recomputed for each systematic error by changing the detector response parameters, but instead by finding the effect in energies, positions and directions due to the effect of a given systematic uncertainty. This is not a recommended method of computing the derivatives because only one MC data sample produced with the optimal detector response parameters was used. For the first analyses of the partial salt data set [73], a significant improvement was made in the calculation of the partial derivatives for the energy scale and energy resolution [74]. The 
first improvement was made in changing the detector response parameters and recalculating the model expectations due to the effect of these systematic uncertainties, rather than using a single set of SSM MC data. This approach is referred as an analytical method further in this section. The second, not less important, improvement came from the fact that the energy scale, energy resolution and ${ }^{8} \mathrm{~B}$ spectrum shape partial derivatives were calculated at each point in the $\tan ^{2} \theta-\Delta m^{2}$ plane.

After the 391-day salt phase of SNO, the $\beta_{i k}$ parameters were recalculated and applied consistently both to the SNO-I and SNO-II data sets by changing the detector response parameters, and by consistently recomputing the model expectations $Y_{i k}^{\mathrm{th}, \pm}$ for each source of systematic uncertainty, i.e. analytically. The same analytical approach has been adopted for the analysis of SNO-III data set, with respect to the energy scale and energy resolution systematic uncertainties.

$\boldsymbol{\beta}_{i k}$ for Energy Related Systematic Uncertainties The energy related systematic uncertainties, for which the partial derivatives are computed analytically, are: energy scale, energy resolution, energy non-linearity and reconstructed energy dependance. These systematic uncertainties directly affect the PMT response functions given by Equations (4.9), (4.11) and (4.12). In order to compute perturbed model expectations $Y^{\text {th, }, \pm}$, the detector response function has to be changed for the effect of each of these uncertainties.

For the energy scale uncertainty, the observed energy $E$ for a given event is shifted as:

$$
E \rightarrow \frac{1}{1 \pm \Delta S_{\text {escale }}} E
$$

and passed to the detector response function in terms of kinetic energy 
$T=E-m_{e}$. The new response function is used to derive the perturbed model expectations $Y_{i \text {,escale, }}^{\mathrm{th}, \pm}$, and subsequently the corresponding partial derivative. This procedure is repeated for each kinetic energy bin and at each point in the $\tan ^{2} \theta-\Delta m^{2}$ plane. This is the largest uncertainty that affect both the shape of the kinetic energy spectra and total number of extracted events at the SNO and SK experiments.

The same procedure is applied to the small diurnal energy scale uncertainty, but for a non-oscillating model prediction $\left(\tan ^{2} \theta=0\right.$ and $\left.\Delta m^{2}=0\right)$.

The uncertainty on energy resolution affects the energy resolution $\sigma_{E}$ of the detector response by shifting it:

$$
\sigma_{E}^{\prime}=\sigma_{E}\left(1 \pm \Delta \sigma_{E}\right)
$$

where $\Delta \sigma_{E}$ is related to energy resolution uncertainty as given in Equation (6.1). The new resolution function $\sigma_{E}^{\prime}$ is used in the detector response to recompute the yields $Y_{i, \text { eres }}^{\mathrm{th}, \pm}$, for each kinetic energy bin, and at each point in the $\tan ^{2} \theta-\Delta m^{2}$ plane.

This procedure is also applied to the small diurnal energy resolution uncertainty, but for a non-oscillating model prediction $\left(\tan ^{2} \theta=0\right.$ and $\left.\Delta m^{2}=0\right)$. The energy non-linearity $S_{\text {enl }}$ and reconstructed energy dependance $S_{\text {red }}$ uncertainties change the observed kinetic energy $\mathrm{T}$ of electron as:

$$
T \rightarrow \frac{1}{1 \pm \Delta S_{\mathrm{enl} / \mathrm{red}}} T+\frac{\Delta S_{\mathrm{enl} / \mathrm{red}} \times 5.05}{1+\Delta S_{\mathrm{enl} / \mathrm{red}}} .
$$

Again, the new shifted kinetic energies are used by the detector response 
function to compute the model predictions for the effect of these two systematic errors, at each energy bin and for the non-oscillating yields $\left(\tan ^{2} \theta=0\right.$ and $\left.\Delta m^{2}=0\right)$.

\section{$\boldsymbol{\beta}_{\boldsymbol{i}}$ for Energy Independent Systematic Uncertainties The partial deriva-} tives are computed analytically for some of systematic uncertainties that do not depend on energy of an event: neutron capture, vertex accuracy and sacrifice. These uncertainties linearly affect the model predictions $Y^{\text {th }}$ computed by the default detector response as:

$$
Y^{\mathrm{th}, \pm}=Y^{\mathrm{th}}\left(1 \pm \Delta S_{\mathrm{k}}\right)
$$

The only exception to this method was made in handling the vertex resolution uncertainty, which also does not depend on energy, but where the position information for each signal event was needed. This information can be obtained only from the SNO Monte Carlo that simulates the detector response, and not from the model expectation computed as a function of energy only. Hence, the MC simulation for the SSM prediction at SNO has been used. However an improvement has been made in the calculation regarding this uncertainty with respect to the former method used for the SNO analyses in [64] and [73]. The vertex resolution uncertainty is the error on the width of the vertex distribution function given in Equation (6.4), which can be approximated for the pure $\mathrm{D}_{2} \mathrm{O}$ phase as: $\sigma \simeq\left(17 \pm \Delta S_{\mathrm{vr}}\right)$ cm, with $\Delta S_{\mathrm{vr}}$ being its $1 \sigma$ error. For each event in the MC data set, its 
position coordinates $(x, y, z)$ are smeared as:

$$
r=\sqrt{(x \pm p)^{2}+(y \pm p)^{2}+(z \pm p)^{2}}
$$

where the smearing term $p$ is a random number from Gaussian distribution $G\left(0, \sigma_{\Delta}^{2}\right)$. The width $\sigma_{\Delta}$ of the Gaussian $G\left(0, \sigma_{\Delta}^{2}\right)$ is related to the width of the vertex distribution function $\sigma$ with the uncertainty $\Delta S_{\mathrm{vr}}$ by :

$$
\sigma+\Delta S_{\mathrm{vr}}=\sqrt{\sigma^{2}+\sigma_{\Delta}^{2}}
$$

Hence, $\sigma_{\Delta}$ is given by:

$$
\sigma_{\Delta}=\sqrt{\Delta S_{\mathrm{vr}}^{2}+2 \sigma \Delta S_{\mathrm{vr}}}
$$

The smearing factor $p$ for each coordinate has been sampled from 10000 random numbers to assure a reliable distribution, rather than using just a single random number as in previous SNO analysis. With the new smeared coordinates and after applying positional $(r<550 \mathrm{~cm})$, directional and energy cuts, the perturbed expectations for neutrino yields $Y_{i, \mathrm{vr}}^{\mathrm{th}, \pm}$ are obtained and subsequently used for calculation of the partial derivatives with respect to the vertex resolution systematic uncertainty.

Relative errors on SNO-I summed spectra $\left(\Delta Y_{i} / Y_{i}=\beta_{i k} \Delta S_{k}\right)$ inferred with the partial derivatives $\beta_{i k}$ for energy non-linearity, vertex accuracy, vertex resolution, as well as diurnal effect on energy scale and vertex resolution are summarized in Tables 7.2 and 7.3 for day and night, respectively, while the relative errors for energy scale and energy resolution experimental systematic uncertainties for day 
and night, computed for $\tan ^{2} \theta=0$ and $\Delta m^{2}=0$ are tabulated in Table 7.4. The latter are shown as an example only, since in our analysis the partial derivatives for these two systematic uncertainties are recalculated at each point in the $\tan ^{2} \theta-$ $\Delta m^{2}$ space. The SNO-I analysis does not include an error due to the neutrinodeuteron cross-section.

Table 7.5 shows the relative errors $\left(\Delta Y_{i} / Y_{i}=\beta_{i k} \Delta S_{k}\right)$ on the SNO-II CC day and night spectrum, not including energy scale and resolution relative errors that are obtained by using the derivatives computed at each point in $\tan ^{2} \theta-\Delta m^{2}$ plane. The remaining relative errors (radial energy bias, vertex resolution, isotropy mean and width, angular resolution and diurnal systematic uncertainties) are provided by the signal extraction and can be found in Reference [48]. The relative errors on the SNO-II NC and ES fluxes are obtained from the signal extraction and summarized in Table 7.6, except for the energy scale and energy resolution relative errors that are recomputed analytically for each value of the neutrino mixing parameters. The errors associated with the neutrino-deuteron cross-section are taken from Reference [84].

For the analysis of the SNO-III data set, the energy scale and energy resolution $\beta_{i k}$ parameters are also calculated analytically and at each point in $\tan ^{2} \theta-\Delta m^{2}$ plane. The remaining parameters are obtained from the signal extraction as relative errors on the extracted CC, ES and NC fluxes, which are summarized in Table 7.7. The errors on the neutrino-deuteron cross-section are taken from Reference [84].

Finally, even if the relative errors (or equivalently partial derivatives) for energy related systematic uncertainties (energy scale and energy resolution) are calculated analytically at each point of the $\tan ^{2} \theta-\Delta m^{2}$ plane, we advise phenomenologists 
Table 7.2: SNO-I day relative errors $\left(\Delta Y_{i} / Y_{i}=\beta_{i k} \Delta S_{k}\right.$ in percent) computed under assumption of the non-oscillating model. The energy scale and resolution relative errors are not shown in this table because they are recomputed at each point in the $\tan ^{2} \theta-\Delta m^{2}$ plane, which is the recommended method for all energy depended systematic uncertainties. The examples of the non-oscillating relative errors for these two systematical uncertainties are tabulated in Table 7.4.

\begin{tabular}{ccccccc}
\hline \hline $\begin{array}{c}\text { Energy bin } \\
(\mathrm{MeV})\end{array}$ & $\begin{array}{c}\text { Energy } \\
\text { non-lin. }\end{array}$ & $\begin{array}{c}\text { Vertex } \\
\text { acc. }\end{array}$ & $\begin{array}{c}\text { Vertex } \\
\text { res. }\end{array}$ & $\begin{array}{c}\text { Data } \\
\text { sacrifice }\end{array}$ & $\begin{array}{c}\text { En. scale } \\
\text { diurnal }\end{array}$ & $\begin{array}{c}\text { Vertex acc. } \\
\text { diurnal }\end{array}$ \\
\hline $5.0-5.5$ & -0.41 & -2.64 & -2.30 & 0.30 & -0.21 & -0.27 \\
$5.5-6.0$ & -0.24 & -2.47 & -0.12 & 0.30 & 0.01 & -0.25 \\
$6.0-6.5$ & -0.26 & -2.70 & 0.04 & 0.30 & 0.03 & -0.27 \\
$6.5-7.0$ & -0.23 & -2.82 & 0.02 & 0.30 & -0.01 & -0.26 \\
$7.0-7.5$ & -0.15 & -2.76 & 0.06 & 0.30 & 0.01 & -0.27 \\
$7.5-8.0$ & -0.18 & -2.58 & 0.22 & 0.30 & 0.11 & -0.25 \\
$8.0-8.5$ & -0.03 & -2.85 & 0.28 & 0.30 & 0.01 & -0.23 \\
$8.5-9.0$ & 0.05 & -2.81 & 0.04 & 0.30 & 0.17 & -0.28 \\
$9.0-9.5$ & 0.10 & -3.05 & 0.10 & 0.30 & 0.18 & -0.28 \\
$9.5-10.0$ & 0.56 & -3.07 & 0.30 & 0.30 & 0.20 & -0.38 \\
$10.0-10.5$ & 0.77 & -2.86 & 0.44 & 0.30 & 0.39 & -0.31 \\
$10.5-11.0$ & 0.51 & -3.18 & -0.06 & 0.30 & 0.43 & -0.24 \\
$11.0-11.5$ & 1.31 & -2.76 & 0.12 & 0.30 & 0.50 & -0.12 \\
$11.5-12.0$ & 2.15 & -2.46 & -0.12 & 0.30 & 0.63 & -0.29 \\
$12.0-12.5$ & 2.16 & -3.04 & -0.44 & 0.30 & 0.44 & -0.31 \\
$12.5-13.0$ & 0.97 & -2.90 & -0.26 & 0.30 & 1.09 & -0.45 \\
$13.0-20.0$ & 3.28 & -3.28 & -0.56 & 0.30 & 1.02 & -0.28 \\
\hline \hline
\end{tabular}


Table 7.3: SNO-I night relative errors $\left(\Delta Y_{i} / Y_{i}=\beta_{i k} \Delta S_{k}\right.$ in percent) computed under assumption of the non-oscillating model. The energy scale and resolution relative errors are not shown in this table because they are recomputed at each point in the $\tan ^{2} \theta-\Delta m^{2}$ plane, which is the recommended method for all energy depended systematic uncertainties. The examples of the non-oscillating relative errors for these two systematical uncertainties are tabulated in Table 7.4.

\begin{tabular}{ccccccc}
\hline \hline $\begin{array}{c}\text { Energy Bin } \\
(\mathrm{MeV})\end{array}$ & $\begin{array}{c}\text { Energy } \\
\text { non-lin. }\end{array}$ & $\begin{array}{c}\text { Vertex } \\
\text { acc. }\end{array}$ & $\begin{array}{c}\text { Vertex } \\
\text { res. }\end{array}$ & $\begin{array}{c}\text { Data } \\
\text { sacrifice }\end{array}$ & $\begin{array}{c}\text { En.scale } \\
\text { diurnal }\end{array}$ & $\begin{array}{c}\text { Vertex acc. } \\
\text { diurnal }\end{array}$ \\
\hline $5.0-5.5$ & -0.31 & -2.65 & -0.02 & 0.30 & 0.04 & 0.27 \\
$5.5-6.0$ & -0.33 & -2.62 & -0.02 & 0.30 & 0.13 & 0.22 \\
$6.0-6.5$ & -0.28 & -2.69 & -0.04 & 0.30 & -0.02 & 0.33 \\
$6.5-7.0$ & -0.14 & -2.89 & 0.16 & 0.30 & -0.07 & 0.22 \\
$7.0-7.5$ & -0.23 & -2.81 & 0.20 & 0.30 & -0.01 & 0.29 \\
$7.5-8.0$ & -0.16 & -3.01 & 0.00 & 0.30 & 0.02 & 0.28 \\
$8.0-8.5$ & -0.02 & -2.89 & -0.02 & 0.30 & -0.17 & 0.31 \\
$8.5-9.0$ & -0.30 & -2.79 & 0.11 & 0.30 & -0.05 & 0.25 \\
$9.0-9.5$ & 0.08 & -2.71 & 0.02 & 0.30 & -0.14 & 0.24 \\
$9.5-10.0$ & 0.32 & -2.88 & -0.06 & 0.30 & -0.32 & 0.30 \\
$10.0-10.5$ & 0.72 & -2.94 & 0.04 & 0.30 & -0.44 & 0.26 \\
$10.5-11.0$ & 0.84 & -2.85 & -0.06 & 0.30 & -0.37 & 0.26 \\
$11.0-11.5$ & 1.71 & -2.77 & 0.02 & 0.30 & -0.63 & 0.23 \\
$11.5-12.0$ & 0.58 & -2.42 & 0.54 & 0.30 & 0.04 & 0.24 \\
$12.0-12.5$ & 2.57 & -2.71 & -0.36 & 0.30 & -1.31 & 0.34 \\
$12.5-13.0$ & 1.13 & -2.41 & 0.40 & 0.30 & -0.49 & 0.15 \\
$13.0-20.0$ & 3.21 & -2.26 & -0.38 & 0.30 & -1.24 & 0.19 \\
\hline \hline
\end{tabular}


Table 7.4: Example of day and night SNO-I relative errors $\left(\Delta Y_{i} / Y_{i}=\beta_{i k} \Delta S_{k}\right.$ in percent) for energy scale and energy resolution systematic uncertainties, which are computed under the non-oscillating assumption. The errors associated with energy scale and energy resolution are recomputed in reality at each point in the $\tan ^{2} \theta-\Delta m^{2}$.

\begin{tabular}{ccccc}
\hline \hline Energy Bin & \multicolumn{2}{c}{ Energy scale (\%) } & \multicolumn{2}{c}{ Energy resolution (\%) } \\
$(\mathrm{MeV})$ & Day & Night & Day & Night \\
\hline $5.0-5.5$ & -1.56 & -1.48 & -0.17 & -1.31 \\
$5.5-6.0$ & -0.68 & -0.82 & -0.45 & 0.44 \\
$6.0-6.5$ & 0.02 & 0.13 & -1.20 & -0.19 \\
$6.5-7.0$ & 0.75 & 0.14 & 2.18 & -0.08 \\
$7.0-7.5$ & -0.18 & 0.44 & -1.99 & -1.17 \\
$7.5-8.0$ & 1.16 & 0.65 & -1.54 & 0.60 \\
$8.0-8.5$ & 1.17 & 1.18 & -1.49 & -1.12 \\
$8.5-9.0$ & 2.49 & 2.84 & -1.54 & -2.74 \\
$9.0-9.5$ & 2.70 & 2.97 & -1.14 & -2.76 \\
$9.5-10.0$ & 5.38 & 4.10 & -1.05 & -1.76 \\
$10.0-10.5$ & 5.03 & 5.16 & 1.79 & 1.46 \\
$10.5-11.0$ & 6.45 & 7.92 & -3.48 & 0.04 \\
$11.0-11.5$ & 8.76 & 9.40 & 2.39 & 1.26 \\
$11.5-12.0$ & 12.64 & 8.66 & 9.09 & 6.61 \\
$12.0-12.5$ & 10.04 & 12.84 & 7.92 & 1.73 \\
$12.5-13.0$ & 12.74 & 13.11 & 7.59 & 10.51 \\
$13.0-20.0$ & 19.47 & 18.74 & 25.76 & 23.38 \\
\hline \hline
\end{tabular}


Table 7.5: SNO-II day and night relative errors $\left(\Delta Y_{i} / Y_{i}=\beta_{i k} \Delta S_{k}\right.$ in percent) on $\mathrm{CC}$ spectra, which are computed under the non-oscillating assumption. The energy scale and resolution relative errors are not shown in this table because they are recomputed at each point in the $\tan ^{2} \theta-\Delta m^{2}$ plane, which is the recommended method for all energy depended systematical uncertainties. The remaining SNOII relative errors are obtained from the signal extraction and can be found in Reference [48].

\begin{tabular}{|c|c|c|c|c|}
\hline \multirow{2}{*}{$\begin{array}{c}\text { Energy Bin } \\
(\mathrm{MeV})\end{array}$} & \multicolumn{2}{|c|}{ Energy non-linearity (\%) } & \multicolumn{2}{|c|}{ Recon. En. Dependance(\%) } \\
\hline & Day & Night & Day & Night \\
\hline $5.5-6.0$ & -0.00 & -0.00 & -0.02 & -0.02 \\
\hline $6.0-6.5$ & -0.02 & -0.02 & -0.07 & -0.07 \\
\hline $6.5-7.0$ & 0.02 & 0.02 & 0.09 & 0.09 \\
\hline $7.0-7.5$ & 0.02 & 0.02 & 0.10 & 0.10 \\
\hline $7.5-8.0$ & 0.04 & 0.04 & 0.20 & 0.20 \\
\hline $8.0-8.5$ & 0.07 & 0.07 & 0.34 & 0.34 \\
\hline $8.5-9.0$ & 0.10 & 0.10 & 0.50 & 0.50 \\
\hline $9.0-9.5$ & 0.14 & 0.14 & 0.71 & 0.71 \\
\hline $9.5-10.0$ & 0.19 & 0.19 & 0.95 & 0.95 \\
\hline $10.0-10.5$ & 0.25 & 0.25 & 1.23 & 1.23 \\
\hline $10.5-11.0$ & 0.31 & 0.31 & 1.55 & 1.55 \\
\hline $11.0-11.5$ & 0.38 & 0.38 & 1.92 & 1.92 \\
\hline $11.5-12.0$ & 0.47 & 0.47 & 2.35 & 2.35 \\
\hline $12.0-12.5$ & 0.47 & 0.47 & 2.81 & 2.81 \\
\hline $12.5-13.0$ & 0.66 & 0.66 & 3.16 & 3.16 \\
\hline $13.0-13.5$ & 0.76 & 0.76 & 3.81 & 3.81 \\
\hline $13.5-20.5$ & 0.97 & 0.97 & 4.85 & 4.85 \\
\hline
\end{tabular}


Table 7.6: Relative errors $\left(\Delta Y_{i} / Y_{i}=\beta_{i k} \Delta S_{k}\right.$ in percent) on NC and ES day and night fluxes for SNO-II phase obtained from signal extraction. The energy scale and resolution parameters are not listed because they are recomputed at each point in the $\tan ^{2} \theta-\Delta m^{2}$ plane. The relative errors due to the uncertainties on the cross-section are taken from Reference [84].

\begin{tabular}{|c|c|c|c|c|}
\hline $\begin{array}{l}\text { Source of } \\
\text { uncertainty }\end{array}$ & $\begin{array}{c}\text { NC day. } \\
(\%)\end{array}$ & $\begin{array}{c}\text { NC night. } \\
(\%)\end{array}$ & $\begin{array}{c}\text { ES day } \\
(\%)\end{array}$ & $\begin{array}{c}\text { ES Night } \\
(\%)\end{array}$ \\
\hline Radial en. bias & \multicolumn{2}{|c|}{2.00} & \multicolumn{2}{|c|}{1.17} \\
\hline$\beta_{14}$ mean & \multicolumn{2}{|c|}{-3.35} & \multicolumn{2}{|c|}{1.24} \\
\hline$\beta_{14}$ width & \multirow{2}{*}{\multicolumn{2}{|c|}{$\begin{array}{l}0.25 \\
3.16\end{array}$}} & \multicolumn{2}{|c|}{0.20} \\
\hline Vertex accuracy & & & \multicolumn{2}{|c|}{2.80} \\
\hline Angular resolution & \multicolumn{2}{|c|}{0.22} & \multicolumn{2}{|c|}{-5.06} \\
\hline NC background & \multicolumn{2}{|c|}{1.74} & - & - \\
\hline Neutron capture & \multicolumn{2}{|c|}{2.52} & - & - \\
\hline Čerenkov/AV backgrounds & \multicolumn{2}{|c|}{0.95} & - & - \\
\hline Diurnal NC(ES) & 0.87 & -0.87 & 1.18 & -1.18 \\
\hline Diurnal en. scale & 1.39 & -1.39 & 0.28 & -0.28 \\
\hline Diurnal isotropy & 2.51 & -2.51 & 0.74 & -0.74 \\
\hline Diurnal vertex res. & 0.55 & -0.55 & 0.30 & -0.30 \\
\hline Cross section & 1.1 & 1.1 & - & - \\
\hline
\end{tabular}


Table 7.7: Relative errors $\left(\Delta Y_{i} / Y_{i}=\beta_{i k} \Delta S_{k}\right.$ in percent $)$ on CC, ES and NC fluxes for SNO-III phase obtained from signal extraction. The PMT energy scale and resolution parameters are not listed because they are recomputed at each point in the $\tan ^{2} \theta-\Delta m^{2}$ plane. The relative errors due to the uncertainties on the cross-section are taken from Reference [84].

\begin{tabular}{cccc}
\hline \hline $\begin{array}{c}\text { Source of } \\
\text { uncertainty }\end{array}$ & $\begin{array}{c}\text { NC uncert. } \\
(\%)\end{array}$ & $\begin{array}{c}\text { CC uncert. } \\
(\%)\end{array}$ & $\begin{array}{c}\text { ES uncert. } \\
(\%)\end{array}$ \\
\hline PMT radial scaling & 0.1 & 2.7 & 2.7 \\
PMT angular resolution & 0.0 & 0.2 & -2.2 \\
PMT radial energy dep. & 0.0 & 0.9 & 0.9 \\
Background neutrons & 2.3 & 0.6 & 0.7 \\
Neutron capture & 3.3 & 0.4 & 0.5 \\
Cerenkov/AV backgrounds & 0.0 & 0.3 & 0.3 \\
NCD instrumentals & 1.6 & 0.2 & 0.2 \\
NCD energy scale & 0.5 & 0.1 & 0.1 \\
NCD energy resolution & 2.7 & 0.3 & 0.3 \\
NCD alpha systematics & 2.7 & 0.3 & 0.4 \\
PMT data cleaning & 0.0 & 0.3 & 0.3 \\
\hline Cross section & 1.1 & 1.2 & - \\
\hline \hline
\end{tabular}

or analysts who are non-SNO collaborators to use fixed relative errors on the measurements due to these two uncertainties. This is justifiable by a small overall difference in final results from analyses using the analytical relative errors on the model that vary across the $\tan ^{2} \theta-\Delta m^{2}$ plane versus fixed relative errors obtained from the signal extraction or computed for the non-oscillated SSM yields [82].

\subsubsection{Theoretical Uncertainties}

By construction, a theoretical uncertainty on a given solar reaction is common to all experiments that are sensitive to this reaction, thus making theoretical error fully correlated between all the solar neutrino experiments. The uncertainty, which also affects all the solar neutrino data at the same time, is the error on the ${ }^{8} \mathrm{~B}$ spectrum shape. For the purpose of the analyses presented in this thesis, the ${ }^{8} \mathrm{~B}$ 
spectrum shape is obtained either from Ortiz et al. [85] with the spectrum errors derived by Bachall et al. [86], or from the latest results by Winter et al. [63]. It should be stressed that the latter uncertainty correlates all the experiments that measure the ${ }^{8} \mathrm{~B}$ neutrino flux.

The theoretical error matrix $\sigma_{i j}^{2}(\mathrm{th})$ is composed of three submatrices that account for the contributions from each rate experiment's error on the neutrino capture cross-section $\sigma_{i j}^{2}(\mathrm{cs})$, astrophysical theoretical errors $\sigma_{i j}^{2}$ (ap) that are related to the inputs from the SSM and correlate all the experiments; and the error on the ${ }^{8} \mathrm{~B}$ spectrum shape $\sigma_{i j}^{2}\left({ }^{8} \mathrm{~B}\right)$ :

$$
\sigma_{i j}^{2}(\mathrm{th})=\sigma_{i j}^{2}(\mathrm{cs})+\sigma_{i j}^{2}(\mathrm{ap})+\sigma_{i j}^{2}\left({ }^{8} \mathrm{~B}\right)
$$

The cross-sectional error components $\sigma_{i j}^{2}(\mathrm{cs})$ for the chlorine (Homestake) and gallium (Gallex/GNO and SAGE) experiments are added quadratically as:

$$
\sigma_{i j}^{2}(\mathrm{cs})=\delta_{X(i), X(j)} \sum_{k_{1}=1}^{8} \sum_{k_{2}=1}^{8} \delta_{k_{1}, k_{2}} \frac{\partial Y_{i k_{1}}}{\partial \ln C_{i k_{1}}} \frac{\partial Y_{j k_{2}}}{\partial \ln C_{j k_{2}}} \Delta \ln C_{i k_{1}} \Delta \ln C_{j k_{2}}
$$

where $Y_{i k}$ are the model expectation for the experimental rate $i$ due to the solar neutrino flux $k$ and $\Delta \ln C_{j k}$ are the fractional cross-sectional uncertainties (obtained from Reference [87]) with the values tabulated in Table 7.8. The delta function $\delta_{X(i), X(j)}$ accounts for the fact that only experiments with the same neutrino capture interactions $X(i)=X(j)$ are correlated due to the cross-section uncertainties. In particular, it gives a rise to off-diagonal elements in the crosssectional matrix only for the case of the two measurements from gallium experiments (Gallex/GNO and SAGE). 
Neutrino capture cross-section errors for the ES reaction at SK, SNO and Borexino are neglected in the analyses due to their smallness [84]. The errors on the neutrino-deuteron cross-sections through the $\mathrm{CC}$ and NC reactions at SNO are included in the systematic error matrix that has been presented in the previous section. They are $100 \%$ correlated between the different phases of SNO.

Equation (7.30) can be simplified as:

$$
\sigma_{i j}^{2}(\mathrm{cs})=\delta_{X(i), X(j)} \sum_{k_{1}=1}^{8} \sum_{k_{2}=1}^{8} \delta_{k_{1}} \delta_{k_{2}} \Delta Y_{i k_{1}} \Delta Y_{j k_{2}}
$$

with variations of the rates $\Delta Y_{j k}$ computed and re-scaled as $1 \sigma$ at each point in $\tan ^{2} \theta-\Delta m^{2}$ plane.

Table 7.8: The errors on the cross-section $\Delta \ln C_{i k}$ for the chlorine ( $1 \sigma$ values) and gallium ( $3 \sigma$ values) experiments. The numbers are obtained from Reference [87].

\begin{tabular}{ccccccccc}
\hline \hline experiment & pp & pep & hep & ${ }^{7} \mathrm{Be}$ & ${ }^{8} \mathrm{~B}$ & ${ }^{13} \mathrm{~N}$ & ${ }^{15} \mathrm{O}$ & ${ }^{17} \mathrm{~F}$ \\
\hline $\mathrm{Cl}$ & 0.0 & 0.02 & 0.037 & 0.02 & 0.032 & 0.020 & 0.020 & 0.020 \\
$\mathrm{Ga}$ & 0.023 & 0.17 & 0.32 & 0.07 & 0.32 & 0.06 & 0.12 & 0.12 \\
\hline \hline
\end{tabular}

The second contribution to the theoretical error matrix arises from the relevant astrophysical parameters that are used to construct the different solar models and which affect all the experiments (Homestake, Gallex/GNO, SAGE, SK, SNO and Borexino). The number of these parameters depends on the particular SSM used. The results presented here are obtained by using the solar models that are denoted as the SSM Bahcall-Pinsonneault-Basu 2000 (SSM BP00) [38], SSM Bahcall-Pinsonneault 2004 (SSM BP04) [39] and SSM Bahcall-Serenelli 2005 (SSM $\mathrm{BS} 05(\mathrm{OP}))[40]$.

The SSM BP00 and BP04 consider a set of eleven astrophysical parameters $X_{k}$ 
listed in Table 7.9: nuclear cross-section factors $\left(S_{11}, S_{33}, S_{34}, S_{1,14}\right.$ and $\left.S_{17}\right)$, the Sun luminosity $\left(\mathrm{L}_{\odot}\right)$, metallicity $(Z / X)$, the Sun's age, opacity ${ }^{2}$, element diffusion and cross-section $C_{\mathrm{Be}}$ for the ${ }^{7} \mathrm{Be}$ electron capture interaction ${ }^{7} \mathrm{Be}(\mathrm{e}, \mathrm{Li}) \nu_{\mathrm{e}}$. The latter affect only the ${ }^{8} \mathrm{~B}$ neutrino flux because ${ }^{7} \mathrm{Be}$ electron capture occurs much faster than ${ }^{7} \mathrm{Be}$ proton capture that produces ${ }^{8} \mathrm{~B}$ neutrinos. One of the the most important uncertainties in the SSM is the uncertainty on the cross-section factor $S_{34}$ for the interaction ${ }^{3} \mathrm{He}\left({ }^{4} \mathrm{He}, \gamma\right){ }^{7} \mathrm{Be}$. It affects the prediction on both the ${ }^{8} \mathrm{~B}$ and ${ }^{7}$ Be fluxes by $8 \%$. The SSM BP04 and BS05(OP) decompose the metallicity parameter $Z / X$ into additional nine individual contributions from the most important heavy elements in the solar composition: $\mathrm{C}, \mathrm{N}, \mathrm{O}, \mathrm{Ne}, \mathrm{Mg}, \mathrm{Si}, \mathrm{S}, \mathrm{Ar}$ and Fe [88]. The fractional uncertainties for $Z / X$ are listed in Table 7.10. BP04 and BS05(OP) also introduce one additional uncertainty $C_{h e p}$ on the cross-section in the reaction producing hep neutrinos.

Table 7.9: Fractional $1 \sigma$ uncertainties $\Delta \ln X_{k}$ associated with the astrophysical parameters $X_{k}$ taken from Bahcall's code exportrates. $f$ in Reference [43] for SSM $\mathrm{BP} 04$ and $\mathrm{BS} 05(\mathrm{OP})$.

\begin{tabular}{cccccccccc}
\hline \hline$S_{11}$ & $S_{33}$ & $S_{34}$ & $S_{1,14}$ & $\bar{S}_{17}$ & $L_{\odot}$ & $Z / X$ & $\bar{A} g e$ & $\bar{D}$ iff & $C_{\mathrm{Be}}$ \\
\hline 0.004 & 0.06 & 0.094 & 0.19 & 0.038 & 0.004 & 0.15 & 0.004 & 0.15 & 0.02 \\
\hline \hline
\end{tabular}

Table 7.10: Fractional $1 \sigma$ uncertainties $\Delta \ln X_{l k}$ for the decomposed metallicity parameters $Z_{l} / X$ taken from Bahcall's code exportrates. $f$ in Reference [43] for SSM $\mathrm{BP} 04$ and BS05(OP).

\begin{tabular}{cccccccccc}
\hline \hline$X_{l k}$ & $\overline{\mathrm{C}}$ & $\mathrm{N}$ & $\mathrm{O}$ & $\mathrm{Ne}$ & $\overline{\mathrm{Mg}}$ & $\mathrm{Si}$ & $\mathrm{S}$ & $\overline{\mathrm{Ar}}$ & $\mathrm{Fe}$ \\
\hline $1 \sigma$ & 0.297 & 0.320 & 0.387 & 0.539 & 0.115 & 0.115 & 0.092 & 0.496 & 0.115 \\
\hline \hline
\end{tabular}

\footnotetext{
${ }^{2}$ Opacity parameter uncertainties are provided in BP00, but not used.
} 
The variation of the solar neutrino fluxes $\phi_{i}$ induced by the change in the astrophysical parameters is quantified in terms of the logarithmic partial derivatives $\alpha_{i k}$ :

$$
\alpha_{i k}=\frac{\partial \ln \phi_{i}}{\partial \ln X_{k}}
$$

The logarithmic derivatives $\alpha_{i k}$ are listed in Tables 7.11 and 7.12. They are used to build the correlated astrophysical error matrix:

$$
\sigma_{i j}^{2}(\mathrm{ap})=\sum_{k=1}^{M} \frac{\partial Y_{i}}{\partial \ln X_{k}} \frac{\partial Y_{j}}{\partial \ln X_{k}}\left(\Delta \ln X_{k}\right)^{2}=\sum_{l_{1}=1}^{8} \sum_{l_{2}=1}^{8} Y_{i l_{1}} Y_{j l_{2}} \sum_{k=1}^{M} \alpha_{l_{1} k} \alpha_{l_{2} k}\left(\Delta \ln X_{k}\right)^{2} .
$$

It should be noted that the astrophysical error matrix must be used for solar neutrino fluxes that are fixed to the values predicted by the SSM. In the case when values for some of these fluxes are not fixed, but used as free parameters in the fit, the corresponding logarithmic derivatives must be set to zero. In the analyses presented in this chapter, only the ${ }^{8} \mathrm{~B}$ neutrino flux is used as a free parameter in the fit, hence the logarithmic derivatives $\alpha_{8_{\mathrm{B} k}}$ are set to zero.

The error matrix $\sigma_{i j}^{2}\left({ }^{8} \mathrm{~B}\right)$ for the uncertainty of the ${ }^{8} \mathrm{~B}$ spectrum shape is constructed by using partial derivatives $\beta_{i k}$ that are defined in Equation (7.18), where index $k={ }^{8} \mathrm{~B}$ will be omitted for simplicity in following equation:

$$
\sigma_{i j}^{2}\left({ }^{8} \mathrm{~B}\right)=\mathrm{Y}_{\mathrm{i}}^{\text {th }} \mathrm{Y}_{\mathrm{j}}^{\text {th }} \beta_{\mathrm{i}} \beta_{\mathrm{j}} \Delta \mathrm{S}_{\mathrm{i}} \Delta \mathrm{S}_{\mathrm{j}}=\frac{\partial \mathrm{Y}_{\mathrm{i}}^{\text {th }}}{\partial \mathrm{S}_{\mathrm{i}}} \frac{\partial \mathrm{Y}_{\mathrm{j}}^{\mathrm{th}}}{\partial \mathrm{S}_{\mathrm{j}}} \Delta \mathrm{S}_{\mathrm{i}} \Delta \mathrm{S}_{\mathrm{j}}
$$

with $\Delta S_{i}$ being the error on the ${ }^{8} \mathrm{~B}$ spectrum shape in bin $i$. This matrix is computed at each point in the $\tan ^{2} \theta-\Delta m^{2}$ plane and it correlates all solar neutrino experiments that are sensitive to the ${ }^{8} \mathrm{~B}$ neutrino flux. 
Table 7.11: Logarithmic partial derivatives on neutrino fluxes $\alpha_{i k}=\partial \ln \phi_{i} / \partial \ln X_{k}$ with respect to the nuclear cross-section factors, luminosity, metallicity, the Sun's age, element diffusion and ${ }^{7} \mathrm{Be}$ cross-section; taken from Bahcall's code exportrates.f in Reference [43] for SSM BP04 and BS05(OP). Fractional $3 \sigma$ uncertainties $\Delta Y_{i k}$ in respect to the opacity parameter are tabulated, while fractional $1 \sigma$ uncertainty $\Delta Y_{i k}$ in respect to the uncertainty $C_{h e p}$ applies only to the hep flux.

\begin{tabular}{lcccccccc}
\hline \hline$\alpha_{i k}$ & pp & pep & hep & ${ }^{7} \mathrm{Be}$ & ${ }^{8} \bar{B}$ & ${ }^{13} \mathrm{~N}$ & ${ }^{15} \mathrm{O}$ & ${ }^{17} \overline{\mathrm{F}}$ \\
\hline$S_{11}$ & 0.14 & -0.17 & -0.08 & -0.97 & -2.59 & -2.53 & -2.93 & -2.94 \\
$S_{33}$ & 0.03 & 0.05 & -0.45 & -0.43 & -0.40 & 0.02 & 0.02 & 0.02 \\
$S_{34}$ & -0.06 & -0.09 & -0.08 & 0.86 & 0.81 & -0.05 & -0.05 & -0.05 \\
$S_{1,14}$ & -0.02 & -0.02 & -0.01 & 0.0 & 0.01 & 0.85 & 1.0 & 0.01 \\
$S_{17}$ & 0.0 & 0.0 & 0.0 & 0.0 & 1.0 & 0.0 & 0.0 & 0.0 \\
$\mathrm{~L}_{\odot}$ & 0.73 & 0.87 & 0.12 & 3.4 & 6.76 & 5.16 & 5.94 & 6.25 \\
$Z / X$ & -0.08 & -0.17 & -0.22 & 0.58 & 1.265 & 1.86 & 2.03 & 2.09 \\
Age & -0.07 & 0.0 & -0.11 & 0.69 & 1.28 & 1.01 & 1.27 & 1.29 \\
Opa & 0.008 & 0.014 & 0.032 & -0.083 & -0.157 & -0.100 & -0.123 & -0.128 \\
Diff & 0.017 & 0.029 & 0.050 & -0.120 & -0.267 & -0.341 & -0.367 & -0.380 \\
$C_{\mathrm{Be}}$ & 0.0 & 0.0 & 0.0 & 0.0 & 1.0 & 0.0 & 0.0 & 0.0 \\
\hline$C_{\text {hep }}$ & 0.0 & 0.0 & 0.151 & 0.0 & 0.0 & 0.0 & 0.0 & 0.0 \\
\hline \hline
\end{tabular}

Table 7.12: Logarithmic partial derivatives on neutrino fluxes $\alpha_{i k}=\partial \ln \phi_{i} / \partial \ln X_{k}$ with respect to the individual metallicity uncertainties taken from Bahcall's code exportrates. $f$ in Reference [43] for the SSM BP04 and BS05(OP).

\begin{tabular}{lcccccccc}
\hline \hline$\alpha_{i k}$ & pp & pep & hep & ${ }^{7} \mathrm{Be}$ & ${ }^{8} \mathrm{~B}$ & ${ }^{13} \mathrm{~N}$ & ${ }^{15} \mathrm{O}$ & ${ }^{17} \mathrm{~F}$ \\
\hline $\mathrm{C}$ & -0.014 & -0.025 & -0.015 & -0.002 & 0.030 & 0.845 & 0.826 & 0.033 \\
$\mathrm{~N}$ & -0.003 & -0.006 & -0.004 & 0.002 & 0.011 & 0.181 & 0.209 & 0.010 \\
$\mathrm{O}$ & -0.006 & -0.011 & -0.023 & 0.052 & 0.121 & 0.079 & 0.093 & 1.102 \\
$\mathrm{Ne}$ & -0.005 & -0.005 & -0.017 & 0.049 & 0.096 & 0.057 & 0.068 & 0.076 \\
$\mathrm{Mg}$ & -0.005 & -0.005 & -0.018 & 0.051 & 0.096 & 0.060 & 0.070 & 0.078 \\
$\mathrm{Si}$ & -0.011 & -0.014 & -0.037 & 0.104 & 0.194 & 0.128 & 0.150 & 0.164 \\
$\mathrm{~S}$ & -0.008 & -0.017 & -0.028 & 0.074 & 0.137 & 0.094 & 0.109 & 0.120 \\
$\mathrm{Ar}$ & -0.002 & -0.006 & -0.007 & 0.018 & 0.034 & 0.024 & 0.028 & 0.031 \\
$\mathrm{Fe}$ & -0.023 & -0.065 & -0.069 & 0.209 & 0.515 & 0.342 & 0.401 & 0.444 \\
\hline \hline
\end{tabular}




\subsection{The Results}

In this section, the most recent results on solar neutrino oscillation analyses from the $\chi^{2}$ method, that appeared in the most recent SNO publication for SNOIII [51], are presented. The SNO-I and SNO-II results are shown as well with the newest model predictions from the SSM BS05(OP) and latest ${ }^{8} \mathrm{~B}$ spectrum from Reference [63]. In all these analyses, the ${ }^{8} \mathrm{~B}$ neutrino flux is a free parameter in the fit, while the hep neutrino flux is fixed to the predicted value from the SSM model.

The parameter ranges used in solar neutrino oscillation analyses are $\left[10^{-3}, 10\right]$ for $\tan ^{2} \theta$ and $\left[10^{-11}, 10^{-3}\right] \mathrm{eV}^{2}$ for $\Delta m^{2}$. The part of the $\tan ^{2} \theta-\Delta m^{2}$ plane in which both mixing parameters are large is known as the Large Mixing Angle (LMA) region. The part of the plane where the mixing angle is small is called the Small Mixing Angle (SMA) region. In the SMA region, the MSW effect can significantly enhance neutrino flavour conversion, even if the vacuum mixing is small. The other well-known part of the $\tan ^{2} \theta-\Delta m^{2}$ plane where the mixing can be large is known as the Low $\Delta m^{2}$ (LOW) region with the squared mass difference between $10^{-7}$ and $10^{-6} \mathrm{eV}^{2}$. The Quasi-Vacuum Oscillations (QVO) region is where the $\Delta m^{2}$ is between about $10^{-8}$ and $10^{-9} \mathrm{eV}^{2}$; while the Vacuum (VAC) region is for the lowest values of $\Delta m^{2}$ where the MSW effect is negligible since the propagation of neutrinos is similar to that in vacuum.

The $\chi^{2}$ function given in Equation (7.14), is minimized at each point in the $\tan ^{2} \theta-\Delta m^{2}$ plane with respect to the ${ }^{8} \mathrm{~B}$ neutrino flux. The least-square fit and the projection in the $\tan ^{2} \theta-\Delta m^{2}$ plane are then performed by allowing any values for the ${ }^{8} \mathrm{~B}$ neutrino flux for a given value for $\tan ^{2} \theta$ and $\Delta m^{2}$. At the minimum 
value of the $\chi_{\min }^{2}$, the best fit values for the mixing parameters $\tan ^{2} \theta$ and $\Delta m^{2}$ are extracted, together with the corresponding value for the ${ }^{8} \mathrm{~B}$ flux. Then, the $68 \%$, $95 \%$ and $99.78 \%$ confidence level (CL) regions in the two-dimensional parameter space $\tan ^{2} \theta-\Delta m^{2}$ are drawn, following the definitions:

$$
\begin{aligned}
& 68 \% \mathrm{CL}=\chi_{\min }^{2}+2.279, \\
& 95 \% \mathrm{CL}=\chi_{\min }^{2}+5.99, \\
& 99.73 \% \mathrm{CL}=\chi_{\min }^{2}+11.83 .
\end{aligned}
$$

The errors on the mixing parameters are determined by projecting the $\chi^{2}$ function at the best fit point on the $\tan ^{2} \theta$ and $\Delta m^{2}$ axes, separately. The onedimensional (1D) projections are not a simple slice of the two-dimensional contour at $\chi_{m i n}^{2}$, but instead a projection in which $\Delta \chi^{2}=\chi^{2}-\chi_{m i n}^{2}$ is computed for each 1D axis allowing the other 1D parameter to take any values. From these one dimensional projections the errors on each parameter are read as $1 \sigma$ standard spreads by looking at $\Delta \chi^{2}+1$ from $\chi_{\min }^{2}$.

\subsubsection{The Results - SNO-only}

The model predictions are recomputed for the neutrino yields for all solar neutrino experiments with the neutrino fluxes from the new SSM BS05(OP), which is in good agreement with the helioseismological data, and the latest ${ }^{8} \mathrm{~B}$ spectrum shape with its associated errors from Winter et al. [63]. The new model predictions are used for this analysis and the forthcoming SNO publications.

Before the phase III of the SNO experiment, the best fit values for the solar neutrino mixing parameters using the data from the SNO-I summed energy spectra 
from CC, ES and NC signals and backgrounds (for day and night), SNO-II NC and ES fluxes as well as CC kinetic energy energy spectra (for day and night), which are published in [48], are: $\Delta m^{2}=5.01_{-1.71}^{+6.20} \times 10^{-5} \mathrm{eV}^{2}$ and $\tan ^{2} \theta=0.445_{-0.097}^{+0.089}$. The flux of the ${ }^{8} \mathrm{~B}$ neutrinos is floated with respect to the SSM BS05(OP) prediction of $5.69 \times 10^{6} \mathrm{~cm}^{-2} \mathrm{~s}^{-1}$, and its best fitted value is $\phi_{8_{\mathrm{B}}}=5.15 \times 10^{6} \mathrm{~cm}^{-2} \mathrm{~s}^{-1}$. The flux of hep neutrinos is fixed at the SSM BP05(OP) value of $7.93 \times 10^{3} \mathrm{~cm}^{-2} \mathrm{~s}^{-1}$. The minimum $\chi^{2}$ at the best fit point is 68.97 for 69 degrees of freedom. The allowed regions at $68 \%, 95 \%$ and $99.73 \%$ confidence level (CL) in the $\Delta m^{2}-\tan ^{2} \theta$ plane from this fit are shown in Figure 7.2. The allowed regions correspond to the LMA, LOW, SMA and VAC parts of the oscillation plane. The best fit point lies in the LMA region.

Finally, with the inclusion of the latest data from the SNO NCD phase (SNOIII), the following neutrino mixing parameters are extracted: $\Delta m^{2}=4.57_{-1.22}^{+2.30} \times$ $10^{-5} \mathrm{eV}^{2}$ and $\tan ^{2} \theta=0.447_{-0.048}^{+0.045}$. The flux of the ${ }^{8} \mathrm{~B}$ neutrinos has been floated in respect to the SSM BS05(OP) prediction of $5.69 \times 10^{6} \mathrm{~cm}^{-2} \mathrm{~s}^{-1}$, and its best fitted value is $\phi_{\mathrm{B}_{\mathrm{B}}}=5.12 \times 10^{6} \mathrm{~cm}^{-2} \mathrm{~s}^{-1}$. The flux of hep neutrinos is fixed at the SSM BP05(OP) value of $7.93 \times 10^{3} \mathrm{~cm}^{-2} \mathrm{~s}^{-1}$. The minimum $\chi^{2}$ at the best fit point is 73.77 for 72 degrees of freedom. The allowed regions at $68 \%, 95 \%$ and 99.73\% confidence level (CL) in the $\Delta m^{2}-\tan ^{2} \theta$ plane from this fit (shown in Figure 7.3) are significantly improved compared to the previous results. The VAC region has been ruled out at the $99.73 \%$ CL for the first time using the SNO-only data. The remaining regions in the oscillation plane are significantly smaller than those shown in Figure 7.2, while marginalized $1 \sigma$ errors on the best fit values for the neutrino mixing parameters decreased by more than $40 \%$. The comparison of the two best fit results are given in Table 7.13 for before and after the inclusion 


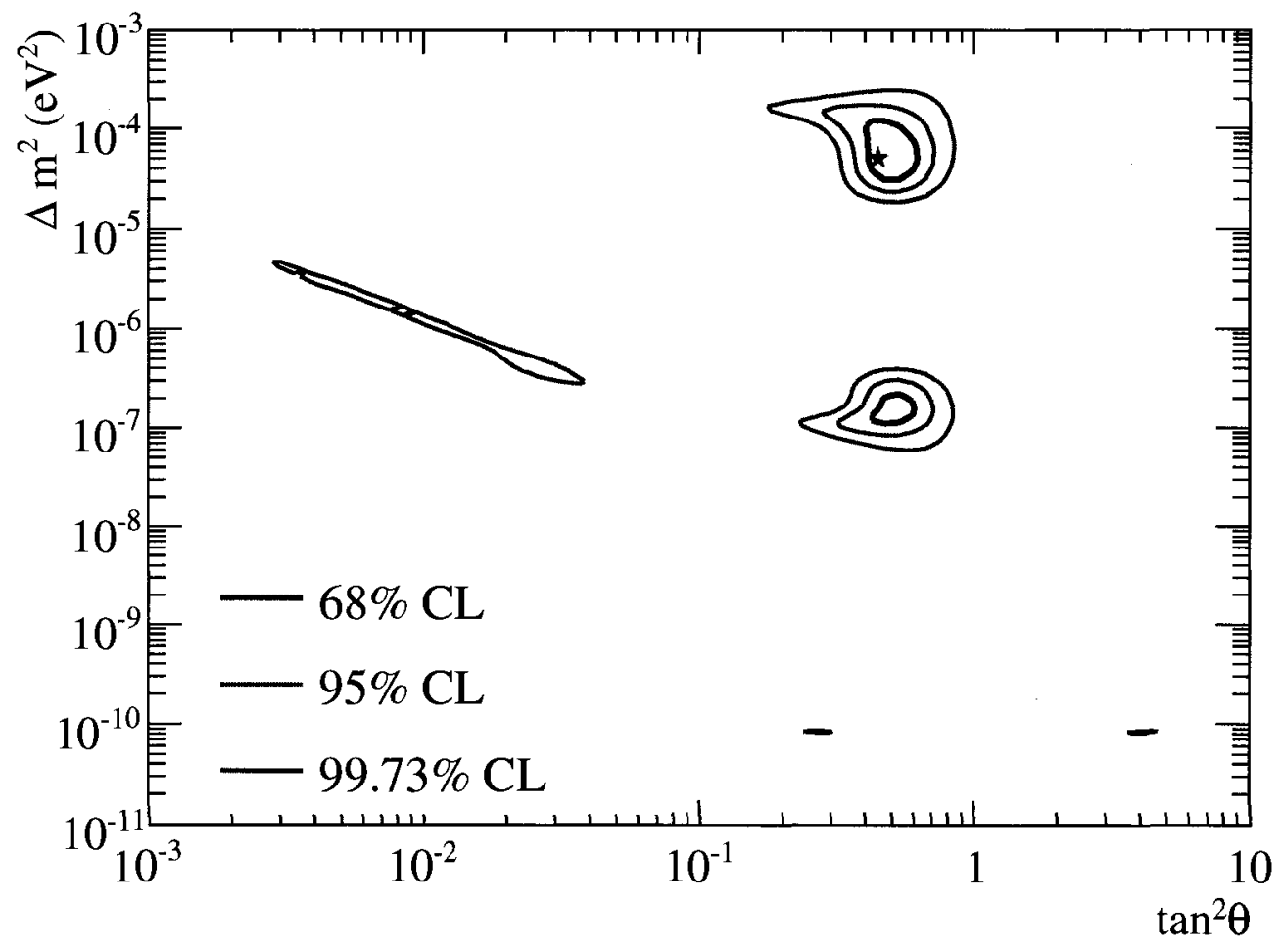

Figure 7.2: Before SNO-III: SNO-only neutrino oscillation confidence level contours for the combined SNO-I and SNO-II data samples using the model predictions from the SSM BS05(OP) and the ${ }^{8} \mathrm{~B}$ neutrino spectrum from Winter et al. This analysis includes the summed kinetic energy spectra from phase I (day and night); $\mathrm{NC}$ and ES fluxes, and CC kinetic energy spectra from phase II (day and night). The best fit point is at: $\Delta m^{2}=5.01 \times 10^{-5} \mathrm{eV}^{2}, \tan ^{2} \theta=0.447$, $\phi_{\mathrm{B}_{\mathrm{B}}}=5.15 \times 10^{6} \mathrm{~cm}^{-2} \mathrm{~s}^{-1}$. The hep neutrino flux was fixed at $7.93 \times 10^{3} \mathrm{~cm}^{-2} \mathrm{~s}^{-1}$. View this figure in colour. 
of the SNO-III data sample in the SNO-only oscillation analysis.

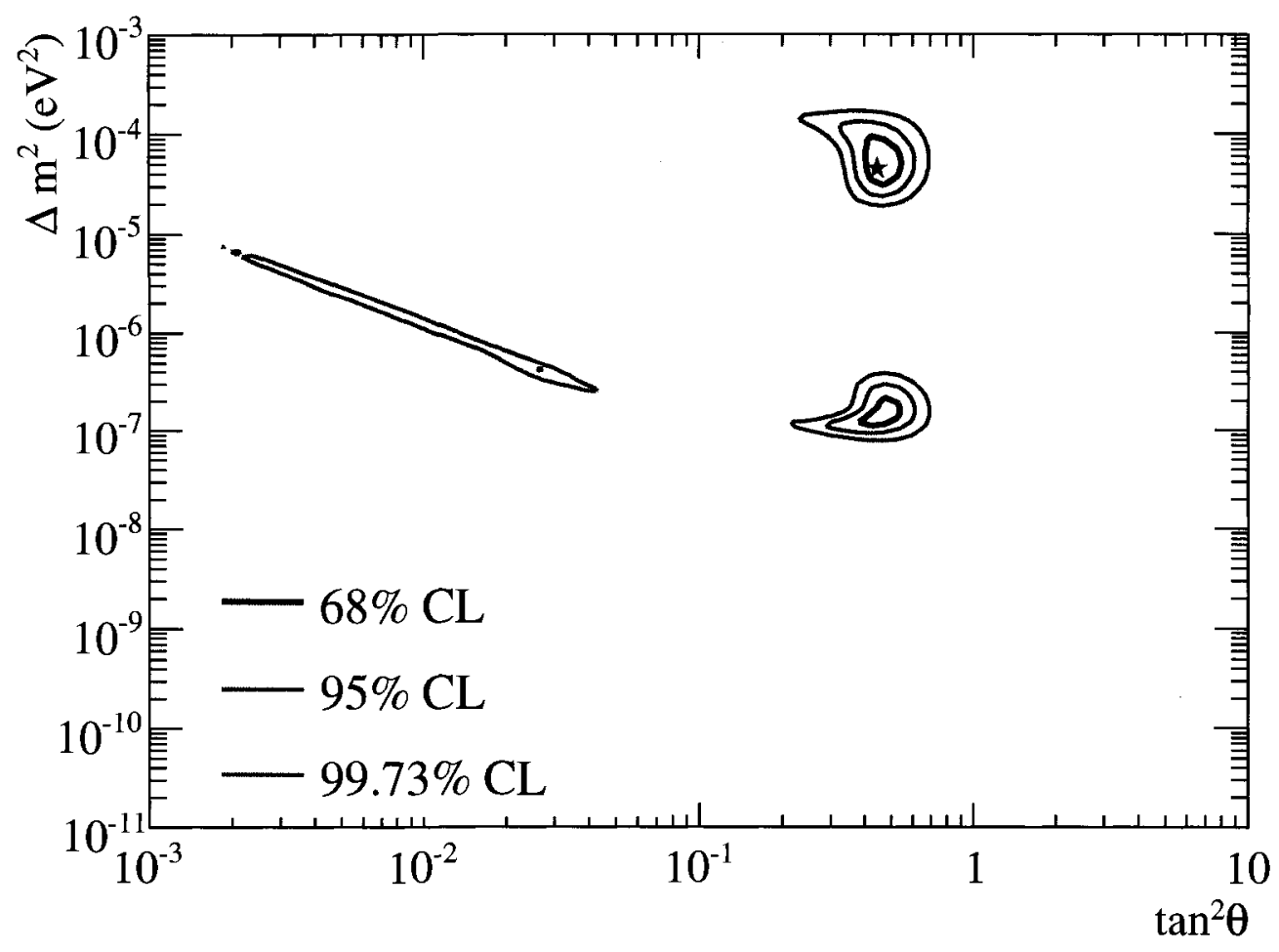

Figure 7.3: After SNO-III: SNO-only neutrino oscillation confidence level contours published in Reference [51]. This analysis includes the summed kinetic energy spectra from phase I (day and night); NC and ES fluxes, and CC kinetic energy spectra from phase II (day and night); and CC, NC and ES fluxes from phase III. The best fit point is at: $\Delta m^{2}=4.57 \times 10^{-5} \mathrm{eV}^{2}, \tan ^{2} \theta=0.447, \phi_{8_{\mathrm{B}}}=5.12 \times 10^{6}$ $\mathrm{cm}^{-2} \mathrm{~s}^{-1}$. The hep neutrino flux was fixed at $7.93 \times 10^{3} \mathrm{~cm}^{-2} \mathrm{~s}^{-1}$. View this figure in colour.

\subsubsection{Global Solar and Solar+KamLAND Results}

In this section the results from the global solar neutrino oscillation analyses before and after the latest NCD data from SNO are presented.

For the global solar analyses, that was published on February 25, 2005 in Ref- 
Table 7.13: SNO-only neutrino oscillation best-fit parameters.

\begin{tabular}{lccc}
\hline \hline Analysis & $\Delta m^{2}\left(10^{-5} \mathrm{eV}^{2}\right)$ & $\tan ^{2} \theta$ & ${ }^{8} \mathrm{~B}$ flux $\left(10^{6} \mathrm{~cm}^{-2} \mathrm{~s}^{-1}\right)$ \\
\hline Before SNO-III & $5.01_{-1.71}^{+6.20}$ & $0.45_{-0.10}^{+0.09}$ & 5.15 \\
After SNO-III & $4.57_{-1.22}^{+2.30}$ & $0.45_{-0.05}^{+0.05}$ & 5.12 \\
\hline \hline
\end{tabular}

erence [48], the following data were used: the rate measurements from Homestake, Gallex/GNO and SAGE; SK-I zenith energy spectra from Reference [13]; summed kinetic energy spectra from the SNO-I phase (day and night); NC and ES fluxes, and CC kinetic energy spectra from SNO-II (day and night). The model predictions are obtained by using the standard solar models BP00 and BP04, in the same manner as it is described in the previous section. The left panel in Figure 7.4 shows the allowed region in the solar neutrino mixing parameter space. The best fit parameters using these data are: $\Delta m^{2}=6.5_{-2.3}^{+4.4} \times 10^{-5} \mathrm{eV}^{2}$ and $\theta=33.9_{-2.5}^{+2.4}$, the ${ }^{8} \mathrm{~B}$ flux of $\phi_{8_{\mathrm{B}}}=5.06 \times 10^{6} \mathrm{~cm}^{-2} \mathrm{~s}^{-1}$. The minimum $\chi^{2}$ at the best fit point is 113.1 for 116 degrees of freedom. These global solar neutrino $\chi^{2}$ results were combined with the 766 ton-year data from KamLAND [89] reactor antineutrino experiment, which has better sensitivity to $\Delta m^{2}$ parameter than solar neutrino experiments. In general, electron antineutrino data from reactors can be combined with the solar neutrino data in case that the CPT invariance is preserved. This means that probability of electron neutrino flavour changing into the active neutrino states $P_{e a}$ is equal to that of active antineutrino changing into the electron antineutrino $P_{a e}$. The neutrino mixing parameters extracted from the Solar+KamLAND analysis are: $\Delta m^{2}=8.0_{-0.4}^{+0.6} \times 10^{-5} \mathrm{eV}^{2}$ and $\theta=33.9_{-2.2}^{+2.4}$, with the ${ }^{8} \mathrm{~B}$ flux of $\phi_{8_{\mathrm{B}}}=4.93 \times 10^{6} \mathrm{~cm}^{-2} \mathrm{~s}^{-1}$. The first impact of KamLAND is seen 

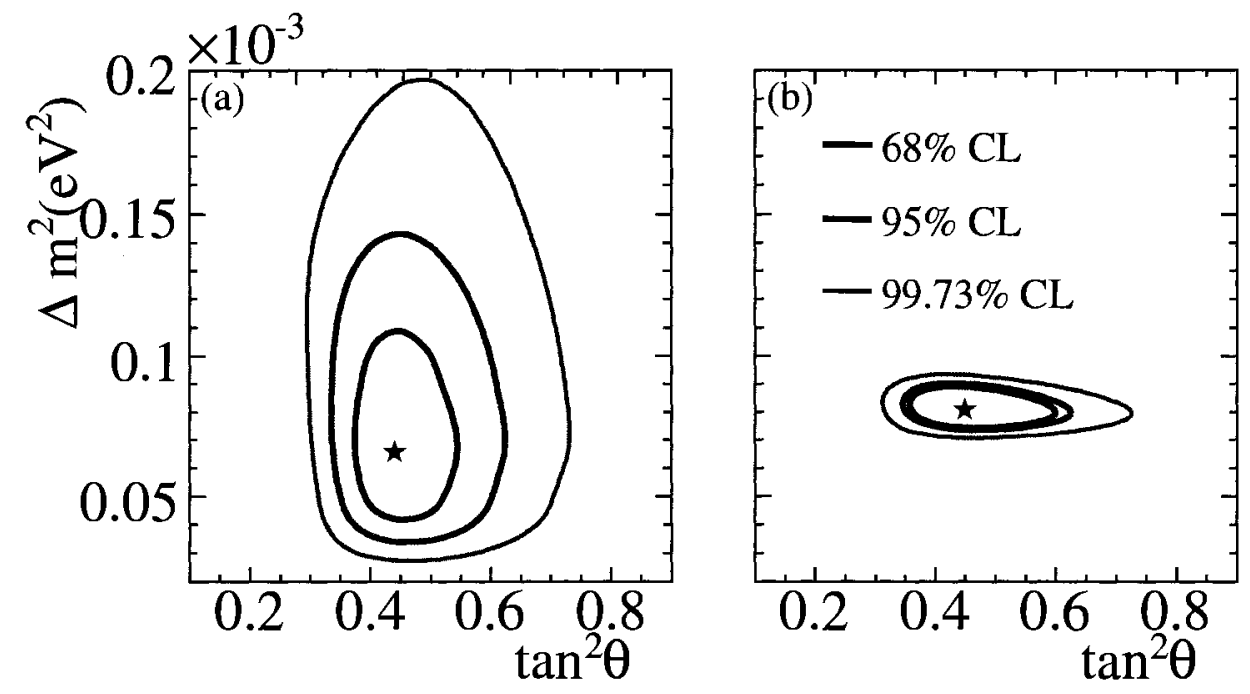

Figure 7.4: Before SNO-III: Neutrino oscillation confidence level contours published in Reference [48]. (a) Global solar analysis before Borexino including the rate measurements from Homestake, Gallex/GNO and SAGE, old SK-I zenithenergy spectra from [13]; summed kinetic energy spectra from SNO-I (day and night); NC and ES fluxes, and CC kinetic energy spectra from SNO-II (day and night). The best fit point is at: $\Delta m^{2}=6.5 \times 10^{-5} \mathrm{eV}^{2}, \tan ^{2} \theta=0.45$, $\phi_{8_{\mathrm{B}}}=5.06 \times 10^{6} \mathrm{~cm}^{-2} \mathrm{~s}^{-1}$. The hep is fixed at $9.3 \times 10^{3} \mathrm{~cm}^{-2} \mathrm{~s}^{-1}$; (b) Including the old KamLAND data from [89]. The best fit point is at: $\Delta m^{2}=8.0 \times 10^{-5}$ $\mathrm{eV}^{2}, \tan ^{2} \theta=0.437, \phi_{8_{\mathrm{B}}}=4.93 \times 10^{6} \mathrm{~cm}^{-2} \mathrm{~s}^{-1}$. View this figure in colour. 
in a much better precision obtained for the fitted $\Delta m^{2}$, in comparison to its value from the solar analysis only, as it can be seen in the right panel in Figure 7.4. The second impact is shown as a shift of $\Delta m^{2}$ parameter to the higher value, but yet consistent within $\pm 1 \sigma$ uncertainties of the global solar fit. The uncertainties on the neutrino mixing parameters are determined from one dimensional projections of the $\Delta \chi^{2}$ function, that are shown in Figure 7.5.

For the newest publication of SNO [51], the following changes in the global solar neutrino analysis are made: the model predictions for all solar neutrino experiments are computed using the SSM BS05(OP) and ${ }^{8} \mathrm{~B}$ neutrino spectrum shape from Reference [63], the newest result from Borexino is included [47], data from SK-1 are updated using results from Reference [72], and, most importantly, the new measurements from the SNO-III phase are incorporated. The global fit to these data leads to the following neutrino mixing parameters: $\Delta m^{2}=4.90_{-0.93}^{+1.64} \times$ $10^{-5} \mathrm{eV}^{2}$ and $\theta=33.5_{-1.3}^{+1.3}$, the ${ }^{8} \mathrm{~B}$ flux of $\phi_{8_{\mathrm{B}}}=5.21 \times 10^{6} \mathrm{~cm}^{-2} \mathrm{~s}^{-1}$. The minimum $\chi^{2}$ at the best fit point is 130.29 for 120 degrees of freedom. The allowed regions from this analysis are shown on the left panel in Figure 7.6. The constraint on both neutrino mixing parameters is much better than the 2005 result depicted in Figure 7.4. This result is combined with the latest results from KamLAND [90], leading to the following best fit parameters: $\Delta m^{2}=7.59_{-0.19}^{+0.21} \times 10^{-5} \mathrm{eV}^{2}$ and $\theta=34.4_{-1.2}^{+1.3}$, the ${ }^{8} \mathrm{~B}$ flux of $\phi_{8_{\mathrm{B}}}=4.92 \times 10^{6} \mathrm{~cm}^{-2} \mathrm{~s}^{-1}$. The great improvement in comparison with the former analysis is observed in the allowed regions from the combined fit shown on the right panel in Figure 7.6. The comparison between the results from global solar and global solar plus KamLAND analyses before and after SNO-III phase data are given in Table 7.14. The one dimensional projections of $\Delta \chi^{2}$ function, from which the errors on the mixing parameters are read, are 

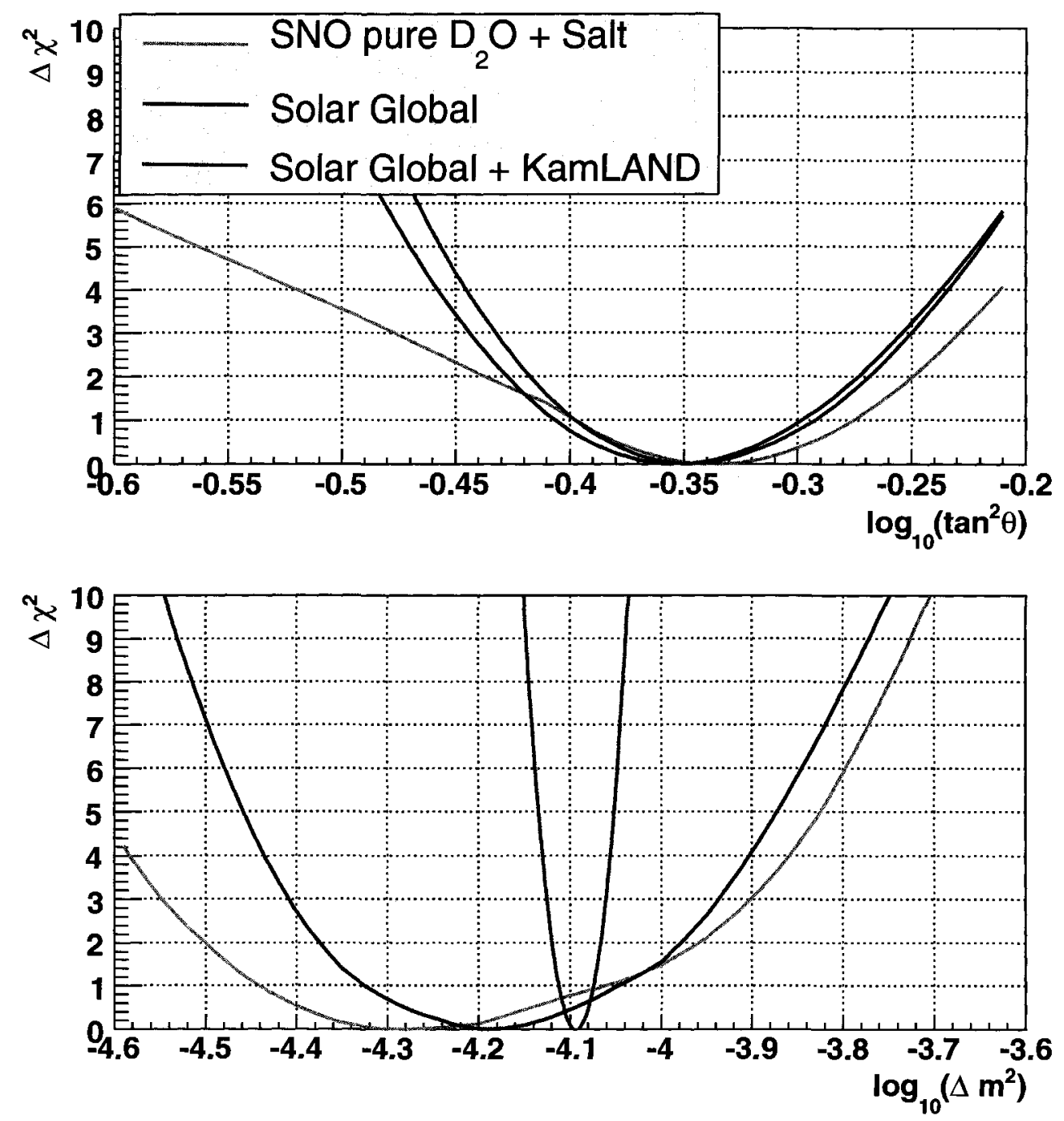

Figure 7.5: One dimensional projections of the $\Delta \chi^{2}$ function on $\tan ^{2} \theta$ and $\Delta m^{2}$ axis for SNO-only, global solar and global solar+KamLAND analyses before results from the SNO-III phase. View this figure in colour. 
depicted in Figure 7.7.
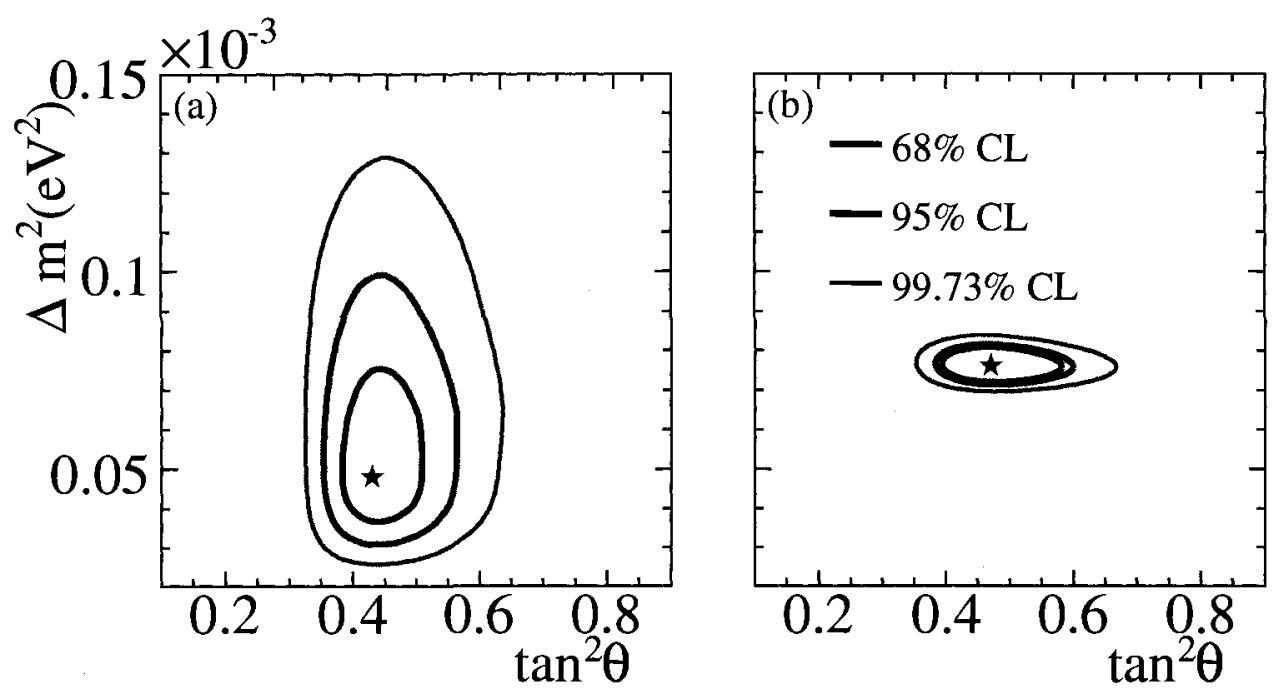

Figure 7.6: After SNO-III: Neutrino oscillation confidence level contours published in Reference [51]. (a) Global solar analysis including the rate measurements from Homestake, Gallex/GNO, SAGE and Borexino; newest SK-I zenith-energy spectra from [72], summed kinetic energy spectra from SNO-I (day and night); NC and ES fluxes, and CC kinetic energy spectra from SNO-II (day and night); and CC, ES and NC fluxes from SNO-III. The best fit point is at: $\Delta m^{2}=4.90 \times 10^{-5}$ $\mathrm{eV}^{2}, \tan ^{2} \theta=0.437, \phi_{8_{\mathrm{B}}}=5.21 \times 10^{6} \mathrm{~cm}^{-2} \mathrm{~s}^{-1}$. The hep neutrino flux is fixed at $7.93 \times 10^{3} \mathrm{~cm}^{-2} \mathrm{~s}^{-1}$; (b) Including the newest KamLAND data from [90]. The best fit point is at: $\Delta m^{2}=7.59 \times 10^{-5} \mathrm{eV}^{2}, \tan ^{2} \theta=0.468, \phi_{8_{\mathrm{B}}}=4.92 \times 10^{6} \mathrm{~cm}^{-2} \mathrm{~s}^{-1}$. View this figure in colour.

\subsection{Summary}

The general $\chi^{2}$ method used for the extraction of the neutrino mixing parameters from the solar neutrino data has been presented. The latest results from global solar neutrino oscillation analysis provide significant improvements on the allowed regions in the $\tan ^{2} \theta-\Delta m^{2}$ parameter plane and further confirm the predictions on neutrino flavour change from the two-neutrino oscillation hypothesis with MSW 

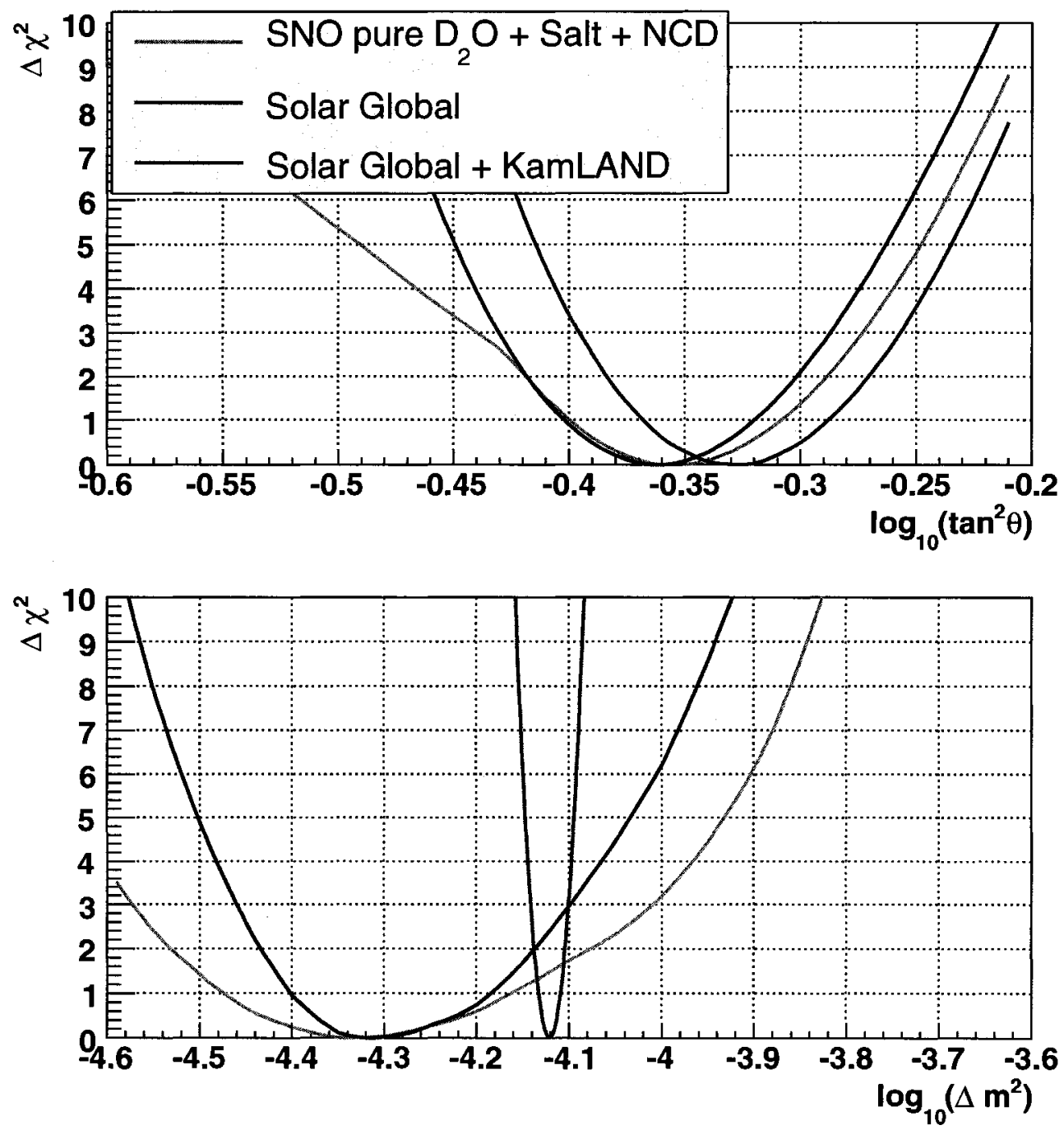

Figure 7.7: One dimensional projections of the $\Delta \chi^{2}$ function on $\tan ^{2} \theta$ and $\Delta m^{2}$ axis for SNO-only, global solar and global solar+KamLAND analyses after results from the SNO-III phase. View this figure in colour. 
Table 7.14: Global solar only and global solar+KamLAND best-fit parameters. The global solar results before the SNO-III phase do not include data from Borexino. The global solar+KamLAND results after SNO-III include the latest data from Borexino [47] and KamLAND [90].

\begin{tabular}{lcrc}
\hline \hline Analysis & $\Delta m^{2}\left(10^{-5} \mathrm{eV}^{2}\right)$ & $\tan ^{2} \theta$ & ${ }^{8} \mathrm{~B}$ flux $\left(10^{6} \mathrm{~cm}^{-2} \mathrm{~S}^{-1}\right)$ \\
\hline \multicolumn{4}{c}{ Before SNO-III phase } \\
\hline Global solar & $6.5_{-2.3}^{+4.4}$ & $0.45_{-0.08}^{+0.09}$ & 5.06 \\
with KamLAND & $8.0_{-0.4}^{+0.6}$ & $0.45_{-0.07}^{+0.09}$ & 4.93 \\
\hline \multicolumn{4}{c}{ After SNO-III phase } \\
\hline Global solar & $4.90_{-0.93}^{+1.64}$ & $0.44_{-0.04}^{+0.05}$ & 5.21 \\
with KamLAND & $7.59_{-0.19}^{+0.21}$ & $0.47_{-0.04}^{+0.05}$ & 4.92 \\
\hline \hline
\end{tabular}

effect. In comparison to older results, the new combined fit with the solar neutrino data and the latest results from KamLAND improved the constraints on the neutrino mixing parameters: mixing angle $\theta$ and $\Delta m^{2}$ by $45 \%$ and $60 \%$, respectively. The improvement on $\theta \equiv \theta_{12}$ is dominated by the SNO experiment and the new NCD results. The fitted values for the ${ }^{8} \mathrm{~B}$ neutrino flux are in agreement with the recent predictions from the SSM. 


\section{Chapter 8}

\section{Results - the Sterile Neutrino}

\section{Model}

An interpretation of the solar neutrino data, under the assumption of a weakly mixed sterile neutrino model as described in Chapter 2 (Sections 2.4 .2 and 2.5.2), is presented in this chapter. The model is applied to data samples from the three phases of the SNO experiment, as well as to the solar neutrino measurements from other experiments that were previously described in this thesis. The constraint on the allowed region in the $\sin ^{2} 2 \alpha-R_{\Delta}$ plane is placed for the first time; and the best fit values for sterile neutrino mixing parameters are extracted for a fixed value of $\Delta m^{2} \equiv \Delta m_{12}^{2}$ and $\tan ^{2} \theta \equiv \tan ^{2} \theta_{12}$.

\subsection{Motivation}

The latest results on the solar neutrino mixing parameters $\Delta m^{2}$ and $\tan ^{2} \theta$ (as shown in Table 7.14) from the combined analyses of the global solar neutrino data 
and the KamLAND reactor antineutrino measurement, were extracted by using the predictions from the two-neutrino mixing model. This result confirmed the results from previous publications of SNO $[48,73]$ that the Large Mixing Angle (LMA) region is a preferred region in solar neutrino mixing parameter space, as depicted in Figure 7.6.

However, a few predictions from the LMA solution have not been confirmed yet. There is still a possibility for some rare effects that could be explained with physics beyond the standard active neutrino mixing model and the LMA scenario. The LMA solution predicts that the survival probability $P_{e e}$ for ${ }^{8} \mathrm{~B}$ electron neutrino increases with the decrease of neutrino energy, as shown in Figure 8.1. As a consequence of this, it is expected to observe the upturn in the electron energy spectra bellow, approximately, $8 \mathrm{MeV}$, at the SNO and Super-Kamiokande (SK) experiments. These upturns have not been observed neither at SNO or SK, yet. Figure 8.2 shows the observed CC kinetic energy spectra from the SNO-II phase with the LMA expectation derived from the solar global + KamLAND best fit parameters extracted before the SNO-III phase $\left(\Delta m^{2}=8.0_{-0.4}^{+0.6} \times 10^{-5} \mathrm{eV}^{2}\right.$ and $\tan ^{2} \theta=0.45_{-0.07}^{+0.09}$ from Table 7.14).

Another plausibility for new effects beyond the SSM rises from the rate measurement at the Homestake experiment $R_{\mathrm{Cl}}=2.56_{-0.23}^{+0.23} \mathrm{SNU}$, that disagrees with the LMA expectation of 3.03 SNU at about the $2 \sigma$ level.

When applied to solar neutrinos, the model with two active and a single weakly mixed sterile neutrino could explain both the non-observation of the upturn in the neutrino energy spectra at SNO and SK, and also the lower rate observed by the Homestake experiment.

The assumptions from the model provide that the active neutrino states $\left(\nu_{e}\right.$ 


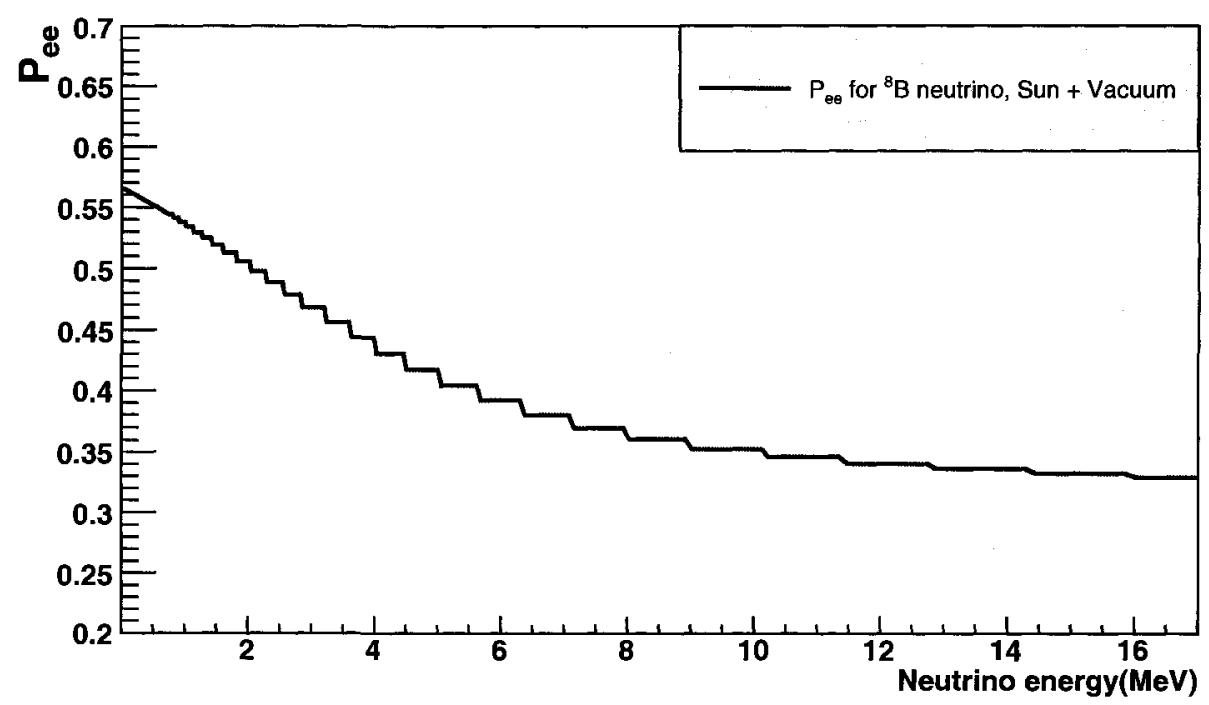

Figure 8.1: ${ }^{8} \mathrm{~B}$ neutrino survival probability $P_{e e}$ after propagation inside the Sun and vacuum for $\Delta m_{21}^{2}=7.6 \times 10^{-5} \mathrm{eV}^{2}$ and $\tan ^{2} \theta=0.47$.

and $\nu_{a}$ ) are described by the mixing parameters $\Delta m^{2} \equiv \Delta m_{12}^{2}$ and $\theta \equiv \theta_{12}$, while the sterile component is governed by $\sin ^{2} 2 \alpha$ and $R_{\Delta}$. For the reasons that have been already explained in Section 2.4.2, the sterile neutrino mixing parameters are assumed to be within the range:

$$
\begin{aligned}
R_{\Delta} & =[0,0.25], \\
\sin ^{2} 2 \alpha & =\left[10^{-5}, 10^{-2}\right] .
\end{aligned}
$$

The parameter space used in our analyses, contains 51 evenly spaced bins in $R_{\Delta} \in[0.0,0.25]$ and 61 evenly spaced bins in $\log \left(\sin ^{2} 2 \alpha\right) \in[-2,-5]$. Both the solar model and the LMA parameters $\left(\Delta m_{21}^{2}=7.1 \times 10^{-5} \mathrm{eV}^{2}\right.$ and $\left.\tan ^{2} \theta=0.40\right)$ are identical to those used in Reference [30]. 


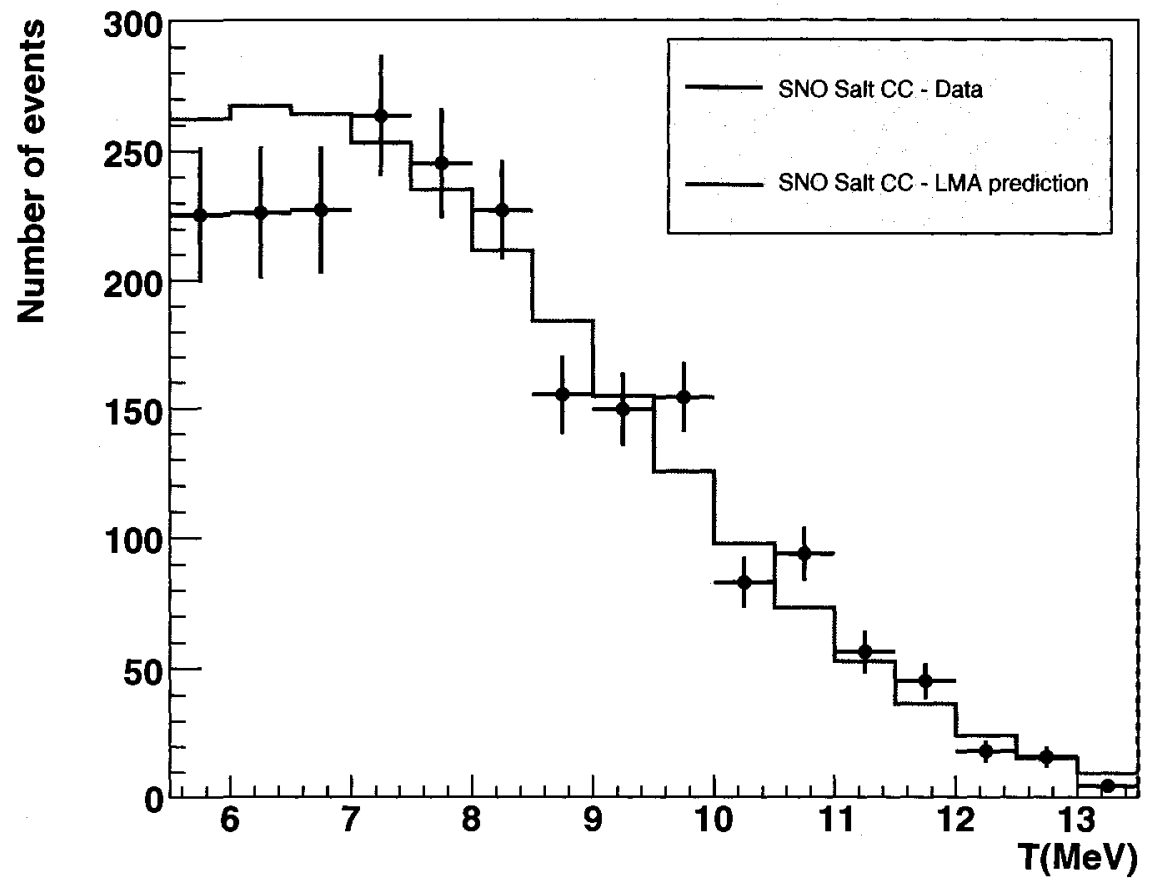

Figure 8.2: SNO-II CC kinetic energy spectrum compared with the LMA prediction for the global + KamLAND best fit point before SNO-III: $\Delta m_{21}^{2}=8.0 \times 10^{-5}$ $\mathrm{eV}^{2}, \tan ^{2} \theta=0.45, \phi_{8_{\mathrm{B}}}=4.93 \times 10^{6} \mathrm{~cm}^{-2} \mathrm{~s}^{-1}$ and $\phi_{h e p}=9.3 \times 10^{3} \mathrm{~cm}^{-2} \mathrm{~s}^{-1}$. 


\subsection{Solar Neutrino Transition Probabilities}

The impact of the weakly mixed sterile neutrino on the solar neutrino transition and survival probabilities is described in this section.

In general, each neutrino state $\nu_{\alpha}(\alpha=e, a, s)$ within this model is described with real and imaginary parts of a neutrino wave function:

$$
\left|\nu_{\alpha}\right\rangle=\operatorname{Re}\left(\left|\nu_{\alpha}\right\rangle\right)+i \operatorname{Im}\left(\left|\nu_{\alpha}\right\rangle\right) .
$$

Hence the propagation equation shown in Equation (2.53) represents a system of six coupled differential equations in flavour eigenstate basis. Knowing the initial conditions for the neutrino amplitudes $\operatorname{Re}\left(\left|\nu_{\alpha}(0)\right\rangle\right)$ and $\operatorname{Im}\left(\left|\nu_{\alpha}(0)\right\rangle\right)$ for the production point $x=0$ (or equivalently at time $t=0$ ), one has to solve the propagation equation for the neutrino amplitudes $\operatorname{Re}\left(\left|\nu_{\alpha}(L)\right\rangle\right)$ and $\operatorname{Im}\left(\left|\nu_{\alpha}(L)\right\rangle\right)$ at the detection point $x=L$. The transition probabilities $P_{\nu_{\alpha} \rightarrow \nu_{\beta}}$ are given then by:

$$
P_{\nu_{\alpha} \rightarrow \nu_{\beta}}=\mid\left.\operatorname{Re}\left(\left|\nu_{\beta}(L)\right\rangle\right)\right|^{2}+\mid\left.\operatorname{Im}\left(\left|\nu_{\beta}(L)\right\rangle\right)\right|^{2} .
$$

\subsubsection{Neutrino Propagation for the Sun and Vacuum}

For solar neutrinos, the propagation equation from their production region to the solar surface is solved numerically with the Hamiltonian from Equation (2.56). The neutrino production region in the Sun is sampled in 41000 equidistant radial points in the region of $R / R_{s u n} \in[0.0,0.41]$ for each of 11 planes in a single hemisphere that is parallel to the solar equator. This choice of production region is very similar to that used in References $[30,91]$. At the solar surface $R=R_{\odot}$, 
an instantaneous value for the wave function describing the final neutrino state is obtained:

$$
\left|\nu\left(R_{\odot}\right)\right\rangle=c_{s}\left|\nu_{s}\right\rangle+c_{e}\left|\nu_{e}\right\rangle+c_{a}\left|\nu_{a}\right\rangle
$$

where $c_{\alpha}(\alpha=s, e, a)$ are the transition amplitudes.

Due to the extreme conditions in the solar core, nuclei that produce neutrinos move and collide rapidly, hence they produce wave packets with a short coherence length equal to $10^{-8} \mathrm{~m}$ [31]. For a detailed treatment of the neutrino production processes see, for example, Reference [27]. Therefore, neutrino mass eigenstates reach the solar surface as incoherent beams, which allows for phase averaging over the final neutrino states that reach the vacuum. The phase averaging at that point also accounts for the neutrino propagation in the vacuum. In order to perform the averaging, the flavour eigenstate amplitudes $c_{\alpha}$ are expressed in terms of mass eigenstate amplitudes in vacuum $c_{i}(i=0,1,2)$ :

$$
\begin{aligned}
& c_{s}(t)=U_{00} c_{0}(t)+U_{01} c_{1}(t)+U_{02} c_{2}(t), \\
& c_{e}(t)=U_{10} c_{0}(t)+U_{11} c_{1}(t)+U_{12} c_{2}(t), \\
& c_{a}(t)=U_{20} c_{0}(t)+U_{21} c_{1}(t)+U_{22} c_{2}(t),
\end{aligned}
$$

where $U_{i j}$ are the elements of the mixing matrix given in Equation (2.37). At the time $t$, the mass eigenstates $c_{i}$ acquire the phases:

$$
c_{i}(t)=c_{i} \mathrm{e}^{-i \Phi_{i}}
$$

where after a re-phasing only two independent phases remain. From Equation (8.3), the survival $P_{e e}^{\odot}$ and transition $P_{e \beta}^{\odot}(\beta=s, a)$ probabilities for an electron 
neutrino that is originally produced in the Sun are derived as:

$$
\begin{aligned}
& P_{e e}^{\odot}=\left|c_{e}(t)\right|^{2}, \\
& P_{e s}^{\odot}=\left|c_{s}(t)\right|^{2}, \\
& P_{e a}^{\odot}=1-\left(P_{e e}^{\odot}+P_{e s}^{\odot}\right) .
\end{aligned}
$$

With the phase averaging, all the cross terms in the squared amplitudes $\left|c_{\alpha}(t)\right|^{2}$ cancel. Hence the final averaged probabilities for the Sun and vacuum have the form:

$$
\begin{aligned}
& \bar{P}_{e e}^{\odot}=U_{10}^{2}\left|c_{0}\right|^{2}+U_{11}^{2}\left|c_{1}\right|^{2}+U_{12}^{2}\left|c_{2}\right|^{2}, \\
& \bar{P}_{e s}^{\odot}=U_{00}^{2}\left|c_{0}\right|^{2}+U_{01}^{2}\left|c_{1}\right|^{2}+U_{02}^{2}\left|c_{2}\right|^{2}, \\
& \bar{P}_{e a}^{\odot}=1-\left(\bar{P}_{e e}^{\odot}+\bar{P}_{e s}^{\odot}\right) .
\end{aligned}
$$

These survival probabilities are further averaged over the production regions for the eight different neutrino sources inside the solar core $\left(p p, p e p\right.$, hep $,{ }^{8} \mathrm{~B},{ }^{7} \mathrm{Be}$, ${ }^{13} \mathrm{~N},{ }^{15} \mathrm{O}$ and ${ }^{17} \mathrm{~F}$ ) that are depicted in Figure 7.1.

The mixing with the sterile neutrino affects the solar neutrino survival probabilities $\bar{P}_{e e}^{\odot}$ by an appearance of a dip in the low energy range and flattening of the survival probabilities at intermediate energy $\left(E_{\nu} \sim 10 \mathrm{MeV}\right)$. It can be seen in Figure 8.3 that the size of the dip increases with larger sterile mixing angle $\alpha$, while the position of the dip shifts towards the intermediate energy range with larger ratio $R_{\Delta}$. A projection of the survival probabilities for $5 \mathrm{MeV}^{8} \mathrm{~B}$ neutrino in the $\sin ^{2} 2 \alpha-R_{\Delta}$ parameter space is shown in Figure 8.4. The appearance of this dip in $\bar{P}_{e e}^{\odot}$ could explain the lower rate at the Homestake detector, while the flattening of the $\bar{P}_{e e}^{\odot}$ could be a possible explanation for a non-observation of the upturn in the energy spectra at the SNO and SK experiments. 

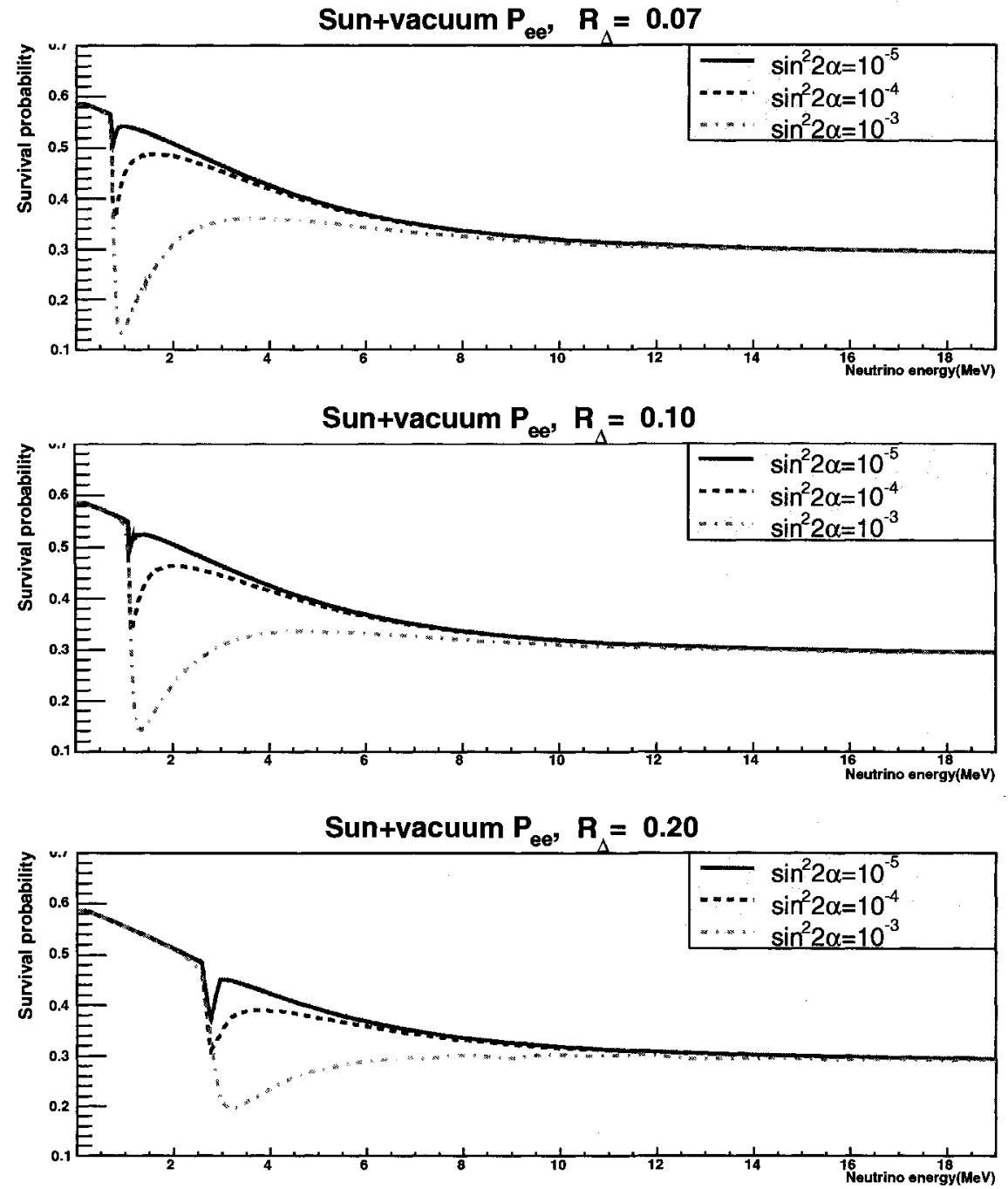

Figure 8.3: Survival probability $\bar{P}_{e e}^{\odot}$ for ${ }^{8} \mathrm{~B}$ electron neutrino and sterile mixing angles of $\sin ^{2} 2 \alpha=10^{-5}$ (solid), $\sin ^{2} 2 \alpha=10^{-4}$ (dotted) and $\sin ^{2} 2 \alpha=10^{-3}$ (dashed) for each of the three ratios $R_{\Delta}=0.07$ (upper graph), $R_{\Delta}=0.10$ (middle graph) and $R_{\Delta}=0.20$ (bottom graph). The LMA parameters used are $\Delta m_{21}^{2}=$ $7.1 \times 10^{-5} \mathrm{eV}^{2}$ and $\tan ^{2} \theta=0.40$. 


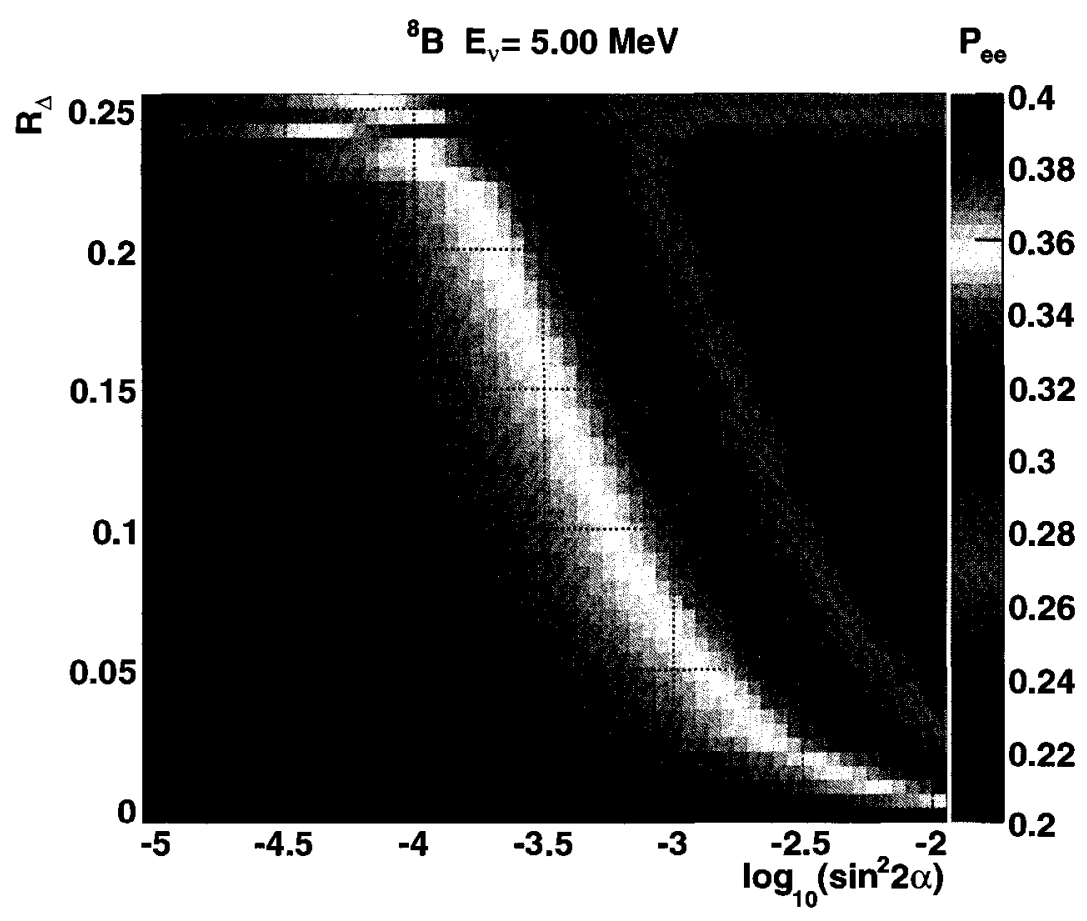

Figure 8.4: Survival probability $\bar{P}_{e e}^{\odot}$ for ${ }^{8} \mathrm{~B}$ electron neutrino with $E_{\nu}=5 \mathrm{MeV}$ projected in the $\sin ^{2} 2 \alpha-R_{\Delta}$ parameter space. The LMA parameters used are $\Delta m_{21}^{2}=7.1 \times 10^{-5} \mathrm{eV}^{2}$ and $\tan ^{2} \theta=0.40$. View this figure in colour. 


\subsubsection{The Earth Effect}

Due to the loss of coherence, solar neutrinos emerge from the Sun as incoherent beams of mass eigenstates. These neutrinos traverse the Earth along many trajectories, therefore one must solve the neutrino propagation Equation (2.53) for various neutrino paths inside the Earth. Instead of solving all these equations for many different initial conditions, which depend not only on the sterile neutrino mixing parametes, but also on the neutrino production point, the general method proposed in Reference [92] and further developed for the three-active neutrino case in Reference [93] was modified and applied to this model. The method decouples the propagation of neutrinos inside the Sun and through the vacuum from the propagation inside the Earth. The propagation equation for neutrino system in the Earth is solved for three different initial conditions (pure $\nu_{s}, \nu_{e}$ and $\nu_{a}$ states) at each point in the sterile neutrino mixing parameter space:

$$
\begin{aligned}
& \varphi_{s}(0)=\left|\nu_{s}\right\rangle, \\
& \varphi_{e}(0)=\left|\nu_{e}\right\rangle, \\
& \varphi_{a}(0)=\left|\nu_{a}\right\rangle .
\end{aligned}
$$

After passing some trajectory $r$ inside the Earth, these pure flavour states become linear combinations of all three neutrino states:

$$
\begin{aligned}
& \varphi_{s}(r)=a_{s}^{s}\left|\nu_{s}\right\rangle+a_{e}^{s}\left|\nu_{e}\right\rangle+a_{a}^{s}\left|\nu_{a}\right\rangle, \\
& \varphi_{e}(r)=a_{s}^{e}\left|\nu_{s}\right\rangle+a_{e}^{e}\left|\nu_{e}\right\rangle+a_{a}^{e}\left|\nu_{a}\right\rangle, \\
& \varphi_{a}(r)=a_{s}^{a}\left|\nu_{s}\right\rangle+a_{e}^{a}\left|\nu_{e}\right\rangle+a_{a}^{a}\left|\nu_{a}\right\rangle .
\end{aligned}
$$


The neutrino amplitudes for the pure neutrino states propagating inside the

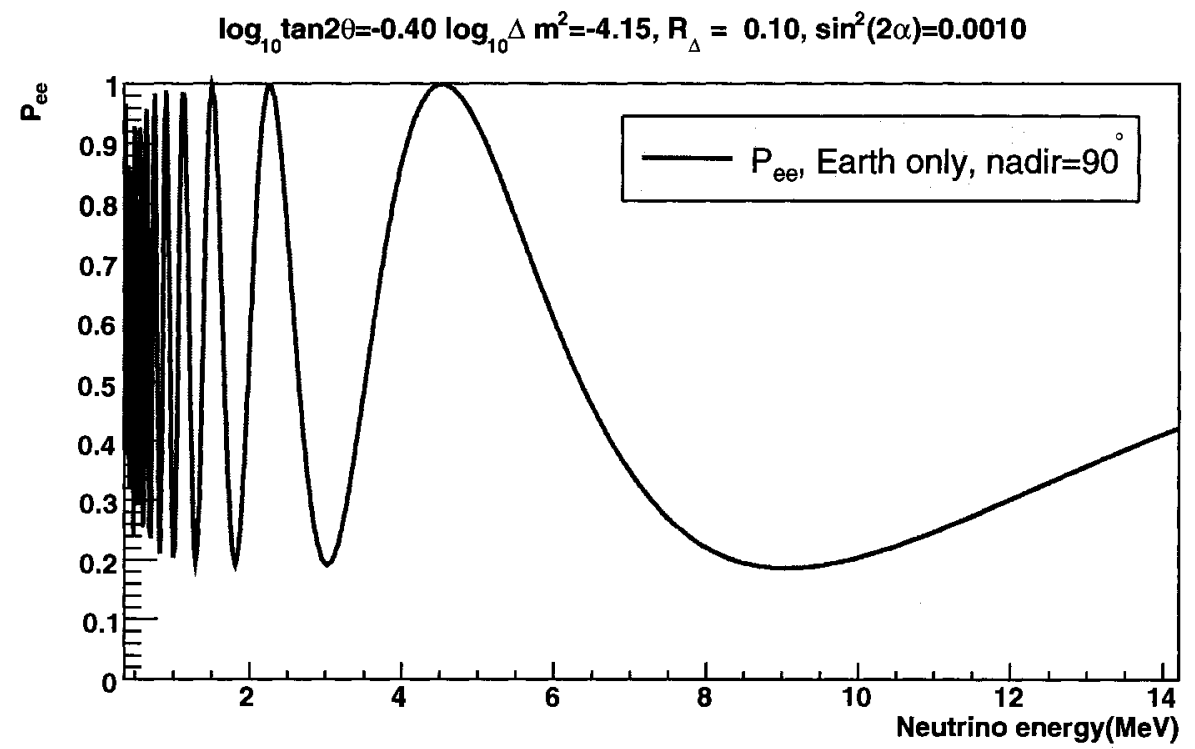

Figure 8.5: Survival probability $P_{e e}^{\ominus}$ for a pure electron neutrino state traversing the Earth at the nadir angle $\eta=90^{\circ}$. The sterile neutrino mixing parameters are $R_{\Delta}=0.10$ and $\sin ^{2} 2 \alpha=10^{-3}$. The LMA parameters are $\Delta m_{21}^{2}=7.1 \times 10^{-5} \mathrm{eV}^{2}$ and $\tan ^{2} \theta=0.40$.

Earth $\left(a_{\alpha}^{\beta}\right.$, where $\left.\alpha, \beta=s, e, a\right)$ are computed using Runge-Kutta [94] differential equation solver for 51 bins in $R_{\Delta} \in[0.0,0.25], 61$ bins in $\log \left(\sin ^{2} 2 \alpha\right) \in[-5,-2]$ and 91 bins in solar nadir angle $\eta \in\left[0^{\circ}, 180^{\circ}\right]$. The corresponding transition probabilities for the neutrino states are denoted as $P_{\alpha \beta}^{\ominus}$. An example of $P_{e e}^{\ominus}$ for the nadir angle $\eta=90^{\circ}$ is depicted in Figure 8.5. These probabilities are then averaged over the nadir angles, separately for day $\left(\eta>90^{\circ}\right)$, night $\left(\eta \leq 90^{\circ}\right)$ and $24 \mathrm{~h}$ period $\left(\eta \in\left[0^{\circ}, 180^{\circ}\right]\right)$ by using the zenith-binned livetime distribution for the experiments used in the analyses. Examples of averaged transition probabilities, assuming an ideal livetime for SNO, are shown in Figures 8.6, 8.7 and 8.8. 


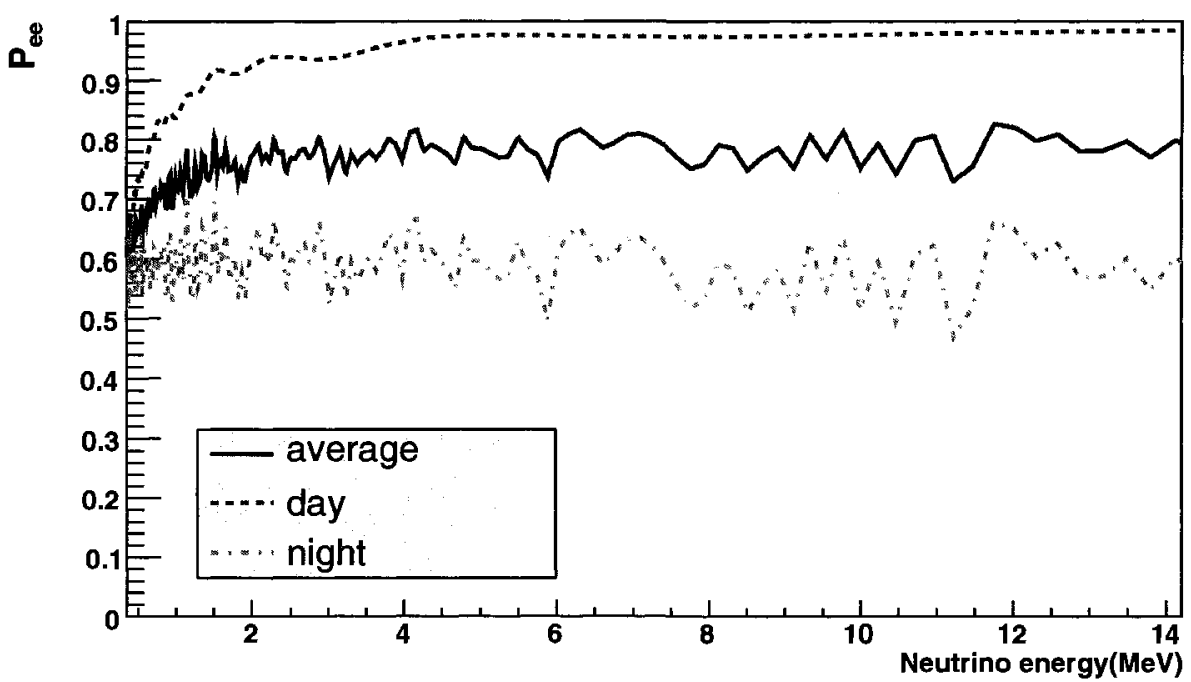

Figure 8.6: Survival probabilities $P_{e e}^{\ominus}$ for a pure electron neutrino state traversing the Earth for day (dotted), night (dashed) and day-nigh average (solid). The sterile neutrino mixing parameters are $R_{\Delta}=0.10$ and $\sin ^{2} 2 \alpha=10^{-3}$. The LMA parameters are $\Delta m_{21}^{2}=7.1 \times 10^{-5} \mathrm{eV}^{2}$ and $\tan ^{2} \theta=0.40$. 


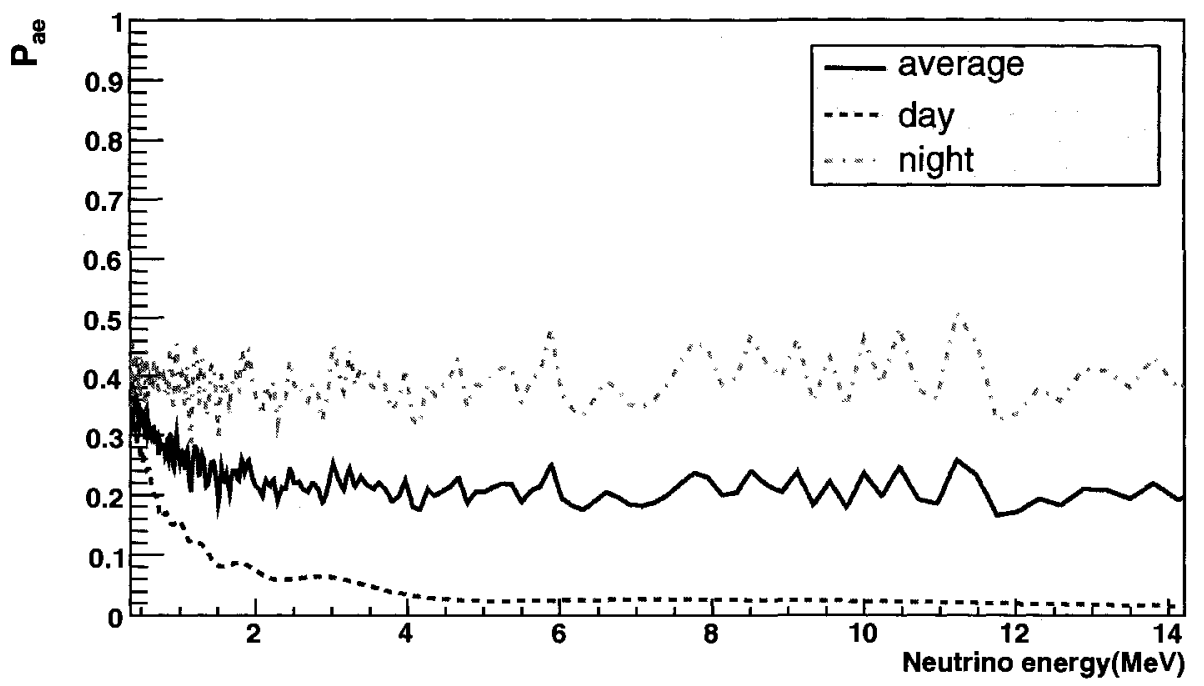

Figure 8.7: Transition probabilities $P_{a e}^{\ominus}$ for a pure active neutrino state traversing the Earth for day (dotted), night (dashed) and day-nigh average (solid). The sterile neutrino mixing parameters are $R_{\Delta}=0.10$ and $\sin ^{2} 2 \alpha=10^{-3}$. The LMA parameters are $\Delta m_{21}^{2}=7.1 \times 10^{-5} \mathrm{eV}^{2}$ and $\tan ^{2} \theta=0.40$. 


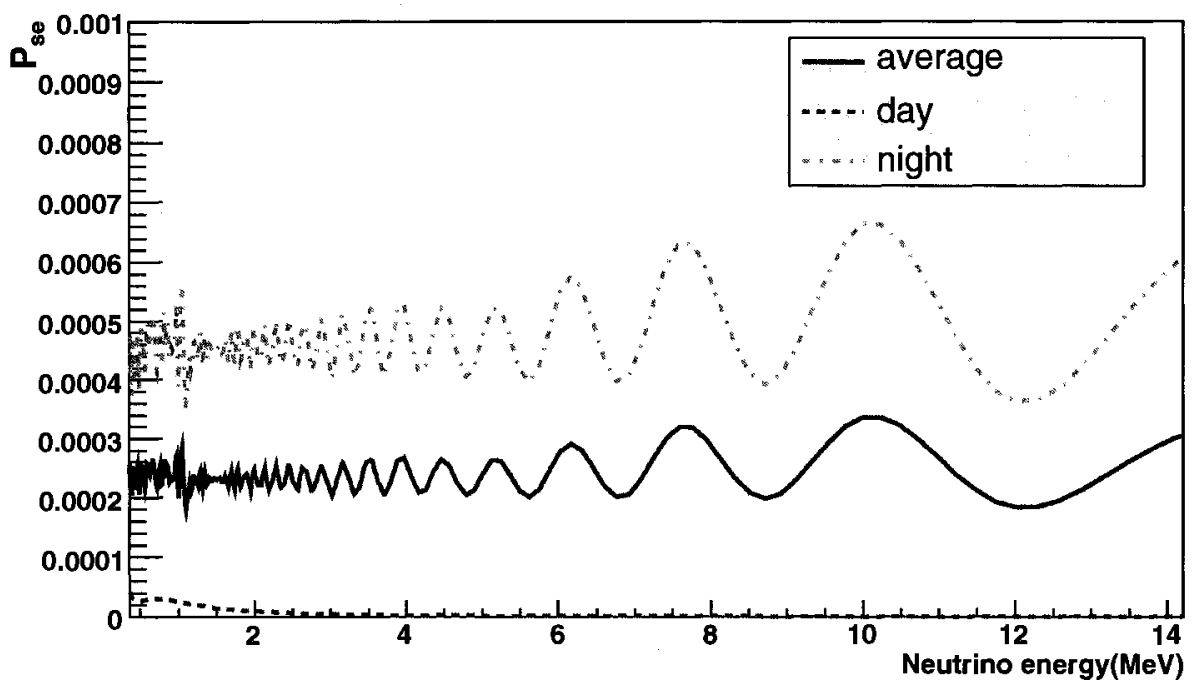

Figure 8.8: Transition probabilities $P_{s e}^{\ominus}$ for a pure sterile neutrino state traversing the Earth for day (dotted), night (dashed) and day-nigh average (solid). The sterile neutrino mixing parameters are $R_{\Delta}=0.10$ and $\sin ^{2} 2 \alpha=10^{-3}$. The LMA parameters are $\Delta m_{21}^{2}=7.1 \times 10^{-5} \mathrm{eV}^{2}$ and $\tan ^{2} \theta=0.40$. 


\section{Day-Night Effect}

After being averaged over nadir angles, the neutrino amplitudes $a_{\alpha}^{\beta}$ are combined with the solar neutrino amplitudes given in Equation (8.5), in order to calculate the final transition probabilities. In the weak eigenstate basis, a solar neutrino state arriving at the Earth's surface can be written as a linear combination of pure flavour states:

$$
\varphi(0)=\alpha_{s}\left|\nu_{s}\right\rangle+\alpha_{e}\left|\nu_{e}\right\rangle+\alpha_{a}\left|\nu_{a}\right\rangle .
$$

After propagating the distance $r$ inside the Earth, the solar neutrino state can be expressed in terms of pure flavour states given in Equations (8.10) and (8.11):

$$
\varphi(r)=\alpha_{s} \varphi_{s}(r)+\alpha_{e} \varphi_{e}(r)+\alpha_{a} \varphi_{a}(r) .
$$

The transition probabilities with the combined effects from neutrino propagation inside the Sun, vacuum and the Earth are therefore given as:

$$
\begin{aligned}
& P_{e e}^{\oplus}=\overline{\left|\left\langle\nu_{e} \mid \varphi(r)\right\rangle\right|^{2}}, \\
& P_{e s}^{\oplus}=\overline{\left|\left\langle\nu_{s} \mid \varphi(r)\right\rangle\right|^{2}}, \text { and } \\
& P_{e a}^{\oplus}=1-\left(P_{e e}^{\oplus}+P_{e s}^{\oplus}\right),
\end{aligned}
$$

where these expressions presented here in a compact form can be translated into the expressions that explicitly show contributions from the Sun and Earth transition amplitudes to the final probabilities:

$$
P_{e e}^{\oplus}=\bar{P}_{e s}^{\odot} P_{s e}^{\ominus}+\bar{P}_{e e}^{\odot} P_{e e}^{\ominus}+\bar{P}_{e a}^{\odot} P_{a e}^{\ominus}+2 \sum_{\delta=s, e, a} \bar{P}_{e \delta}^{\odot} \sum_{i=0}^{2} \sum_{\alpha \neq \beta} U_{\alpha i} U_{\beta i} a_{e}^{\alpha} a_{e}^{\beta}\left[\left|U_{i \beta}\right|^{2}\right]^{-1},
$$




$$
P_{e s}^{\oplus}=\bar{P}_{e s}^{\odot} P_{s s}^{\ominus}+\bar{P}_{e e}^{\odot} P_{e s}^{\ominus}+\bar{P}_{e a}^{\odot} P_{a s}^{\ominus}+2 \sum_{\delta=s, e, a} \bar{P}_{s \delta}^{\odot} \sum_{i=0}^{2} \sum_{\alpha \neq \beta} U_{\alpha i} U_{\beta i} a_{s}^{\alpha} a_{s}^{\beta}\left[\left|U_{i \beta}\right|^{2}\right]^{-1}
$$

where the transition probability $P_{e a}^{\oplus}$ is constrained to be the last expression in Equation (8.13).

Figure 8.9 depicts the electron neutrino survival probabilities $P_{e e}^{\oplus}$ for the ${ }^{8} \mathrm{~B}$ and hep neutrinos, separately averaged for day and for night using the livetime distribution for the SNO-I phase. It can be seen in Figure 8.9 that an electron neutrino has slightly higher survival probability during the night compared to that during day. The regeneration of electron neutrino flavour occurs at night, when neutrinos transverse the Earth when they are subjected to the MSW effect.

The transition probabilities described here are used to derive the model expectation for the solar neutrino yields at various detectors on Earth, with the data samples and uncertainties that have been summarized in Chapter 7. With the model predictions, an extraction of the sterile neutrino mixing parameters is performed and presented in the next section.

\subsection{Method}

The analyzed data samples from SNO are composed of summed kinetic energy spectra (CC+ES+NC+backgrounds) from SNO-I (day and night); CC kinetic energy spectra, ES and NC fluxes from SNO-II (day and night); and CC, ES and NC fluxes from SNO-III (averaged over day and night). These data samples are described in Chapter 6 (Section 6.1). Data from a partial SNO salt phase of running, that are composed of CC, ES and NC fluxes (averaged over day and night) are interpreted as well to benchmark our numerical calculation. This data 

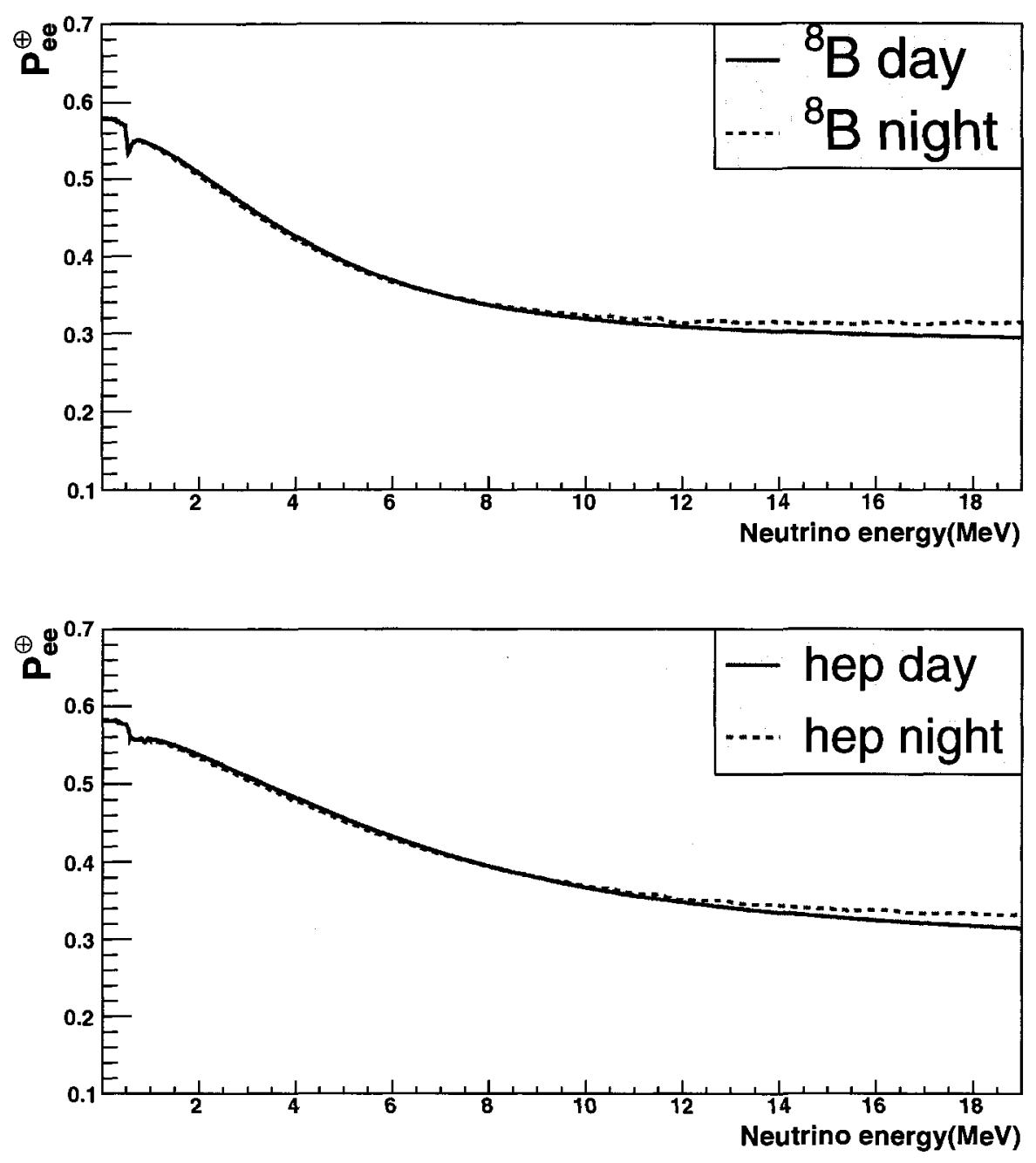

Figure 8.9: Survival probabilities $P_{e e}^{\oplus}$ for the ${ }^{8} \mathrm{~B}$ (top graph) and hep (bottom graph) neutrinos for the SNO-I phase, for both day (solid) and nigh (dotted). The sterile neutrino mixing parameters are $R_{\Delta}=0.05$ and $\sin ^{2} 2 \alpha=10^{-5}$. The LMA parameters used are $\Delta m_{21}^{2}=7.1 \times 10^{-5} \mathrm{eV}^{2}$ and $\tan ^{2} \theta=0.40$. 
sample can be found in Reference [73]. The measurements from other experiments used here are the Super-Kamiokande zenith binned energy spectra $[13,72]$ as well as the rate measurements from the Homestake [9], Gallex/GNO [10], SAGE [44] and Borexino [47].

A standard covariance $\chi^{2}$ approach is used in the analyses, as described by Equation (7.14). The expected yields of neutrino interactions obtained from the convolution of the detector response at each experiment are calculated with the prescription presented in Chapter 7. The survival and transition probabilities for an electron neutrino are obtained from the weakly mixed sterile neutrino model. The model expectations are inferred with the solar neutrino fluxes in the SSM BP00 [38] and BS05(OP) [40], for the analyses performed before and after SNOIII results, respectively. In the analyses, the flux of neutrinos from the ${ }^{8} \mathrm{~B}$ source is either a free parameter, or fixed to the SSM value, whereas the flux of hep neutrinos is always fixed to the SSM prediction. In the analyses before the SNOIII measurements, the ${ }^{8} \mathrm{~B}$ neutrino spectrum shape and its errors are taken from Ortiz et al. [85] and Bahcall et al. [86], respectively. After the SNO-III results, the ${ }^{8} \mathrm{~B}$ neutrino spectrum shape and its errors are taken from Winter et al. [63]. As explained before, the effects on energy related systematic uncertainties (energy scale, energy resolution and the ${ }^{8} \mathrm{~B}$ spectrum error) on the model expectations are derived at each point in the sterile neutrino mixing parameter space.

\subsection{The Results - SNO Only}

First, the data samples from the SNO-I and SNO-II phases are interpreted with the flux of the ${ }^{8} \mathrm{~B}$ neutrinos fixed to the SSM value of $\phi_{\mathrm{B}_{\mathrm{B}}}^{\mathrm{SSM}}=5.05 \times 10^{6} \mathrm{~cm}^{-2} \mathrm{~S}^{-1}$. The 
LMA parameters used are $\Delta m_{21}^{2}=7.1 \times 10^{-5} \mathrm{eV}^{2}$ and $\tan ^{2} \theta=0.40$. From "SNO-

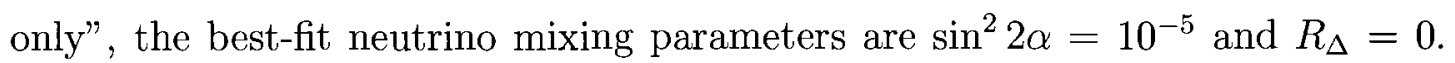
The minimum of the $\chi^{2}$ function is 74.40 for 70 degrees of freedom (dof). The inferred allowed regions in the $\sin ^{2} 2 \alpha-R_{\Delta}$ plane are depicted in Figure 8.10.

Secondly, the ${ }^{8} \mathrm{~B}$ neutrino flux is used as a free parameter. The extracted values for the mixing parameters are: $\sin ^{2} 2 \alpha=10^{-2}$ and $R_{\Delta}=0.01$. The minimum of the $\chi^{2}$ function is 74.24 for 69 dof and the fitted value for the flux of ${ }^{8} \mathrm{~B}$ neutrinos is $\phi_{8_{\mathrm{B}}}=5.23 \times 10^{6} \mathrm{~cm}^{-2} \mathrm{~s}^{-1}$. The corresponding confidence level contours in the $\sin ^{2} 2 \alpha-R_{\Delta}$ plane are shown in Figure 8.11.

The one dimensional projections of the $\Delta \chi^{2}$ function on the $\sin ^{2} 2 \alpha$ and $R_{\Delta}$ axes are depicted in Figure 8.12. An upper limit at 90\% CL on the parameter $R_{\Delta}$ can be placed, whereas no limit can be found on the sterile mixing angle. With the SNO data alone (SNO-I and SNO-II) $R_{\Delta}<0.13$ at the $90 \%$ CL when the ${ }^{8} \mathrm{~B}$ neutrino flux is fixed to the SSM expectation, and $R_{\Delta}<0.12$ at the $90 \% \mathrm{CL}$ when $\phi_{8_{\mathrm{B}}}$ is allowed to float. The limits obtained are summarized in Table 8.1 for both the ${ }^{8} \mathrm{~B}$ flux fixed and free analyses.

Table 8.1: Upper limits on the sterile neutrino mixing parameters at 90\% CL from the SNO-I+SNO-II (partial) oscillation analyses.

\begin{tabular}{lcc}
\hline \hline Parameter & SSM $^{8} \mathrm{~B}$ flux & Free \\
& & $\mathrm{B}$ flux \\
\hline $\sin ^{2} 2 \alpha$ & no limit at $90 \% \mathrm{CL}$ \\
$R_{\Delta}$ & 0.13 & 0.12 \\
\hline \hline
\end{tabular}

With the addition of the SNO-III measurements, the data samples from all three phases are interpreted first with the ${ }^{8} \mathrm{~B}$ neutrino flux fixed to the SSM value of $\phi_{8_{\mathrm{B}}}^{\mathrm{SSM}}=5.69 \times 10^{6} \mathrm{~cm}^{-2} \mathrm{~S}^{-1}$. The LMA parameters remained unchanged 


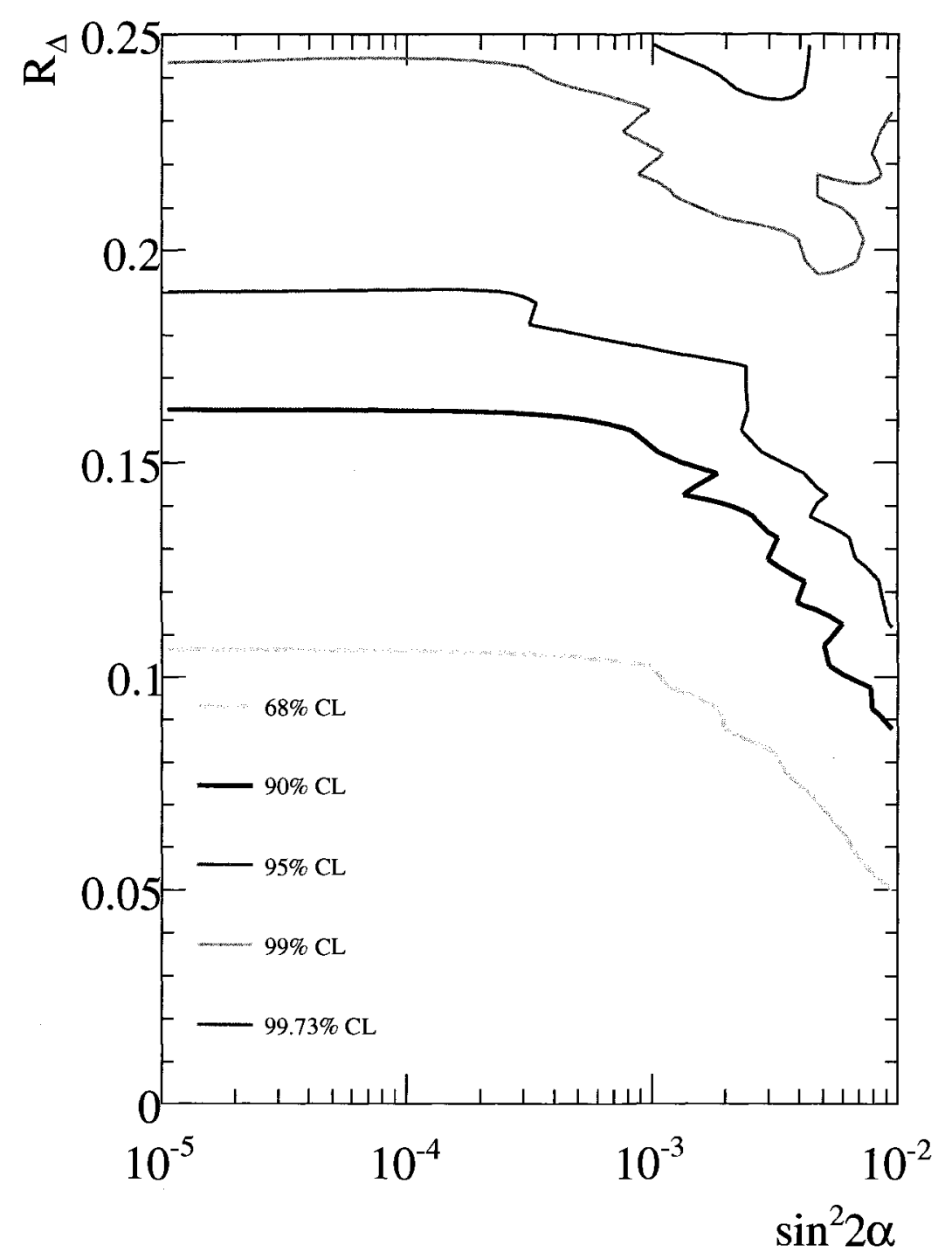

Figure 8.10: Before SNO-III: SNO-only $\chi^{2}$ confidence level contours on the sterile neutrino parameter space. This analysis includes SNO-I summed kinetic energy spectra (day and night); SNO-II NC, ES fluxes, and CC spectra (day and night). The best fit point is at: $\sin ^{2} 2 \alpha=10^{-5}, R_{\Delta}=0$. The fluxes of ${ }^{8} \mathrm{~B}$ and hep neutrinos are fixed at $5.05 \times 10^{6} \mathrm{~cm}^{-2} \mathrm{~s}^{-1}$ and $9.3 \times 10^{3} \mathrm{~cm}^{-2} \mathrm{~s}^{-1}$, respectively. The LMA parameters used are $\Delta m_{21}^{2}=7.1 \times 10^{-5} \mathrm{eV}^{2}$ and $\tan ^{2} \theta=0.40$. 


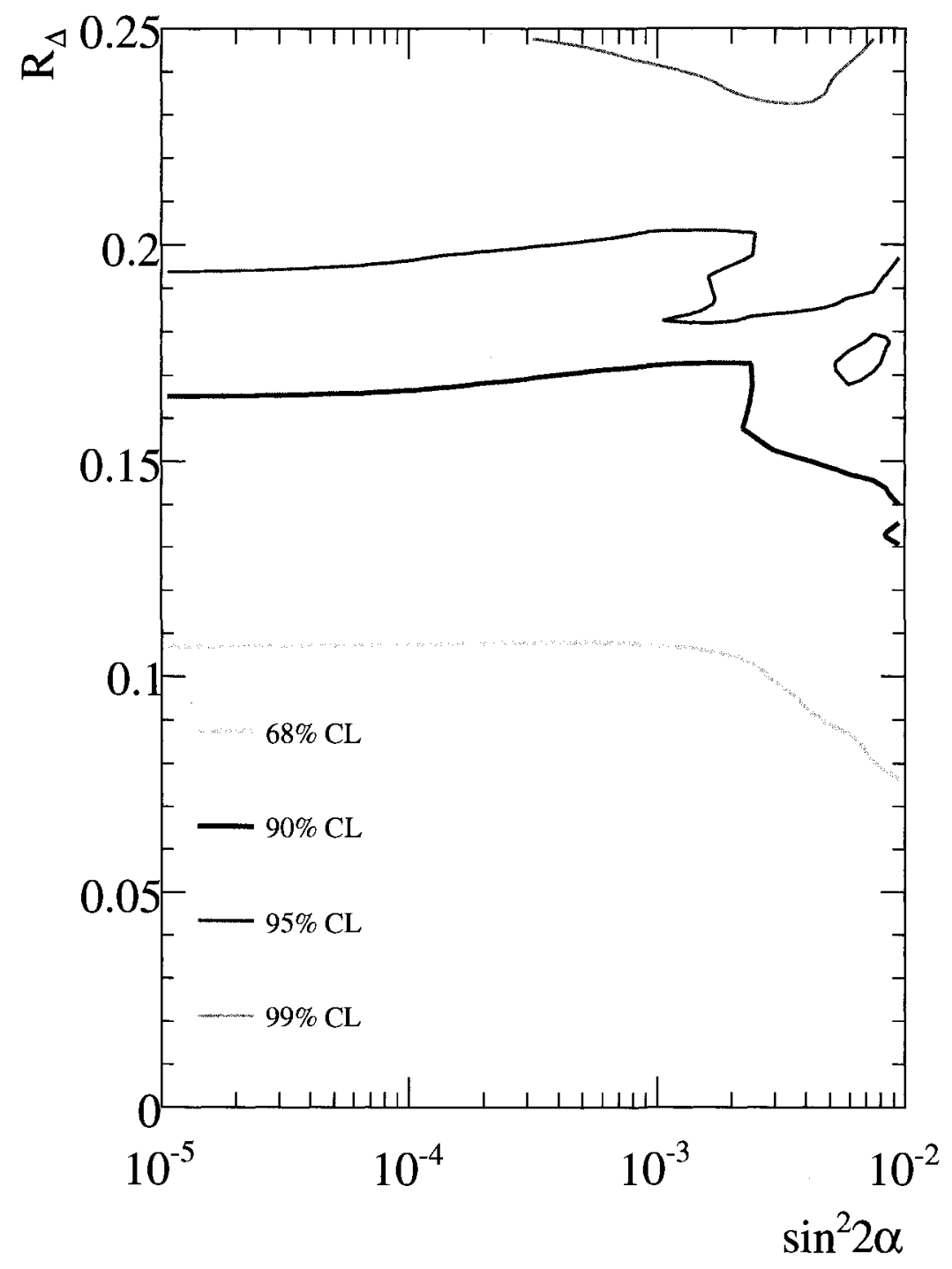

Figure 8.11: Before SNO-III: SNO-only $\chi^{2}$ confidence level contours in the sterile neutrino parameter space. This analysis includes SNO-I summed kinetic energy spectra (day and night); SNO-II NC, ES fluxes, and CC spectra (day and night). The best fit point is at $\sin ^{2} 2 \alpha=10^{-2}, R_{\Delta}=0.01$ and $\phi_{8_{\mathrm{B}}}=5.23 \times 10^{6} \mathrm{~cm}^{-2} \mathrm{~s}^{-1}$. The hep flux is fixed at $9.3 \times 10^{3} \mathrm{~cm}^{-2} \mathrm{~s}^{-1}$. The LMA parameters used are $\Delta m_{21}^{2}=$ $7.1 \times 10^{-5} \mathrm{eV}^{2}, \tan ^{2} \theta=0.40$. 

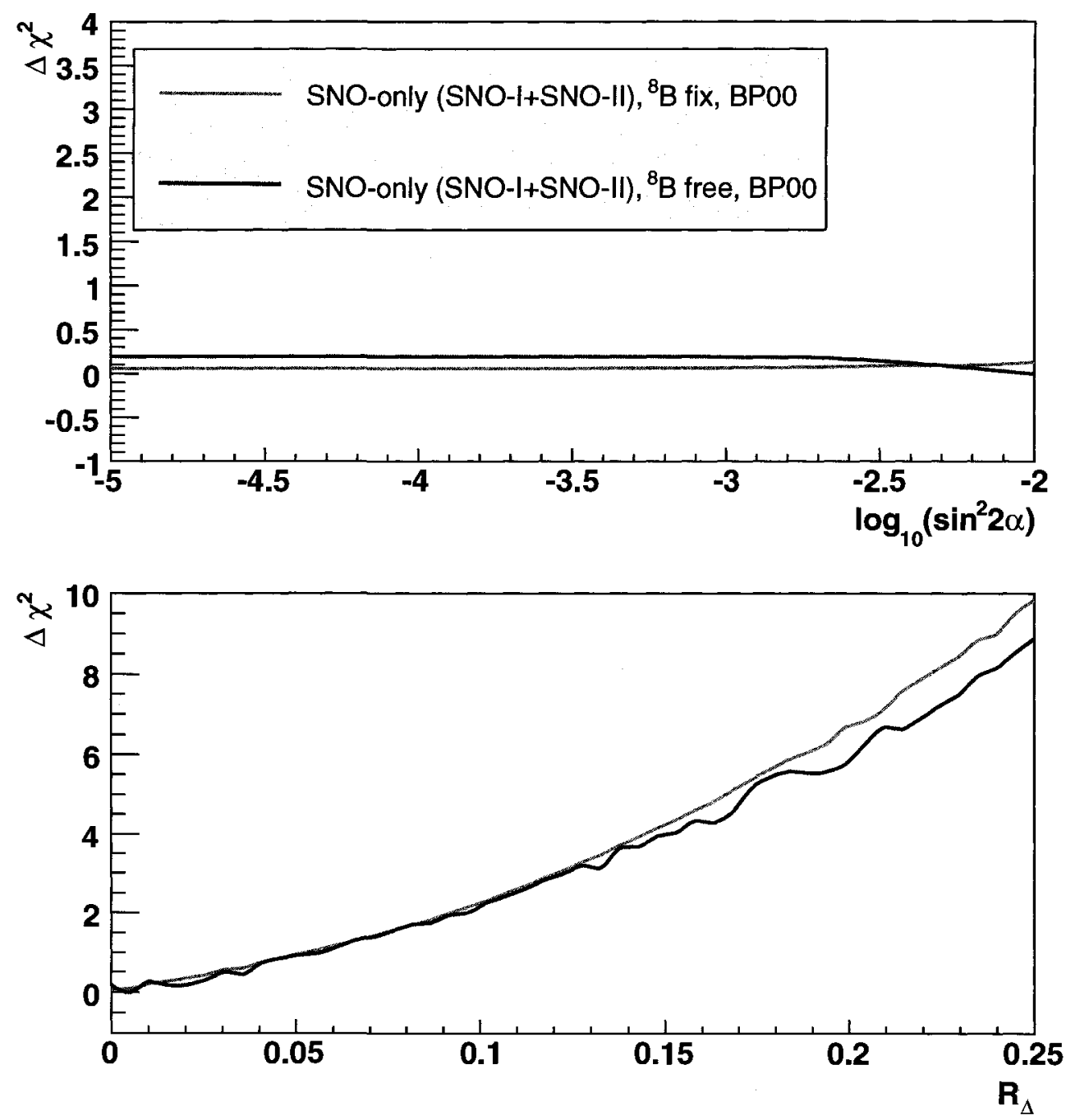

Figure 8.12: Before SNO-III: One dimensional projections of the $\Delta \chi^{2}$ function on the $\sin ^{2} 2 \alpha$ and $R_{\Delta}$ axis from the SNO-only fit. 
from the analysis performed before the inclusion of the SNO-III results. The extracted sterile neutrino mixing parameters are $\sin ^{2} 2 \alpha=10^{-2}$ and $R_{\Delta}=0.01$, at the minimum of the $\chi^{2}$ function of 76.64 for 73 dof. The corresponding allowed regions in the $\sin ^{2} 2 \theta-R_{\Delta}$ plane are depicted in Figure 8.13.

Finally, the full data samples from SNO-I, SNO-II and SNO-III phases are interpreted with the floating ${ }^{8} \mathrm{~B}$ neutrino flux. This analysis yields $\sin ^{2} 2 \alpha=10^{-2}$, $R_{\Delta}=0.01$ and $\phi_{\mathrm{B}_{\mathrm{B}}}=5.31 \times 10^{-6} \mathrm{~cm}^{-2} \mathrm{~s}^{-1}$. The minimum of the $\chi^{2}$ function is 76.87 for 72 dof. The confidence level contours in the $\sin ^{2} 2 \theta-R_{\Delta}$ plane are shown in Figure 8.14.

The one dimensional projections of the $\Delta \chi^{2}$ function on the $\sin ^{2} 2 \alpha$ and $R_{\Delta}$ axes are snown in Figure 8.15. Again, an upper limit at 90\% CL on the parameter $R_{\Delta}$ can be placed, whereas no limit can be found on the sterile mixing angle. With the SNO data alone (SNO-I, SNO-II and SNO-III) $R_{\Delta}<0.11$ at the $90 \%$ CL for both the ${ }^{8} \mathrm{~B}$ neutrino flux fixed and free analyses. These results are summarized in Table 8.2 .

Table 8.2: Upper limits on the sterile neutrino mixing parameters at 90\% CL from the SNO-only (SNO-I+SNO-II+SNO-III) oscillation analyses.

\begin{tabular}{lcc}
\hline \hline Parameter & SSM $^{8} \mathrm{~B}$ flux & Free ${ }^{8} \mathrm{~B}$ flux \\
\hline $\sin ^{2} 2 \alpha$ & no limit at & $90 \%$ CL \\
$R_{\Delta}$ & 0.11 & 0.11 \\
\hline \hline
\end{tabular}

Thus the data samples from SNO-only (SNO-I+SNO-II+SNO-III) are not sufficient to constrain the sterile mixing angle, whereas the upper limit on the parameter $R_{\Delta}$ is found to be 0.11 at the $90 \% \mathrm{CL}$ for both the fixed and free ${ }^{8} \mathrm{~B}$ neutrino flux. The limits on the mixing angle $\sin ^{2} 2 \alpha$ cannot be placed because of 


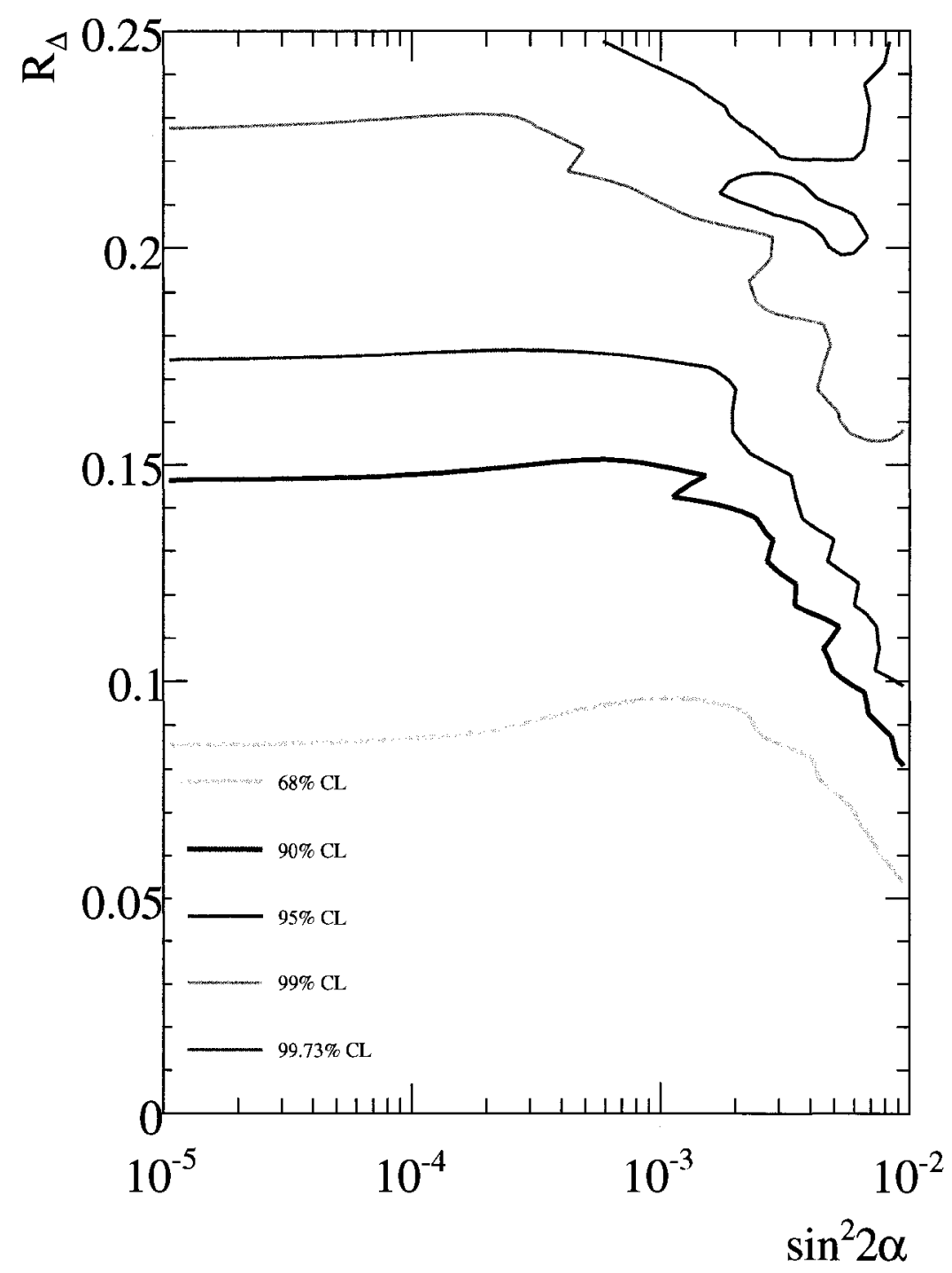

Figure 8.13: After SNO-III: SNO-only $\chi^{2}$ confidence level contours on the sterile neutrino parameter space. This analysis includes SNO-I summed kinetic energy spectra (day and night); SNO-II NC, ES fluxes, and CC spectra (day and night); and SNO-III CC, ES and NC fluxes (averaged). The best fit point is at: $\sin ^{2} 2 \alpha=$ $10^{-2}, R_{\Delta}=0.01$. The fluxes of ${ }^{8} \mathrm{~B}$ and hep neutrinos are fixed at $5.69 \times 10^{6}$ $\mathrm{cm}^{-2} \mathrm{~s}^{-1}$ and $7.93 \times 10^{3} \mathrm{~cm}^{-2} \mathrm{~s}^{-1}$, respectively. The LMA parameters used are $\Delta m_{21}^{2}=7.1 \times 10^{-5} \mathrm{eV}^{2}$ and $\tan ^{2} \theta=0.40$. 


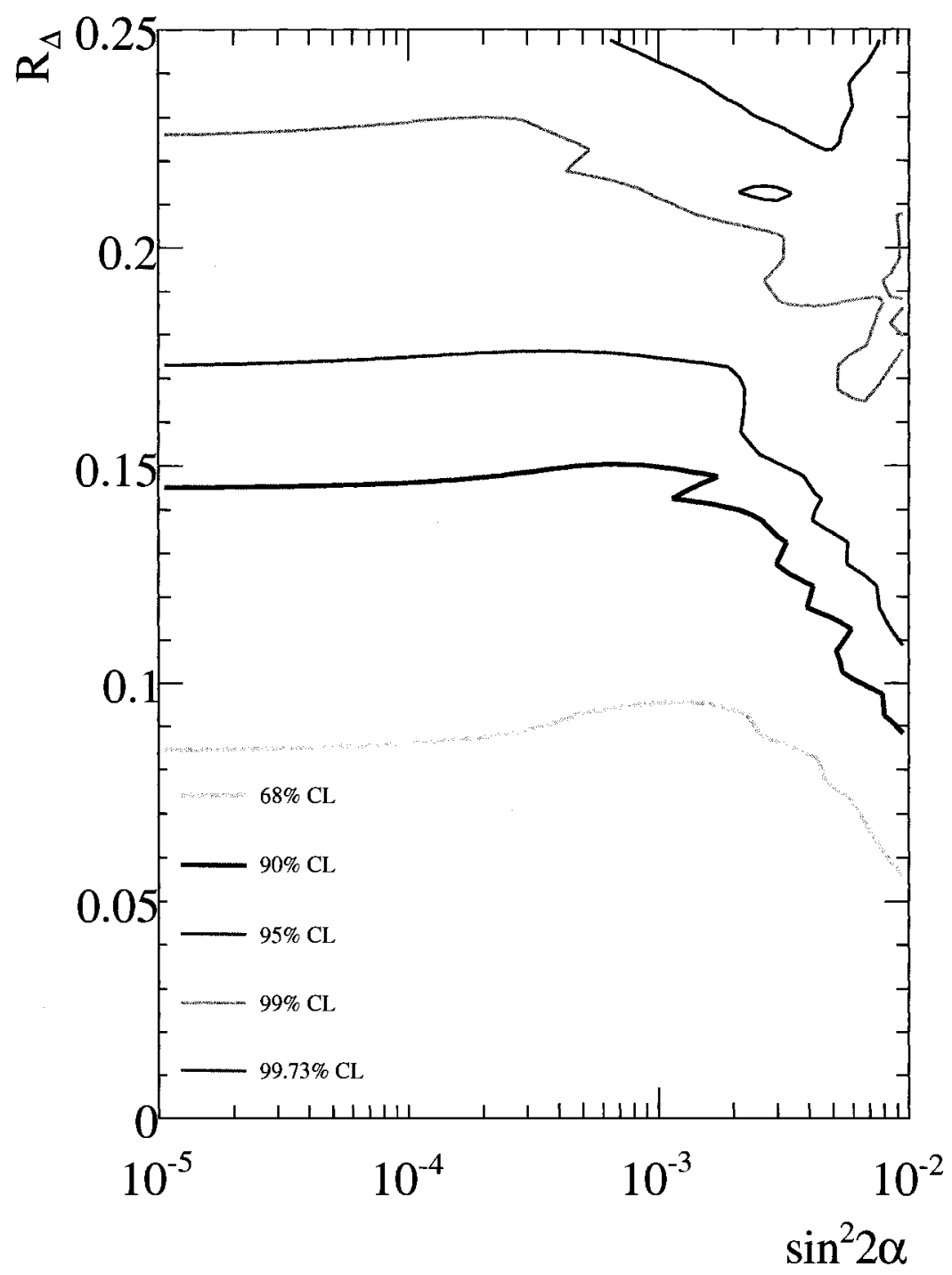

Figure 8.14: After SNO-III: SNO-only $\chi^{2}$ confidence level contours on the sterile neutrino parameter space. This analysis includes SNO-I summed kinetic energy spectra (day and night); SNO-II NC, ES fluxes, and CC spectra (day and night); and SNO-III CC, ES and NC fluxes (averaged). The best fit point is at: $\sin ^{2} 2 \alpha=$ $10^{-2}, R_{\Delta}=0.01$ and $\phi_{8_{\mathrm{B}}}=5.31 \times 10^{-6} \mathrm{~cm}^{-2} \mathrm{~s}^{-1}$. The hep neutrino flux is fixed at $7.93 \times 10^{3} \mathrm{~cm}^{-2} \mathrm{~s}^{-1}$. The LMA parameters used are $\Delta m_{21}^{2}=7.1 \times 10^{-5} \mathrm{eV}^{2}$ and $\tan ^{2} \theta=0.40$. 

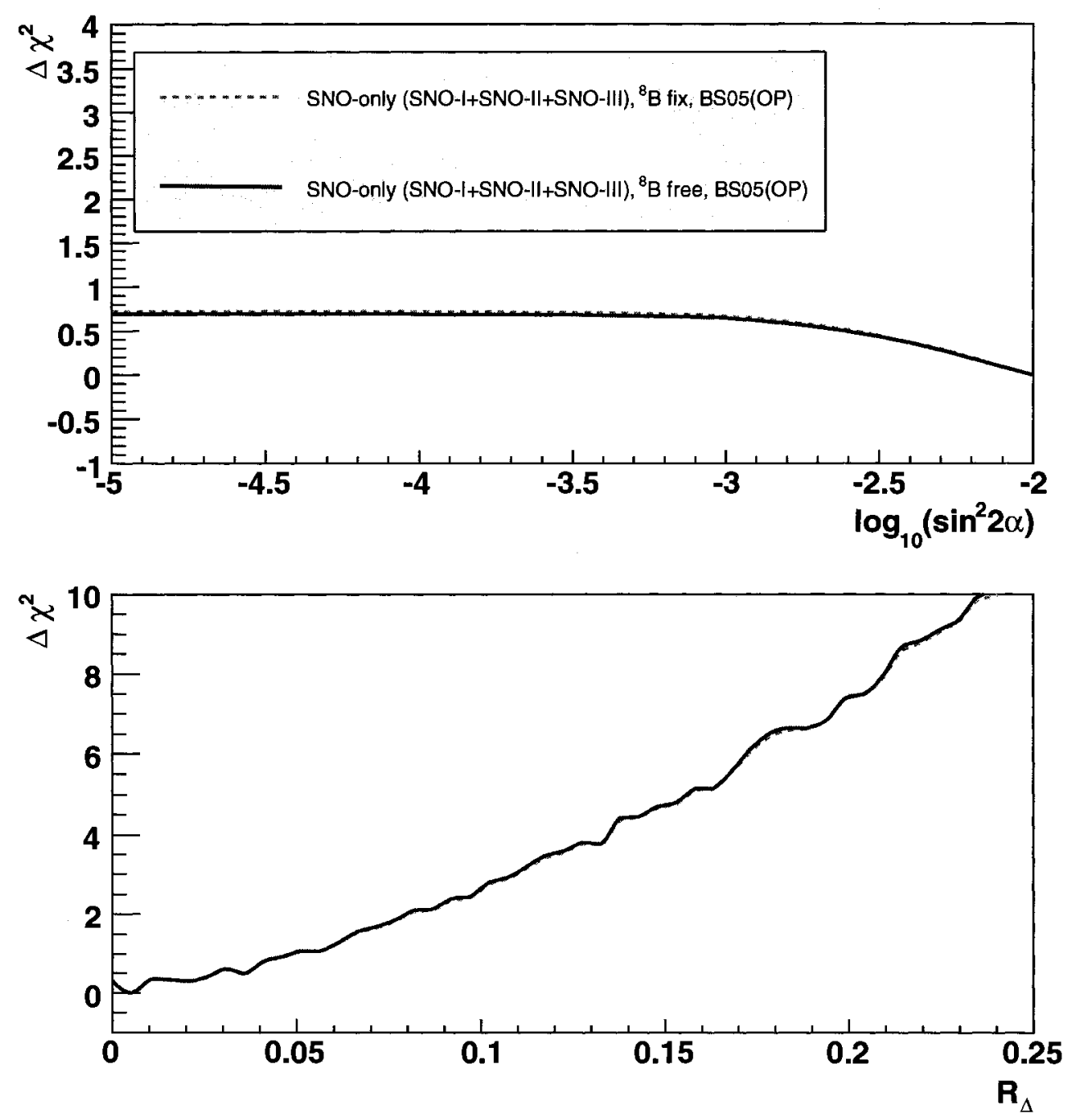

Figure 8.15: After SNO-III: One dimensional projections of the $\Delta \chi^{2}$ function on the $\sin ^{2} 2 \alpha$ and $R_{\Delta}$ axis from the SNO-only fit. 
rather high electron kinetic energy thresholds at SNO, whereas the main impact of a sterile neutrino is expected to be at lower energies.

\subsection{Solar Global Results}

The interpretation of the global solar data with the results from SNO is performed three times. At first, the data sample from SNO-I and the partial SNO salt phase are added to the global $\chi^{2}$ function and tested against the model. This test is performed in order to compare the best fitted values for the sterile mixing parameters to the results of Holanda and Smirnov in Reference [30]. Our analysis is a full numerical scan of the sterile mixing parameter; while the analysis presented in Reference [30] study the effect at discrete value of $\sin ^{2} 2 \alpha$. The objective of the fit to only the partial SNO-II data sample was to benchmark the full numerical procedure described in Section 8.2. After that, the complete data set from the SNO-II phase is added to the analysis. Finally, after the SNO-III results, the interpretation is performed by adding the SNO-III CC, ES and NC fluxes, the latest Borexino [47] measurement and updated zenith energy spectra from SK-I [72].

\subsubsection{Results - Partial SNO-II}

Here, the partial SNO-II is referred to as the analysis published on September $7^{\text {th }}, 2003$ in Reference [73] where CC, ES and NC fluxes were obtained with 254.2 live days of the salt phase. From the analysis of solar global data with the partial data sample from the SNO-II phase, the allowed regions in $\sin ^{2} 2 \alpha-R_{\Delta}$ plane are shown in Figures 8.16 and 8.17 for the fixed and floated ${ }^{8} \mathrm{~B}$ neutrino flux, respectively. The first significant feature from these results is a highly excluded 
area at the low values of the ratio $R_{\Delta}$ and large mixing angles (bottom-right areas in the Figures 8.16 and 8.17 ). This area is excluded mainly by the radiochemical experiments that measure fluxes of the low-energy neutrinos, because for larger $\sin ^{2} 2 \alpha$ and smaller $R_{\Delta}$ values, the position of the dip in the survival probabilities is positioned at lower neutrino energies. The second excluded region is at the high values of the ratio $R_{\Delta}$ and large mixing angles. This region is manly excluded with the data from SNO and SK, since the dip in electron neutrino survival probabilities is large and shifted towards a higher energy range, that SNO and SK are mostly sensitive to.

With the SSM flux of ${ }^{8} \mathrm{~B}$ neutrinos, the extracted mixing parameters are: $\sin ^{2} 2 \alpha=7.1_{-6.9}^{+16.0} \times 10^{-4}$ and $R_{\Delta}=0.090_{-0.031}^{+0.036}$. The minimum of the $\chi^{2}$ is achieved at 72.57 for number of degrees of freedom of 82 . From the analysis with the flux of ${ }^{8} \mathrm{~B}$ neutrinos as a free parameter, the extracted values for the mixing parameters are: $\sin ^{2} 2 \alpha=7.9_{-7.7}^{+17.2} \times 10^{-4}$ and $R_{\Delta}=0.090_{-0.025}^{+0.046}$. The minimum of the $\chi^{2}$ is at 72.28 for $81 d o f$ and the flux of the ${ }^{8} \mathrm{~B}$ neutrinos is found to be $\phi_{8_{\mathrm{B}}}=5.28 \times 10^{6} \mathrm{~cm}^{-2} \mathrm{~s}^{-1}$. The upper limits on the sterile mixing angle are set at $\sin ^{2} 2 \alpha<4.0 \times 10^{-3}$ and $\sin ^{2} 2 \alpha<4.5 \times 10^{-3}$ at $90 \% \mathrm{CL}$, for the ${ }^{8} \mathrm{~B}$ neutrino flux fixed and free analyses, respectively.

The results from the two analyses are summarized in Table 8.3. These results of SNO-I and the partial SNO-II are in perfect agreement with the prediction from Holanda and Smirnov [30] that yields $\sin ^{2} 2 \alpha \approx 10^{-3}$ and $R_{\Delta}=0.09$ and validate our calculations. Our full numerical method can then be used for the full SNO-II and SNO-III data. 


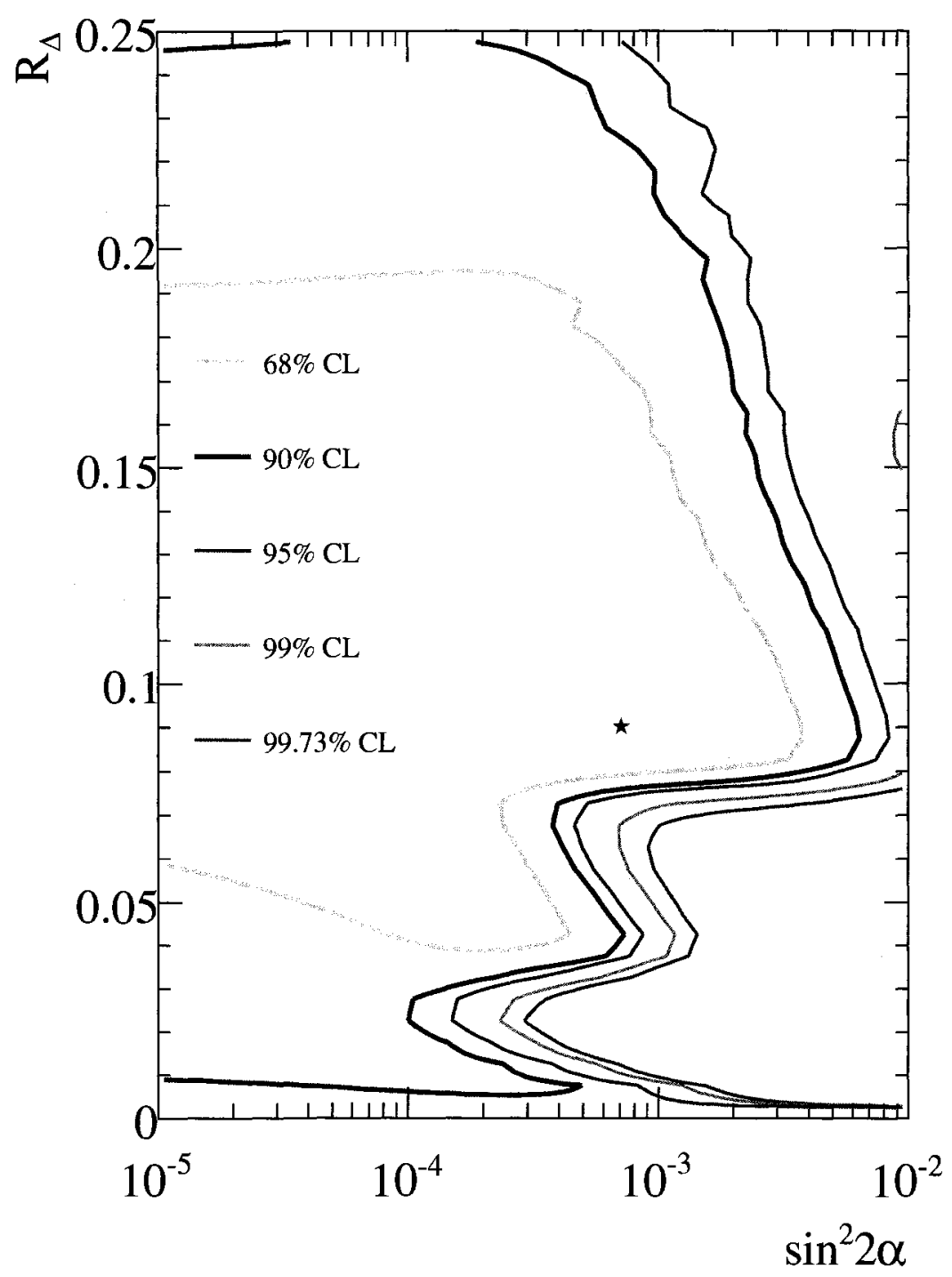

Figure 8.16: Global $\chi^{2}$ confidence level contours in the sterile neutrino parameter space. This analysis includes SNO-I summed kinetic energy spectra (day and night); CC, NC and ES fluxes from the partial SNO-II phase (day-night average); SK zenith binned kinetic energy spectra; and the rate measurements from the Homestake, Gallex/GNO and SAGE. The best-fit point is at: $\sin ^{2} 2 \alpha=7.1 \times 10^{-4}$, $R_{\Delta}=0.09$ with $\chi_{\min }^{2}=72.57 / 82($ dof $)$. The ${ }^{8} \mathrm{~B}$ neutrino flux was fixed to $5.05 \times 10^{6}$ $\mathrm{cm}^{-2} \mathrm{~s}^{-1}$. The LMA parameters used are $\Delta m_{21}^{2}=7.1 \times 10^{-5} \mathrm{eV}^{2}$ and $\tan ^{2} \theta=0.40$. 


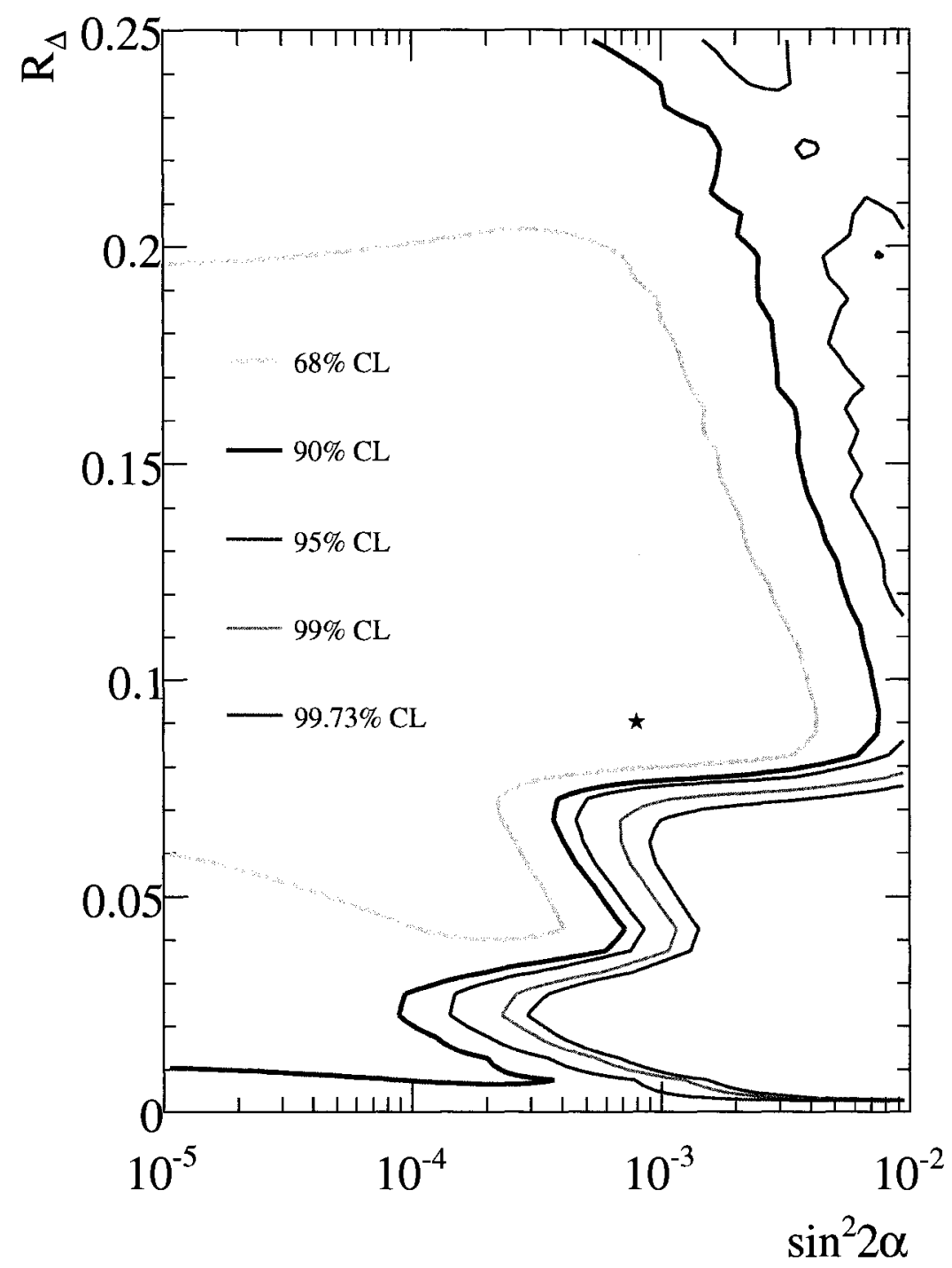

Figure 8.17: Global $\chi^{2}$ confidence level contours in sterile neutrino parameter space. This analysis includes SNO-I summed kinetic energy spectra (day and night); CC, NC and ES fluxes from the partial SNO-II phase (day-night average); SK zenith binned kinetic energy spectra; and the rate measurements from the Homestake, Gallex/GNO and SAGE. The best-fit point is at $\sin ^{2} 2 \alpha=7.9 \times 10^{-4}$, $R_{\Delta}=0.09$ and $\phi_{8_{\mathrm{B}}}=5.28 \times 10^{6} \mathrm{~cm}^{-2} \mathrm{~s}^{-1}$ with $\chi_{\min }^{2}=72.28 / 81($ dof $)$. The LMA parameters used are $\Delta m_{21}^{2}=7.1 \times 10^{-5} \mathrm{eV}^{2}$ and $\tan ^{2} \theta=0.40$. 


\subsubsection{Results - Full SNO-II}

The full salt data sample correspond to 391 live days where CC spectra as well as the ES and NC fluxes were measured for both day and night. The best-fit results from the global solar analyses with the full data sample from the SNO-II phase

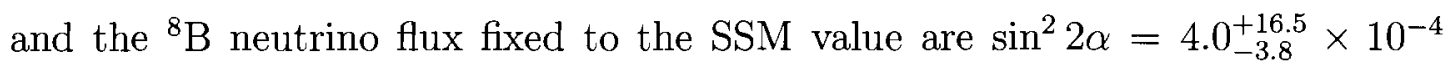
and $R_{\Delta}=0.080_{-0.029}^{+0.037}$. The best-fit is achieved at the minimum value of the $\chi^{2}$ function of 119.41 for 117 dof. By interpreting the data with the ${ }^{8} \mathrm{~B}$ neutrino flux as a free parameter, the best-fit values are found to be: $\sin ^{2} 2 \alpha=8.9_{-8.6}^{+18.2} \times 10^{-4}$ and $R_{\Delta}=0.090_{-0.027}^{+0.030}$ at the minimum $\chi^{2}$ of 118.76 for 116 dof. The fitted ${ }^{8} \mathrm{~B}$ neutrino flux is $\phi_{8 \mathrm{~B}}=5.32 \times 10^{6} \mathrm{~cm}^{-2} \mathrm{~s}^{-1}$. At $90 \% \mathrm{CL}$, the derived upper limits on the sterile mixing angle are $\sin ^{2} 2 \alpha<3.6 \times 10^{-3}$ and $\sin ^{2} 2 \alpha<4.9 \times 10^{-3}$, for the ${ }^{8} \mathrm{~B}$ neutrino flux fixed and free analyses, respectively. Table 8.3 summarizes the results.

Table 8.3: Global solar best-fit parameters with the partial and full data samples from the SNO-II phase. The ${ }^{8} \mathrm{~B}$ neutrino fluxes are expressed in units of $10^{6} \mathrm{~cm}^{-2}$ $\mathrm{s}^{-1}$. All fits are performed with $\Delta m_{21}^{2}=7.1 \times 10^{-5} \mathrm{eV}^{2}$ and $\tan ^{2} \theta=0.40$.

\begin{tabular}{lll}
\hline \hline Parameter & SSM $^{8} \overline{\mathrm{B} \text { flux }}$ & Free \\
\hline & \multicolumn{1}{c}{ Partial dux } \\
\hline $\sin ^{2} 2 \alpha$ & $<4.0 \times 10^{-3}$ at $90 \% \mathrm{CL} \quad<4.5 \times 10^{-3}$ at $90 \% \mathrm{CL}$ \\
$R_{\Delta}$ & $0.090_{-0.031}^{+0.036}$ & $0.090_{-0.025}^{+0.046}$ \\
$\phi_{8_{\mathrm{B}}}$ & 5.05 & 5.28 \\
\hline \multicolumn{3}{c}{ Full data sample from SNO-II } \\
\hline $\sin ^{2} 2 \alpha$ & $<3.6 \times 10^{-3}$ at $90 \% \mathrm{CL} \quad<4.9 \times 10^{-3}$ at $90 \% \mathrm{CL}$ \\
$R_{\Delta}$ & $0.080_{-0.029}^{+0.037}$ & $0.090_{-0.027}^{+0.030}$ \\
$\phi_{\varepsilon_{\mathrm{B}}}$ & 5.05 & 5.32 \\
\hline \hline
\end{tabular}

The confidence level contours in the $\sin ^{2} 2 \alpha-R_{\Delta}$ plane are depicted in Figures 8.18 and 8.19 , with the fixed and fitted ${ }^{8} \mathrm{~B}$ neutrino fluxes, respectively. When 
compared to the previous results obtained with the partial data set from the SNO-II phase (Figures 8.18 and 8.19), improvements are observed in the allowed regions. In the former analysis, the $\mathrm{CC}, \mathrm{ES}$ and $\mathrm{NC}$ fluxes that contributed to the constraint of the sterile model were averaged over day and night. Whereas in the latter analyses, the $\mathrm{CC}$ spectra as well as the $\mathrm{NC}$ and $\mathrm{CC}$ fluxes for both day and night are used. The spectral information from the CC signal and the day-night information further constrain the allowed regions. In addition, the best fit point is shifted towards smaller values of the mixing parameters when the ${ }^{8} \mathrm{~B}$ neutrino flux is fixed and towards a higher $\sin ^{2} 2 \alpha$ for the free ${ }^{8} \mathrm{~B}$ neutrino flux analysis. The one dimensional projections of the global $\Delta \chi^{2}$ functions, from which the errors on the extracted parameter are inferred, are depicted in Figure 8.20.

\subsubsection{Results - After SNO-III}

The latest global solar neutrino analysis is performed by adding the results from the SNO-III phase, the updated results from SK-I [72] and latest Borexino measurement [47]. The model predictions are recalculated for all neutrino experiments by using the SSM BS05(OP) [40] and ${ }^{8} \mathrm{~B}$ neutrino spectrum shape from Winter et al. [63].

The best-fit results from the ${ }^{8} \mathrm{~B}$ neutrino flux fixed to the SSM value are $\sin ^{2} 2 \alpha=2.2_{-1.4}^{+2.3} \times 10^{-3}$ and $R_{\Delta}=0.10_{-0.02}^{+0.04}$. The best-fit is achieved at the minimum value of the $\chi^{2}$ function of 132.12 for 121 dof. Interpretation of the data with the ${ }^{8} \mathrm{~B}$ neutrino flux as a free parameter yields: $\sin ^{2} 2 \alpha=2.8_{-1.6}^{+2.9} \times 10^{-3}$ and $R_{\Delta}=0.11_{-0.03}^{+0.04}$ at the minimum $\chi^{2}$ of 132.24 for 120 dof. The fitted ${ }^{8} \mathrm{~B}$ neutrino flux is $\phi_{8_{\mathrm{B}}}=5.66 \times 10^{6} \mathrm{~cm}^{-2} \mathrm{~s}^{-1}$. At $90 \% \mathrm{CL}$, the derived upper limits on the sterile mixing angle are $\sin ^{2} 2 \alpha<7.7 \times 10^{-3}$ and $\sin ^{2} 2 \alpha<9.9 \times 10^{-3}$, for the ${ }^{8} \mathrm{~B}$ 


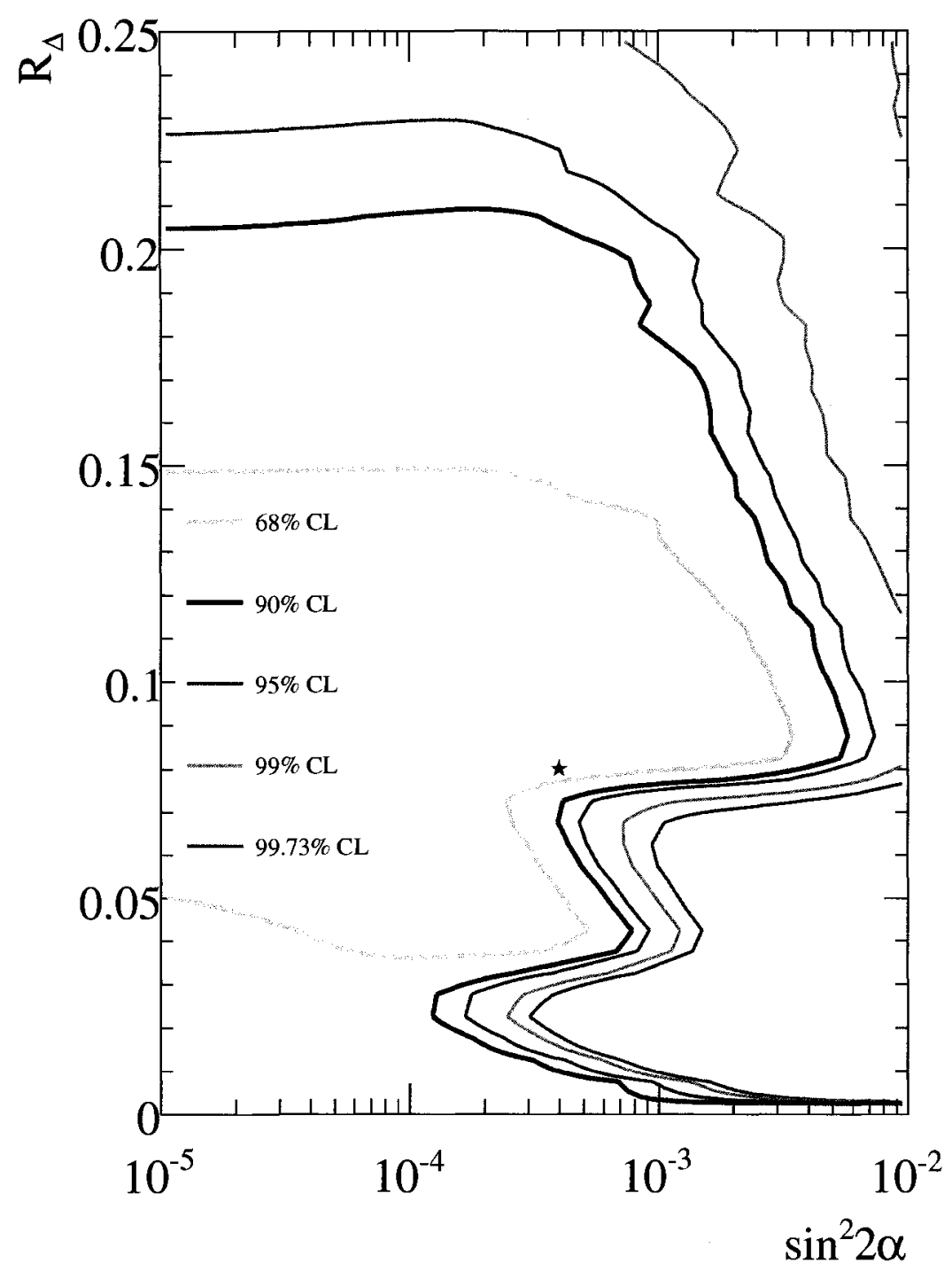

Figure 8.18: Global $\chi^{2}$ confidence level contours of the sterile neutrino parameter space. This analysis includes SNO-I summed kinetic energy spectra (day and night); SNO-II CC kinetic energy spectra, NC and ES fluxes (day and night); SK zenith binned kinetic energy spectra; and the rate measurements from the Homestake, Gallex/GNO and SAGE. The best fit point is at $\sin ^{2} 2 \alpha=4.0 \times 10^{-4}$ and $R_{\Delta}=0.08$ with $\chi_{\min }^{2}=119.41 / 117($ dof $)$. The ${ }^{8} \mathrm{~B}$ neutrino flux was fixed to $5.05 \times 10^{6} \mathrm{~cm}^{-2} \mathrm{~s}^{-1}$. The LMA parameters used are $\Delta m_{21}^{2}=7.1 \times 10^{-5} \mathrm{eV}^{2}$ and $\tan ^{2} \theta=0.40$. 


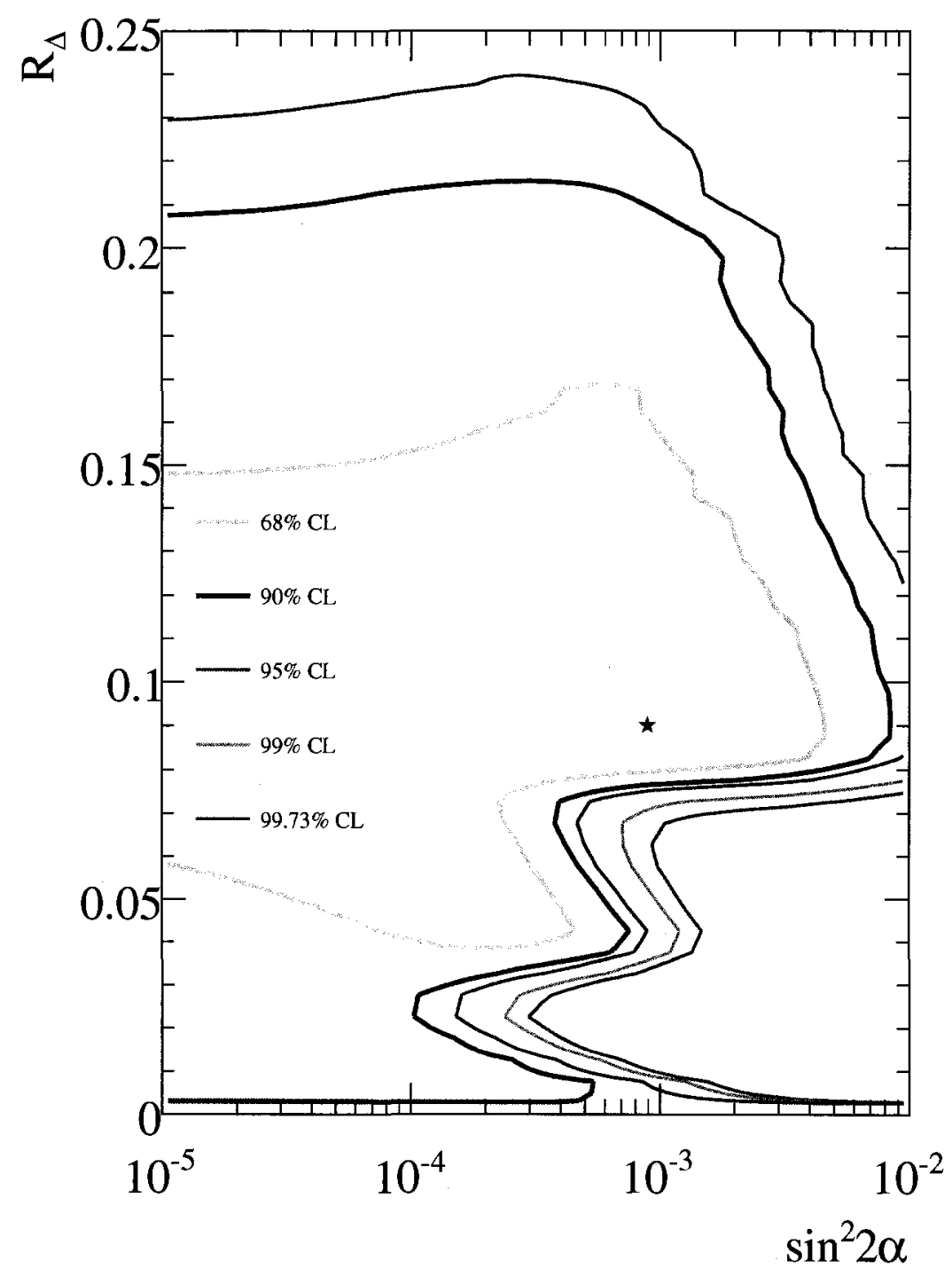

Figure 8.19: Global $\chi^{2}$ confidence level contours of the sterile neutrino parameter space. This analysis includes SNO-I summed kinetic energy spectra (day and night); SNO-II CC kinetic energy spectra, NC and ES fluxes (day and night), SK zenith binned kinetic energy spectra; and the rate measurements from the Homestake, Gallex/GNO and SAGE. The best-fit point is at $\sin ^{2} 2 \alpha=8.9 \times 10^{-4}$, $R_{\Delta}=0.09$ and $\phi_{B_{\mathrm{B}}}=5.32 \times 10^{6} \mathrm{~cm}^{-2} \mathrm{~s}^{-1}$ with $\chi_{\min }^{2}=118.76 / 116(d o f)$. The LMA parameters used are $\Delta m_{21}^{2}=7.1 \times 10^{-5} \mathrm{eV}^{2}$ and $\tan ^{2} \theta=0.40$. 

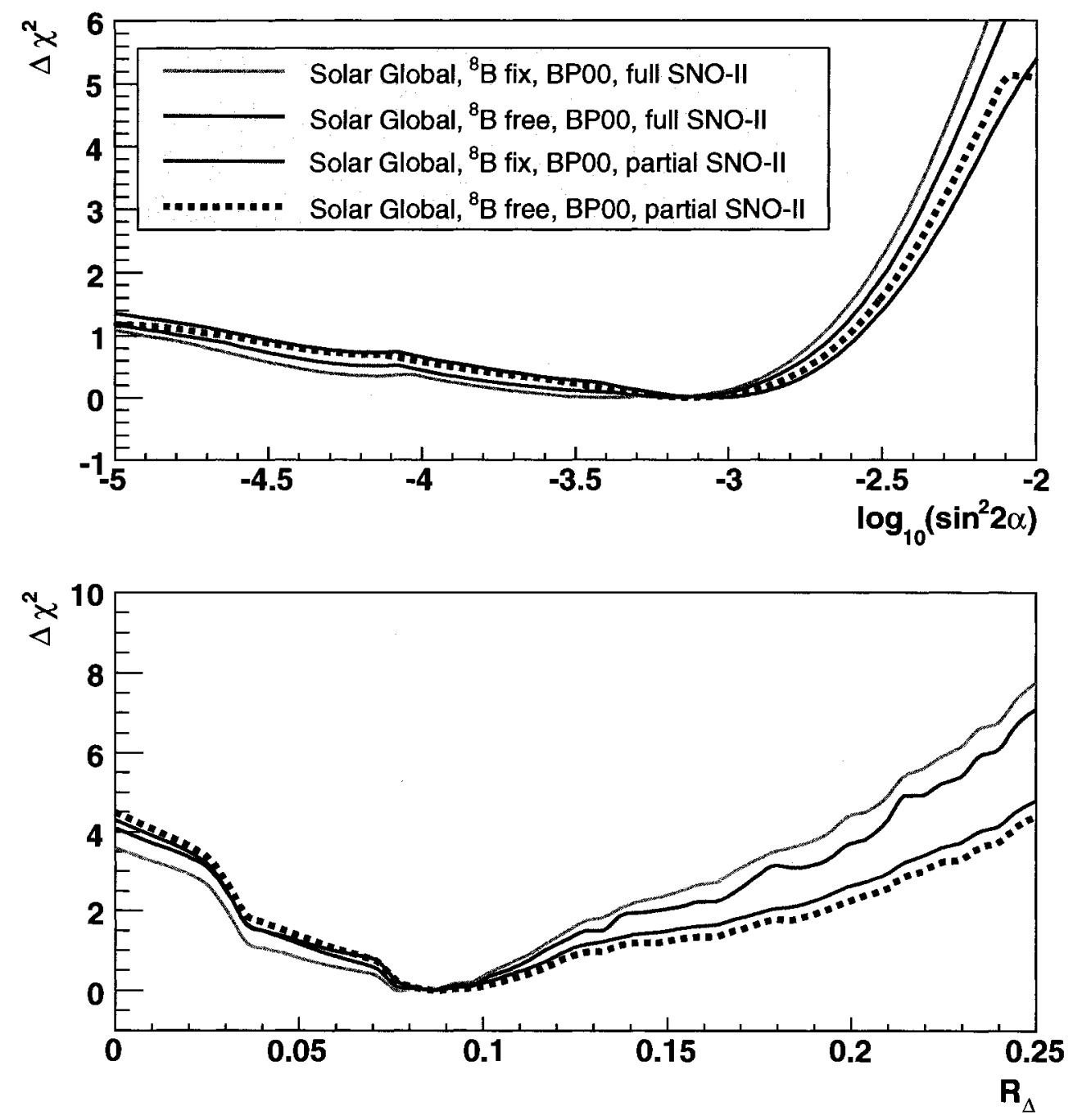

Figure 8.20: One dimensional projections of the $\Delta \chi^{2}$ function on $\sin ^{2} 2 \alpha$ and $R_{\Delta}$ axis for the global solar analyses. The red and blue dotted lines correspond to the global analyses with the partial data sample from SNO-II, while the green and blue lines are for the analysis with the full SNO-II data sample. View this figure in colour. 
neutrino flux fixed and free analyses, respectively. The results are summarized in Table 8.4.

Table 8.4: Global solar best-fit parameters after the SNO-III phase. The ${ }^{8} \mathrm{~B}$ neutrino fluxes are expressed in units of $10^{6} \mathrm{~cm}^{-2} \mathrm{~s}^{-1}$. All fits are performed with $\Delta m_{21}^{2}=7.1 \times 10^{-5} \mathrm{eV}^{2}$ and $\tan ^{2} \theta=0.40$.

\begin{tabular}{lll}
\hline \hline Parameter & SSM $^{8}$ B flux & Free ${ }^{8}$ B flux \\
\hline \multicolumn{3}{c}{ After SNO-III } \\
\hline $\sin ^{2} 2 \alpha$ & $<7.7 \times 10^{-3}$ at $90 \%$ CL & $<9.9 \times 10^{-3}$ at $90 \%$ CL \\
$R_{\Delta}$ & $0.10_{-0.02}^{+0.04}$ & $0.11_{-0.03}^{+0.04}$ \\
$\phi_{8}$ & 5.69 & 5.66 \\
\hline \hline
\end{tabular}

The confidence level contours in the $\sin ^{2} 2 \alpha-R_{\Delta}$ plane are depicted in Figures 8.21 and 8.22 , with the fixed and fitted ${ }^{8} \mathrm{~B}$ neutrino fluxes, respectively. The preferred regions have higher sterile neutrino mixing parameters than in analyses performed before the SNO-III phase. The most significant improvement is observed in $68 \%$ CL contour, that is both reduced in size and for the first time completely contained in the scanned parameter region. The standard $1 \sigma$ errors on the mixing parameters are extracted from the one dimensional projections of the global $\Delta \chi^{2}$ functions depicted in Figure 8.23.

The weakly mixed sterile neutrino prediction for the CC kinetic energy spectra for SNO-II at the best-fit point for the ${ }^{8} \mathrm{~B}$ neutrino flux free analysis is plotted in Figure 8.24 with the data and also with the best fit LMA prediction from global solar+KamLAND analyses after the SNO-III phase (c.f. Table 7.14). Both the two-neutrino and sterile neutrino interpretations described well the SNO data. The $p$-value for the two-neutrino model is 0.01 , while $p$-value for the weakly mixed sterile neutrino model is 0.06 , suggesting a slightly better fit to the data. The 


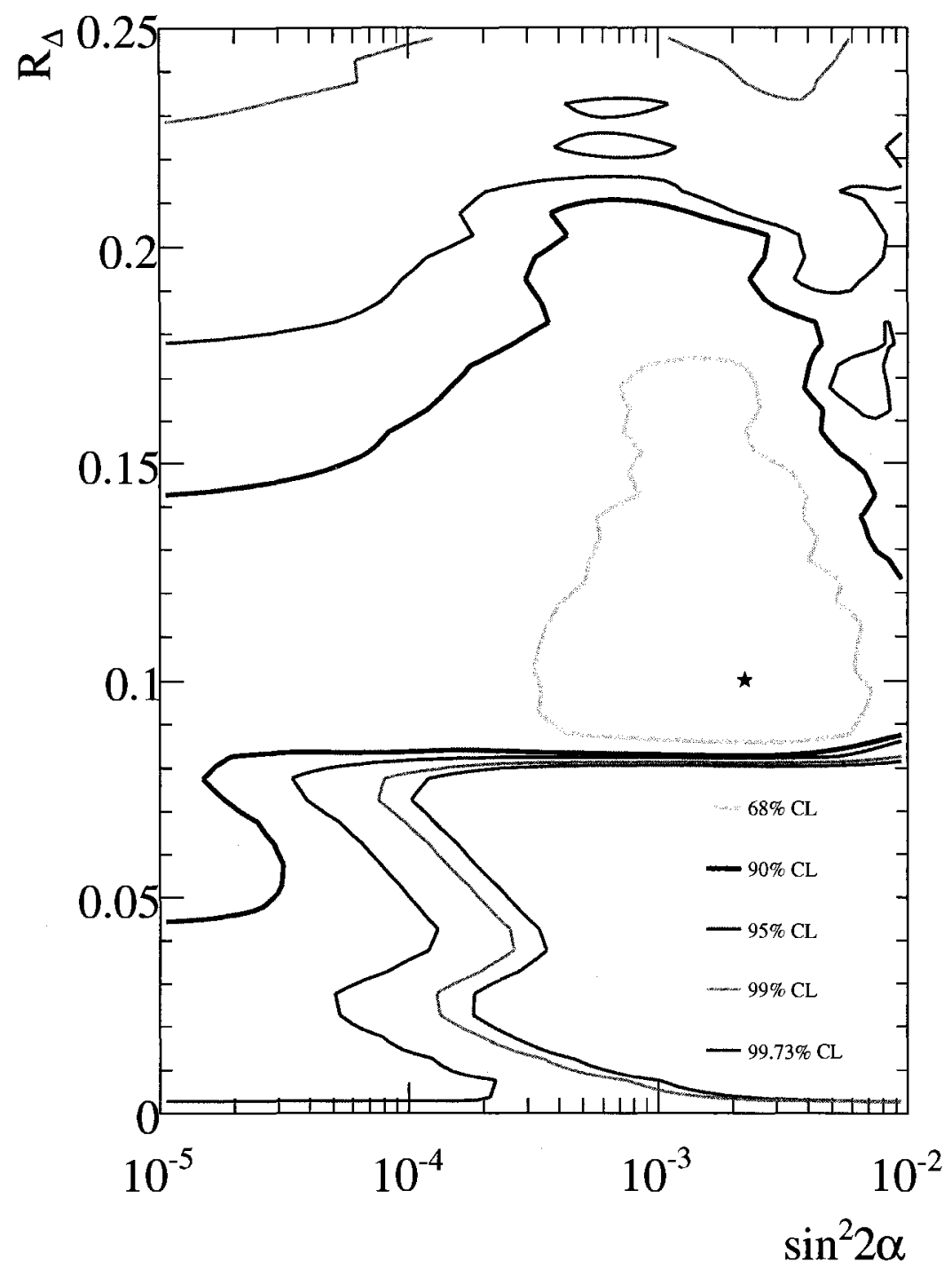

Figure 8.21: Global $\chi^{2}$ confidence level contours of the sterile neutrino parameter space. This analysis includes SNO-I summed kinetic energy spectra (day and night); SNO-II CC kinetic energy spectra, NC and ES fluxes (day and night); SNO-III CC, ES and NC fluxes; SK zenith binned kinetic energy spectra; and the rate measurements from the Homestake, Gallex/GNO, SAGE and Borexino. The best fit point is at $\sin ^{2} 2 \alpha=2.2 \times 10^{-3}$ and $R_{\Delta}=0.10$ with $\chi_{\min }^{2}=131.12 / 121$ (dof). The ${ }^{8} \mathrm{~B}$ neutrino flux was fixed to $5.69 \times 10^{6} \mathrm{~cm}^{-2} \mathrm{~s}^{-1}$. The LMA parameters used are $\Delta m_{21}^{2}=7.1 \times 10^{-5} \mathrm{eV}^{2}$ and $\tan ^{2} \theta=0.40$. 


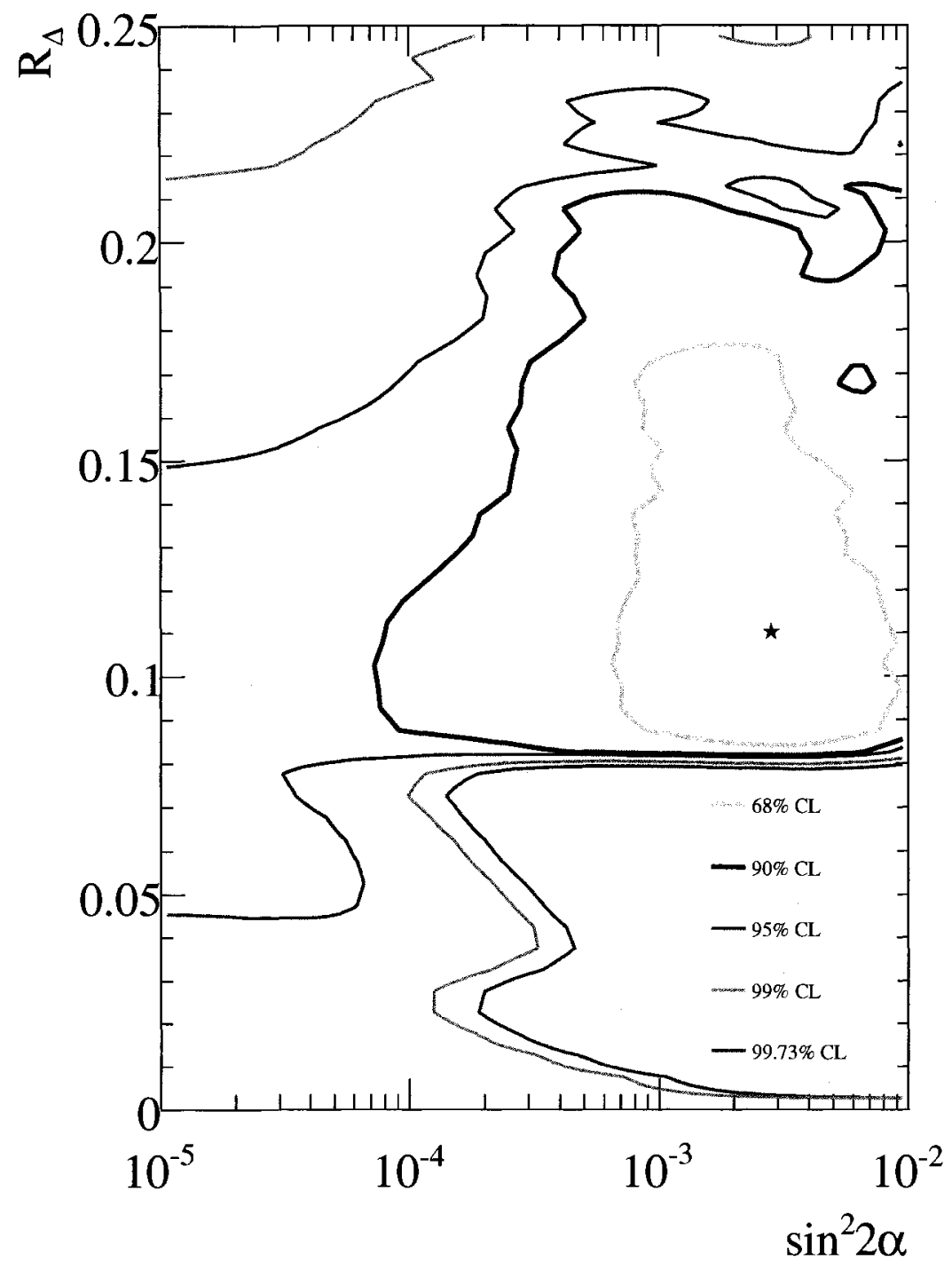

Figure 8.22: Global $\chi^{2}$ confidence level contours of the sterile neutrino parameter space. This analysis includes SNO-I summed kinetic energy spectra (day and night); SNO-II CC kinetic energy spectra, NC and ES fluxes (day and night), SNO-III CC, ES and NC fluxes; SK zenith binned kinetic energy spectra; and the rate measurements from the Homestake, Gallex/GNO, SAGE and Borexino. The best-fit point is at $\sin ^{2} 2 \alpha=2.8 \times 10^{-3}, R_{\Delta}=0.11$ and $\phi_{8_{\mathrm{B}}}=5.66 \times 10^{6} \mathrm{~cm}^{-2} \mathrm{~s}^{-1}$ with $\chi_{\min }^{2}=132.03 / 120($ dof $)$. The LMA parameters used are $\Delta m_{21}^{2}=7.1 \times 10^{-5}$ $\mathrm{eV}^{2}$ and $\tan ^{2} \theta=0.40$. 

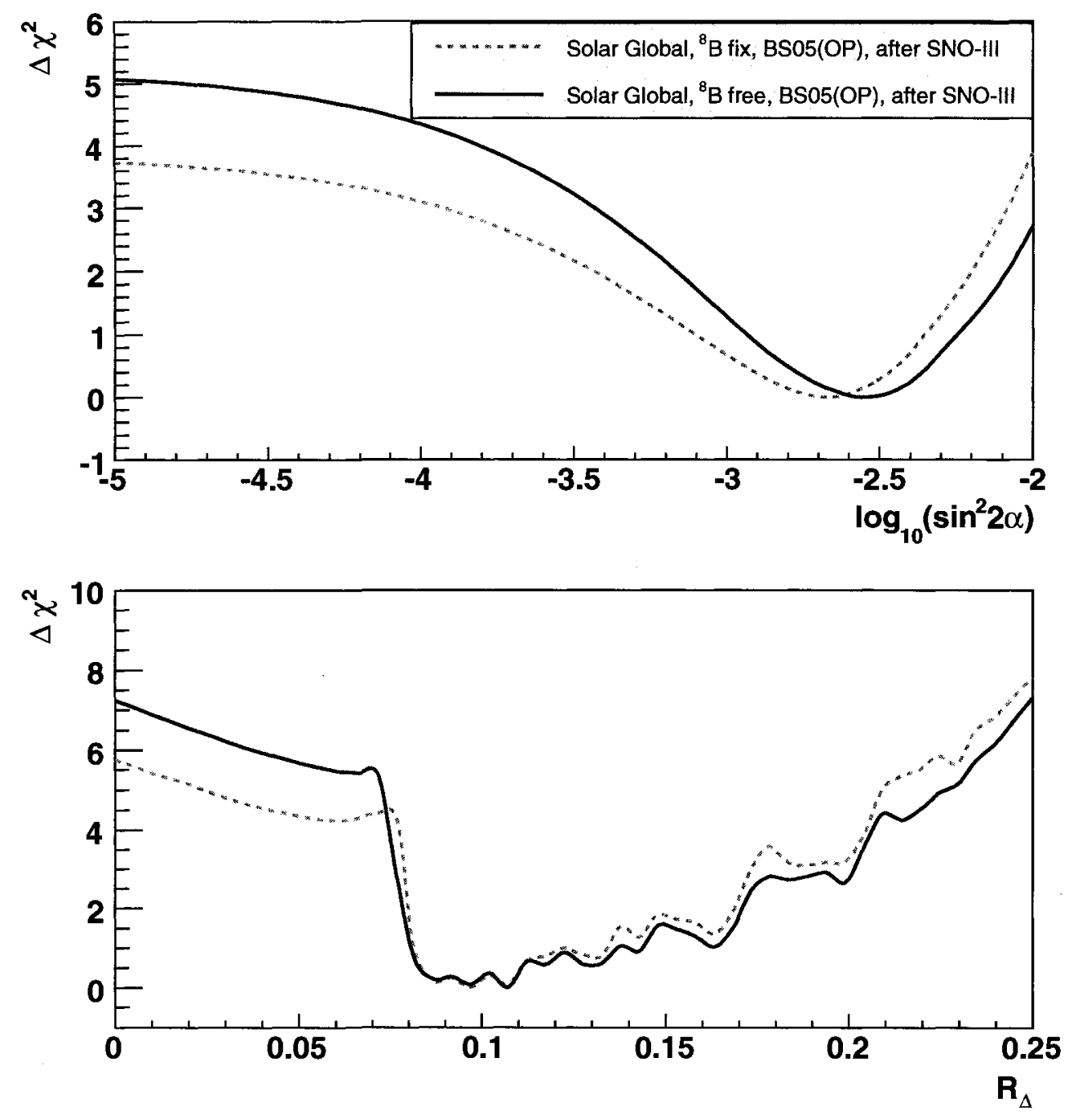

Figure 8.23: One dimensional projections of the $\Delta \chi^{2}$ function on $\sin ^{2} 2 \alpha$ and $R_{\Delta}$ axis for the global solar analyses after SNO-III. The green dotted and blue solid lines correspond to the global analyses with the fixed and free ${ }^{8} \mathrm{~B}$ neutrino flux, respectively. 
expected rate for the sterile model at Homestake is derived to be $2.75 \mathrm{SNU}$ for the SSM value of the ${ }^{8} \mathrm{~B}$, and $2.74 \mathrm{SNU}$ from the free ${ }^{8} \mathrm{~B}$ analysis. Both results agree with the experimental measurement of $(2.56 \pm 0.23) \mathrm{SNU}$, within the $1 \sigma$ error.

\subsection{Summary}

Under the assumption of the two-active and a single weakly mixed sterile neutrino model, the transition probabilities are computed for solar neutrinos and then used to infer the expected neutrino yields for a number of solar neutrino experiments. These yields are used for the extraction of the sterile neutrino mixing parameters.

The data samples from the three SNO phases alone are not sufficient for constraining the sterile mixing angle parameter $\sin ^{2} 2 \alpha$ and only an upper limit at $90 \% \mathrm{CL}$ is placed on the ratio $R_{\Delta}$. The result from the global solar analysis with the latest SNO-III data places, for the first time, limits on the allowed region in the $\sin ^{2} 2 \alpha-R_{\Delta}$ plane. The extracted parameters from this analysis are $\sin ^{2} 2 \alpha<9.9 \times 10^{-3}$ at $90 \% \mathrm{CL}$ and $R_{\Delta}=0.11_{-0.03}^{+0.04}$. Unfortunately, the actual limit based on the analysis performed here does not constrain the weakly mixed sterile neutrino mixing parameters very well. Hence the solar neutrino data cannot confirm the presence of sterile neutrinos at the $5 \sigma$ level. Our analysis suggested that the sterile model is a slightly better interpretation than the default twoneutrino oscillation mechanism, but the data is pretty much consistent with the LMA solutions. Nonetheless both two-neutrino and sterile neutrino models fits well the solar neutrino data.

Because the electron neutrino survival probabilities are affected by a sterile 


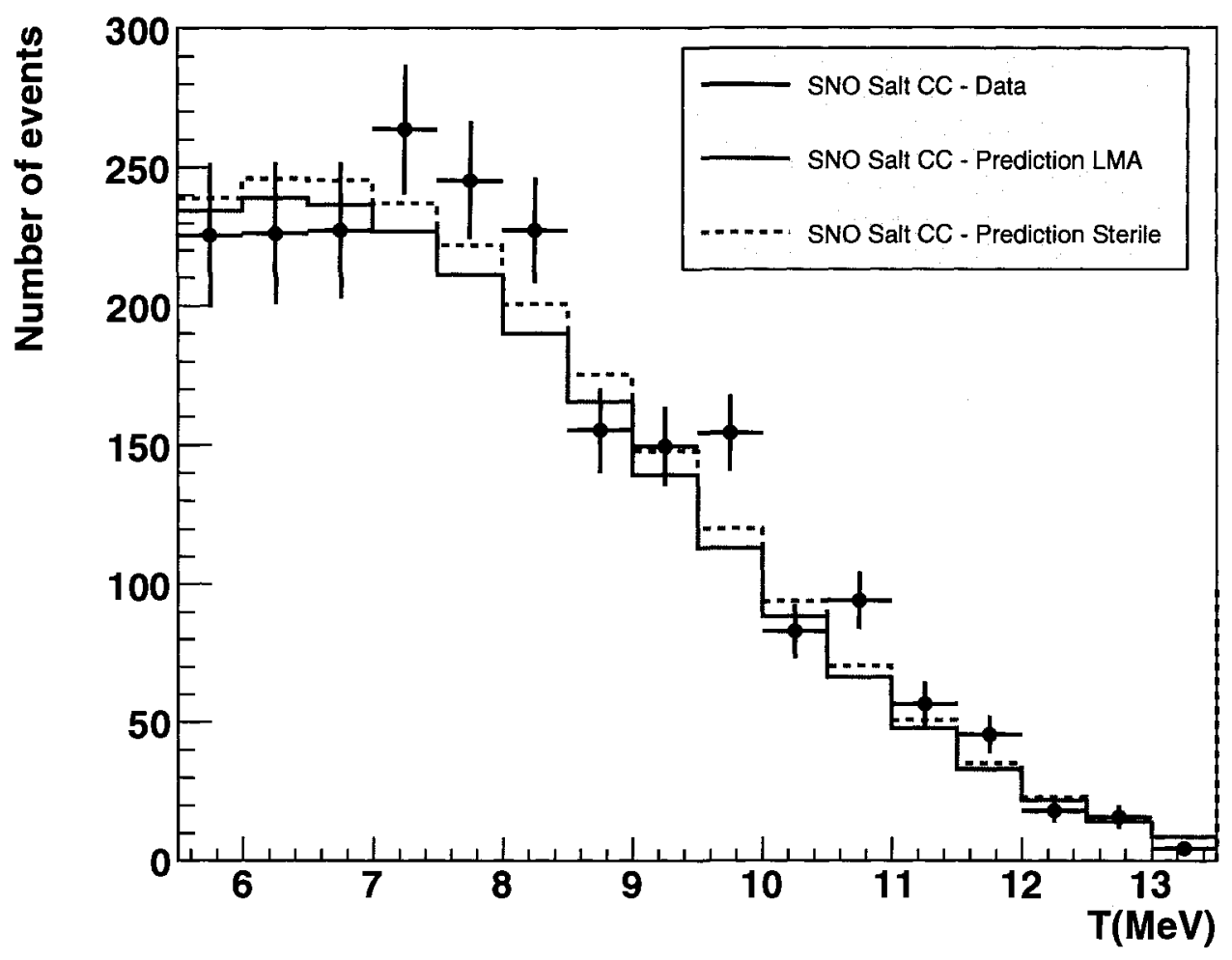

Figure 8.24: The kinetic energy CC spectra from the SNO-II phase averaged over day and night. The data are depicted as dots with error bars. The newest LMA prediction for the two-neutrino model is the solid line; and the latest weakly mixed sterile neutrino prediction is the dotted line. 
neutrino mainly at lower energies, the improvements in the analyses are expected with the inclusion of data from the Low Energy Threshold Analyses (LETA) from SNO-I and SNO-II. The $\mathrm{D}_{2} \mathrm{O}$ and salt data samples from LETA will have a kinetic energy threshold at $3.5 \mathrm{MeV}$, where the survival probability flattens or has a dip due to the sterile neutrino mixing. The LETA analysis is also planning to add the constraint from the SNO-III phase with NCD measurement of the NC flux. The latest rate results of the ${ }^{8} \mathrm{~B}$ neutrinos above $2.8 \mathrm{MeV}$ and the $0.862 \mathrm{MeV}^{7} \mathrm{Be}$ neutrinos by the Borexino experiment [95] will also further constrain the weakly mixed sterile neutrino parameter space. The SNO collaboration is planning to publish the LETA analysis in early January 2009. The sterile analysis presented here will then be updated by the author. It is expected to improve significantly the limit on the sterile mixing parameters as it will be discussed in Chapter 9 . 


\section{Chapter 9}

\section{Future Prospects}

In this chapter, possible future improvements to the analyses presented in this dissertation are discussed.

\subsection{Low Energy Solar Neutrino Data}

\subsubsection{LETA Expectation}

The most significant input that will improve the constraints on the solar neutrino mixing parameters in the context of the matter induced two-active flavour oscillation scenario is the upcoming Low Energy Threshold Analysis (LETA) by the SNO Collaboration. LETA expects to produce CC and ES spectra for day and night at a kinetic energy threshold of $3.5 \mathrm{MeV}$, together with a day and night $\mathrm{NC}$ fluxes for both the $\mathrm{D}_{2} \mathrm{O}$ (SNO-I) and salt (SNO-II) phases. LETA will also incorporate the NC flux extracted during the NCD (SNO-III) phase. The error on $\tan ^{2} \theta_{12}$ and $\Delta m_{21}^{2}$ is expected to be reduced by about $5 \%$ and $10 \%$, respectively. 


\subsubsection{Sterile Neutrinos}

As presented in Chapter 8, a weakly mixed sterile neutrino mainly affects solar neutrino transition probabilities at low and intermediate neutrino energies. Thus, it is crucial to test the measurements from experiments that are sensitive in the low neutrino energy regime. At present, there is a single measurement of this type that comes from the Borexino experiment [47]. The latest measurement of the ${ }^{8} \mathrm{~B}$ neutrino rate above the energy threshold of $2.8 \mathrm{MeV}$ and also the $0.862 \mathrm{MeV}$ ${ }^{7}$ Be neutrino rate by Borexino [95] must be included in future tests of the weakly sterile neutrino model. It is expected that this measurement should significantly constrain a region in the $\sin ^{2} 2 \alpha-R_{\Delta}$ plane that correspond to low values of the ratio $R_{\Delta}$.

The other major improvement is expected from a forthcoming LETA analysis at SNO with the data sample extracted at the electron kinetic energy threshold of $3.5 \mathrm{MeV}$ for both SNO-I and SNO-II data samples. The LETA data set will help to further constrain the intermediate values for both sterile neutrino mixing parameters.

The analyses presented in this dissertation are performed under the assumption of particular mixing parameters for the two active neutrino flavours $\tan ^{2} \theta=0.40$ and $\Delta m^{2}=7.1 \times 10^{-6} \mathrm{eV}^{2}$. It is possible to re-scale the parameter $\Delta m^{2}$ without recomputing the transition probabilities due to the form of the Hamiltonian, where each squared-mass difference enters the calculations as a ratio with respect to the neutrino energy. Hence, changing the energies with the fixed squared-mass difference is equivalent to changing the squared-mass difference at a particular neutrino energy. However, this is not true for the mixing angle. It is desirable to scan the LMA region for the mixing angle $\theta$ and recompute the neutrino transition prob- 
abilities. One can then perform an analysis of the global solar neutrino data and extract simultaneously all four independent neutrino mixing parameters: $\Delta m^{2}$, $\tan ^{2} \theta, R_{\Delta}$ and $\sin ^{2} 2 \alpha$, as well as the ${ }^{8} \mathrm{~B}$ neutrino flux. This proposal demands an excessive amount of computing time. The CPU intense calculation has been the only limitation for not completing this analysis. Our study suggests that the limit on $\sin ^{2} 2 \alpha$ and $R_{\Delta}$ would not change significantly for different values of $\tan ^{2} \theta$ and $\Delta m^{2}$.

\subsubsection{Three Active Neutrinos}

The default physics interpretation at SNO uses the two-neutrino mixing model in order to extract the neutrino mixing parameters. The two parameters, as discussed in Chapters 2 and 7 , are $\Delta m^{2}$ and $\tan ^{2} \theta$. Knowing that there are three active neutrino flavours, the proper method is to use the three-neutrino mixing model with the $3 \times 3$ unitary mixing matrix given by Equation (2.12). It is an experimental fact that the mixing angle of the third mass-eigenstate $\nu_{3}$ in the electron neutrino flavour state $\nu_{e}$ is small $\left(\sin ^{2} 2 \theta_{13}<0.17\right.$, at 90\% CL [29]), and that $\Delta m_{31}^{2}$, which satisfies the equality:

$$
\left|\Delta m_{31}^{2}\right|=\left|m_{3}^{2}-m_{1}^{2}\right|=\left|\Delta m_{32}^{2}+\Delta m_{21}^{2}\right|
$$

is much greater than $m_{21}^{2}$. The latest results from the MINOS experiment is $\left|\Delta m^{2}{ }_{32}\right|=(2.43 \pm 0.13) \times 10^{-3} \mathrm{eV}^{2}$ at $68 \%$ CL [96]. These two experimental results allow for the LMA solution in two-neutrino mixing framework to describe solar neutrino data with very good accuracy.

However, by using the three-neutrino mixing hypothesis, the solar neutrino 
data could be used to constrain the mixing parameter $\theta_{13}$, and perhaps allowing for a better limit than the one that is presently reported of $\sin ^{2} 2 \theta_{13}<$ 0.17 , at $90 \% \mathrm{CL}$ [29]. In general, the effect of the third neutrino mixing is to lower the electron neutrino survival probabilities, as depicted in Figure 9.1. There has been an extensive number of global solar analyses in the three-neutrino framework, that is reported in the literature (see for example References [97-99]). The analyses of this type used the analytical approximative formulae up to the second-order effects due to the angle $\theta_{13}$. The reason for this approximation is the fact that numerical propagation of the three-neutrino system requires an extensive amount of computing time. In Appendix A, the numerical method is presented for the upcoming three-neutrino mixing analysis. It is expected that a global solar threeactive neutrino analysis will set a limit of about $\sin ^{2} 2 \theta_{13}<0.14$ at the $90 \%$ CL. For the combined global solar+KamLAND analysis the expected limit is about $\sin ^{2} 2 \theta_{13}<0.12$ at the $90 \% \mathrm{CL}$. These two limits are estimated by using the results in Reference [99]. The SNO Collaboration is presently preparing a full numerical calculation of the survival probability for a simultaneous fit of $\theta_{12}, \Delta m_{21}^{2}$ and $\theta_{13}$.

\subsection{Maximum Likelihood Method}

In the extraction of the solar neutrino mixing parameters, the covariance $\chi^{2}$ method is the default tool used by SNO. The $\chi^{2}$ method assumes Gaussian distribution for errors on the estimators and thus approximates the classical frequentist confidence intervals. It is a good method for the binned spectral data, only if there are enough expected events in each spectral bin. In the case where the number of 


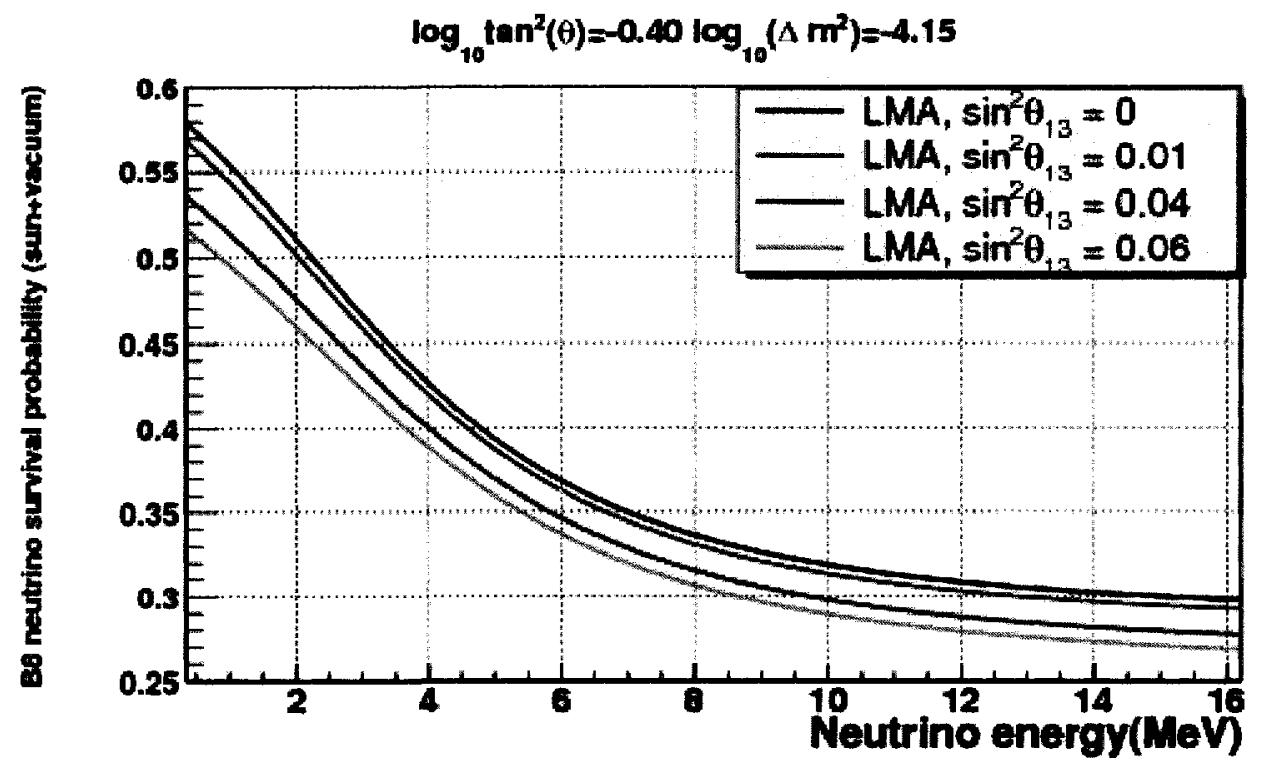

Figure 9.1: ${ }^{8} \mathrm{~B}$ electron neutrino survival probabilities for the Sun and vacuum for $\sin ^{2} \theta_{13}=0$ (black); $\sin ^{2} \theta_{13}=0.01$ (red); $\sin ^{2} \theta_{13}=0.04$ (blue); and $\sin ^{2} \theta_{13}=0.06$ (cian) for $\Delta m^{2}=7.1 \times 10^{-5} \mathrm{eV}^{2}, \tan ^{2} \theta=0.40$ and $\Delta m_{31}^{2}=2.7 \times 10^{-3} \mathrm{eV}^{2}$. View this figure in colour. 
events in a given bin is small, the $\chi^{2}$ method breaks down.

The Extended Maximum Likelihood (EML) method is more robust and does not rely on the Gaussian distribution of the errors on the estimators. It has been shown (see, for example Reference [83]) that if there is an efficient estimator for a given problem, the EML method will find it. The statistical EML function $L$ is defined as:

$$
-2 \ln L=2 Y_{\text {tot }}^{\text {th }}-2 \sum_{i=1}^{N} Y_{i}^{\text {exp }} \ln Y_{i}^{\text {th }},
$$

where $Y_{i}^{\text {th }}$ is the theoretical expectation (e.g. the model prediction for yields from the neutrino interactions at a given detector) over a set of $N$ observables $Y_{i}$. The EML method also allows for an un-binned analysis of spectral data, which can be applied to the SNO data. Furthermore there is more information contained in the signal that has not been used in the default oscillation analysis. In particular, the information about the angle between the reconstructed event direction and the direction of the Sun as well as isotropy and radial distributions of the signal. Due to the limited statistics in the SNO data samples, an additional binning with respect to those observables will push the $\chi^{2}$ method to its limit and would force the usage of the EML method.

The inclusion of correlated sources of errors in the likelihood function is a nontrivial task. The so-called pull method [101] has been applied in our EML analyses, which is described in Appendix B. We carried a number of tests with the EML function using spectral data from SNO to insure that the results obtained are in agreement with the default $\chi^{2}$ analyses. The best-fit points from the $\chi^{2}$ and EML methods for the SNO-I summed kinetic energy spectra $(\mathrm{CC}+\mathrm{ES}+\mathrm{NC}+$ background $)$ are found to be identical, with a minimal difference in the projected $1 \sigma$ errors. 
The EML and $\chi^{2}$ results are summarized in Table 9.1. The confidence regions from the two methods are depicted in Figures 9.2 and Figure 9.3 for both the EML and $\chi^{2}$, respectively.

Table 9.1: SNO-I neutrino oscillation best-fit parameters.

\begin{tabular}{lccc}
\hline \hline Analysis & $\Delta m^{2}\left(10^{-5} \mathrm{eV}^{2}\right)$ & $\tan ^{2} \theta$ & Total ${ }^{8} \mathrm{~B}$ flux $\left(10^{6} \mathrm{~cm}^{-2} \mathrm{~s}^{-1}\right)$ \\
\hline EML & $5.01_{-1.92}^{+6.74}$ & $0.45_{-0.08}^{+0.13}$ & 5.20 \\
$\chi^{2}$ & $5.01_{-1.91}^{+6.63}$ & $0.45_{-0.08}^{+0.12}$ & 5.21 \\
\hline \hline
\end{tabular}

However, the statistical correlations between SNO fluxes cannot be included directly in the likelihood function. The EML method was nevertheless used to extract mixing parameters for the SNO-only (SNO-I, SNO-II and SNO-III) analysis. The result yields: $\Delta m^{2}=4.47_{-1.19}^{+2.72} \times 10^{-5} \mathrm{eV}^{2}$ and $\tan ^{2} \theta=0.447_{-0.078}^{+0.068}$. The mass-squared difference has been shifted towards lower values, while the $1 \sigma$ errors on the mixing angle has increased by a small amount when compared to the results presented in Chapter 7. The allowed regions from the EML method with the SNO-only data are depicted in Figure 9.4. These regions are slightly larger than, but yet consistent, with the regions obtained by the default $\chi^{2}$ method due to the non-inclusion of the statistical correlations. The EML calculation is an excellent check that the $\chi^{2}$ method is valid even for a large number of observables. The EML is also a robust way to investigate the pull of many experimental systematic uncertainties. The inclusion of the statistical correlation into the EML function is possible by adding a $\chi^{2}$ terms for the statistically correlated binned data and total integrated fluxes.

This could represent an improvement for the extraction of the neutrino mixing 
parameters by using the four relevant observables at SNO; namely, energy, position, direction and isotropy. This would lead to slightly better constraints on the allowed regions in $\Delta m^{2}-\tan ^{2} \theta$ plane, while insuring reliable confidence intervals.

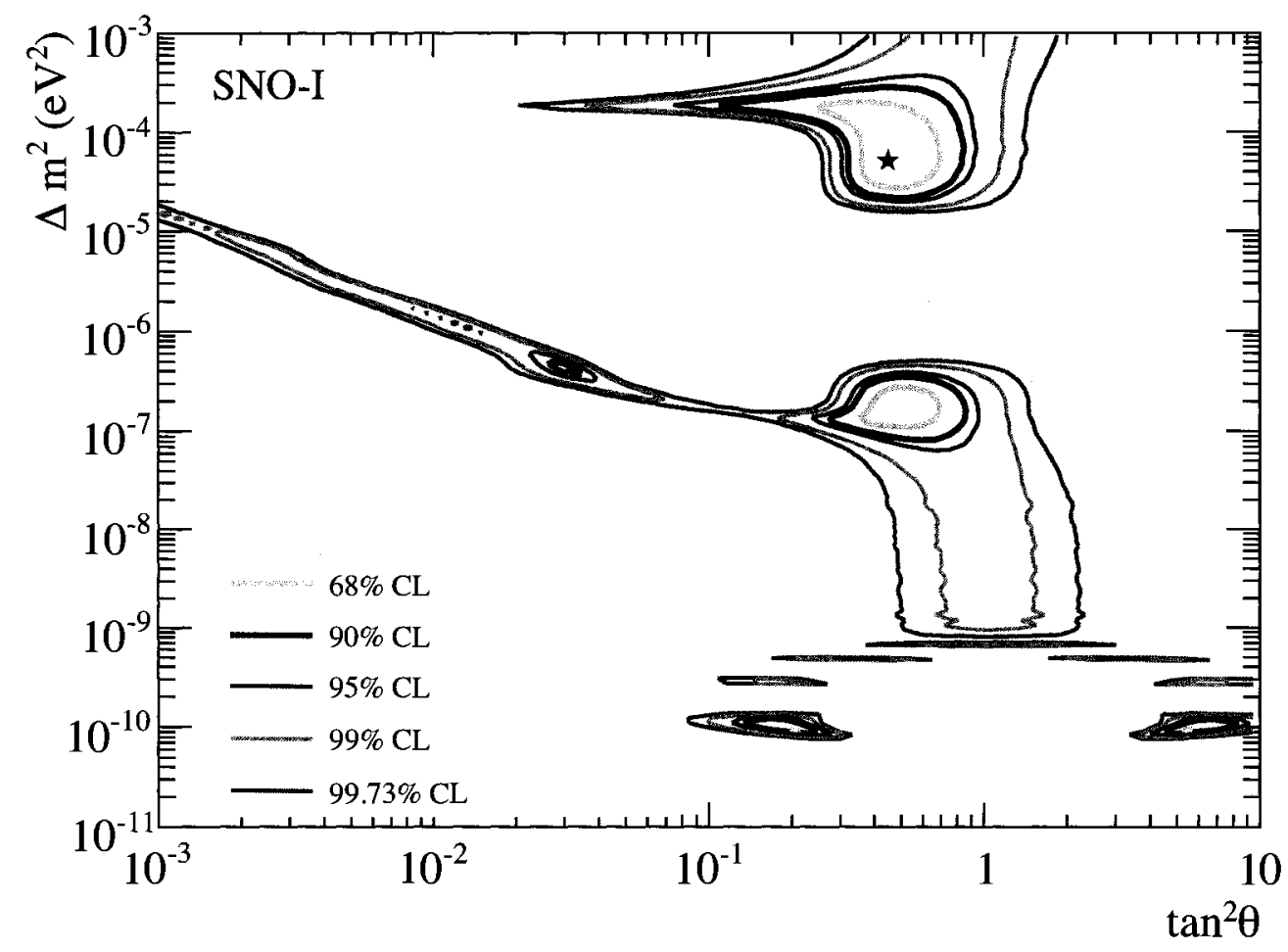

Figure 9.2: SNO-I only neutrino oscillation contours from the EML analysis. This analysis includes summed kinetic energy spectra $\mathrm{CC}+\mathrm{NC}+\mathrm{ES}+$ background from SNO-I (day and night). The best fit point is at: $\Delta m^{2}=5.01 \times 10^{-5} \mathrm{eV}^{2}$, $\tan ^{2} \theta=0.447, \phi_{8_{\mathrm{B}}}=5.20 \times 10^{6} \mathrm{~cm}^{-2} \mathrm{~s}^{-1}$. The hep neutrino flux was fixed at $7.93 \times 10^{3} \mathrm{~cm}^{-2} \mathrm{~s}^{-1}$. 


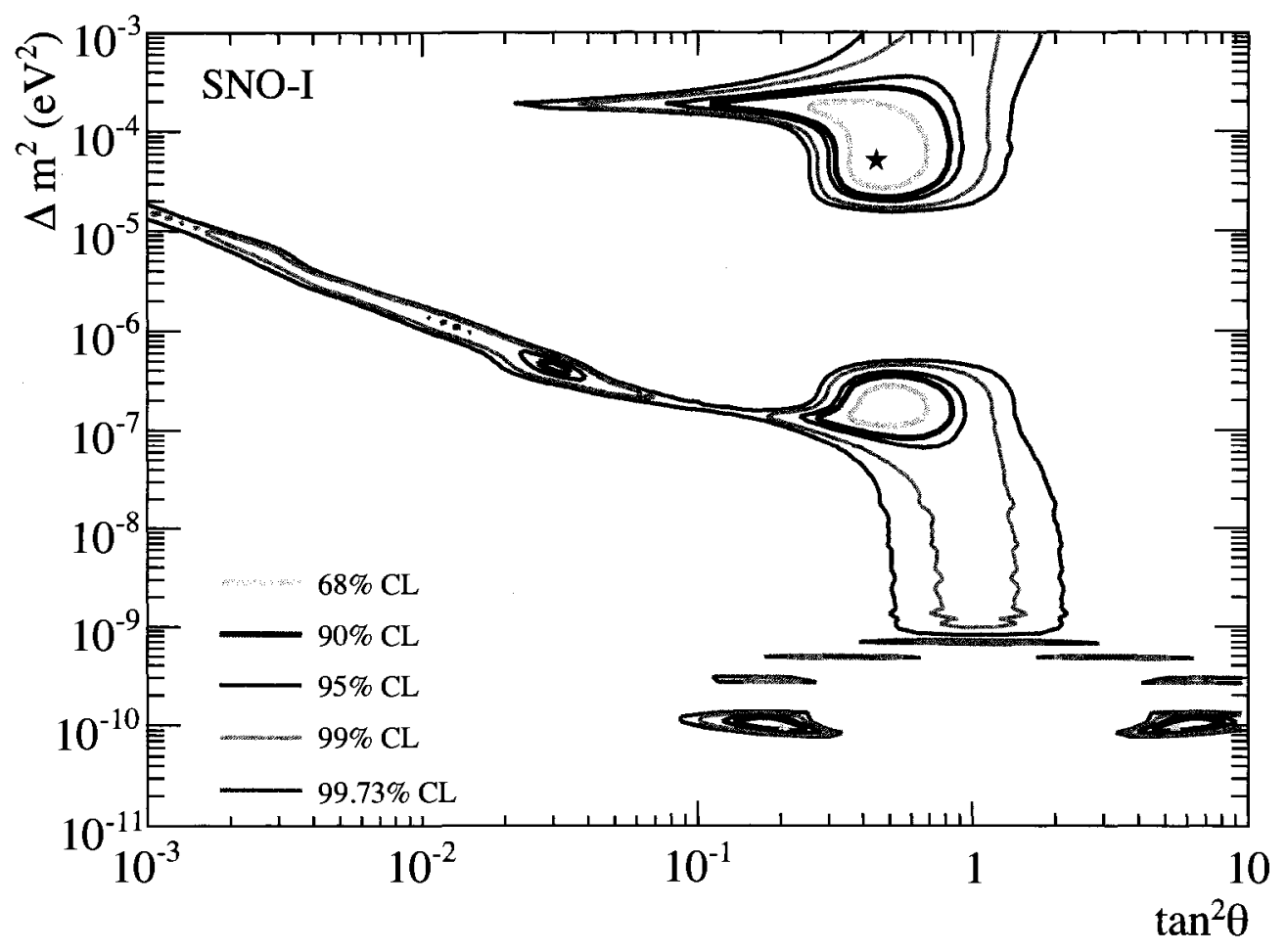

Figure 9.3: SNO-I only neutrino oscillation contours from the $\chi^{2}$ analysis. This analysis includes summed kinetic energy spectra $\mathrm{CC}+\mathrm{NC}+\mathrm{ES}+$ background from SNO-I. The best fit point is at: $\Delta m^{2}=5.01 \times 10^{-5} \mathrm{eV}^{2}, \tan ^{2} \theta=0.447, \phi_{8_{\mathrm{B}}}=$ $5.21 \times 10^{6} \mathrm{~cm}^{-2} \mathrm{~s}^{-1}$. The hep neutrino flux was fixed at $7.93 \times 10^{3} \mathrm{~cm}^{-2} \mathrm{~s}^{-1}$. 


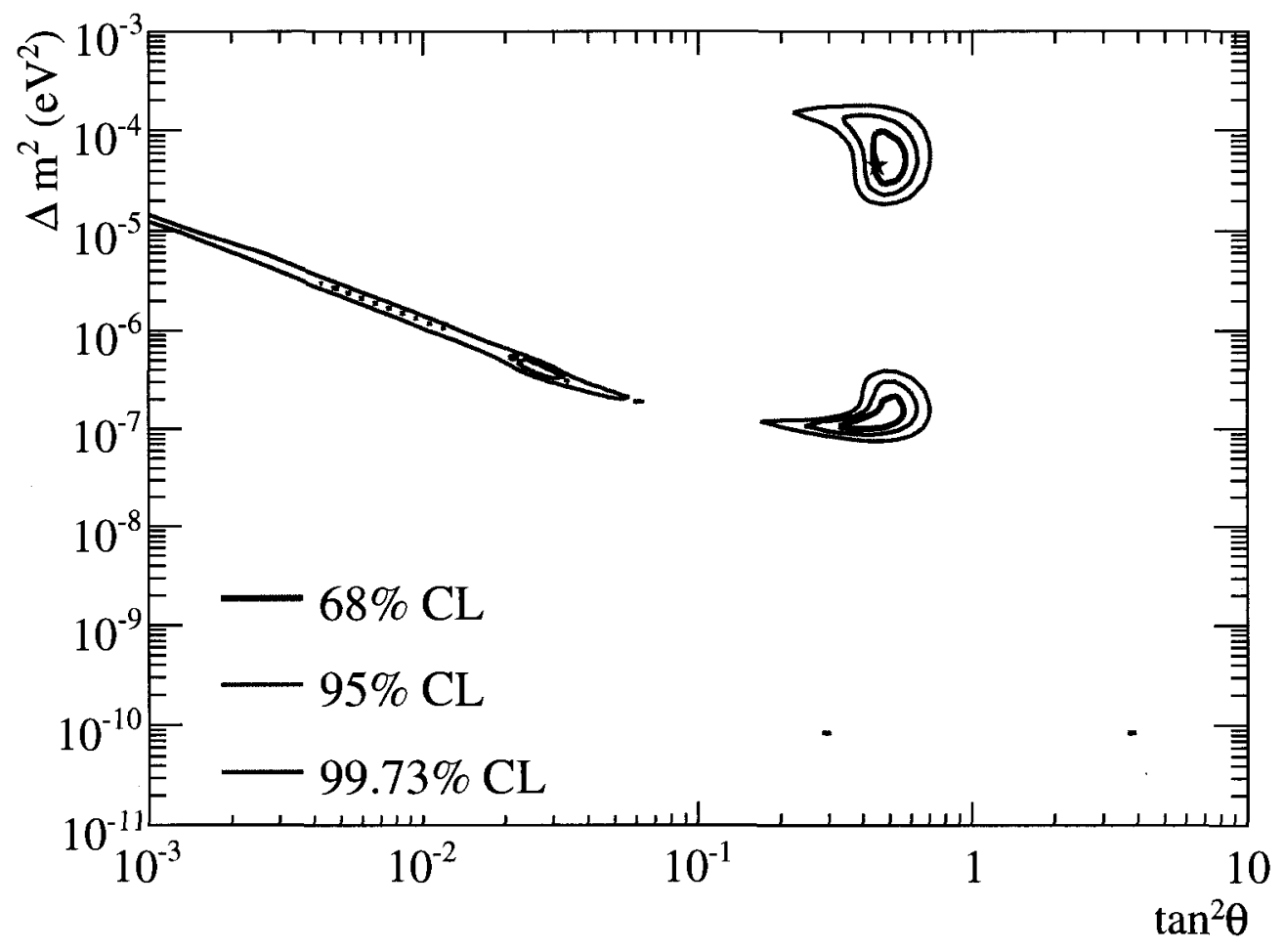

Figure 9.4: SNO only neutrino oscillation contours from the EML analysis. This analysis includes summed kinetic energy spectra from SNO-I (day and night); NC, ES fluxes, and CC kinetic energy spectra from SNO-II (day and night); and CC, $\mathrm{NC}$ and ES fluxes from SNO-III. The best fit point is at: $\Delta m^{2}=4.47 \times 10^{-5} \mathrm{eV}^{2}$, $\tan ^{2} \theta=0.447, \phi_{8 \mathrm{~B}}=5.12 \times 10^{6} \mathrm{~cm}^{-2} \mathrm{~s}^{-1}$. The hep neutrino flux was fixed at $7.93 \times 10^{3} \mathrm{~cm}^{-2} \mathrm{~s}^{-1}$. To be compared with Figure 7.3 . 


\subsection{Discussion}

Some of the possible improvements to the neutrino analyses presented in this dissertation have been discussed. A significant improvement regarding both active and sterile neutrino models is expected with the inclusion of new low energy threshold data from the SNO LETA analyses. The solar neutrino data can also be used to place better limits on the $\theta_{13}$ mixing angle. Further improvements in the solar neutrino analyses are expected from future low energy neutrino measurements; and by new analysis techniques. The EML method has been discussed in this chapter.

There are many unanswered questions regarding neutrinos, some of which have been mentioned in the introduction to this dissertation. Those questions are still intriguing the author, whose interest in neutrino physics does not end here. 


\section{Chapter 10}

\section{Conclusion}

In this dissertation, the interpretation of solar neutrino data in the frameworks of the two distinct neutrino mixing models has been presented.

The analyses are performed with data collected by the Sudbury Neutrino Observatory. By using heavy water as a target for the weak interactions of neutrinos, the SNO detector has a unique property to measure the total flux of all active neutrino flavour states $\left(\nu_{e}, \nu_{\mu}\right.$ and $\left.\nu_{\tau}\right)$ via the neutral-current interaction. The measurements on the total neutrino flux from the ${ }^{8} \mathrm{~B}$ decay in the Sun are in agreement with the most recent standard solar models. SNO also measures the flux of electron neutrino state through the charged-current interaction. The NC and CC flux measurements are consistent with the model predictions from the two-neutrino oscillation hypothesis, in particular with the LMA solution.

The results from SNO represent a joint effort of a number of analysts, which assure the best quality of the data samples, analyses techniques and robust physics interpretation. For the purpose of this dissertation, the analyses of the data samples with the associated systematic errors would not be possible without results 
obtained by other SNO collaborators. The original contributions of the author within the SNO Collaboration has been the work presented in this thesis.

First, the derivation of the precise livetime for the SNO-III phase of running has been presented. The determined livetime distribution in respect to 480 bins of zenith angle has been used to properly weight the electron neutrino survival probabilities, which are necessary in order to derive the expected yields from the neutrino interactions at the SNO detector during the NCD phase (SNO-III). The final livetimes for the day, night and total are:

$$
\begin{aligned}
& \tau_{\text {day }}=(176.59 \pm 0.07) \text { days }, \\
& \tau_{\text {night }}=(208.58 \pm 0.07) \text { days, } \\
& \tau_{\text {total }}=(385.17 \pm 0.14) \text { days. }
\end{aligned}
$$

Next, the latest results of the solar neutrino mixing parameters from SNO [51], under assumption of the two-neutrino oscillation hypothesis have been reported. With the data samples from the SNO-I, SNO-II and SNO-III phases, the best-fit parameters from SNO-only analysis are:

$$
\begin{aligned}
& \Delta m^{2}=4.57_{-1.22}^{+2.30} \times 10^{-5} \mathrm{eV}^{2}, \\
& \tan ^{2} \theta=0.447_{-0.048}^{+0.045}
\end{aligned}
$$

with the fitted total active ${ }^{8} \mathrm{~B}$ solar neutrino flux of $\phi_{8_{\mathrm{B}}}=5.12 \times 10^{6} \mathrm{~cm}^{-2} \mathrm{~s}^{-1}$. The global solar analysis with the data from SNO, SK-I, Homestake, Gallex/GNO, SAGE and Borexino yields to mixing parameters:

$$
\begin{aligned}
& \Delta m^{2}=4.90_{-0.93}^{+1.64} \times 10^{-5} \mathrm{eV}^{2}, \\
& \theta=33.5_{-1.3}^{+1.3},
\end{aligned}
$$


with the fitted value of the ${ }^{8} \mathrm{~B}$ flux of $\phi_{8_{\mathrm{B}}}=5.21 \times 10^{6} \mathrm{~cm}^{-2} \mathrm{~s}^{-1}$. The final result on neutrino mixing parameters is obtained from the global analysis with the addition of the anti-neutrino results from the KamLAND reactor:

$$
\begin{aligned}
& \Delta m^{2}=7.59_{-0.21}^{+0.19} \times 10^{-5} \mathrm{eV}^{2}, \\
& \theta=34.4_{-1.2}^{+1.3} \text { degrees. }
\end{aligned}
$$

The corresponding flux of the ${ }^{8} \mathrm{~B}$ neutrinos is $\phi_{8_{\mathrm{B}}}=4.92 \times 10^{6} \mathrm{~cm}^{-2} \mathrm{~s}^{-1}$. This result further improves the SNO measurement on the solar neutrino mixing parameters by reducing the allowed region in the $\tan ^{2} \theta-\Delta m^{2}$ plane. This corresponds to a $45 \%$ improvement on the mixing angle over previously measured results [48]. It is to be noted that SNO with its ability to simultaneously measure total $\phi_{8_{\mathrm{B}}}$ and electron neutrino portion $\phi_{8 \mathrm{~B}, \nu_{\mathrm{e}}}$ of the ${ }^{8} \mathrm{~B}$ neutrino flux is the best solar neutrino experiment in defining the mixing angle; while KamLAND is the short-baseline experiment that is the best in measuring the squared-mass difference $\Delta m^{2}$.

In the weakly mixed sterile neutrino framework, the interpretation of the global solar neutrino data, which includes measurements from the SNO-I, SNO-II and SNO-III phases, SK-I, Homestake, Gallex/GNO, SAGE and Borexino leads to the sterile neutrino mixing parameters:

$$
\begin{aligned}
& R_{\Delta}=0.11_{-0.03}^{+0.04}, \\
& \sin ^{2} 2 \alpha<9.9 \times 10^{-3} \text { at } 90 \% \mathrm{CL},
\end{aligned}
$$

with the flux of the ${ }^{8} \mathrm{~B}$ of $\phi_{\mathrm{B}_{\mathrm{B}}}=5.66 \times 10^{6} \mathrm{~cm}^{-2} \mathrm{~s}^{-1}$ at $\Delta m^{2}=7.1 \times 10^{-5} \mathrm{eV}^{2}$ and $\tan ^{2} \theta=0.40$. For the first time, full constraint has been placed on the allowed region in the $\sin ^{2} 2 \alpha-R_{\Delta}$ plane. The further improvements to this analysis are 
expected with the inclusion of the forthcoming data from the upcoming SNO Low Energy Threshold Analysis (LETA), and also the latest measurements from the Borexino experiment. 


\section{Appendix A}

\section{The Three-Active Neutrinos}

The numerical integration method that can be applied in the propagation of the three-active neutrino states is described in this appendix.

The mixing matrix for the three active neutrino system given in Equation (2.12) can be also parameterized as [31]:

$$
U=U_{23} \Gamma U_{13} U_{12},
$$

where the matrices $U_{23}, \Gamma, U_{13}$ and $U_{12}$ are defined as:

$$
\begin{aligned}
\Gamma & =\left(\begin{array}{ccc}
1 & 0 & 0 \\
0 & e^{i \delta} & 0 \\
0 & 0 & e^{-i \delta}
\end{array}\right), \\
U_{23} & =\left(\begin{array}{ccc}
1 & 0 & 0 \\
0 & c_{23} & s_{23} \\
0 & -s_{23} & c_{23}
\end{array}\right),
\end{aligned}
$$




$$
U_{13}=\left(\begin{array}{ccc}
c_{13} & 0 & s_{13} \\
0 & 1 & 0 \\
-s_{13} & 0 & c_{13}
\end{array}\right),
$$

and

$$
U_{12}=\left(\begin{array}{ccc}
c_{12} & s_{12} & 0 \\
-s_{12} & c_{12} & 0 \\
0 & 0 & 1
\end{array}\right)
$$

where $c_{i j}$ and $s_{i j}(i, j=1,2,3)$ present the sine and cosine of the corresponding mixing angles $\theta_{i j}$, respectively.

This choice of parameterization allows for rotating away the mixing angle $\theta_{23}$ and the CP violating phase $\delta$ from the Hamiltonian $H$ that describes a propagation of the three neutrino system in matter $\left(H=H_{\mathrm{vac}}+H_{\mathrm{m}}\right.$, as given in Equations (2.44) and (2.45)). The rotated Hamiltonian $H^{\text {rot }}$ is obtained by performing this transformation:

$$
H^{\mathrm{rot}}=U_{13}^{\dagger} \Gamma^{\dagger} U_{23}^{\dagger} H U_{23} \Gamma U_{13}
$$

The elements $H_{i j}(i, j=1,2$ and 3$)$ of the resulting Hamiltonian are given as:

$$
\begin{aligned}
& H_{11}=\frac{1}{2 E}\left(\Delta m_{21}^{2} \sin ^{2} \theta_{12} \cos ^{2} \theta_{13}+\Delta m_{31}^{2} \sin ^{2} \theta_{13}^{2}\right)+V_{\mathrm{CC}}, \\
& H_{12}=H_{21}=\frac{1}{4 E}\left(\Delta m_{21}^{2} \sin 2 \theta_{12} \cos \theta_{13}\right), \\
& H_{13}=H_{31}=\frac{1}{4 E}\left(-\Delta m_{21}^{2} \sin ^{2} \theta_{12}+\Delta m_{31}^{2}\right) \sin 2 \theta_{13}, \\
& H_{22}=\frac{1}{2 E}\left(\Delta m_{21}^{2} \cos ^{2} \theta_{12}\right), \\
& H_{23}=H_{32}=-\frac{1}{4 E} \Delta m_{21}^{2} \sin 2 \theta_{12} \sin \theta_{13}, \\
& H_{33}=\frac{1}{2 E}\left(\Delta m_{21}^{2} \sin ^{2} \theta_{12} \sin ^{2} \theta_{13}+\Delta m_{31}^{2} \cos ^{2} \theta_{13}\right),
\end{aligned}
$$

where the common effective potential $V_{\mathrm{NC}}$ due to the neutron scatterers in matter 
has been left out, since it shifts the phases of all active neutrinos by the same amount.

With this Hamiltonian, the propagation equation for the system of three active neutrino states can be solved by using some of the numerical integration solvers. The default numerical solver for the first order differential equation at SNO is the Runge-Kutta $5^{\text {th }}$ order method (see for example Reference [94]). It is a very good method for solving differential equation when the properties of the medium change abruptly (which is the case for the interiors of the Sun and Earth). However, for the case of the three active neutrino states, this solver is found to be extremely inefficient. For example, the integration inside the Sun is performed by more that $10^{8}$ integration steps, which should be compared with the maximum number of steps used in the two-neutrino numerical computation of $10^{6}$. The routine that we found to be more efficient for the three neutrino case (by a factor of 10) is the Bulirsch-Stoer (BS) differential equation solver [100]. Thus, the recommend method to be used in the plausible SNO three-neutrino oscillation analysis is the BS method. 


\section{Appendix B}

\section{EML Method with Pulls}

The inclusion of correlated sources of errors in the extended maximum likelihood (EML) method is presented in this appendix.

The statistical part of the EML function is given in Equation (9.2). In order to perform solar neutrino oscillation analysis, it is necessary to include the systematic uncertainties associated with the data samples being tested. The method chosen to be implemented in our analysis with the EML function is the pull method, which is proposed, originally, for a case of the $\chi^{2}$ analysis in Reference [101].

In the pull method, the effect of each of the $K$ sources of systematic errors is included through the shift of the theoretical prediction for the observable $Y_{i}^{\text {th }}$ :

$$
Y_{i}^{\mathrm{th}} \rightarrow Y_{i}^{\mathrm{th}}+\sum_{k=1}^{K} \xi_{k} \Delta Y_{i}^{k}
$$

where $\xi_{k}$ is a random variable from normal (Gaussian) distribution, and $\Delta Y_{i}^{k}$ is a systematic error for the observable in bin $i$ induced by a source of systematic $k$. The shifted theoretical expectations for the observable $Y$ are used to construct 
a new likelihood function by adding the quadratic penalties for the $\xi_{k}$ random variable:

$$
-2 \ln L_{\text {pull }}=\min _{\left\{\xi_{k}\right\}}\left[-2 \ln L_{\text {shift }}+\sum_{k=1}^{K} \xi_{k}^{2}\right],
$$

where the function $-2 \ln L_{\text {shift }}$ is defined as:

$$
-2 \ln L_{\text {shift }}=2 \sum_{i=1}^{N}\left(Y_{i}^{\mathrm{th}}+\sum_{k=1}^{K} \xi_{k} \Delta Y_{i}^{k}\right)-2 \sum_{i=1}^{N} Y_{i}^{\exp } \ln \left(Y_{i}^{\mathrm{th}}+\sum_{k=1}^{K} \xi_{k} \Delta Y_{i}^{k}\right)
$$

SNO only pulls of systematic uncertainties

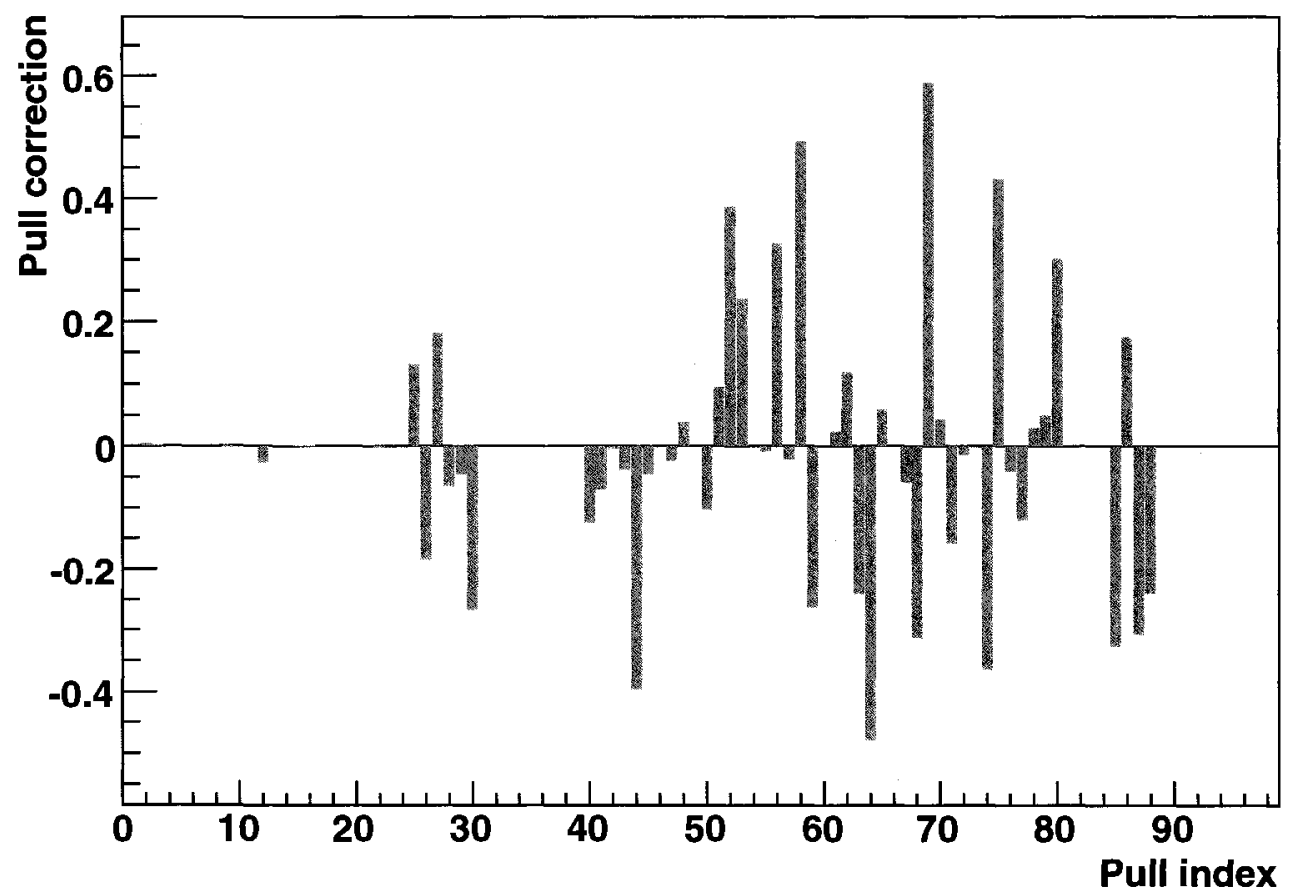

Figure B.1: Pulls of systematic uncertainties from the SNO-only EML analysis. The pulls are given in terms of fitted penalties $\xi_{k}$ (labelled as pull correction).

The function described in Equation (B.2) is minimized at each point of the 
parameter space (e.g. $\left.\Delta m^{2}, \tan ^{2} \theta\right)$ with respect to the quadratic penalties $\xi_{k}$. The values of the $\xi_{k}$ variables, where the EML function achieves its minimum, are defined as the "pulls" of systematic uncertainties, while the $-2 \ln L_{\text {shift }}$ part of the EML function defines the "pulls" of observables. The "pulls" of systematic uncertainties from the two-neutrino oscillation analysis of the data sets from the three phases of SNO are depicted in Figure B.1.

The EML function described here is used in the solar neutrino oscillation analysis presented in Chapter 9. 


\section{Glossary}

AMB The Analog Measurement Board.

AV The Acrylic Vessel.

CC The Charged-Current weak interaction.

CL The Confidence Level region.

CNO The Carbon-Nitrogen-Oxygen cycle of thermonuclear reactions in the Sun.

DQXX SNOMAN banks that hold electronic status from the PMT system for each run.

ES The Elastic Scattering of neutrinos on electrons.

FPS The First Pass Run Selection processor in SNOMAN.

GTID The General Time Identification number for an event at SNO.

GPS The Global Position System.

LEB The Low Energy Background at SNO.

LMA The Large Mixing Angle region in neutrino mixing parameter space. 
LOW The Low mass region in neutrino mixing parameter space.

LSP The Livetime Summary Processor in SNOMAN.

MC The Monte-Carlo method.

MCMC The Markov-Chain Monte Carlo method.

MSW The Mikheyev-Smirnov-Wolfenstein effect.

NC The Neutral-Current weak interaction.

NCBG The Neutral-Current Background at SNO.

NCD Neutral Current Detection array.

NGR NCD General Record events.

NHIT Number of Hits in PMT.

NP New Physics.

NSA+ Nakamura-Sato-Ando and others calculation of the neutrino-deuteron cross-section.

NQXX SNOMAN banks that hold electronic status from the NCD system for each run.

PGT Pulsed Global Trigger at SNO.

PMNS The Pontecorvo-Maki-Nakagawa-Sakata neutrino mixing matrix.

PMT Photomultiplier Tube. 
pp-chain Chain of thermonuclear reactions in the Sun that starts with the fusion of two protons.

RLDT The Run Logger Dead Time bank in SNOMAN.

SL The Sample Line assay at SNO.

SK The Super-Kamiokande experiment.

SM The Standard Model of particle physics.

SMA The Small Mixing Angle region in neutrino mixing parameter space.

SNOMAN SNO Monte-Carlo Analysis software.

SNO The Sudbury Neutrino Observatory.

SNP The Solar Neutrino Problem.

SNU Solar Neutrino Unit $\left(10^{-36}\right.$ captures per target atom per second).

SPS The Second Pass Run Selection processor in SNOMAN.

SSM The Standard Solar Model.

UC The Unusual Circumstances bit in the run header.

UTC The Universal Coordinated Time.

VAC The Vacuum region in neutrino mixing parameter space.

QVO The Quasi-Vacuum region in neutrino mixing parameter space. 


\section{Bibliography}

[1] W. Pauli. Letter to the Physical Society of Tübingen, Cambridge monogr. part. Phys. Nucl. Phys. Cosmol., 14, 1-22, 2000.

[2] E. Fermi. An Attempt of a Theory of Beta Radiation. 1, Z. Phys. 88, 161-177, 1934.

[3] E. Fermi. Trends to a Theory of Beta Radiation, Nuovo Cim. 11, 1-19 , 1934.

[4] C. Cowan et al. Detection of the Free Anti-Neutrino, Phys. Rev. 117, 159-173, 1960.

[5] S. Weinberg. A Model of Leptons, Phys. Rev. Lett. 19, 1264, 1967.

[6] A. Salam. Proc. of the $8^{\text {th }}$ Nobel Symposium on "Elementary Particle Physics, Relativistic Groups and Analyticity", Stockholm, Sweden, edited by N. Svartholm, p.367-377, 1968.

[7] M. Naki et al. Remarks on the Unified Model of Elementary Particles, Prog.Theor.Phys.28, 870, 1962.

[8] S. Fukuda et al. Evidence for Oscillation of Atmospheric Neutrinos, Phys.Rev.Lett. 81, 1562-1567, 1998. 
[9] B. T. Cleveland et al. Measurement of the Solar Electron Neutrino Flux with the Homestake Chlorine Detector, Astrophys. J. 496, 505-526, 1998.

[10] M. Altmann et al. (The GNO Collaboration). Complete Results for Five Years of GNO Solar Neutrino Observations, Phys. Lett. B 616, 174, 2005.

[11] J.N. Abdurashitov et al. (SAGE Collaboration)Measurement of the Solar Neutrino Capture Rate by the Russian-American Gallium Solar Neutrino Experiment During One Half of the 22-Year Cycle of Solar Activity, J. Exp. Theor. Phys. 95, 181-193, 2002; Zh. Eksp. Teor. Fiz. 122, 211-226, 2002.

[12] Y. Fukuda at al. Solar Neutrino Data Covering Solar Cycle 22, Phys. Rev. Lett. 77, 1683 - 1686, 1996.

[13] S. Fukuda et al. (The Super-Kamiokande Collaboration) Determination of Solar Neutrino Oscillation Parameters Using 1496 Days of SuperKamiokande-I Data, Phys. Lett. B539, 179-187, 2002.

[14] Q. R. Ahmad et al. (The SNO Collaboration) Direct Evidence for Neutrino Flavor Transformation from Neutral-Current Interactions in the Sudbury Neutrino Observatory, Phys. Rev. Lett. 89, 011301, 2002.

[15] E. Majorana. Theory of the Symmetry of Electrons and Positrons, Nuovo Cim.14, 171-184,1937.

[16] J. Lesgourgues and S. Pastor. Massive Neutrinos and Cosmology, Phys.Rep. 307-379, 2006.

[17] S. Hannestad. Primordial Neutrinos, arXiv:0602058 v1 [hep-ph], 2006. 
[18] R. N. Mohapatra et al. Theory of Neutrinos: A White Paper, Rept. Prog. Phys.70, 1757-1867, 2005.

[19] P. Ramond. The Family Group in Grand Unified Theories Invited talk at Sanibel Symphosium, 1979 arXiv:[hep-ph/9809459].

[20] M. Gell-Mann et al. in Supergravity, edited by P. van Nieuwenhuizen and D. Z. Freedman (North Holand) p. 315, 1979.

[21] T. Yanagida. Proceedings of Workshop on Unified Theory and Baryon Number in the Universe, 1979, edited by O. Sawada and A. Sugamoto (KEK report 79-18), 1979.

[22] The ALEPH, DELPHI, L3, OPAL, SLD Collaborations, the LEP Electroweak Working Group, the SLD Electroweak and Heavy Flavour Groups. Precision Electroweak Measurements on the $Z$ Resonance, Phys.Rept. 427, $257,2006$.

[23] S. M. Bilenky et al. Phenomenology of Neutrino Oscillations, Prog. Part. Nucl. Phys. 43, 1-86, 1999.

[24] S. Eliezer et al. Experimental Consequences of $\nu_{e}-\nu_{\mu}$ Mixing in Neutrino Beams, Nuc. Phys. B 105, 45-51, 1976.

[25] H. Fritzsch et al. Vectorlike Weak Currents, Massive Neutrinos, and Neutrino Beam Oscillations, Phys. Let. B 62, 72, 1976.

[26] S. M. Bilenky et al. Lepton Mixing and Neutrino Oscillations, Sov. Phys. Usp. 20 No 10, 776-795, 1977. 
[27] C. Giunti et al. Fundamentals of Neutrino Physics and Astrophysics, Oxford University Press, 2007 [ISBN 978-0-19-850871-7].

[28] B. Pontecorvo, Sov. Phys. JETP, 6, 429, 1957.

[29] M. Apollonio et al. (The CHOOZ Collaboration) Search for Neutrino Oscillations on a Long Base-line at the CHOOZ Nuclear Power Station, Eur. Phys. J. C27, 331-374, 2003.

[30] P.C. de Holanda and A. Yu. Smirnov. Homestake Result, Sterile Neutrinos and Low Energy Solar Neutrino Experiments, Phys. Rev. D 69, 113002, 2004.

[31] T. K. Kuo and J. Pantaleone. Neutrino Oscillation in Matter, Rev. Mod. Phys., 61, 937-978, 1989.

[32] L. Wolfenstein. Neutrino Oscillations in Matter, Phys. Rev. D 17, 2369 $2374,1978$.

[33] S.P. Mikheev and A.Yu. Smirnov. Resonance Amplification of Oscillation in Matter and Spectroscopy of Solar Neutrinos, Sov. J. Nucl. Phys.42, 913917,1985 (Yad. Fiz. 42, 1441-1448,1985).

[34] S.P. Mikheev and A.Yu. Smirnov. Resonance Amplification of Oscillation in Matter and Spectroscopy of Solar Neutrinos, Nuovo Cim.C9, 17-26,1986.

[35] H. A. Bethe. Energy Production in Stars Phys. Rev. 55, 434-456, 1939.

[36] M. Fukuda and T. Yanagida. Physics of Neutrinos, Springer-Verlag, New York, 2003, [ISBN 3-540-43800-9]. 
[37] J. N. Bahcall et al. Standard Solar Models and the Uncertainties in Predicted Capture Rates of Solar Neutrinos, Rev. Mod. Phys. 44, 767-799, 1982.

[38] J. N. Bahcall et al. Solar Model: Current Epoch and Time Dependences, Neutrinos and Helioseismological Properties, Astroph. Journ., 555:900-1012, 2001.

[39] J. N. Bahcall et al. What Do We (Not) Know Theoretically about Solar Neutrino Fluxes, Phys. Rev. Lett., 92, 121301, 2004.

[40] J. N. Bahcall et al. New Solar Opacities, Abundances, Helioseismology and Solar Neutrino Fluxes Astrophys. Journ., 621, L83, 2005.

[41] R. J. Davis et al. Search for Neutrinos from the Sun, Phys. Rev. Lett. 20, $1205-1209,1968$.

[42] J. N. Bachall et al. Present Status of the Theoretical Predictions for the ${ }^{37} \mathrm{Cl}$ Solar-Neutrino Experiment*, Phys. Rev. Lett. 20, 1209 - 1212, 1968.

[43] J. N. Bahcall, URL:http://www.sns.ias.edu/ jnb/.

[44] J.N. Abdurashitov et al. (SAGE Collaboration), Neutrino 2004 Conference, (Paris, June 2004).

[45] B. Aharmim et al. (The SNO Collaboration). A Search for Neutrinos from the Solar hep Reaction and the Diffuse Supernova Neutrino Background with the Sudbury Neutrino Observatory, ApJ 653, 1545, 2006.

[46] J.P. Cravens et al. (The SK Collaboration). Solar neutrino measurements in Super-Kamiokande-II arXiv:0803.4312v1 [hep-ex], 2008. 
[47] C. Arpesellaa et al. (The Borexino Collaboration) New Results on Solar Neutrino Fluxes from 192 Days of Borexino Data, arXiv:0805.3843v2 [astro-ph], 2008.

[48] B. Aharmim et al.(The SNO Collaboration) Electron Energy Spectra, Fluxes, and Day-Night Asymmetries od ${ }^{8} B$ Solar Neutrinos from the 391-Day Salt Phase SNO Data Set, Phys. Rev. C72, 055502, 2005.

[49] J. Boger et al. (The SNO Collaboration). The Sudbury Neutrino Observatory Nuc. Instrum. Meth. A449, 172-207, 2000.

[50] P. Vogel et al. Angular Distribution of Neutron Inverse Beta Decay, $\bar{\nu}_{e}+p \rightarrow$ $e^{+}+n$, Phys. Rev. D. 60, 053003, 1999.

[51] B. Aharmim et al. (The SNO Collaboration). An Independent Measurement of the Total Active ${ }^{8}$ B Solar Neutrino Flux Using an Array of 3He Proportional Counters at the Sudbury Neutrino Observatory, Phys. Rev. Lett. 101, 111301, 2008.

[52] M. Bouley. Direct Evidence for Weak Flavour Mixing with the Sudbury Neutrino Observatory, Ph. D. Thesis, Queen's University, 2001.

[53] F. Zhang. Electron Energy Response of NCD Phase Dataset, SNO technical report, 2008.

[54] J. Orell. The $50 \mathrm{MHz}$ Clock Document, SNO internal document, 2000.

[55] K. Schaffer Miknaitis. A Search for Matter Enhanced Neutrino Oscillation through Measurements of Day and Night Solar Neutrino Fluxes at the Sudbury Neutrino Observatory, Ph. D. Thesis, University of Washington, 2005. 
[56] N. McCauley. Producing a Background Free Data Set for Measurements of the Charged Current Flux and Day-Night Asymmetry at the Sudbury Neutrino Observatory, Ph. D. Thesis, University of Oxford, 2001.

[57] J. Detwiler, N. McCauley, K. Rielage, G. Tešić and F. Zeng. Livetime for the Full NCD Data Set,SNO Internal Report, 2008.

[58] SNOMAN Companion 5.03, SNO Internal Document.

[59] The SNO Collaboration, URL:http://www.sno.phy.queensu.ca/sno/ prlwebpage/.

[60] N. Metropolis et al. Equation of State Calculations by Fast Computing Machines, Journ. Chem. Phys. 21, 1087, 1953.

[61] W.K. Hastings. Monte Carlo Sampling Methods Using Markov Chains and Their Applications, Biometrika 57, 97, 1970.

[62] B. Jamieson. SNO NCD Phase Signal Extraction on Unblinded Data with Integration over Systematic Nuisance Parameters by Markov-Chain MonteCarlo, the SNO internal document, 2008.

[63] W.T.Winter et al. The ${ }^{8}$ B Neutrino Spectrum, Phys. Rev. C73, 025503, 2006.

[64] Q. R. Ahmad et al. (The SNO Collaboration). Measurement of Day and Night Neutrino Energy Spectra at SNO and Constraints on Neutrino Mixing Parameters, Phys. Rev. Lett. volume 89, No. 1, 011302, 2002.

[65] B. Aharmim et al. (The SNO Collaboration), Measurement of the $\nu_{e}$ and Total ${ }^{8}$ B Solar Neutrino Fluxes with the Sudbury Neutrino Observatory Phase I Data Set, Phys. Rev. C75, 045502, 2007. 
[66] J. Wilson. A Measurement of the ${ }^{8} B$ Solar Neutrino Energy Spectrum at the Sudbury Neutrino Observatory, Ph. D. Thesis, University of Oxford, 2004.

[67] K. Graham. Salt Data Reconstruction Analysis, SNO technical report, 2004.

[68] B. Beltran et al. NCD MC Readiness Report for the First NCD Paper, SNO technical report, 2008.

[69] The SNO Collaboration, URL: http://owl.phy.queensu.ca/private/qphysics/, SNO physics interpretation web page.

[70] A. Bellerive. Combination and Comparison of Measurements from Different Phases of SNO, SNO technical report, 2008.

[71] J. Secrest. Energy Systematics for Phase III, SNO technical report, 2008.

[72] J. Hosaka et al. (The Super-Kamiokande Collaboration) Solar Neutrino Measurements in Super-Kamiokande-I, Phys. Rev. D73, 112001, 2006.

[73] S. N. Ahmed et al. (The SNO Collaboration) Measurement of the Total Active ${ }^{8} B$ Solar Neutrino Flux at the Sudbury Neutrino Observatory with Enhanced Neutral Current Sensitivity, Phys. Rev. Lett. 92, 181301, 2004.

[74] M. Chen and Y. Takeuchi. QPhysics Updates, SNO technical report, 2003.

[75] A. J. Baltz et al. Effect of Transmission through the Earth on Neutrino Oscillations, Phys. Rev. D 35, 528 - 535, 1987.

[76] A. J. Baltz et al. Matter oscillations: Neutrino transformation in the Sun and regeneration in the Earth, Phys. Rev. D 37, 3364 - 3377, 1988. 
[77] C. Arpesellaa et al. (The Borexino Collaboration) First Real Time Detection of ${ }^{7}$ Be Solar Neutrinos by BorexinoAuthors, Phys.Lett. B658, 101-108, 2008.

[78] S. Nakamura et al. Neutrino-Deuteron Reactions at Solar Neutrino Energies, Nucl.Phys. A707, 561-576, 2002.

[79] G. t Hooft. Prediction for Neutrino-Electron Cross-Sections in Weinberg's Model for Weak Interactions, Phys. Lett. 37B, 195, 1971.

[80] J.N. Bahcall et al. Solar Neutrinos: Radiative Corrections in NeutrinoElectron Scattering Experiments, Phys. Rev. D 51 6146, 1995.

[81] H. Robertson. Long $\mathrm{D}_{2} \mathrm{O}$ Paper: Target Properties, SNO technical report, 2007.

[82] A. Bellerive. How-to Use the SNO NCD Flux Results, SNO technical report, 2008, URL: http://www.sno.phy.queensu.ca/sno/papers/ncd_chi2/.

[83] G. Cowan. Statistical Data Analysis, Oxford University Press, 1998.

[84] S. Nakamura et al. Neutrino-Deuteron Reactions at Solar Neutrino Energies, Nucl. Phys. A707, 561-576, 2002.

[85] C. E. Ortiz et al. Shape of the ${ }^{8} B$ Alpha and Neutrino Spectra, Phys. Rev. Lett. 85, $2909-2912,2000$.

[86] J. N. Bahcall et al. Standard Neutrino Spectrum from ${ }^{8} B$ Decay, Phys.Rev. C54, 411-422, 1996.

[87] G. L. Fogli et al. Standard solar model uncertainties and their correlations in the analysis of the solar neutrino problem, Astropart. Phys. 3, 185, 1995. 
[88] J. N. Bahcall et al. How do Uncertainties in the Surface Chemical Abundances of the Sun Affect the Predicted Solar Neutrino Fluxes?, Astrophys. Journ. $626,530,2005$.

[89] T. Araki et al. Measurement of Neutrino Oscillation with KamLAND: Evidence of Spectral Distortion, Phys. Rev. Lett. 94, 081801, 2005.

[90] S. Abe et al. Precision Measurement of Neutrino Oscillation Parameters with KamLAND, Phys. Rev. Lett. 100, 221803, 2008.

[91] P.C. de Holanda and A. Yu. Smirnov. Private Communication.

[92] J.S.Kim and K.Lee. The Earth Regeneration of Solar Neutrinos: a Numerical Treatment with Three Active Neutrino Flavors, Comp. Phys. Comm. 135, 176-189, 2001.

[93] R. Martin. Numerical Methods and Approximations for the Calculation of Solar Neutrino Fluxes in Three Flavours Focused on Results for the Sudbury Neutrino Observatory, Masters Thesis, Queens University, 2006.

[94] W. H. Press et al. Numerical Recipes in C++: The Art of Scientific Computing, Cambridge University Press, 2007.

[95] G. Bellini et al. (The Borexino Collaboration). Measurement of the Solar ${ }^{8} B$ Neutrino Flux with 246 Live Days of Borexino and Observation of the MSW Vacuum-Matter Transition, arXiv:0808.2868v1 [astro-ph], 2008.

[96] P. Adamson et al. (The Minos Collaboration). Measurement of Neutrino Oscillations with the MINOS Detectors in the NuMI Beam, FERMILAB-PUB08-168-E, arXiv:0806.2237v1 [hep-ex], 2008. 
[97] S. Goswami and A. Yu. Smirnov. Solar Neutrinos and 1-3 Leptonic Mixing, Phys.Rev. D72, 053011, 2005.

[98] G. L. Fogli et al. Global Analysis of Three-Flavor Neutrino Masses and Mixings, Prog. Part. Nucl. Phys. 57, 742-795, 2006.

[99] G. L. Fogli et al. Hints of $\theta_{13}>0$ from Global Neutrino Data Analysis, arXiv:0806.2649v1 [hep-ph], 2008.

[100] J. Stoer and R. Bulirsch. Introduction to Numerical Analysis, SpringerVerlag, New York, 1980.

[101] G. L. Fogli et al. Getting the Most from the Statistical Analysis of Solar Neutrino Oscillations, Phys. Rev. D66, 053010, 2002. 